\title{
MASS-SPECTROMETRY BASED METABOLOMICS TO DECIPHER STRAIN SPECIFIC MICROCYSTIS CYANOPEPTIDE PROFILES
}

by

\author{
Kimberlynn Pearl McDonald
}

A thesis submitted to the Faculty of Graduate and Postdoctoral Affairs in partial fulfillment of the requirements for the degree of

Master of Science

in

Chemistry with Specialization in Chemical and Environmental Toxicology

Carleton University

Ottawa, Ontario

(C) 2020, Kimberlynn Pearl McDonald 


\section{i. Abstract}

Over the last one hundred years, ecosystem changes have occurred as a result of human population growth, pollution, increased temperatures, and habitat degradation. A visible change is the increase in frequency and magnitude of toxic cyanobacterial blooms. Cyanobacterial blooms release mixtures of biologically active compounds into freshwater that negatively impact human and ecosystem health as well as having socioeconomic consequences. The factors that influence cyanobacterial growth and toxin production are broadly understood. However, cyanobacteria are a prolific source of structurally diverse and strain specific mixtures of biologically active compounds. Currently, the chemistry, toxicology, environmental concentrations, and risks posed to human and ecosystem health by most cyanobacterial secondary metabolites are unknown. Advances in mass spectrometry and metabolomic techniques can aid in comprehension of complex metabolomes. Here, the use of untargeted and semi-targeted mass spectrometry-based metabolomics is used to decipher the non-ribosomal peptide natural products (cyanopeptides) from five Microcystis strains. Cyanopeptides are grouped based on shared structural features, such as the incorporation of non-proteogenic amino acids or partial amino acid sequences that generate diagnostic product ions with the MS/MS of metabolites within the same cyanopeptide group. Global natural product society (GNPS) molecular networking and diagnostic fragmentation filtering (DFF) techniques utilize the similarity in product ion spectra to visualize all variants in the different cyanopeptide groups and the production by Microcystis strains. The semi-targeted DFF technique is applied to environmental water and bloom samples for the detection of entire cyanopeptide groups. Finally, growth medium composition effects on Microcystis chemical profiles are assessed with the established metabolomic techniques. Optimal growth conditions for cyanopeptide production have been elucidated for the Microcystis strains. This will enable the 
growth of target strains that produce novel compounds and common environmental contaminants on a large scale for the isolation and purification of new cyanopeptides. With tangible material of novel cyanopeptides, structural and toxicological assessments as well as quantification of environmental concentrations can be performed. 


\section{ii. Acknowledgements}

I would first like to thank my supervisor, David McMullin, for providing the opportunity to develop and expand the cyanopeptide project with interdisciplinary work. Thank you to Justin Renaud for his expertise with advanced mass-spectrometry and metabolomics as well as Dr Mark Sumarah for the experience in the LRDC lab to better expand our analytical techniques. And thanks to Natasha Desrochers for her metabolomic talents and quick replies. I am grateful for the opportunity to attend the Gordon Research Conference on Phycotoxins and Mycotoxins which expanded my research interests and provided inspiration to my own work.

Thank you to Dr David Miller for many projects and experiences over the course of my time at Carleton University that helped me build up my skills and critical thought processes as a scientist.

Thank you to Francis Pick for the collection and detailed information for the bloom samples collected in the National Capital Region analyzed here.

Thank you to the fellow graduate students for the support and encouragement throughout my time at Carleton University, from brainstorming experimental approaches to trivia night at Mike's Place. I would also like to thank the dedicated undergraduate students that helped perform experiments and did the grunt work.

Finally, thank you to my parents and sisters for the never-ending love and support through this experience. 


\section{iii. Table of Contents}

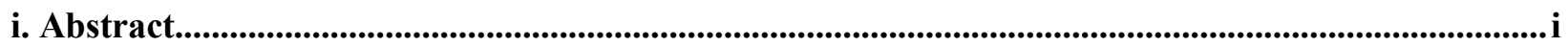

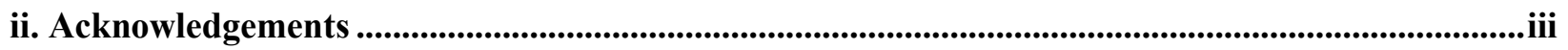

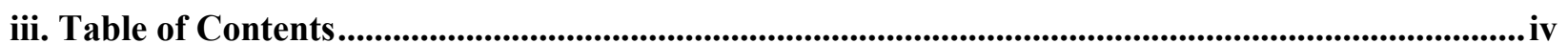

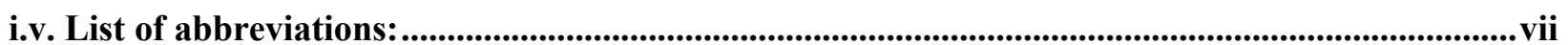

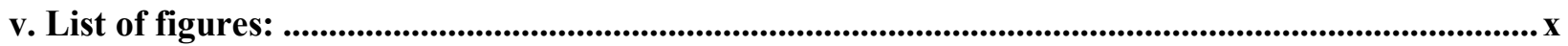

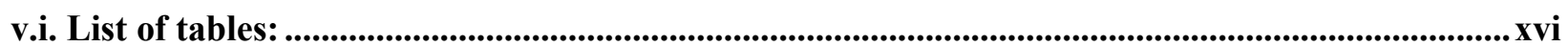

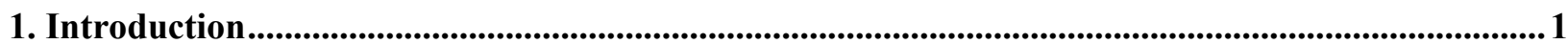

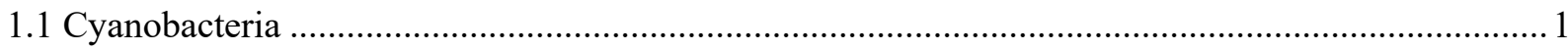

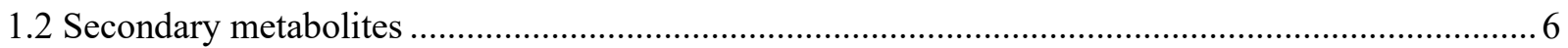

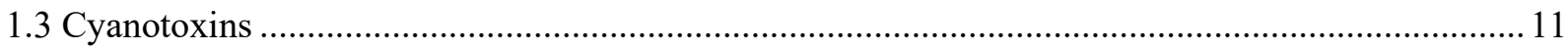

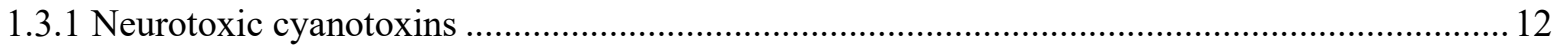

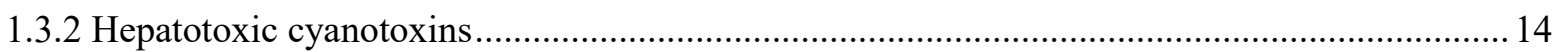

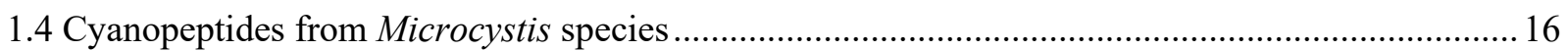

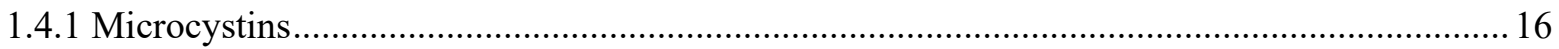

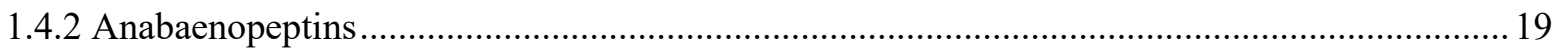

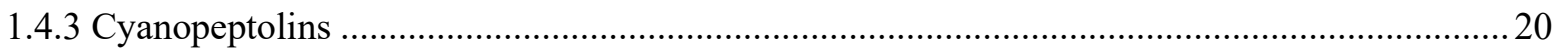

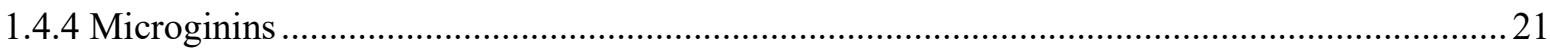

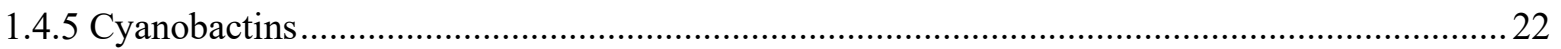

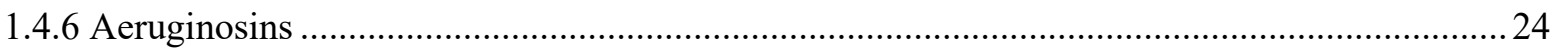

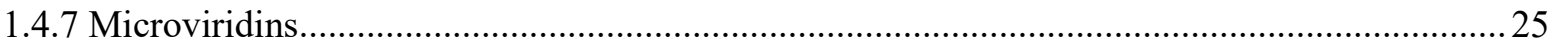

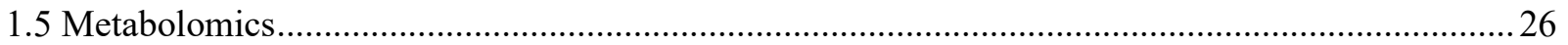

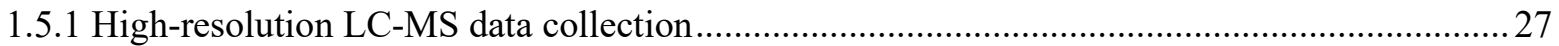

1.5.2 Untargeted mass-spectrometry based metabolomics ...............................................................29

1.5.3 Semi-targeted mass-spectrometry based metabolomics.......................................................... 31

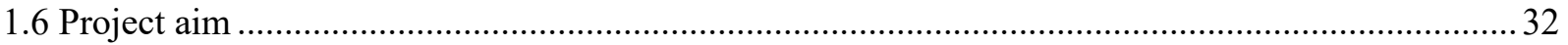

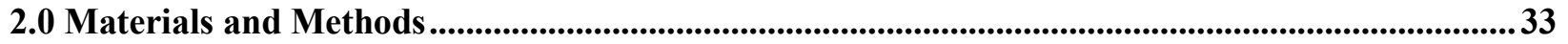

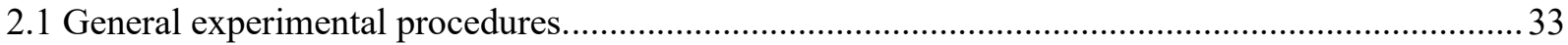

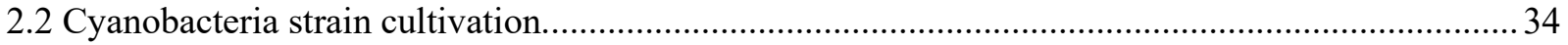

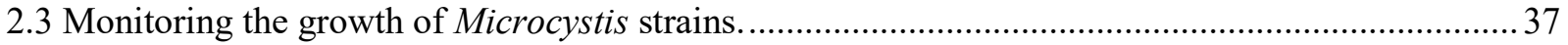

2.4 Extraction of cyanopeptides from Microcystis cells..................................................................... 37

2.5 Cyanopeptide extraction from environmental samples................................................................. 38

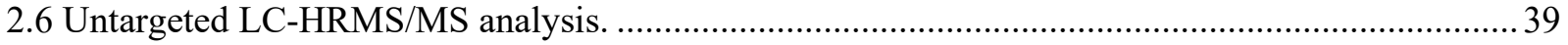




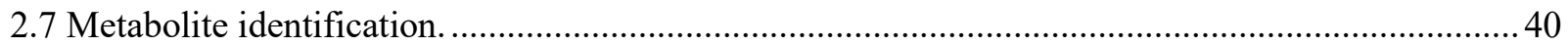

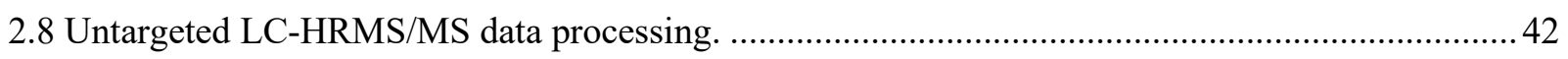

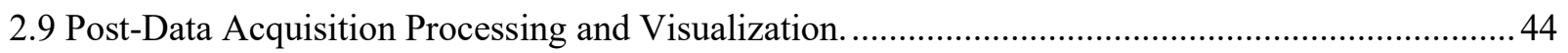

2.9.1 Principal component analysis and factor loading analysis.................................................... 44

2.9.2 Heat map analysis to depict media effects on cyanopeptide production................................... 45

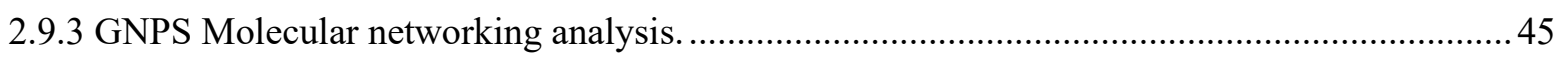

2.9.4 Diagnostic fragmentation filtering (DFF) for cyanopeptide group detection. ......................... 46

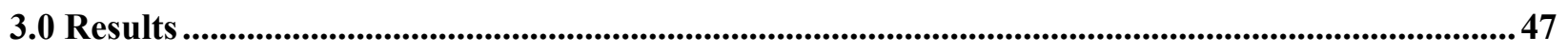

3.1 Untargeted mass-spectrometry based metabolomics: application to cyanopeptide groups ..............47

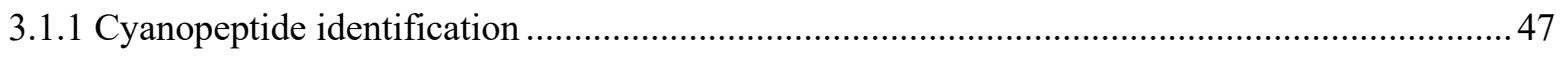

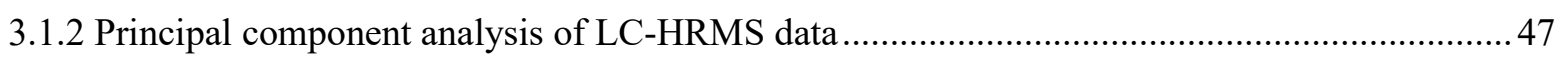

3.1.3 Molecular network analysis of LC-HRMS data.................................................................. 54

3.1.4 Relative abundance of cyanopeptides produced by Microcystis strains .................................. 82

3.2 Semi-targeted mass-spectrometry based metabolomics: application to cyanopeptide groups...........84

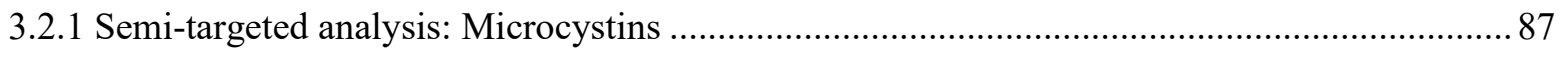

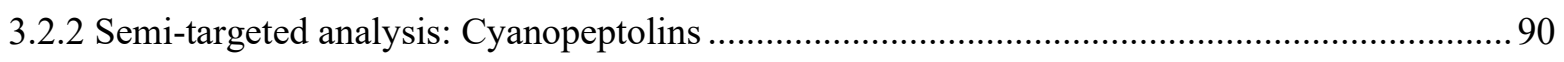

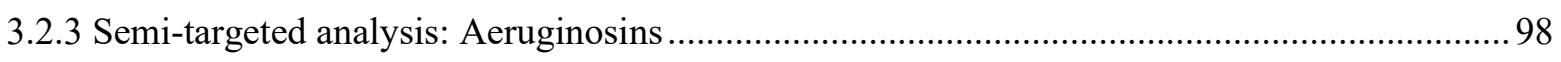

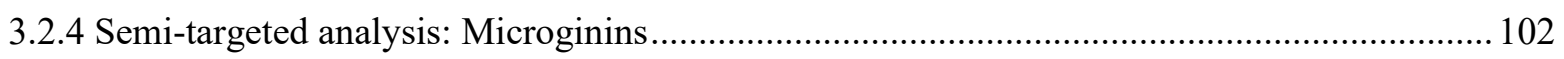

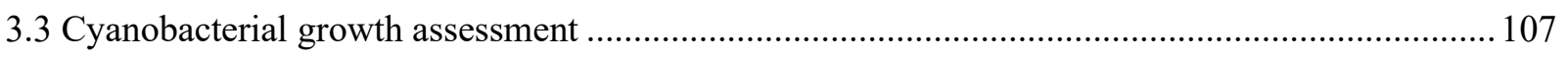

3.4 Investigation of cyanopeptide production by Microcystis strains.............................................. 111

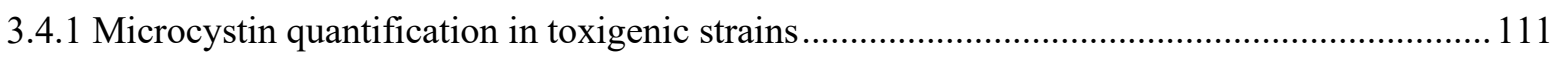

3.4.2 Effects of media composition on the production of cyanopeptides by Microcystis strains ..... 114

3.4.2a Media effects on cyanopeptide production by M. aeruginosa CPCC 300 ........................... 114

3.4.2b Media effects on cyanopeptide production by $M$. flos-aquae CPCC 461 .............................. 117

3.4.2c Media effects on cyanopeptide production by M. aeruginosa CPCC 632 ............................ 119

3.4.2d Media effects on cyanopeptide production by M. aeruginosa CPCC 633 ........................... 122

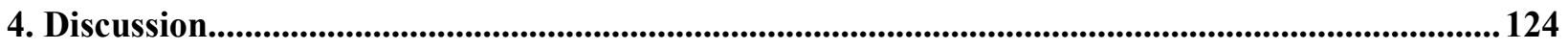

4.1 Untargeted mass-spectrometry based metabolomics to decipher strain specific variation between

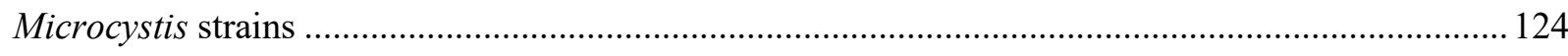

4.2 Semi-targeted mass-spectrometry based metabolomics for detection of cyanopeptide groups in

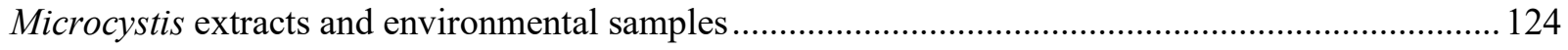

4.3 Media composition effects on cyanopeptide production of Microcystis strains ........................... 132

4.4 Discussion of the toxicity of the five Microcystis strains ........................................................... 139

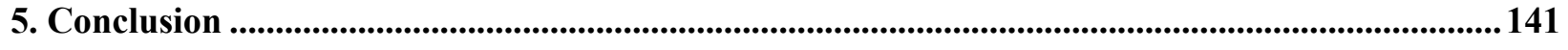

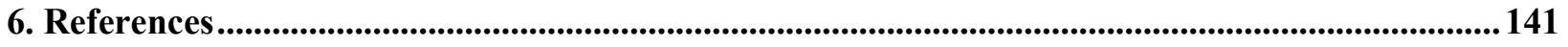


7. Supplementary Information 


\section{i.v. List of abbreviations:}

A/Ala - alanine

ACN - acetonitrile

Adda - 3-amino-9-methoxy-10-phenyl-2,6,8-trimethyldeca-4,6-dienoic acid

Ahda - 3- amino-2-hydroxy decanoic acid

Ahoa - 3-amino-2-hydroxy octanoic acid

Ahp - 3-amino-6-hydroxy-2-piperidone

ANOVA - analysis of variance

ATX - anatoxin

BW - body weight

$\mathrm{C} / \mathrm{Cys}$ - cysteine

Choi - 2-carboxy-6-hydroxyoctahydroindole

CPCC - Canadian Phycological Culture Collection

DCM - dichloromethane

$\mathrm{ddH}_{2} \mathrm{O}$ - distilled deionized water

DFF - diagnostic fragmentation filtering plot

DmA - 1,1-dimethylallyl

E/Glu - glutamic acid

F/Phe - phenylalanine

G/Gly-glycine

GNPS - global natural products society

$\mathrm{H} / \mathrm{His}$ - histidine

Hph - homo-phenylalanine

Hpla - hydroxy-phenyllactic acid

Hty - homo-tyrosine

HxA - hexanoic acid

I/Ile - isoleucine

$\mathrm{K} /$ Lys - lysine

KW - Kruskal Wallis test 
L/Leu - leucine

LC - liquid chromatography

$\mathrm{M} / \mathrm{Met}$ - methionine

$m / z$ - mass- to- charge ratio

$\mathrm{MC}$ - microcystin

Mdha - methyl-dehydroalanine

Mdhb - 2-(methylamino)-2-dibutyric acid

$\mathrm{MeOH}$ - methanol

MS - mass spectrometry

$\mathrm{MS} / \mathrm{MS}$ - tandem mass spectrometry

$\mathrm{N} / \mathrm{O}-\mathrm{Me}-\mathrm{N}-/ \mathrm{O}-\mathrm{methylated}$

NOD - nodularin

nRP - non-ribosomal peptide

nRPS - non-ribosomal peptide synthase

OATPs - organic anion transporting polypeptides

P/Pro - proline

PCA - principal component analysis

PKS - polyketide synthase

$\mathrm{PP} 1 / 2 \mathrm{~A}$ - protein phosphatase $1 / 2 \mathrm{~A}$

Q/Gln - glutamine

QTofF - quadrupole time-of-flight

$\mathrm{R} / \mathrm{Arg}$ - arginine

RiPP - post-translationally modified peptide

S/Ser - serine

sp. - species

spp. - multiple species

STX - saxitoxin

$\mathrm{T} / \mathrm{Thr}$ - threonine 
Thz - thiazole

Tzc - thiazole carboxylate

Tzl - thiazoline

V/Val - valine

W/Trp - tryptophan

Y/Tyr - tyrosine 


\section{v. List of figures:}

Figure 1.1: Researchers investigating the Microcystis harmful algal bloom in Lake Erie in August 2019 (Johnson, Cleverland.com).

Figure 1.2: Experimental lake 226 divided with a curtain with half fertilized with phosphorous to show the impact phosphorous has on bloom formation.

Figure 1.3: Simplified mechanism of non-ribosomal peptide synthesis. (1) The amino acid is activated by the adenylation domain. (2) Transfer of the amino acid onto the PCP domain. (3) Condensation of PCP-bound amino acids. (4) Possibility of amino acid modifications, for example by epimerization domains. (5) Transesterification of the peptide chain from the terminal PCP onto the TE domain. (6) TE domain catalyzed product release by either hydrolysis, aminolysis, or macrocyclization. The number of modification domains and modules is highly variable (Strieker et al., 2010).

Figure 1.3: Chemical structure of neurotoxin cyanotoxins, anatoxin-a (left), saxitoxin (centre), and BMAA (right).

Figure 1.4: Chemical structure of cylindrospermopsin.

Figure 1.5: Chemical structure of hepatotoxic cyanopeptides nodularin-R (left) and microcystinLR (right).

Figure 1.6: Chemical structure of the microcystin congener, MC-LR. Adda; 3-amino-9-methoxy10-phenyl-2,6,8-trimethyldeca-4,6-dienoic acid, Mdha; methyl-dihydroalanine, Mdhb; 2(methylamino)-2-dibutyric acid.

Figure 1.7: Structure of the anabaenopeptin congener produced by Microcystis sp., Ferintoic acid A.

Figure 1.8: Chemical structure of cyanopeptolin A (left) and cyanopeptolin 954 (right). Ahp; 3amino-6-hydroxy-2-piperidone, HxA; hexanoic acid, Ac; acetyl.

Figure 1.9: Chemical structure of microginin 680 (left) and microginin KR 604 (right). Ahoa; 3amino-2-hydroxy octanoic acid, NMeAhda; 3-NMe-amino-2-hydroxy decanoic acid.

Figure 1.10: Chemical structure of aerucyclamide A (left) and microcyclamide A (right). Tzl; thiazole, Tzn; thiazoline, $M e O z l$; methyl-oxazole, $M e O z n$; methyl-oxazoline.

Figure 1.11: Chemical structure of aeruginosamide B (left) and C (right).

Figure 1.12: Chemical structure of aeruginosin 98A. Choi; 2-carboxy-6-hydroxyoctahydroindole, Hpla; hydroxy-phenyllactic acid.

Figure 1.13: Representative schematic showing the scaffold of microviridin cyanopeptides. Chemical structure of microviridin $\mathrm{N}$ is shown.

Figure 2.1: Microcystis strains growing in $250 \mathrm{~mL}$ Erlenmeyer flasks in a growth chamber.

Figure 2.2: Workflow of the unique LC-MS/MS metabolomic data processing performed. 
Figure 3.1: Principal component analysis (PCA) of metabolites produced by triplicate cultures of Microcystis aeruginosa CPCC 299, 300, 632, and 633, and M. flos-aquae CPCC 461 grown in BG-11 medium.

Figure 3.2: The loading plot contains all factor loadings considered. Of 787 total variables, 262 are considered in the PCA and visualized in the loading plot. Factors included fall within the precursor ion range $m / z 400-1200$ (unless present within the molecular network) to target cyanopeptides, 2.0-6.6 min retention time range, and above a peak threshold of $5 \mathrm{E}^{+06}$. Red arrows indicate statistical significance in contribution to the individual strains in the PCA $(p<0.05$ Kruskal-Wallis test with Benjamin-Hochberg correction). Specific cyanotoxins identified by their precursor ion $m / z$, MS/MS data and retention time are numbered.

Figure 3.3: GNPS molecular network generated from metabolite MS/MS spectra produced by the five studied Microcystis strains grown in BBM medium. Nodes are color coded pi-charts labelled with precursor ion $\mathrm{m} / \mathrm{z}$ illustrating which strains produced each metabolite (qualitative). The width of lines connecting nodes are proportional to the corresponding cosine score. Precursor and product ion $\mathrm{m} / \mathrm{z}$ tolerance were set to 0.03 and $0.02 \mathrm{Da}$, respectively. The minimum base peak intensity was set at $5 \mathrm{E}^{+06}$. Clustering parameters required a minimum cosine similarity score of 0.6 and five matching product ions. Deconvolution was performed by eliminating all metabolites present within the blank, outside the retention time range 2-6.5 minutes, and clusters with a size less than 2. Cyanotoxin groups and specific metabolites identified using the GNPS spectral library, interpretation of MS/MS spectra and comparisons to the literature are labelled. Abbreviations used in this figure include Ac; aerucyclamide, Ag; aeruginosamide, Ahp; 3-amino-6-hydroxy-2piperidone, AP; anabaenopeptin, Cyp; cyanopeptolin, Lxx; leucine or isoleucine, MC; microcystin, Md; microcyclamide, $\mathrm{Mg}$; microginins and $\mathrm{Mv}$; microviridin.

Figure 3.4: The microcystin GNPS network cluster 6 and LC-MS analysis of the congeners produced by M. aeruginosa CPCC 299 (red) and CPCC 300 (yellow). The structure if MC-LR is showed with labeled residues (top right). The MS/MS of MC-LR (bottom left) and [Asp ${ }^{3}$, Dha ${ }^{7}$ MC-LR (bottom right) with diagnostic product ions and their putative structures, $m / z \quad 135.0803$ and 163.1113 of the Adda moiety, and $\mathrm{m} / \mathrm{z} 213.0865$ of the Mdha.

Figure 3.5: The two cyanopeptolin GNPS clusters, Phe-Ahp cyanopepetolins, cluster 1 (top left) and Lxx-Ahp cyanopeptolins, cluster 2 (bottom left) and representative MS/MS spectra for congeners within these subclasses. Chemical structures of Cyanopeptolin 954 (top right) and Cyanopeptolin A (bottom right) are two examples of each class. Diagnostic product ions for each class are generated and resulted in two cyanopeptolin clusters. Key product ions to differentiate the subclasses are highlighted on the spectra. For Phe-Ahp cyanopeptolins, $m / z$ 184.0517 (NMeCl-Tyr), 215.1167 ([Ahp-Phe-CO- $\left.\mathrm{H}_{2} \mathrm{O}\right]^{+}$) are present and for Lxx-Ahp cyanopeptolins $\mathrm{m} / \mathrm{z}$ 134.0961 (NMePhe), 181.1331 ([Ahp-Lxx-CO- $\left.\left.\mathrm{H}_{2} \mathrm{O}\right]^{+}\right)$.

Figure 3.6: LC-MS analysis (middle) and anabaenopeptin GNPS cluster 9 (top left) show that $M$. aeruginosa CPCC 632 (grey) and CPCC 633 (blue) produce the same anabaenopeptins (AP). Representative MS/MS spectra are shown for ferintoic acid A (bottom left) and new structure, AP 850 (bottom right). The structure of ferintoic acid A (top right) was determined by its $[\mathrm{M}+\mathrm{H}]^{+}$ion at $m / z 867.4378[\mathrm{M}+\mathrm{H}]^{+}$and immonium ions corresponding to each residue in its chemical 
structure: $m / z$ 58.0658 (NMe-Ala), $m / z 72.0792$ (Val), $m / z$ 84.0812 (Lys), $m / z 120.0835$ (Phe), $m / z$ $150.0910(\mathrm{Hph})$ and $\mathrm{m} / z 159.0914$ (Trp). The molecular formula of anabaenopeptin 850 suggests a loss of an oxygen atom compared to ferintoic acid A. The MS/MS spectrum of anabaenopeptin 850 showed a product ion at $\mathrm{m} / z 134.0964$ (Hph) instead of a Hty immonium ion accounting for the structural change.

Figure 3.7: The microginin GNPS cluster (7) shows that M. aeruginosa CPCC 632 (grey) and CPCC 633 (blue) produce different mixtures of microginins (Mg). LC-MS analysis (top right) of co-eluting Mg 612 (Ahoa), 646 (Cl-Ahoa) and $680\left(\mathrm{Cl}_{2}\right.$-Ahoa) differ by a $\mathrm{Cl}$ atom. The structure of Mg 612 (top left) was determined by its MS/MS spectrum (bottom left) that showed product ions at $m / z 100.1122$ (Ahoa), $m / z 70.0658$ (Pro), $m / z 136.0752$ (Tyr) and $m / z 150.0910$ (NMeTyr). The structure of a related new variant, $\mathrm{Mg}$ 660, with a rare $\mathrm{NMe}-\mathrm{Cl}$-Ahoa moiety was identified by a product ion at $m / z 148.0833$ (Cl-NMe-Ahoa, bottom right).

Figure 3.8: A microviridin GNPS network cluster (4, top left) and LC-MS analysis (top right) of congeners produced by M. aeruginosa CPCC 299, CPCC 632, and CPCC 633. Full MS scan at 2.76 minutes (bottom left) shows the precursor ion for Microviridin $\mathrm{J}\left(\mathrm{m} / z\right.$ 842.8925 $\left.[\mathrm{M}+2 \mathrm{H}]^{2+}\right)$ which was identified by precursor ion $\mathrm{m} / \mathrm{z}$ and product ions within the MS/MS (bottom right) of $m / z 70.0659$ (Pro), 84.0808 (Lys), 136.0729 (Tyr), and 159.0935 (Trp).

Figure 3.9: The aeruginosin GNPS clusters 10 (grey, $M$. aeruginosa CPCC 632) and 11 (yellow, $M$. aeruginosa CPCC 300) from the total GNPS network and the congener information. MS/MS of As 688 (bottom left) and As 650 (bottom right) with diagnostic product ions $m / z 140.1069$ and 122.0966 of the Choi functionality. Further grouping of this chemical class is done with Phe $(\mathrm{m} / \mathrm{z}$ 120.0807) or Tyr ( $\mathrm{m} / \mathrm{z}$ 136.0756) substitutions in position 2 , as well as the possibility of sulfated Choi $\left(\left[\mathrm{M}+\mathrm{H}-\mathrm{SO}_{3}\right]^{+}\right)$. Partial amino acid sequence was concluded with the product ion corresponding to [Hpla-Residue $2-\mathrm{CO}]^{+}$which for those in cluster 10 and containing a Phe in position 2 have $m / z 284.1268$ and for those in cluster 11 containing a Tyr in position 2 have $\mathrm{m} / \mathrm{z}$ 300.1232 .

Figure 3.10: Aerucyclamide GNPS network cluster (13, top left) with LC-MS analysis of the congeners produced by M. aeruginosa CPCC 299 (red), 300 (yellow), and 461 (green). Identity of aerucyclamide A (top right) was confirmed by precursor ion $\mathrm{m} / \mathrm{z}$ and diagnostic product ions from

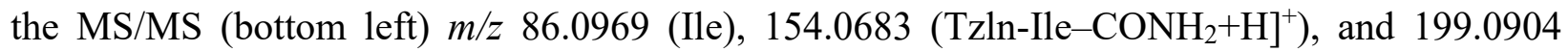
(Tzln-Ile). Potential new aerucyclamide 518 was confirmed as reduced aerucyclamide $\mathrm{C}$ with diagnostic ions aligning with Tzln functionality $\mathrm{m} / \mathrm{z} 154.0683$ and 199.0905 , as well as $\mathrm{m} / \mathrm{z}$ 167.0838 of the Val-Ozl product ion not present in aerucyclamide A or B.

Figure 3.11: Microcyclamide GNPS network cluster (12, top left) with LC-MS analysis of the congeners produced by M. aeruginosa CPCC 299 (red) and M. flos-aquae 461 (green). Identity of microcyclamide A was confirmed with precursor ions $m / z 261.0798$ (Ala-Tzl-MeHis) 213.0645 (MeHis-Tzl), 153.0656 (MeOxazole-Ala), and 96.0685 (MeHis). Potential new microcyclamides 584 and 586 contain the oxidized Tzl (Tzln), in position 5 for Md 584 and in position 3 and 5 for Md 586.

Figure 3.12: The aeruginosamide (linear cyanobactin) GNPS cluster (8, top left) and the congener information. The structure of aeruginosamide B is shown (top right) and the MS/MS spectra of 
aeruginosamide B (bottom left) and aeruginosamide 560 (bottom right) is shown with diagnostic product ions labeled and putative structures shown. Other immonium ions to prove the identity of aeruginosamide B are $m / z 120.0807$ (Phe), 70.0658 (Pro), and 144.0109 (Tzc).

Figure 3.13: The GNPS network cluster (14, top right) for the unknown chemical class with similar congeners produced by CPCC 299 (red) and CPCC 633 (blue). LC-MS analysis of the congeners (top right) with an MS spectrum at 5.65 minutes to show coelution of two congeners, and the MS/MS of the congener Unk $1490\left(\mathrm{~m} / z 746.4204[\mathrm{M}+2 \mathrm{H}]^{2+}\right)$ with proteogenic immonium ions $\mathrm{m} / z 70.0659$ (Pro), $102.0553(\mathrm{Gln}), 136.0756$ (Tyr), and the characteristic product ion $\mathrm{m} / \mathrm{z}$ 115.0865 .

Figure 3.14: Relative abundance of metabolites on day 15 when grown in $\mathrm{BG}-11$ media at $27^{\circ} \mathrm{C}$, generated with normalized peak area of identified secondary metabolites produced by 4 Microcystis aeruginosa strains CPCC 299 (top left), CPCC 300 (middle left), CPCC 632 (bottom left), CPCC 633 (middle right) and 1 Microcystis flos-aquae strain CPCC 461 (bottom right). Metabolites coloured based on cyanopeptide group.

Figure 3.15: MS/MS spectrum of MC-LR $\left(\mathrm{m} / z\right.$ $\left.995.5565[\mathrm{M}+\mathrm{H}]^{+}\right)$with representative structures of product ions generated from the Adda moiety, $\mathrm{m} / z 135.0803$ and $\mathrm{m} / \mathrm{z} 163.1113$.

Figure 3.16: Diagnostic fragmentation filter plot targeting Adda-containing metabolites produced by M. aeruginosa CPCC 300 searching for the product ions $\mathrm{m} / z$ 135.0803 and $\mathrm{m} / \mathrm{z} 163.1113$, characteristic of the Adda moiety.

Figure 3.17: Representative diagnostic fragmentation filtering plot targeting Adda-containing metabolites within the environmental sample \#3 searching for the product ions $\mathrm{m} / \mathrm{z} 135.0803$ and $\mathrm{m} / \mathrm{z} 163.1113$, characteristic of the Adda moiety.

Figure 3.18: $37.5 \mathrm{NCE}$ MS/MS spectra of cyanopeptolin A (left) and nostopeptin BN920 (right) produced by M. aeruginosa CPCC 300 and CPCC 632, respectively. MS/MS spectra for each cyanopeptolin are displayed when cyanobacteria were grown in BG-11 (top) and $\mathrm{Na}^{15} \mathrm{NO}_{3}$ supplemented BG-11 (bottom) media. (Top left) Diagnostic product ions for cyanopeptolin A at $\mathrm{m} / z 134.0963$ and 181.1331 correspond to $\mathrm{NMePhe}$ at position 2 and Leu-Ahp at positions 3 and 4 , respectively. (Bottom left) Incorporation of ${ }^{15} \mathrm{~N}$ into peptide scaffolds supports these diagnostic product ions. Corresponding NMePhe and Leu-Ahp product ions are observed at $m / z 135.0935$ and 183.1281. (Top right) Diagnostic product ions for nostopeptin BN920 at $\mathrm{m} / \mathrm{z} 150.0912$ and 215.1183 correspond to NMeTyr at position 2, and Leu-Ahp at positions 3 and 4, respectively. (Bottom right) Incorporation of ${ }^{15} \mathrm{~N}$ into peptide scaffolds supports these product ions observed at 151.0883 and 217.1119 , that contain 1 and 2 nitrogen atoms, respectively.

Figure 3.19: Representative DFF plots for cyanopeptolins containing NMe-Phe-Lxx produced by M. aeruginosa CPCC 300 (top) and NMe-Tyr-Phe containing cyanopeptolins produced by $M$. aeruginosa CPCC 632 (bottom). Red horizontal lines are indicative of diagnostic product ions required to be considered cyanopeptolins with designated residues at position 2 and 3 . The black solid line represents $[\mathrm{M}+\mathrm{H}]^{+}$or $\left[\mathrm{M}+\mathrm{H}-\mathrm{H}_{2} \mathrm{O}\right]^{+}$precursor ions as designated in Table 2. Each vertical dot represents product ions detected within the MS/MS spectra of corresponding cyanopeptolin precursor ions. 
Figure 3.20: Representative cyanopeptolin DFF plots for water samples 3 (left) and 4 (right). DFF plots for cyanopeptolins containing the partial sequence NMe-Tyr-Lxx-Ahp (left) and the partial sequence Cl-NMe-Tyr-Phe-Ahp (right).

Figure 3.21: MS/MS spectra of aeruginosin 688 produced by $M$. aeruginosa CPCC 300. MS/MS spectra for the aeruginosin congener are displayed when cyanobacteria were grown in BG-11 (left) and $\mathrm{Na}^{15} \mathrm{NO}_{3}$ supplemented BG-11 (right) media. (Top left) Diagnostic product ions for aeruginosin 688 at $m / z 140.1099$ and $m / z 122.0964$ correspond to the [Choi $+\mathrm{H}]^{+}$and [Choi $-\mathrm{H}_{2} \mathrm{O}$ $+\mathrm{H}]^{+}$, respectively. Incorporation of ${ }^{15} \mathrm{~N}$ into peptide scaffolds supports these diagnostic product ions. Corresponding $[\mathrm{Choi}+\mathrm{H}]^{+}$and $\left[\mathrm{Choi}-\mathrm{H}_{2} \mathrm{O}+\mathrm{H}\right]^{+}$product ions are observed at $\mathrm{m} / z$ 141.1029 and $\mathrm{m} / \mathrm{z} 123.0936$ that contain 1 nitrogen atom each.

Figure 3.22: Representative diagnostic fragmentation filter plot searching for Choi containing metabolites found in bloom samples 5 and 8, with the product ion $m / z 140.1066\left([\mathrm{Choi}+\mathrm{H}]^{+}\right)$and $122.0964\left(\left[\mathrm{Choi}-\mathrm{H}_{2} \mathrm{O}+\mathrm{H}\right]^{+}\right)$.

Figure 3.23: MS/MS spectra of microginin 646 produced by $M$. aeruginosa CPCC 632. MS/MS spectra for microginin 646 are displayed when cyanobacteria were grown in BG-11 (left) and $\mathrm{Na}^{15} \mathrm{NO}_{3}$ supplemented BG-11 (right) media. (Top left) Diagnostic product ions for microginin 646 at $\mathrm{m} / z 150.0910$ and $\mathrm{m} / z 134.0727$ correspond to the NMeTyr and Cl-Ahoa, respectively. Incorporation of ${ }^{15} \mathrm{~N}$ into peptide scaffolds supports these diagnostic product ions. Corresponding $\mathrm{NMeTyr}$ and Cl-Ahoa product ions are observed at $\mathrm{m} / z 151.0869$ and $\mathrm{m} / z 135.0684$ that contain 1 nitrogen atom each.

Figure 3.24: Diagnostic fragmentation filter plot of microginins produced by $M$. aeruginosa CPCC 633 when searching for the different product ions that correlate to the N-terminal amino acid. Colours are used to display the variable chain length (Ahoa, NMeAhoa, Ahda, NMeAhda) and shape to designate the chlorination state (non-, mono-, di-).

Figure 3.25: Representative diagnostic fragmentation filter plots of microginins detected within environmental samples 4 (left) and 8 (right) when searching for the different product ions that correlate to the N-terminal amino acid. Colours are used to display variable chain length and substitution (Ahoa, NMeAhoa, Ahda, NMeAhda) and shape to designate the chlorination state (non-, mono-, di-).

Figure 3.26: Growth data for M. aeruginosa CPCC 300 (top left), M. flos-aquae CPCC 461 (top right), M. aeruginosa CPCC 632 (bottom left), and M. aeruginosa CPCC 633 (bottom right) depicting growth curve measurements including cells $\mathrm{mL}^{-1}$ and dried biomass (mg). Red lines indicate measurements when strains grown in MA media and blue lines indicate measurements when strains grown in BG-11 media. Squares indicate cells $\mathrm{mL}^{-1}$ and circles indicate biomass in mg.

Figure 3.27: $\mathrm{pH}$ and optical density at $750 \mathrm{~nm}$ for $M$. aeruginosa CPCC 300 (top left), M. flosaquae CPCC 461 (top right), M. aeruginosa CPCC 632 (bottom left), and M. aeruginosa CPCC 633 (bottom right) grown in BG-11 (orange) and MA (blue) growth media. pH data points are represented by circles and optical density data points are represented with squares.

Figure 3.28: Quantification of intracellular MC-LR ( $\mu$ g MC-LR mg biomass ${ }^{-1}$ ) production by $M$. aeruginosa CPCC 300 when grown in the two culture media BG-11 (orange) and MA (blue). 
Figure 3.29: Heatmap illustrating cyanopeptide production by M. aeruginosa CPCC 300 over time in two growth media (BG-11;left and MA;right), generated with the average normalized peak area ( $\log _{10}$, top). Tukey's LSD test distinguished groups designated with letters to illustrate quantitative differences in the individual cyanotoxin production profile (statistically significant, $p<0.05$ ). Data points with the same letter within a cyanotoxin column are not statistically significant from one another. Cell count measurements are depicted to correlate toxin production with the phase of growth (bottom).

Figure 3.30: Heatmap depicting cyanotoxin production by $M$. flos-aquae CPCC 461 over time in two growth media (BG-11;left and MA;right), generated with the average normalized peak area ( $\log _{10}$, top). Tukey's LSD test distinguished groups designated with letters to illustrate quantitative differences in the individual cyanotoxin production profile (statistically significant, $p<0.05$ ). Data points with the same letter within a cyanotoxin column are not statistically significant from one another. Cell count measurements are depicted to correlate toxin production with the phase of growth (bottom).

Figure 3.31: Heatmap depicting cyanotoxin production by $M$. aeruginosa CPCC 632 over time in two growth media (BG-11;left and MA;right), generated with the average normalized peak area $\left(\log _{10}\right.$, top). Tukey's LSD test distinguished groups designated with letters to illustrate quantitative differences in the individual cyanotoxin production profile (statistically significant, $\mathrm{p}<0.05$ ). Data points with the same letter within a cyanotoxin column are not statistically significant from one another. Cell count measurements are depicted to correlate toxin production with the phase of growth (bottom).

Figure 3.32: Heatmap depicting cyanotoxin production by M. aeruginosa CPCC 633 over time in two growth media (BG-11;left and MA;right), generated with the average normalized peak area $\left(\log _{10}\right.$, top). Tukey's LSD test distinguished groups designated with letters to illustrate quantitative differences in the individual cyanotoxin production profile (statistically significant, $\mathrm{p}<0.05$ ). Data points with the same letter within a cyanotoxin column are not statistically significant from one another. Cell count measurements are depicted to correlate toxin production with the phase of growth (bottom). 


\section{v.i. List of tables:}

Table 2.1: Cyanobacteria strain information from the Canadian Phycological Culture Collection.

Table 2.2: Media components for the defined culture media used to grow Microcystis species.

Table 2.3: Collection information for bloom and water samples.

Table 2.4: Unique structural features characteristic of different cyanopeptide groups.

Table 2.5: The peak picking parameters used with the bioinformatics package, $x \mathrm{cms}$.

Table 2.6: Common adducts generated by electrospray ionization in positive mode.

Table 3.1: Summary of all cyanopeptolins detected within the GNPS molecular network.

Table 3.2: Collection information for Microcystis strains and environmental samples.

Table 3.3: Product ions specific to the Adda moiety present within metabolites of the MC chemical group.

Table 3.4: Microcystins detected by DFF within Microcystis strains and environmental samples.

Table 3.5: Product ions specific to the partial amino acid sequences present within metabolites of the cyanopeptolin cyanopeptide group.

Table 3.6: Cyanopeptolins detected by DFF from Microcystis aeruginosa strains and environmental samples.

Table 3.7: Product ions specific to the Choi moiety present within metabolites of the aeruginosin cyanopeptide group.

Table 3.8: Chemical information of the Choi-containing metabolites produced by $M$. aeruginosa CPCC 300 and CPCC 632 and found within environmental water and bloom samples detected by DFF.

Table 3.9: Product ions specific to the variable N-terminal polyketide moiety present within metabolites of the microginin cyanopeptide group.

Table 3.10: Chemical information of the microginins produced by M. aeruginosa CPCC 632 and CPCC 633 and found in surface water and bloom samples.

Table 3.11: Strain information and specific growth rates $\left(\right.$ day $\left.^{-1}, \mu\right)$ for four Microcystis strains grown at $27^{\circ} \mathrm{C}$ in different culture media.

Table 3.12: Summary of LC-HRMS data for eight MC congeners and NOD with reference material.

Table 3.13: Intracellular MC-LR levels for M. aeruginosa CPCC 300 grown in BBM media at $27^{\circ} \mathrm{C}$.

Table 3.14: Maximum relative abundance for selected metabolites produced by $M$. aeruginosa CPCC 300 when grown in two culture media with an ANOVA pairwise comparison $(p<0.05)$ to add significance to the media effects. 
Table 3.15: Maximum relative abundance for selected metabolites produced by $M$. flos-aquae CPCC 461 when grown in two culture media with an ANOVA pairwise comparison $(p<0.05)$ to add significance to the media effects.

Table 3.16: Maximum relative abundance for selected metabolites produced by $M$. aeruginosa CPCC 632 when grown in two culture media with an ANOVA pairwise comparison $(p<0.05)$ to add significance to the media effects.

Table 3.17: Maximum relative abundance for selected metabolites produced by $M$. aeruginosa CPCC 633 when grown in two culture media with an ANOVA pairwise comparison $(p<0.05)$ to add significance to the media effects.

Table S1: LC-HRMS data for cyanotoxins produced by M. aeruginosa CPCC 299, 300, 632 and 633, and M. flos-aquae CPCC 461.

Table S2: Compiled sequence database for cyanopeptolins previously reported in literature.

Table S3: Compiled sequence database for microginins previously reported in literature. 


\section{Introduction}

\section{$\underline{1.1 \text { Cyanobacteria }}$}

Cyanobacteria or "blue-green algae" are ubiquitous photosynthetic prokaryotes that inhabit aquatic ecosystems including freshwater lakes, marine coastal areas, and drinking water reserves. The long evolutionary history of cyanobacteria has provided them with adaptations to thrive in diverse environmental conditions (Paerl \& Millie, 1996). Under normal conditions cyanobacteria will remain at low cell numbers within the environment and not pose a serious threat to ecosystem or human health. The factors that influence cyanobacteria growth are broadly understood and include sunlight, access to macronutrients including nitrogen and phosphorous, and water temperature (Walls et al., 2018). With increased nutrient input from anthropogenic sources as well as an increase in surface water temperatures due to climate change, the excessive proliferation and accumulation of cyanobacterial colonies results in visible surface blooms (cyanobacterial harmful algal blooms, cHABs, Figure 1.1) which can have adverse outcomes. The decomposition of dead cyanobacterial biomass results in decreased dissolved oxygen amounts within the water column. The production of stagnant zones by dense algal mats can generate strong vertical dissolved oxygen gradients and ultimately, anoxic conditions which have been linked with fish mortalities (Watson et al., 2015). Further, the release of cyanobacterial toxins into water during cell senescence pose poorly characterized risks to ecosystem and human health. Further, toxin producing blooms have socioeconomic consequences as a result of contaminated drinking water, irrigation reservoirs, and the diminishment of recreational value in freshwater lakes (Paerl \& Otten, 2013). 


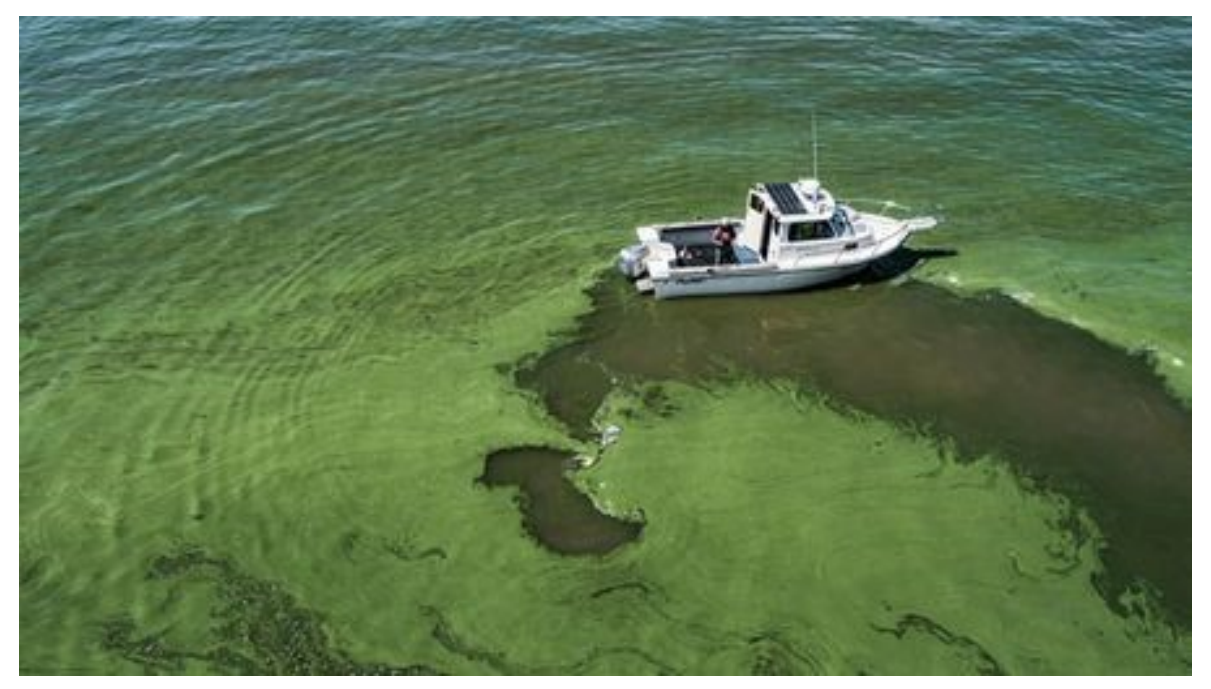

Figure 1.1: Researchers investigating the Microcystis harmful algal bloom in Lake Erie in August 2019 (Johnson, Cleveland.com).

With excess nutrients, the main determinant of cyanobacterial growth and metabolism is temperature because many enzymes that participate in photosynthesis and respiration are temperature dependent (Walls et al., 2018). Cyanobacteria typically dominate the freshwater algae populations at higher temperatures due to their optimum growth rates at such temperatures and their increased affinity for nutrients at higher temperatures in comparison to eukaryotic algae (Figure 1.1). The determinants of surface accumulation and thus bloom formations are highly variable and are dependent on water depth as well as short term changes in weather such as solar radiation and windspeed. Shallow waters paired with low precipitation and high spring temperatures can result in more intense bloom formation (Pick, 2016). 
When temperature is constant, the essential nutrients for cyanobacterial growth can be determined.

A famous study performed in the 1970s used an entire lake to show the importance of nutrients on cyanobacteria bloom formation and found phosphorous to be the nutrient with the largest impact (Schindler \& Fee, 1974). The lake was divided in two with a membrane. One half of the lake was fertilized with phosphorous, nitrogen, and carbon $(+\mathrm{P})$ and the other half fertilized with only nitrogen and carbon (-P, Figure 1.2). The half of the lake fertilized with phosphorous developed a bloom whereas the other side did not.

$-\mathrm{P}$

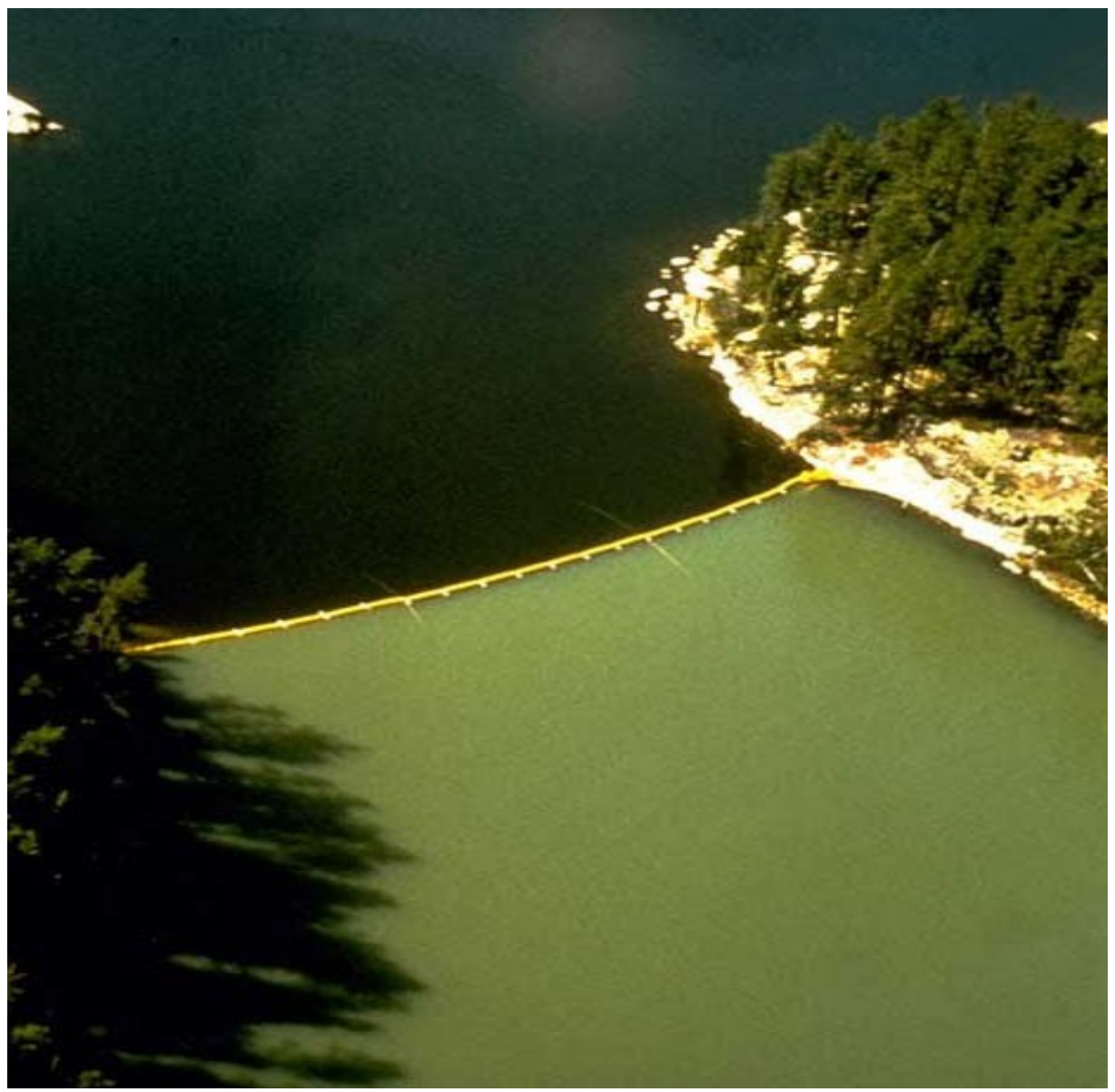

Figure 1.2: Experimental lake 226 divided with a curtain with half fertilized with phosphorous to show the impact phosphorous has on bloom formation.

Cyanobacteria have several evolutionary adaptations that have provided them with competitive advantages over microalgae species. The competitive advantages include efficiently acquiring 
nutrients, buoyancy control allowing an overwintering process, and cyanotoxin production. When water temperatures drop below a level that is optimal for cyanobacterial growth, the cells stock up on their photosynthetically synthesized sugars, sink to benthic layers, slow down primary metabolism and halt secondary metabolism to survive the colder water temperatures (Henao et al., 2020). In the spring, an event known as spring turn over occurs releasing oxygen from the water and results in turn over of the sediment, releasing the cyanobacteria. This is the inoculum for the next years bloom season. This buoyancy control is achieved with the generation of vacuoles which contain photosynthetic oxygen and enable cyanobacteria to access nutrients suspended along the vertical water column (Paerl \& Millie, 1996). Another competitive advantage is toxin production (cyanotoxin) that occurs in common bloom forming cyanobacteria genera such as Microcystis, Nodularia, Anabaena, Aphanizomenon, and Dolichospermum. Cyanotoxins have diverse structures and are grouped at a high level based on their modes of action which can include with hepatotoxins, neurotoxins, dermatoxins and enzyme inhibitors (Huang \& Zimba, 2019). Another competitive advantage that has evolved for some filamentous cyanobacteria genera such as Anabaena, Nostoc, and Cylindrospermopsis are heterocysts, specialized nitrogen-fixing cells that metabolize $\mathrm{N}_{2}$ gas and nitrates from the atmosphere and water (Paerl \& Otten, 2013). Within heterocytes, $\mathrm{N}_{2}$ gas and nitrates are reduced to amines which are subsequently exported and used by photosynthetic cells and incorporated into complex biomolecules (Zurawell et al., 2005).

Cyanobacteria growth rates vary based on the species, or even strains of the same species, with variable optimal temperatures and nutrient concentrations. Toxic strains of cyanobacteria require larger quantities of nitrogen and phosphorous due to the expense of energy and materials for toxin biosynthesis (Zurawell et al., 2005). Non-heterocystous cyanobacteria such as Microcystis and Planktothrix require higher nitrogen concentrations for increased toxicity whereas heterocystous 
cyanobacteria show increased toxicity when grown in a nitrogen free growth medium. This indicates that toxin production varies in response to ambient nitrogen concentration for these two types of cyanobacteria (Rapala et al., 1997). Further, phosphorous concentrations are linked with toxin production for both heterocystous and non-heterocystous cyanobacteria. There appears to be a negative correlation with toxin production and phosphorous concentration, suggesting phosphorous limitation is a main factor that induces toxin production (Zurawell et al., 2005).

Toxin production increases at warmer temperatures due to increased photosynthetic rates and it is suggested that warmer temperatures impact the proportion of intracellular to extracellular toxin concentrations (Walls et al., 2018). Typically, the toxins remain cell-bound while cyanobacteria cells are alive. Upon cell death and lysis, intracellular cyanopeptides are released into the environment. There are very few defined mechanisms for extracellular toxin release (Walls et al., 2018). Research has shown that the release of secondary metabolites can prevent cellular damage when high photosynthetic output is coupled with unfavourable growth conditions (Smith \& Underwood, 2000).

Cyanobacterial strains from the genus Microcystis are the focus of this investigation. This genus represents one of the most prolific bloom-forming cyanobacteria with reports of blooms containing Microcystis species in over 108 countries with 79 countries reporting association of the hepatotoxins, microcystins, to the bloom (Harke et al., 2016). Microcystis occurrence within freshwater phytoplankton communities has increased drastically in the last 15 years, with previous reports of Microcystis sp. in less than 30 countries (Zurawell et al., 2005). Microcystis species are non-heterocystous, meaning they cannot fix $\mathrm{N}_{2}$ and thus must have other cellular advantages over heterocystous cyanobacteria. The physiological characteristics of Microcystis sp. that favour their ubiquitous dominance over other cyanobacteria species are their optimal growth occurs at $27^{\circ} \mathrm{C}$ 
and ability to produce complex mixtures of toxic secondary metabolites (cyanotoxins) which provides tolerance to zooplankton grazing (Le Manach et al., 2019). In 2014, the Toledo municipal water supply was contaminated by a Microcystis bloom that produced large quantities of microcystins, a group of potent hepatotoxins. 400,000 people were left without drinking water for several days while restoration and decontamination efforts were set in place. Large quantities of aluminum and chlorine were added to the water to coagulate the bloom so that biomaterial could be filtered from the water. However, extracellular release of the toxin could not be controlled with this approach. In 2014, there were no US National standards for safe cyanobacteria or cyanobacterial toxin levels within drinking and recreational water which resulted a demand for guidelines to be investigated/released (McKindles et al., 2020).

\section{$\underline{1.2 \text { Secondary metabolites }}$}

Living organisms complete a series of anabolic and catabolic reactions catalyzed by enzymes in a process known as metabolism to synthesize and degrade organic and inorganic materials (A. L. Demain \& Fang, 2000). Primary metabolism provides biosynthetic intermediates and energy that are converted into essential macromolecules such a DNA, RNA, proteins, lipids, and polysaccharides. Primary metabolism is a highly regulated and balanced process with the most important resulting molecules (primary metabolites) being nucleic acids, purine nucleotides, vitamins, and organic acids. Primary metabolism is relatively conserved across all living systems as it aids in growth, development, and reproduction (A. L. Demain \& Fang, 2000). Secondary metabolism however, primarily occurs in plants and microorganisms, and is usually strain-specific. Secondary metabolites are defined as small biomolecules that are not necessary for growth and development. The compounds have alternative functions that act to improve the survival of the producing organism in competition with other living species. Products of secondary metabolism 
are of interest to human health as approximately $25 \%$ of all natural products are bioactive and have helped double the human life span during the $20^{\text {th }}$ century through drug development. Roughly $75 \%$ of all antibiotics have been obtained from filamentous prokaryotes (Arnold L. Demain, 2014). Secondary metabolites are classified by the route of synthesis into the following categories: alkaloids, polyketide, terpenes, and non-ribosomal peptides. Bioactivities from the reported 23,000 natural products can range from antiviral, cytotoxic, anticancer, and immunosuppressive providing an excellent source for drug candidates (A. L. Demain \& Fang, 2000).

Cyanobacteria are known to produce a wide array of bioactive metabolites such as terpenoids, polyketides, depsipeptides, and peptides (Portmann, Blom, Gademann, et al., 2008). The function of these metabolites is proposed as a defensive agent against predators, as many purified cyanotoxins have shown toxicity against grazer crustaceans. Many reports indicate that these metabolites inhibit protease enzymes suggesting an alternative function once within the predator, preventing the degradation of the toxin before reaching its target (Janssen, 2019). Secondary metabolite production may also aid in reducing oxidative stress by diverting energy from the light harvesting complexes. It has been shown that secondary metabolites are often processed, cycled, and disposed of during regular cell growth but may accumulate intra- or extra-cellularly in significant quantities upon environmental stressors. However, most environmental signals that regulate secondary metabolism in cyanobacteria have yet to be deciphered. Overall, it is hypothesized that the production of such large and complex metabolites provides a control mechanism to overcome growth rate disadvantages among microbes when environmental conditions do not provide a competitive growth advantage (Paerl \& Millie, 1996). This link to bacterial growth rate further suggests coupling of toxin production to photosynthesis (Walls et al., 2018). The synthesis of these large biomolecules is an energic process and requires large amounts 
of energy, thus when the cells are rapidly dividing and performing photosynthesis at an optimal rate, excess energy is available for secondary metabolite production.

More than 2000 cyanobacteria natural products have been described to date. Oligopeptides (cyanopeptides) derived from non-ribosomal peptide synthetase (NRPS), mixed NRPS-polyketide synthase (NRPS-PKS), and post-translationally modified peptide (RiPP) pathways account for most of this reported chemical diversity (Jones et al., 2020). Other common biosynthetic pathways found in cyanobacteria generate alkaloid or terpene secondary metabolites. Cyanopeptides are the main focus in this investigation and are biosynthesized through via several non-ribosomal and ribosomal pathways such as non-ribosomal peptide synthase (NRPS) and polyketide synthase (PKS) enzyme systems or they may be synthesized via ribosomally synthesized and posttranslationally modified peptide (RiPP) pathways (Tiam et al., 2019). A modular structure of NRPSs is responsible for the sequential amino acid specific extension of peptide chains. The various combinations of modules and their functional domains determines the structure of the final peptide and the associated biological activity (Neilan, Dittmann, Rouhiainen, Bass, Schaub, Sivonen, \& Bo, 1999). This mode of synthesis for NRPS can incorporate unusual and nonproteinaceous amino acids into the sequence. As well, some of the modules may be polyketide synthases and result in the addition of polyketide molecular features within a peptide natural product. An example of this combined synthesis is that of congeners in the microginin chemical class (Figure 1.9). These metabolites have proteinaceous amino acids and an $\mathrm{N}$-terminal decanoic acid derivative biosynthesized by a polyketide synthase.

The modular sections within NRPSs have distinct functions responsible for the addition of a defined monomer into one complete natural product (Strieker et al., 2010). This assembly line defines which monomeric units are incorporated, in which particular order, the length and 
functionality of the metabolite, as well as the chemical reaction that occurs to link the monomers. There are 4 catalytic steps in NRP synthesis: activation, propagation, condensation, and release (Figure 1.3). The first step, activation, is catalyzed by an adenylation domain (A domain) and must be highly selective and specific toward the incoming monomer. The A domain acts as a gatekeeper enzyme for incoming amino acids and has a 10 amino acid specificity-conferring code which is responsible for substrate binding within the active site and ultimately determines the structure of the final NRP. Sequencing of the A domain and online specificity prediction tools are available and can aid in the isolation of predicted NRPS products (Strieker et al., 2010). Each A domain has a large $\mathrm{N}$-terminal core domain and a small C-terminal subdomain which are connected by a 5-10 residue hinge. The terminal domain amino acid sequences are highly conserved throughout all adenylating enzymes, including acetyl-coA synthase (Strieker et al., 2010). With the help of an ATP molecule, $\mathrm{Mg}^{2+}$ as a cofactor, and the specific monomeric amino acid, the first reaction in NRP synthesis occurs and the activated amino acid is transferred to the peptidyl carrier protein (PCP) also known as the thiolation domain, as it contains the prosthetic group 4'phosphopantetheine (Süssmuth \& Mainz, 2017). The now tethered amino acid is moved towards the condensation domain ( $\mathrm{C}$ domain) where it undergoes a condensation reaction with the nascent peptide. While the activated amino acid is tethered to the PCP, it is possible to be passed to accessory enzymes before reaching the $\mathrm{C}$ domain. These accessory enzymes have individual catalytic functions that allow modifications such as methylation, epimerization, heterocyclization, formylation, oxidation and reduction of the amino acid prior to incorporation which aids in increased structural diversity of cyanopeptides (Süssmuth \& Mainz, 2017). The finished product is released from the distal end of the assembly line by the termination domain (Te domain) through either hydrolysis, aminolysis, or macrocyclization liberating linear or cyclic products. The Te 
domain may cyclize molecules by aiding in folding the linear peptide and catalyzing an intramolecular nucleophilic attack from one end of the metabolite to another (Figure 1.3, Tillett et al., 2000).

Increased structural diversity of cyanopeptides occurs via decreased specificity of the enzymes present within the biosynthetic assembly line for amino acids. Although majority of NRPS have a high specificity for one single amino acid as a substrate, it is possible for NRPS to recognize more than one amino acid as its substrate (Ackerley \& Lamont, 2003). If the specificity is high, consistencies between metabolites occurs and results in the formation of a chemical group based on partial amino acid sequences or shared non-proteinaceous amino acids. If the amino acid specificity of the enzyme is low, this generates a highly variable position within the metabolite and thus increases structural diversity.

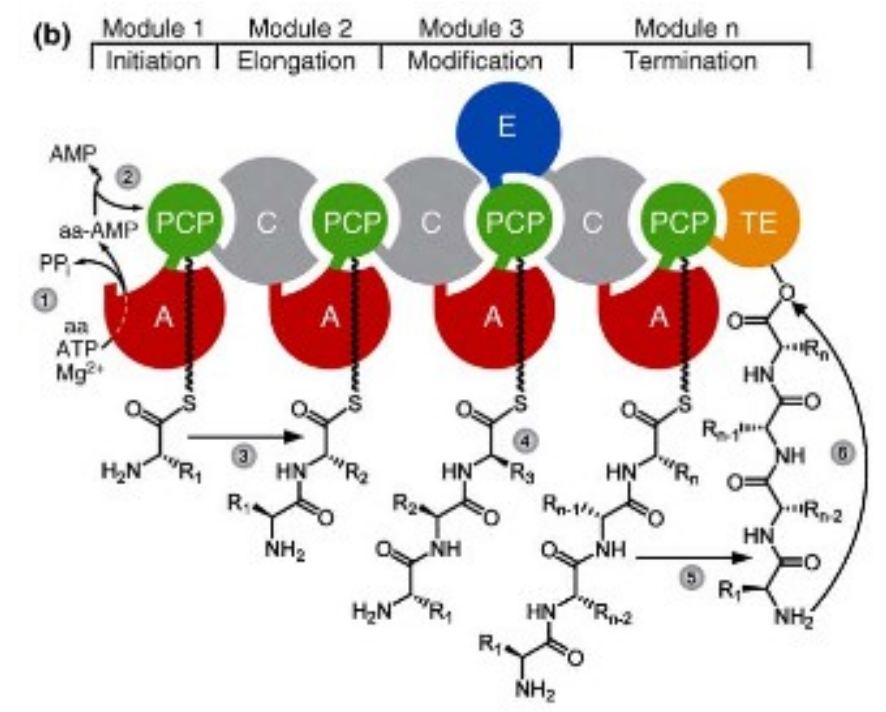

Figure 1.3: Simplified mechanism of non-ribosomal peptide (NRP) synthesis. (1) The amino acid is activated by the adenylation domain. (2) Transfer of the amino acid onto the PCP domain.

(3) Condensation of PCP-bound amino acids. (4) Possibility of amino acid modifications, for example by epimerization domains. (5) Transesterification of the peptide chain from the terminal PCP onto the TE domain. (6) TE domain catalyzed product release by either hydrolysis, 
aminolysis, or macrocyclization. The number of modification domains and modules is very variable (Strieker et al., 2010).

The majority of ribosomal peptides from RiPP pathways begin as precursor peptides with an Nterminal leader that guides biosynthetic enzymes responsible for post translational modifications to the core peptide which is eventually cleaved with a protease (Zhang, Chen, et al., 2018). Similar to proteins, these metabolites are synthesized ribosomally, passed along through various enzymatic domains of the synthase system matching an amino acid to a three-letter codon. As these metabolites have a ribosomal origin, their synthesis and structure may be mapped by analyzing the genome of the cyanobacteria, specifically the genes encoding the A domain enzyme. Structural diversity of RiPP synthesized cyanopeptides arises through post-translational modifications catalyzed by methyl-transferases, halogenases, and dehydrogenases.

\section{$\underline{1.3 \text { Cyanotoxins }}$}

Of the many bloom-forming genera, not all populations pose a health risk via the production of toxic metabolites. It is suggested that $25-75 \%$ of surface blooms are toxic (Zurawell et al., 2005). Toxic cyanobacteria are defined by the induction of harmful effects on vertebrates and humans and include species within the genera Microcystis (studied here), Dolichospermum (formerly Anabaena), Lyngbya, Cylindrospermopsis, Aphanizomenon, and Planktothrix. These genera produce mixtures of cyanotoxins that have neurotoxic, cytotoxic, and/or hepatotoxic effects (Pick, 2016; Zurawell et al., 2005). Toxic species of cyanobacteria produce complex mixtures of cyanotoxins in a strain-specific manner which results in vast structural diversity among cyanobacterial natural products (A. L. Demain \& Fang, 2000). Chemotaxonomy is the subclassification of strains within the same genus based on their chemical profiles. Understanding 
the metabolites that are generated by strains within the same genus can help determine genetic similarities and evolutionary divergence for classification.

\subsubsection{Neurotoxic cyanotoxins}

The major cyanotoxins with neurotoxic activity are alkaloid metabolites including anatoxins, also known as the Very Fast Death Factor because of the rapid physiological response, and the toxins associated with paralytic shellfish poisoning (PSP), saxitoxins (Figure 1.3, Yadav et al., 2011). Saxitoxins (STXs) are alkaloid natural products produced by species within the genera Aphanizomenon, Dolichospermum, Lyngbya, and Cylindrospermopsis but primarily by dinoflagellates in the genus Alexandrium (Testai et al., 2016). These tricyclic alkaloids may be sulfated or non-sulfated and possess a charged guanidine functionality resulting in potent neurotoxicity through binding to voltage-gated sodium ion channels in nerve cells. The resulting biological response causes numbness, paralysis, and death in mammals through respiratory arrest (Yadav et al., 2011). Saxitoxins have been shown to bioaccumulate throughout the marine food web, particularly shellfish, starting by consumption of algal cells by zooplankton and moving into higher organisms. Toxicity analysis of saxitoxins reveals an $\mathrm{LD}_{50}$ with intraperitoneal injection in mice of $10 \mu \mathrm{g} \mathrm{kg}^{-1} \mathrm{BW}$ and environmental concentrations reaching $193 \mu \mathrm{g} \mathrm{L}^{-1}$ in Washington State with some species reaching a toxin quota per cell of 0.03-1.30 pg cell-1 (Testai et al., 2016).

Anatoxins (ATXs) are produced by both benthic and brackish cyanobacteria species within the following genera Anabaena, Oscillatoria, Aphanizomenon, Cylindrospermum, and Phormidium (Figure 1.3, (Testai et al., 2016)). ATXs are bicyclic amine alkaloids with acute neurotoxicity through depolarization of neuromuscular blocking agents and provoking fatal asphyxia (Méjean et al., 2014). Prolonged exposure to the toxin results in muscle paralysis with the minimum lethal dose of anatoxin-a residing at $0.25 \mathrm{mg} \mathrm{kg}^{-1} \mathrm{BW}$ with intraperitoneal injection in mice. Mortality 
events attracted attention when in 2018 several dogs died in New Brunswick, Canada after ingesting large quantities of the toxin near the Saint John River. Depending on the species, as well as the strain of cyanobacteria, ATX is typically found in larger proportions intracellularly but has been detected within the environment extracellularly in concentration up to $1170 \mu \mathrm{g} \mathrm{L}^{-1}$ in Washington State (Testai et al., 2016).

$\beta$-N-methylamino-L-alanine (BMAA) is a modified non-proteinaceous amino acid produced by many cyanobacteria genera (Figure 1.3). This molecule was first detected in 1967 in the seed of a cycad tree and it has then been theorized that biomagnification of BMAA through the different trophic levels began with a cyanobacterial endosymbiont residing within the roots of the Cycas sp. (Botana et al., 2015). Since the initial discovery, the toxin has been reported to be produced by both symbionts and free-living species in all types of habitats, including marine, brackish, and freshwater. Like saxitoxins, this molecule bioaccumulates in fish and shellfish resulting in human exposure through consumption of contaminated seafood. The neurotoxicity of this molecule is complicated with various mechanisms within the cell such as, glutamate excitotoxicity, endoplasmic reticulum (ER) stress, mitochondrial dysfunction, oxidative damage, and toxic protein aggregation leading to motor deficits, neuronal degradation and somatic death (Tan et al., 2018). Long term with low dose exposures of this toxin has been correlated with neurodegenerative diseases such as Parkinson's and Alzheimer's disease with the median $\mathrm{LD}_{50}$ with intraperitoneal injection in mice being $3 \mu \mathrm{g} \mathrm{kg}^{-1} \mathrm{BW}$ (Al-Sammak et al., 2015). 

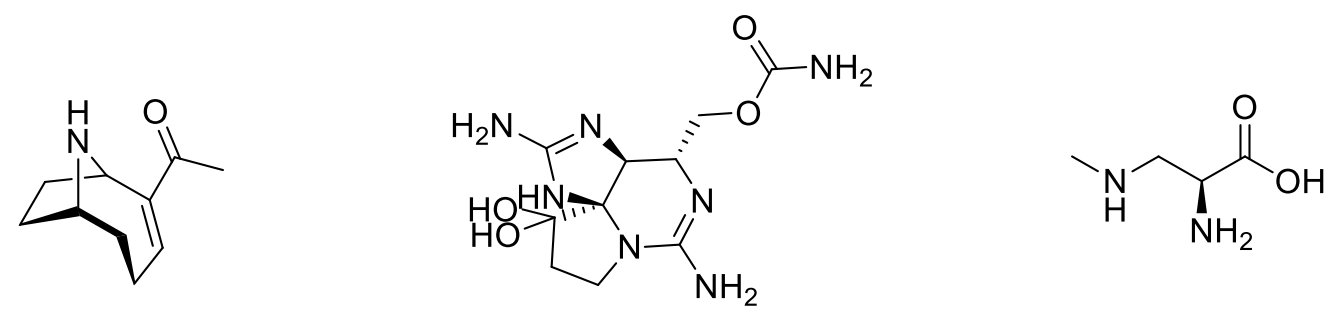

Figure 1.3: Chemical structures of neurotoxin cyanotoxins, anatoxin-a (left), saxitoxin (centre), and BMAA (right).

\subsubsection{Hepatotoxic cyanotoxins}

The alkaloid hepatotoxin cylindrospermopsin (CYL) is produced by several cyanobacteria genera including Anabaena, Aphanizomenon, Raphidiopsis, Lyngbya, and Cylindrospermopsis (Botana et al., 2015). CYL contains a tricyclic guanidine group with a sulfate group at one end and an uracil at the other end (Figure 1.4). CYL and isomers of this metabolite are biosynthesized through the PKS and NRPS pathways and organisms with these biosynthetic gene clusters are more commonly found in temperate climates (Botana et al., 2015). Bioaccumulation of this toxin occurs in many vertebrates and invertebrates within muscle and hepatopancreatic tissues. Humans are exposed to CYL via consumption of contaminated drinking water or fish. This results in hepatoenteritis as the toxin is distributed to the liver rapidly. In vitro studies with intraperitoneal injection of CYL to mice resulted in necrosis or apoptosis depending on the dose and the exposure time, with an $\mathrm{LD}_{50}$ of $2 \mathrm{mg} \mathrm{kg}^{-1} \mathrm{BW}$ after 24 hours and $0.2 \mathrm{mg} \mathrm{kg}^{-1} \mathrm{BW}$ if administered for 4-6 days (Botana et al., 2015). This data suggests that chronic low dose exposure is more toxic than administration of a larger single dose of CYL. 


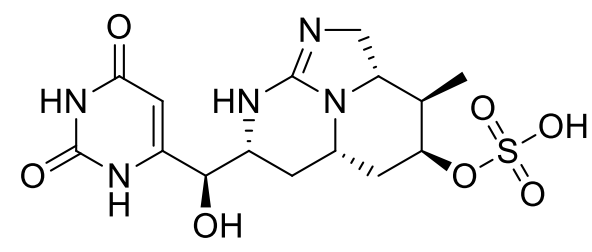

Figure 1.4: Chemical structure of cylindrospermopsin.

Cyanotoxins synthesized from NRPS synthesis pathways have the highest reports of hepatotoxicity for all previously reported cyanotoxins (Zurawell et al., 2005). The main two groups of cyanobacteria hepatotoxins are microcystins (MCs) and nodularins (NODs) which damage hepatocytes of the liver with death occurring in extreme cases due to intrahepatic hemorrhaging (Yadav et al., 2011). NODs are cyclic pentapeptides produced by species from the genus Nodularia and contain the diagnostic non-proteinaceous amino acid moieties 3-amino-9-methoxy-10-phenyl2,6,8-trimethyldeca-4,6-dienoic acid (Adda, red) and 2-(methylamino)-2-dihydrobutyric acid (Mdhb, blue) (Figure 1.5). The structural similarities between MCs and NODs include the Adda moiety, an NMe-Asp, and D-Glu. The primary difference between NODs and MCs is that are pentapeptides and MCs are hexapeptides. Another major difference is that NODs incorporate an Mdhb moiety and MCs incorporate a methyl-dihydroalanine (Mdha, green, Figure 1.5). The Adda moiety present in both these peptide variants results in inhibition of eukaryotic protein phosphatase 1 (PP1) and 2A (PP2A). Although, the substitution of Mdhb to Mdha in MCs results in an increased toxicity as the double bond within Mdha acts as a nucleophile and covalently bonds within the active sites of PPs (Zong et al., 2017). Microcystin congeners and toxicity will be discussed in greater detail in section 1.4.1. 


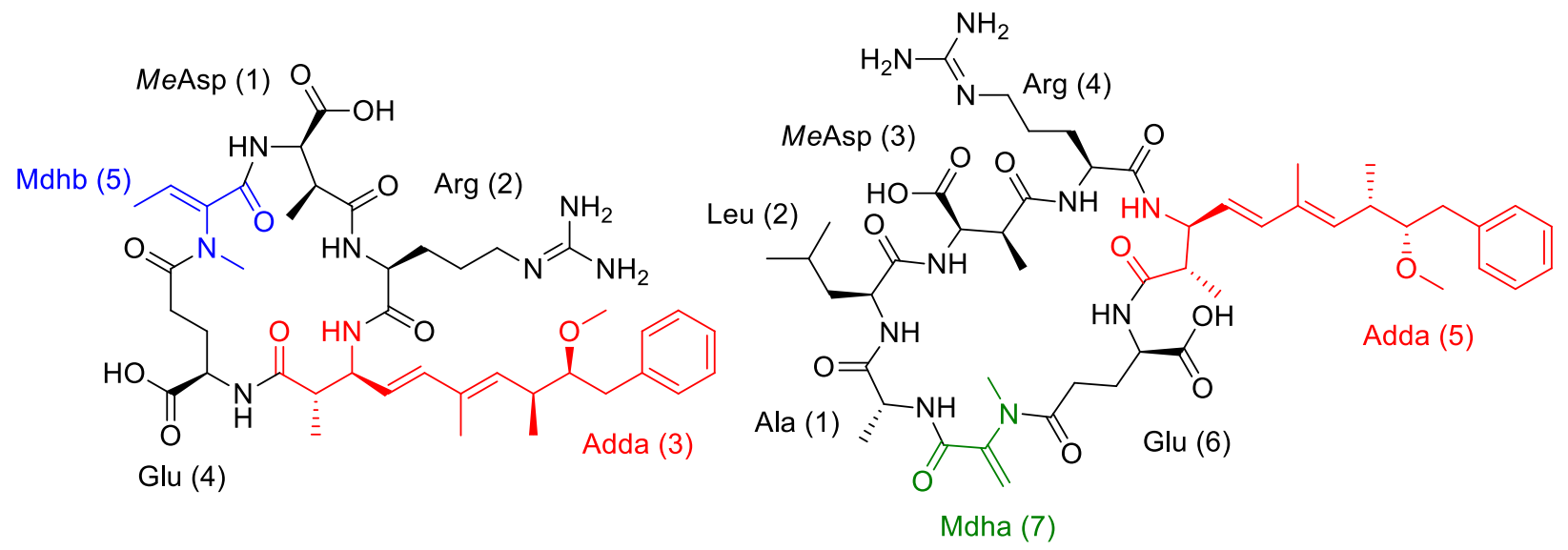

Figure 1.5: Chemical structure of hepatotoxins, nodularin-R (left) and microcystin-LR (right).

\section{$\underline{1.4 \text { Cyanopeptides from Microcystis species }}$}

Here metabolites biosynthesized by Microcystis sp. with NRPS or combined PKS-NRPS pathways are described. Most cyanopeptides are cyclic or linear non-ribosomal peptides that possess unique non-proteogenic amino acids and are grouped based on common structural features (Welker et al., 2006). Variable NRPS A domain sequences and tailoring enzymes are observed in studied gene clusters, even within strains of the same species suggesting unique metabolomic profiles for cyanobacteria strains. The variation in biosynthetic machinery also provides increased structural diversity in cyanopeptides and the ecotoxicology and toxicokinetics of the vast majority of cyanopeptides are not completely understood.

\subsubsection{Microcystins}

MCs are a group of more than 300 potent hepatotoxins and possible human carcinogens (IARC Group 2B, (Harke et al., 2016)). MCs are the most studied cyanopeptide group due to their potent biological activities and are known to be produced by several cyanobacterial genera such as Microcystis, Planktothrix, and Dolichospermum (Chernoff et al., 2020). MCs are cyclic 
heptapeptides that inhibit eukaryotic protein phosphatases 1 and 2A (PP1 and PP2A, De Figueiredo et al., 2004). The structural features that contribute to MC mode of action are the two non-protein amino acids that characterize this chemical class, Adda (3-amino-9-methoxy-10phenyl-2,6,8-trimethyldeca-4,6-dienoic acid, red) and Mdha (methyl-dehydroalanine, green, Figure 1.6). The long hydrophobic Adda moiety aids in reversible interactions that move the molecule into the pocket of the PP active site while the Mdha moiety interacts irreversibly within the active site by covalently bonding to a nucleophilic site within the pocket (Zong et al., 2017). Both these interactions result in inhibition of the catalytic activity of the phosphatases. Within hepatic cells, this results in hyperphosphorylation of key signal transduction proteins promoting oxidative damage, which can have several deleterious effects on the cell and organ such as cell structure disruption, apoptosis, liver necrosis, and intrahepatic hemorrhage (Zong et al., 2017).

There are two main variable positions within MC congeners, residues 2 and 4 which contribute to the naming convention for the over 300 reported congeners. MC-LR is the most studied congener within this class and has a Leu in position 2 and an Arg in position 4 (Figure 1.6). Environmental concentrations of total MCs can reach $150 \mu \mathrm{g} \mathrm{L}^{-1}$ and are not always the most dominant cyanopeptide group present (Janssen, 2019). MC-LR is the only MC congener that has a provisional guideline set by the World Health Organization (WHO) at $1.0 \mu \mathrm{g} \mathrm{L}{ }^{-1}$ in drinking water and a recreational water guideline of $20 \mu \mathrm{g} \mathrm{L}^{-1}$ or 100,000 cells $\mathrm{mL}^{-1}$ set by the Government of Canada. The regulation of only MC-LR poses a problem as MCs are typically produced as mixtures of different MC variants, with some cyanobacteria species producing exclusively other MC congeners. Thus, the total MC levels within drinking and recreational waters may be greatly underestimated. 


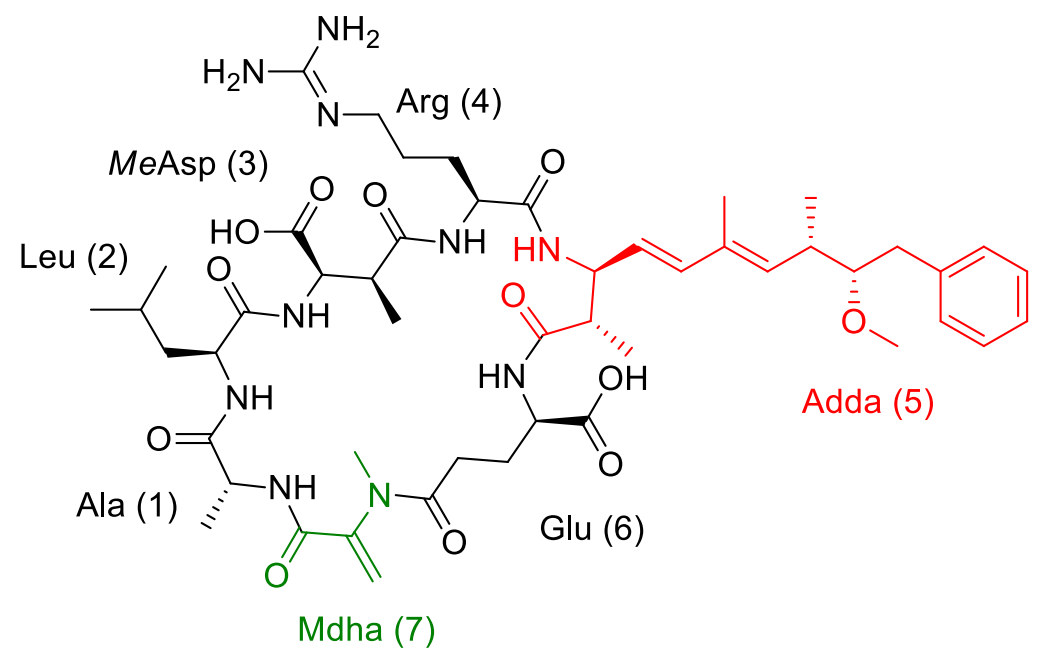

Figure 1.6: Chemical structure of the microcystin congener MC-LR.

There are few investigations of the toxicokinetics of MC congeners, with the main experimental focus on the major microcystins such as MC-LR. This congener is highly hydrophilic due to the arginine within position 4. Lipophilic congeners with hydrophobic residues within position 4 such as MC-LW and MC-LA, have significantly different toxicokinetics, such as uptake, tissue distribution, and excretion processes (Díez-Quijada et al., 2019). Absorption of MCs is achieved with organic anion transporting polypeptides (OATPs) and distribution to various organs is done rapidly within the blood stream. It has been shown that the higher lipophilicity of the MC congener results in a greater inhibition of PP2A than hydrophilic congeners due to a more efficient transport of the cyanopeptide into the cell (Feurstein et al., 2010). The hydrophobic congeners have also been shown to interact with bilayer membranes suggesting a secondary route into the cell. Experimental models have been investigated to assess the toxicity changes when substitution occurs in position 2 and 4. It was found in mice that the lethal dose was the same for MC-LR and MC-LA with a larger concentration required to achieve a lethal dose for MC-LY and MC-RR (Díez-Quijada et al., 2019). Alternatively, in the protozoan Tetrahymena pyriformis there were greater impacts on growth rate when treated with MC-LF and MC-LW in comparison to MC-LY 
and MC-LR. This suggests that the experimental model also has a large impact on the toxicity of each MC variant.

\subsubsection{Anabaenopeptins}

Anabaenopeptins are a class of hexapeptides that share a ureido linkage for cyclization with a Lys residue. The remaining amino acids are highly variable and result in vast structural diversity of the congeners within this chemical class (>100 reported, (Spoof et al., 2016)). These metabolites are synthesized via the NRPS pathway and were first isolated from Dolichospermum (Janssen, 2019). There are many subclasses of anabaenopeptins named according to the different genera of cyanobacteria which produce them such as oscillamides isolated from Planktothrix (Oscillatoria), ferintoic acids from Microcystis (Figure 1.7), nodulapeptins from Nodularia, and lyngbyaureidamides isolated from Lyngbya sp. (Spoof et al., 2016). It should be noted that these metabolites are produced by both marine as well as fresh-water cyanobacteria, further complicating the vast chemical diversity of this class of metabolites.

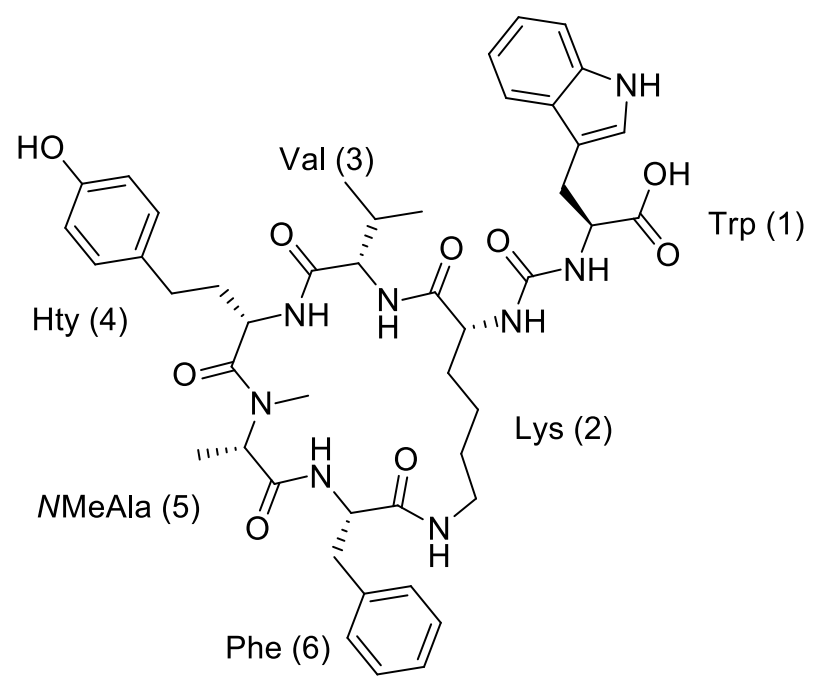

Figure 1.7: Structure of the anabaenopeptin congener produced by Microcystis sp., Ferintoic acid A. 
Anabaenopeptin congeners have shown enzyme inhibition against zinc-containing metalloexopeptidases, serine endopeptidases, as well as serine and threonine protein phosphatases (Spoof et al., 2016). The strength and specificity of the enzyme inhibition varies based on classification and amino acid sequence of the cyclic peptide. The concentration of some anabaenopeptins can reach concentrations similar to microcystins in the environment (Janssen, 2019).

\subsubsection{Cyanopeptolins}

Cyanopeptolins are cyclic depsipeptides containing a 3-amino-6-hydroxy-2-piperidone (Ahp, red, Figure 1.8) moiety at position 4. Position 2 is filled with an $\mathrm{NMe}$-aromatic amino acid such as Phe and Tyr, with possible halogenation of the Tyr (blue). The remainder of the molecule is highly variable, with an exo position containing two or more substituents typically filled with two amino acids or a fatty acid, such as hexanoic acid. Collectively, cyanopeptolins are potent serine protease inhibitors including trypsin, chymotrypsin, plasmin, thrombin, and elastase (Huang \& Zimba, 2019; Janssen, 2019). Structure-activity relationship revealed that the Ahp and residue 5 are important for protease inhibition specificity and potency (Linington et al., 2008). The cyanopeptolin class can be further broken down into subclasses based on the amino acids in position 2 and 3, beside the shared Ahp moiety. 

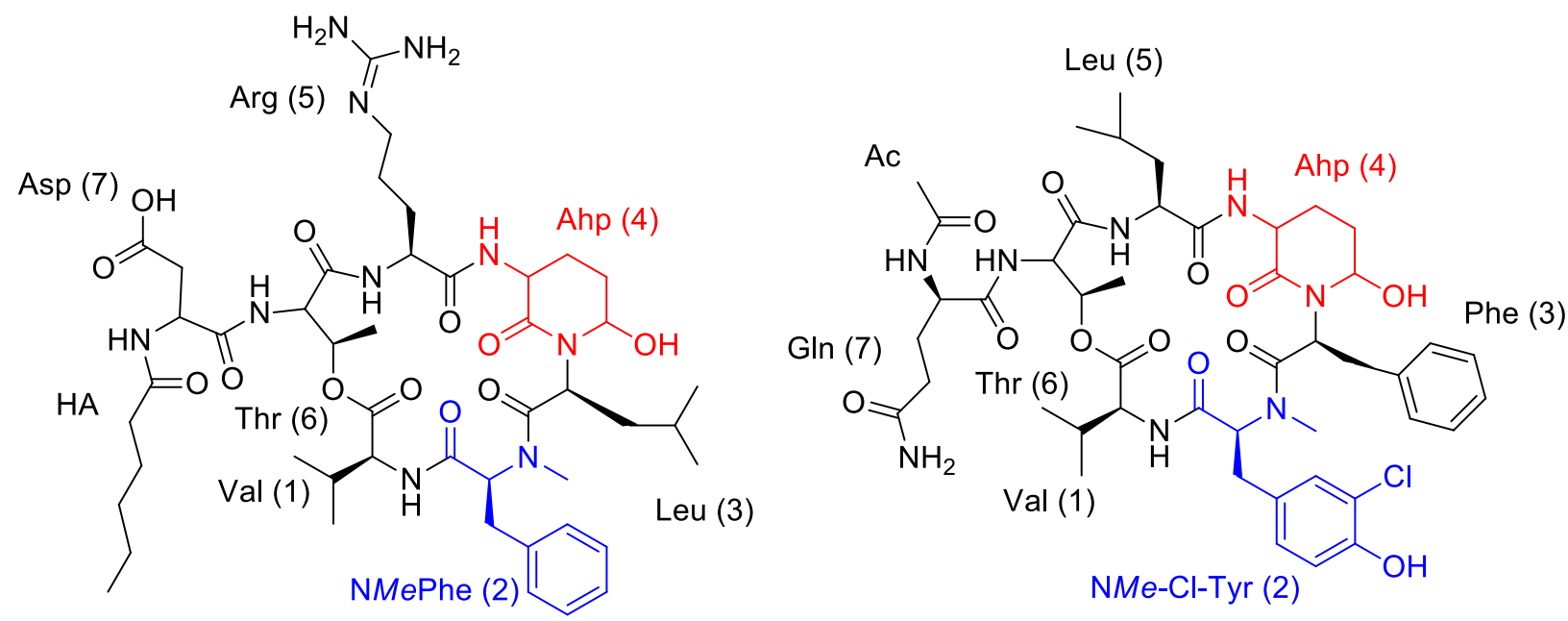

Figure 1.8: Chemical structure of cyanopeptolin A (left) and cyanopeptolin 954 (right). HxA; hexanoic acid, Ac; acetyl.

Fifty-one $\%$ of reported cyanopeptolin congeners have the sequence Lxx-Ahp, 32\% have the sequence Phe-Ahp, and 17\% are substituted with other proteinaceous amino acids, generated the three subclasses of cyanopeptolins (McDonald et al., 2020). Cyanopeptolin A is an example a LxxAhp cyanopeptolin and cyanopeptolin 954 is an example of a Phe-Ahp cyanopeptolin (Figure 1.8). Position 2 is filled with $\mathrm{NMePhe}$ in $31 \%$ of reported congeners and an NMeTyr in $36 \%$. The remainder of reported congeners have NMeTrp or methylated and/or halogenated NMeTyr. Reported environmental concentrations of these metabolites are similar to anabaenopeptins and microcystins, with production closely related to cell abundance (Janssen, 2019).

\subsubsection{Microginins}

Microginins are a class of linear N-acyl lipopeptides with four to six amino acids with a 3-amino2-hydroxy decanoic acid (Ahda, red, Figure 1.9) in the N-terminal position from a PKS pathway. Structural variants arise by modification or substitution of the N-terminal moiety to an octanoic acid derivative (3-amino-2-hydroxy octanoic acid, Ahoa, blue), N-methylation of the amino, halogenation of the acyl chain terminus, and the identity and number of other proteogenic amino 
acid building blocks. Other variants within this group have been named oscillaginins and nostoginins, with the naming convention derived by the genus that produces it, Oscillatoria and Nostoc, respectively (Strangman \& Wright, 2016).
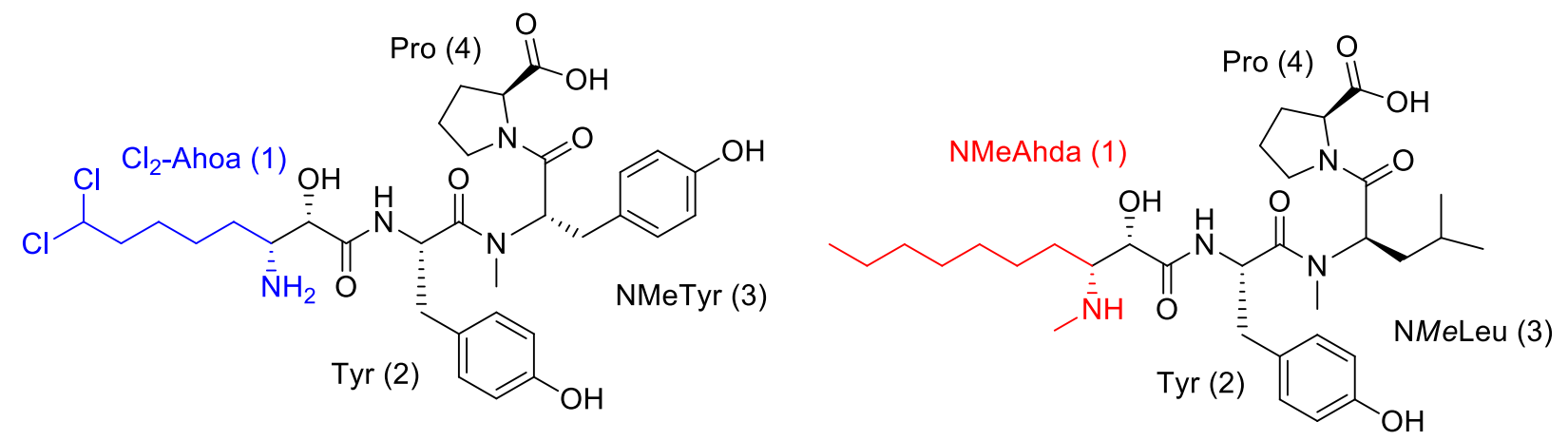

Figure 1.9: Chemical structure of microginin 680 (left) and microginin KR 604 (right).

The Ahda moiety is more prevalent in the $>90$ reported microginin congeners, making up $69 \%$ with the Ahoa moiety only $6 \%$. Chlorination of the terminal carbon occurs in the reported congener Microginin 680 and methylation of the amino functionality occurs in the reported congener Microginin KR604 (Figure 1.9). Position 3 contains an NMe-amino acid, with no specificity towards the chemistry of the substituted amino acid seen with the substitution from NMeTyr to NMeLeu (Figure 1.9). This chemical class has shown protease inhibition specifically against ACE and aminopeptidase at low $\mu \mathrm{mol} \mathrm{L} \mathrm{L}^{-1}$ concentrations (Keishi Ishida et al., 2000).

\subsubsection{Cyanobactins}

Cyanobactins are a large family of ribosomally synthesized cyanopeptides that undergo a range of post-translational modifications described below (Leikoski et al., 2013). Cyanobactin gene clusters are commonly found within the following cyanobacteria genera: Oscillatoria, Arthrospira, and Microcystis. The term cyanobactin encapsulates cyclic peptides within heterocyclized amino acids and further grouped into tenuecyclamides, trichamides, lyngbyactins, microcyclamides, 
aerucyclamide, aeruginosamides, and anacyclamides. Many cyanobacteria genera produce these complex toxins with over a hundred variants identified (Portmann, Blom, Kaiser, et al., 2008). The heterocycles and the quantity within the molecule dictate which subclass it falls within as well as the number and sequence of proteogenic amino acids. Cyanobactins often show cytotoxicity against different cancer cell lines but have also shown antiviral, antimalarial, and allelopathic activities (Leikoski et al., 2013). The five-membered heterocycles are formed from heterocyclization of Thr, Ser, and Cys into oxazoline and thiazolines. These residues can be further oxidized to oxazoles and thiazoles which occur in majority of the reported cyanbactins, increasing structural diversity of this chemical group (Ge et al., 2019).

Cyclic cyanobactins typically possess six residues, three proteinaceous amino acids and three heterocycles in alternating order. Variation arises with substitution of the proteinaceous amino acids as well as the heteroatoms within the heterocycle and the oxidation state of the heterocycle. For example, Meoxazolines are seen within aerucyclamides whereas the oxidized Meoxazoles are seen within microcyclamides (Figure 1.10). There are many enzymes attributed to the synthesis and post-translational modification to generate these molecules and results in large cyanobactin diversity.
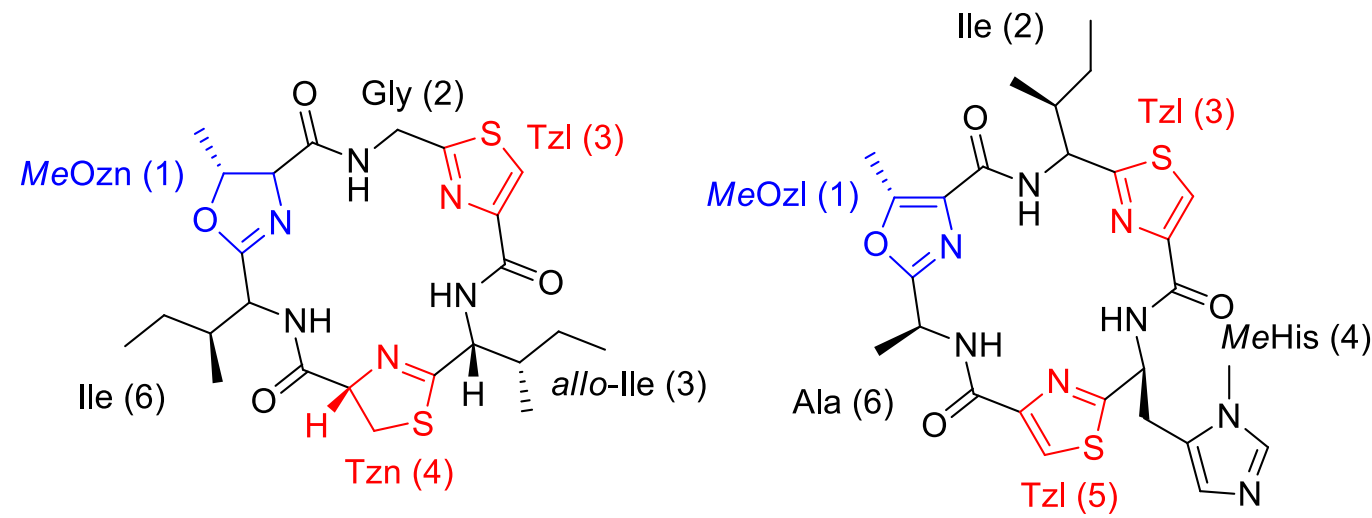

Figure 1.10: Chemical structure of aerucyclamide A (left) and microcyclamide A (right). 
Linear cyanobactins have similar heterocycles as cyclic cyanobactins although post-translational modifications make cyclization impossible (Leikoski et al., 2013). In the case of aeruginosamides B and C, the C-terminal thiazole (Thz) is capped with a methyl ester (thiazole carboxylate, Tzc, blue) preventing $\mathrm{C}$ - to $\mathrm{N}$ - cyclization (Figure 1.11). These congeners typically have a 4 to 5 amino acid sequence with the unique 1,1-dimethylallyl moiety (DmA, red) at the N-terminus. This particular variety of cyanobactins has prenylated $\mathrm{N}$-terminus and O-methylated C-terminus.
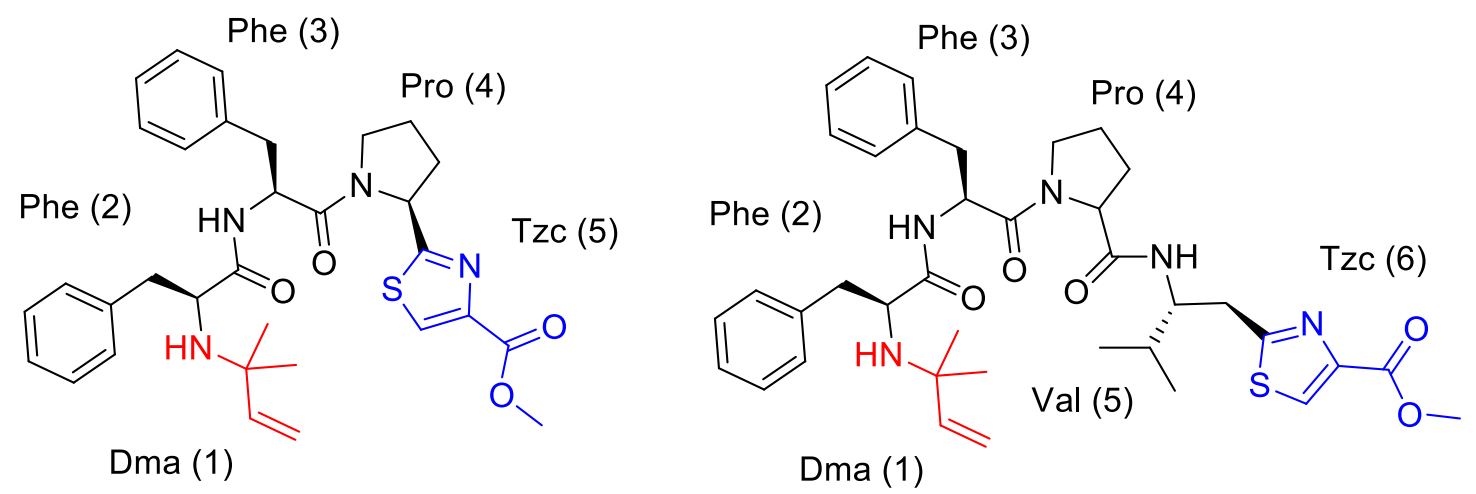

Figure 1.11: Chemical structure of aeruginosamide B (left) and C (right).

\subsubsection{Aeruginosins}

Aeruginosins are small linear tetrapeptides that contain the unique non-proteinaceous amino acid 2-carboxy-6-hydroxyoctahydroindole (Choi, red, Figure 1.12) and a C-terminal Arg or cyclized Arg, namely Argal. The hydroxyl of the Choi functionality can be functionalized with sulfates, phosphates, and other moieties. Another characteristic features of aeruginosins is an $\mathrm{N}$-terminal hydroxy-phenyllactic acid (Hpla), which may undergo halogenation such as bromination and chlorination (Welker et al., 2006). 


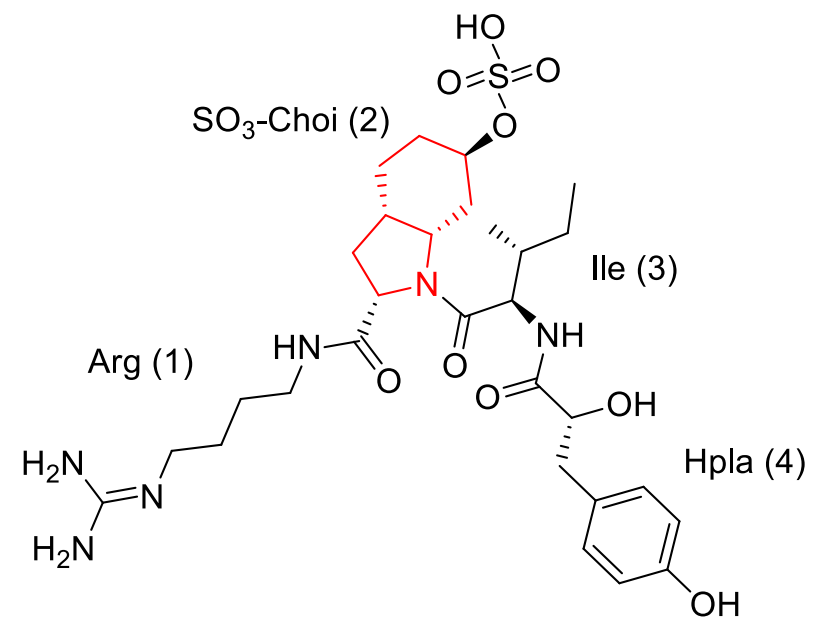

Figure 1.12: Chemical structure of aeruginosin 98A.

Aeruginosins are known protease inhibitors like variants of the cyanopeptolin group (Elkobi-Peer \& Carmeli, 2015). These metabolites typically appear in structurally related mixtures of compounds with substitutions of the proteinaceous amino acids in position 3 (Figure 1.12). Several cyanobacteria genera produce aeruginosin like compounds such as Microcystis, Oscillatoria, Nodularia, and Planktothrix (Yadav et al., 2011).

\subsubsection{Microviridins}

Microviridins are a complex cyanopeptide group with three peptide chains linked together by $\omega$ ester and $\omega$-amide linkages (Ziemert et al., 2010). These peptides are typically between 1400-2000 Da, with 14 amino acids, and are synthesized through the RiPP synthesis pathway implying that the amino acid building blocks are proteinaceous (Figure 1.13). Without characteristic nonproteinaceous amino acids, classifying this chemical class is solely based on the presence of uncommon proteogenic amino acids such as Trp as well as with a retention time range and identification of doubly and triply charged precursor ions. 


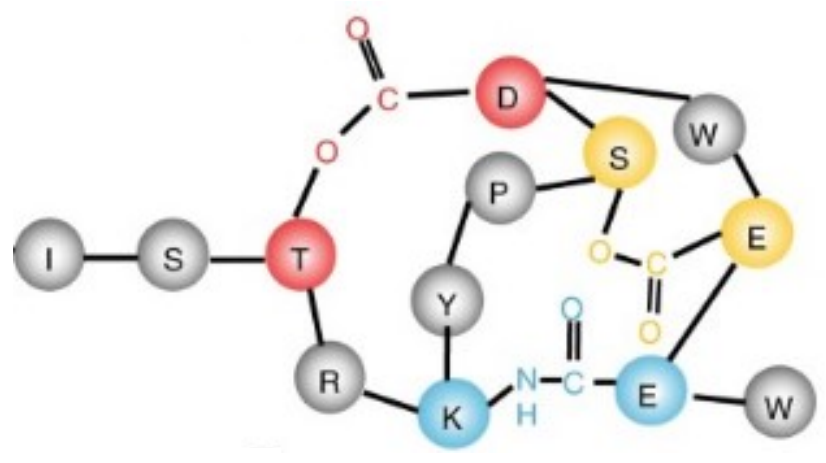

Figure 1.13: Amino acid depiction for the chemical structure of microviridin N.

Most microviridin variants show enzyme inhibition activity against serine-type proteases (Ziemert et al., 2010). One variant, microviridin $\mathrm{J}$, is unique due to the substitution of Arg between the Thr and Lys residues which has been suggested to be the key binding site for toxicity as this congener has potent trypsin inhibition (Rohrlack et al., 2003). Other microviridin congeners, such as microviridins B, C, G, H, I have a Leu in the position between the Thr and Lys and show very potent elastase inhibition whereas those with Met, Tyr, or Phe (microviridin D, E, F) in between the residues Thr and Lys show little to no elastase inhibition activity (Rohrlack et al., 2003).

The genetic machinery required for synthesis of these large cage-like proteins include many enzymes such as ligases, acetyl-transferases, and those for lactonization and cyclization (Ziemert et al., 2010). With a large number of amino acids and enzyme complexes involved in microviridin synthesis, there are many opportunities for modification and amino acid substitutions leading to high chemical variation within this class.

\subsection{Metabolomics}

Metabolomics is the comprehensive study of all metabolites produced by an organism, known as the metabolome (Tiam et al., 2019). Metabolomics employs complex analytical instrumentation 
in conjunction with post-data acquisition techniques to monitor and discover metabolic changes in biological systems (Beger, 2013). It has become an important method in recent years to decipher the metabolites that unite and differentiate products of both primary and secondary metabolic pathways based on experimental treatments, cell types, and strains. There are targeted metabolomic methods that require standards for quantification and a predetermined list of known metabolites that are utilized to assess shifts in well-defined metabolic pathways and biological systems. Conversely, there are untargeted metabolomic methods which visualize and interpret an entire chemical profile by gathering information on all metabolites that meet pre-defined intensity parameters (Beger, 2013). With a large untargeted dataset, several metabolite profiling platforms can be used for the rapid identification of previously reported metabolites and potential new metabolites. Untargeted metabolomics is becoming a more common strategy in secondary metabolite investigations and is key for the identification of novel metabolites as potential drug candidates (Tiam et al., 2019). Expansion of chemical libraries with experimental chromatographic and spectral data will aid in rapid dereplication of metabolites within a complex sample and highlight potential new compounds.

\subsubsection{High-resolution LC-MS data collection}

The analysis of complex samples that contain hundreds of chemicals is possible by combining several chemistry techniques. With advances in chromatography and mass spectrometry (MS) instrumentation, comprehension of complex biological extracts can be achieved. Through the use of sophisticated mass analyzers such as orbitraps or quadrupole time-of-flight (QToF), untargeted data collection provides high-resolution mass data for all ionizable chemicals within a sample (Nagana Gowda \& Djukovic, 2014). This first requires chromatographic separation of the sample, gas or liquid, followed by high-resolution mass spectrometry (HR-MS) and tandem mass 
spectrometry (MS/MS). This provides chemical information for each ionizable metabolite such as a retention time (relates to metabolite polarity), precursor ion $\mathrm{m} / \mathrm{z}$, as well as a number of product ions generated by colliding the molecule with inert gas and detecting the fragments, which are direct clues to the structure of the metabolite. The method used in this investigation is liquid chromatography high-resolution mass spectrometry (LC-HRMS).

Ionization of the metabolites within a sample is one of the most important steps of MS measurements. The most often used methods are electrospray ionization (ESI) and electron impact (EI) ionization (Nagana Gowda \& Djukovic, 2014). In LC-HRMS, ESI is the optimal ionization method and used here for multiple reasons. ESI is a soft ionization method and does not readily induce in-source fragments resulting in dominant molecular ions as well as it adequately ionizes both large and small biomolecules within the liquid phase. A drawback of ESI is the generation of salt adducts such as sodiated and ammoniated (Table 2.3) ions with the use of buffered solvent systems. However, in combination with other observed ions they can be confirmatory together.

The mass analyzer is capable of manipulating the ionized compounds and assigning mass to charge $(\mathrm{m} / \mathrm{z})$ ratios. Here, an Orbitrap single-configuration mass analyzer is used which provides highresolution mass spectral (HR-MS) data which is ideal for global profiling and metabolite identification. A data-dependent acquisition (DDA) experiment collects both HRMS and MS/MS spectra for ionized compounds that meet pre-defined criteria. The collection of HRMS/MS data is vital in the dereplication of previously identified compounds and classification of structurally related compounds as compounds with shared structural features will generate common product ions upon fragmentation.

HR-MS and HRMS/MS data provides isotopic analysis and a molecular formula based on the mass defect principle. Every atom has a unique mass defect, meaning their mass is slightly lower than 
the sum of the mass of their protons and neutrons. Thus a metabolite will have a characteristic mass defect corresponding to the number and type of atoms it contains (Dettmer et al., 2007). With the molecular formula for a chemical, identification by comparison to literature is simplified. However, isobaric compounds (i.e., compounds with the same molecular formula) cannot be differentiated. For example, the immonium ions generated for the amino acids leucine $(\mathrm{m} / z$ 86.0969) and isoleucine ( $m / z$ 86.0969), have the same $\mathrm{m} / \mathrm{z}$ and molecular formula and cannot be differentiated. Structural isomers and stereochemistry cannot be elucidated with HR-MS data. The challenge of discriminating compounds based on molecular formula alone is eased with comprehensive databases and libraries of previously reported common chemicals for rapid dereplication of natural products or establishing a metabolite as potentially novel.

\subsubsection{Untargeted mass-spectrometry based metabolomics}

The large HRMS and HRMS/MS dataset acquired as described in Section 1.5.1 must be interpreted with sophisticated post-data acquisition strategies to determine similarities and differences within chemical profiles. Multivariate analyses performed with R statistical software and the packages xcms, FactoMineR, and MetabolAnalyze allow for visualization and comprehension of the statistically significant features (compounds) that contribute to diversifying metabolomes and which are common to the investigated metabolomes. Within this investigation, multivariate statistical analyses was performed to assess Microcystis chemotypes for five strains, specifically principal component analysis (PCA), factor loading plot analysis, and Kruskal Wallis (KW) test for two or more groups of non-parametric data.

PCA is a means of describing the most pertinent variability within a multivariate dataset. PCA works to condense variation within a dataset into fewer dimensions and is thus desirable when working with massive multivariable datasets (Pearson, 1901). This statistical test is unsupervised 
and converts potentially correlated variables into sets of uncorrelated variables called principal components. Therefore, when samples are plotted closely along the principal components the variables that define them are more similar than samples plotted further away along the principal components. Further, to depict which variables distinguish and unite the samples, a factor loading plot may be generated. This type of plot depicts the contribution of each feature to the distribution of the samples within the PCA. Within LC-HRMS based metabolomics, these features are identified compounds detected by LC-HRMS. The KW test is performed on the features within the factor loading plot to provide statistical significance to the variables that define the distribution of the samples within the PCA. A Benjamini-Hochberg correction was utilized here to ensure false positives did not influence the analysis.

Metabolites with similar structural features such as amino acid sequences and/or nonproteinaceous amino acids will generate similar product ions when subjected to LC-HRMS/MS analysis. This peptidyl nature of cyanopeptides enables grouping of the secondary metabolites based on structural similarity and may be used for putative identification and characterization of new and known cyanopeptides. The related nature of these molecules based on their biosynthetic mechanism allows for several post-data acquisition untargeted metabolomic strategies to be applied to sort through the large data set and organize the chemicals that have been detected based on factors, such as structural similarity or which Microcystis strain produced them.

Another mode of processing the large untargeted LC-HRMS/MS data sets utilizes the recently developed global natural product social (GNPS) algorithm. This method compares all MS/MS spectra within the data file and generates a network with clusters of structurally related compounds that share product ion profiles (Tiam et al., 2019). Compounds that have similar structural features will generate common product ions within the HRMS/MS spectra and thus generate a cluster 
within the molecular network. The nature of the cyanopeptides studied here are an ideal set of compounds for this analysis as the building blocks that make up these metabolites are often shared. Also, as alluded to within previous sections, cyanopeptides are grouped that share unique structural features such as non-proteinaceous amino acids or partial amino acid sequences. With identification of known cyanopeptides within the molecular network, it is possible to identify related metabolites with minor structural modifications such as methylation, halogenation, and/or amino acid substitutions.

\subsubsection{Semi-targeted mass-spectrometry based metabolomics}

From the information gathered by searching LC-HRMS/MS raw data and the untargeted LCHRMS/MS data analysis, characteristic features for each chemical group such as retention time ranges, precursor ion $m / z$ ranges, and diagnostic product ions can be used to perform semi-targeted searches of LC-HRMS/MS datasets for any and all metabolites with a particular structural feature relating to a chemical group. A technique known as diagnostic fragmentation filtering (DFF) can be employed to take advantage of the structural similarity. Within the mass spectrometry data analysis software, mzMine, a feature known as diagnostic fragmentation filtering allows for the search of diagnostic product ion pairs or combinations which may be tailored to a specific chemical class (McMullin et al., 2019). The output contains all metabolites within the large data set that contain the specified product ions within their MS/MS.

Cyanobacteria produce diverse and complex mixtures of secondary metabolites that require complex analytical methods for detection and comprehension of the complete chemical profile. There is limited information on the toxicity and chemistry of the many cyanopeptide congeners. Small modifications such as methylation, halogenation, and single amino acid substitutions can have great impacts on the biological role of a molecule and fate within an organism and the 
environment. To gain understanding of the total chemical profile, all chemicals within a sample must be detected. Following this, implement a rapid method for the identification of new and dereplication of known chemicals.

\section{$\underline{1.6 \text { Project aim }}$}

Canada contains $20 \%$ of the global freshwater resources and in the last one hundred years, its freshwater ecosystems (lakes, rivers, and wetlands) have undergone widespread environmental change. The stress on ecosystems is due to of human population growth, pollution, increased temperatures and habitat degradation. A visible consequence of this environmental change is an increase in frequency and magnitude of cyanobacterial blooms. Positive correlations between toxic cyanobacteria and adverse health effects have been demonstrated in several countries including Canada. Toxic cyanobacteria populations produce diverse and strain-specific mixtures of cyanotoxins with potential hepatotoxic, cytotoxic, and neurotoxic effects. The biological factors that promote cyanobacteria growth and toxin production are broadly understood; however, the chemistry, biological activity, and environmental concentration for the majority of cyanopeptides have not been investigated. The objective of this study was to apply mass-spectrometry based metabolomics to decipher the strain-specific cyanopeptide profiles of five Microcystis strains. Multivariate analyses, including PCA and factor loading, are used to assess the metabolites that statistically contribute to Microcystis chemotype differences. Cyanopeptide structural diversity is investigated through GNPS molecular networking via groups of structurally related cyanopeptides that are co-produced by Microcystis strains. The information gathered regarding the different cyanopeptide groups is applied to environmental surface water and bloom samples collected in Ontario and Quebec for the description of cyanopeptide profiles found within these different ecosystems. Finally, the preliminary cyanopeptide identification will be used to investigate effects 
of media composition on Microcystis growth and metabolite production. This information will aid in the prioritization of target strains for large scale cultivation for the isolation and purification of novel cyanopeptides and common cyanopeptides contaminating the environment to undergo structural and toxicological characterizations.

\section{Materials and Methods}

\section{$\underline{2.1 \text { General experimental procedures. }}$}

Cyanobacteria cultures were grown under white light $\left(30 \mu \mathrm{M} \mathrm{m}^{-2} \mathrm{~s}^{-1}\right)$ in a Conviron E15 incubator with $40 \%$ humidity and a light/dark regime of $12 \mathrm{~h}$. Cyanobacterial growth was monitored via cell number (cells $\mathrm{mL}^{-1}$ ), counted with a Hausser Scientific Co. counting chamber (catalog \#3200) under a VistaVision Microscope. Optical density measurements were recorded with a Molecular Devices SpectraMax 340 PC 384 microplate reader. Cyanobacteria cells were harvested on Whatman glass microfiber filters (GF/A diameter $47 \mathrm{~mm}$, CAT No.1820-047) using a Millipore filtration apparatus. The extraction of intracellular cyanopeptides were performed in VWR disposable culture tubes $(16 \times 100 \mathrm{~mm})$. Vortex and sonication were conducted using a Fisher Scientific Vortex Mixer and Aquasonic VWR Scientific Products Model 50T. Resulting intracellular cyanopeptide extracts were filtered through PTFE syringe filters (Prefilter $30 \mathrm{~mm}$ $0.22 \mu \mathrm{m}$, model No. PTFEP030N022O). All solvents were purchased from VWR and chemicals for media were purchased from what was purchased from Eurofins Abraxis and performed according to the manufacturer's instructions. Optical density and absorbance for the PP2A inhibition assay were read with a Molecular Devices SpectraMax spectrophotometer in 96-well tissue culture microplates. Microcystin standards (MC-LR, [Asp³]-MC-LR, MC-RR, Nodularin) 
were received from the National Research Council in Nova Scotia, Canada and used for identification and quantification of microcystin congeners.

\section{$\underline{2.2 \text { Cyanobacteria strain cultivation. }}$}

Four Microcystis aeruginosa strains CPCC 299, CPCC 300, CPCC 632, CPCC 633 and one Microcystis flos-aquae strain CPCC 461 were obtained from the Canadian Physiological Culture Centre (CPCC) and selected for metabolomics as pre-screening revealed diverse chemical profiles. Collection data and specific growth rates in three defined cyanobacteria culture media are displayed in Table 2.1 for each studied strain. The three defined culture media (BG-11, MA, BBM) were selected because they are commonly used to grow Microcystis species. $25 \times$ stock solutions for these media were made according to their recipes (Table 2.2; Watanabe et al., 1996; Anderson 2005). Aliquots of the stock solution were diluted in $\mathrm{ddH}_{2} \mathrm{O}$ and autoclaved prior to inoculation with Microcystis cells.

Table 2.1: Cyanobacteria strain information from the Canadian Phycological Culture Collection.

\begin{tabular}{|c|c|c|}
\hline \multirow{2}{*}{ Strain } & \multicolumn{2}{|c|}{ Collection Data } \\
\cline { 2 - 3 } & Date & Location \\
\hline M. aeruginosa CPCC 299 & $08 / 90$ & Pretzlaff Pond AB, Canada \\
\hline M. aeruginosa CPCC 300 & $08 / 90$ & Pretzlaff Pond AB, Canada \\
\hline M. flos-aquae CPCC 461 & $08 / 75$ & Fox River WI, USA \\
\hline M. aeruginosa CPCC 632 & $10 / 48$ & Lake Mendota WI, USA \\
\hline M. aeruginosa CPCC 633 & $10 / 54$ & Little Rideau Lake, ON Canada \\
\hline
\end{tabular}

Each medium contains a different iron source but all have $\mathrm{NaNO}_{3}$ as the major nitrogen source. The differences between BBM and BG-11 media are mainly concentration based as majority of the chemicals used are the same with BG-11 containing double the concentration of $\mathrm{NaNO}_{3}(1.5 \mathrm{~g}$

$\left.\mathrm{L}^{-1}\right)$ compared to $\mathrm{BBM}\left(0.75 \mathrm{~g} \mathrm{~L}^{-1}\right)$. The main phosphorous source is $\mathrm{K}_{2} \mathrm{HPO}_{4}$ for $\mathrm{BBM}(0.175 \mathrm{~g}$ $\left.\mathrm{L}^{-1}\right)$ and BG-11 (0.03 $\left.\mathrm{g} \mathrm{L}^{-1}\right)$ and BBM has an additional phosphorous source, $\mathrm{KH}_{2} \mathrm{PO}_{4}\left(0.75 \mathrm{~g} \mathrm{~L}^{-1}\right)$. 
MA is much different than BBM and BG-11 with only a few overlapping chemicals, such as $\mathrm{NaNO}_{3}, \mathrm{Na}_{2} \mathrm{EDTA}$, and $\mathrm{H}_{3} \mathrm{BO}_{3}$, but has an additional nitrogen source being $\mathrm{KNO}_{3}$ and the major phosphorous source being $\beta-\mathrm{Na}_{2}$-glycerophosphate. These fluctuations in the vital nutrients for cyanobacterial growth are believed to impact the growth rate and subsequent toxin production.

All strains were inoculated into sterile media (Table 2.2) at high $10^{4}-$ low $10^{5}$ cells $\mathrm{mL}^{-1}$ and grown in $250 \mathrm{~mL}$ Erlenmeyer flasks (Pyrex) that contained $50 \mathrm{~mL}$ of defined culture media (Table 2.2). Cultures were grown in triplicate and maintained in a growth chamber (Conviron E15) under cool white fluorescent light $\left(30 \mu \mathrm{E} \mathrm{m} \mathrm{m}^{-2} \mathrm{~s}^{-1}\right)$ using a light/dark regime of $12 \mathrm{~h}$ (Figure 2.1). Cyanobacteria were grown at several different temperatures to assess optimal growth rates, however $25{ }^{\circ} \mathrm{C}$ was routinely used to grow cyanobacteria to study their cyanopeptide profiles. Flasks were swirled every two days and late-exponential cells were harvested after 15 days and extracted according to the procedure described in Section 2.4.

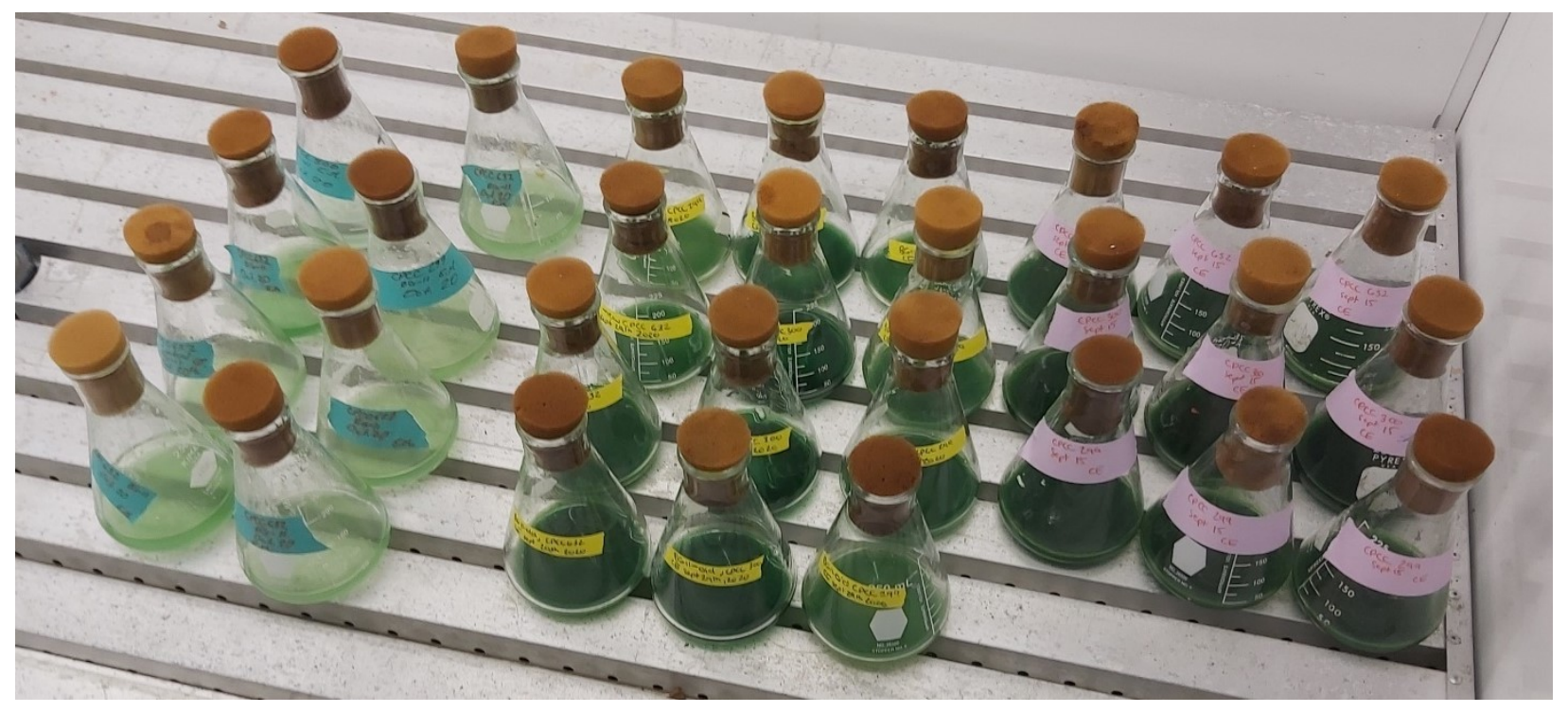

Figure 2.1: Microcystis strains growing in $250 \mathrm{~mL}$ Erlenmeyer flasks within the growth chamber. 
Each Microcystis strain studied was also grown in BG-11 medium supplemented with $\mathrm{Na}^{15} \mathrm{NO}_{3}$ (Sigma Aldridge) instead of $\mathrm{NaNO}_{3}$, which represents the major source of nitrogen in the medium (Andersen 2005). These cultures were grown for 20 days and subsequently transferred to fresh BG-11 media containing $\mathrm{Na}^{15} \mathrm{NO}_{3}$. The process was repeated four additional times and facilitated the biosynthesis of ${ }^{15} \mathrm{~N}$ labelled cyanopeptides by each strain. Strains were also grown as described above in BBM and MA media (Table 2.2), other common media used to Microcystis species (Anderson 2005).

Table 2.2: Media components for the defined culture media used to grow Microcystis species.

\begin{tabular}{|c|c|c|c|}
\hline \multirow[t]{2}{*}{ Component } & \multicolumn{3}{|c|}{ Final concentration $\left(\mathrm{g} \mathrm{L}^{-1}\right)$} \\
\hline & BBM & BG-11 & MA \\
\hline $\mathrm{MgSO}_{4} \bullet 7 \mathrm{H}_{2} \mathrm{O}$ & 0.075 & 0.075 & - \\
\hline $\mathrm{NaNO}_{3}$ & 0.75 & 1.5 & 0.05 \\
\hline $\mathrm{K}_{2} \mathrm{HPO}_{4}$ & 0.175 & 0.03 & - \\
\hline $\mathrm{Na}_{2}$ EDTA & 0.001 & 0.001 & 0.005 \\
\hline $\mathrm{CaCl} \bullet 2 \mathrm{H}_{2} \mathrm{O}$ & 0.025 & 0.036 & - \\
\hline $\mathrm{KH}_{2} \mathrm{PO}_{4}$ & 0.75 & - & - \\
\hline $\mathrm{NaCl}$ & 0.025 & - & - \\
\hline $\mathrm{FeSO}_{4} \bullet 7 \mathrm{H}_{2} \mathrm{O}$ & 0.00498 & - & - \\
\hline $\mathrm{H}_{3} \mathrm{BO}_{3}$ & 0.00011 & 0.00286 & 0.02 \\
\hline $\mathrm{MnCl}_{2} \bullet 4 \mathrm{H}_{2} \mathrm{O}$ & 0.00181 & 0.00181 & 0.005 \\
\hline $\mathrm{CuSO}_{4} \cdot 5 \mathrm{H}_{2} \mathrm{O}$ & 0.000079 & 0.000079 & - \\
\hline $\mathrm{ZnSO}_{4} \cdot 7 \mathrm{H}_{2} \mathrm{O}$ & 0.000222 & 0.000222 & - \\
\hline $\mathrm{Co}\left(\mathrm{NO}_{3}\right) 2 \bullet 6 \mathrm{H}_{2} \mathrm{O}$ & 0.0000494 & 0.0000494 & - \\
\hline $\mathrm{Na}_{2} \mathrm{MoO}_{4} \bullet 2 \mathrm{H}_{2} \mathrm{O}$ & 0.00039 & 0.00039 & 0.0008 \\
\hline Biotin & 0.000001 & 0.000001 & - \\
\hline Vitamin B12 & 0.000001 & 0.000001 & - \\
\hline Thiamine $\mathrm{HCl}$ & 0.0002 & 0.0002 & - \\
\hline Ferric ammonium citrate & - & 0.006 & - \\
\hline $\mathrm{MnSO}_{4} \cdot 7 \mathrm{H}_{2} \mathrm{O}$ & - & 0.075 & - \\
\hline $\mathrm{Na}_{2} \mathrm{CO}_{3}$ & - & 0.02 & - \\
\hline $\mathrm{FeCl}_{3} \bullet 6 \mathrm{H}_{2} \mathrm{O}$ & - & - & 0.0005 \\
\hline $\mathrm{Na}_{2} \mathrm{SO}_{3}$ & - & - & 0.04 \\
\hline $\mathrm{CoCl}_{2} \bullet 6 \mathrm{H}_{2} \mathrm{O}$ & - & - & 0.005 \\
\hline $\mathrm{KNO}_{3}$ & - & - & 0.1 \\
\hline$\beta-\mathrm{Na}_{2}$-glycerophosphate & - & - & 0.1 \\
\hline Bicine & - & - & 0.5 \\
\hline $\mathrm{ZnCl}_{2}$ & - & - & 0.0005 \\
\hline
\end{tabular}




\subsection{Monitoring the growth of Microcystis strains.}

To understand how the strain specific physiology and metabolism, as well as nutrient source and concentration impact Microcystis growth and toxin production, the strains were grown in the three defined culture media described in section 2.2. Biological growth indicators such as $\mathrm{pH}$, cell number, optical density, and dried biomass were recorded on day 5, 10, 15, 20, and 25 in triplicate. Toxin production was monitored with the same schedule via intracellular extraction and LCMS/MS analysis (Section 2.4 and 2.6). $\mathrm{pH}$ was read with a Fisher brand $\mathrm{pH}$ meter calibrated with $\mathrm{pH} 4,7$, and 10 buffer solutions. Cells $\mathrm{mL}^{-1}$ were counted using $10 \mu \mathrm{L}$ of culture on a counting chamber and visualized under a microscope. If the cultures were overgrown, the cells were diluted $10 \times$ with distilled deionized water in a glass vial. $150 \mu \mathrm{L}$ of undiluted culture was pipetted into a 96 well plate for an optical density reading at $750 \mathrm{~nm}$ for chlorophyll absorbance. Finally, $25 \mathrm{~mL}$ of culture was filtered onto dried and pre-weighed glass microfiber filter paper and left overnight at $40^{\circ} \mathrm{C}$. The dried filter paper was weighed the following day to obtain dried biomass. Specific growth rates $(\mu)$ were calculated using the cell $\mathrm{mL}^{-2}$ value at day $0\left(\mathrm{~N}_{0}\right)$ and the cell $\mathrm{mL}^{-1}$ value when the cells reached the lag phase. In all experiments, this was the 25 day reading $\left(\mathrm{N}_{25}\right)$. The following formula was used to provide a comparable specific growth rate with units, days ${ }^{-1}$ (Anderson, 2005).

$\mu=\ln \left(\mathrm{N}_{\mathrm{t}} / \mathrm{N}_{0}\right) / \Delta \mathrm{t}$

\subsection{Extraction of cyanopeptides from Microcystis cells.}

Strain specific differences and media composition effects on Microcystis toxin profiles were investigated by intracellular extraction of cyanopeptides from dried biomass and subsequent LCHRMS/MS (Section 2.6). Microcystis cells from $25 \mathrm{~mL}$ of culture were harvested on pre-weighed 
and dried (overnight at $40{ }^{\circ} \mathrm{C}$ ) glass microfibre filters (Whatman, GF/A, diameter $47 \mathrm{~mm}, \sim 1.6$ $\mu \mathrm{m}$ ) using a filtration apparatus (Millipore). After drying in an oven at $40{ }^{\circ} \mathrm{C}$ overnight, the glass microfibre filters with Microcystis cells were transferred to disposable culture tubes $(16$ x 100mm, VWR) and $14 \mathrm{~mL}$ of $80 \%$ aqueous methanol was added. For cell lysis, tubes were vortexed and sonicated for 30 seconds and stored at $-20^{\circ} \mathrm{C}$ for 1 hour. Tubes were subsequently thawed to room temperature and the freeze-thaw cycle including agitation and sonication was repeated two additional times. Resulting methanolic extracts containing intracellular cyanopeptides were filtered through $0.22 \mu \mathrm{m}$ PTFE syringe filters (ChromSpec, Inc.) into amber vials and dried under a gentle stream of nitrogen gas. Extracts of Microcystis cells were reconstituted in HPLC grade methanol, transferred to $2 \mathrm{~mL}$ amber HPLC vials (Agilent), dried under a gentle stream of nitrogen gas, and stored at $-20^{\circ} \mathrm{C}$ prior to LC-MS/MS analysis (Section 2.6).

\subsection{Cyanopeptide extraction from environmental samples.}

Investigation of the cyanopeptides within the environment for analysis of intra- and inter-lake variation was achieved with untargeted LC-HMS/MS and established metabolomic approaches (Section 2.6 and 2.7). Collection data for environmental samples studied are summarized in Table 2.3. To extract cyanopeptides from these samples, $15 \mathrm{~mL}$ of surface water was lyophilized and the resulting material was suspended in $3 \mathrm{~mL}$ of $80 \%$ aqueous methanol. The solution was sonicated and vortexed for 5 minutes each. Surface water samples concentrated on glass microfiber filter papers were processed with the freeze-thaw extraction method used for the cultivated strains. The extracts were filtered through a PTFE syringe filter into an amber vial and dried under a gentle stream of nitrogen gas. Bloom samples were filtered onto glass microfiber filter papers upon collection and processed according to Section 2.4. 
Table 2.3: Collection information for bloom and water samples collected in Ontario and Quebec.

\begin{tabular}{|c|c|c|c|}
\hline Sample Number & Type & Collection Date & Collection Site \\
\hline$(1)$ & Water & $08 / 19$ & Wildwood Conservation \\
\hline$(2)$ & Water & $08 / 19$ & Scugog Lake \\
\hline$(3)$ & Bloom & $09 / 19$ & Lac Breton \\
\hline$(4)$ & Bloom & $10 / 13$ & Lake Land \\
\hline$(5)$ & Bloom & $06 / 13$ & Lake Land \\
\hline$(6)$ & Bloom & $06 / 09$ & Lake Heney \\
\hline$(7)$ & Bloom & $07 / 13$ & Lake Land \\
\hline$(8)$ & Bloom & $06 / 10$ & Lake Land \\
\hline$(9)$ & Bloom & $06 / 18$ & Lake Land \\
\hline
\end{tabular}

\subsection{Untargeted LC-HRMS/MS analysis.}

Visualization of the entire cyanopeptide profiles were performed with untargeted LC-HRMS/MS analysis and post-data acquisition metabolomic approaches. LC-HRMS/MS analyses of resulting Microcystis extracts and environmental samples was achieved using an Agilent 1290 HPLC (Agilent Technologies) coupled to a Q-Extractive Quadrupole Orbitrap (Thermo Fisher Scientific) mass spectrometer. Chromatography conditions included an Eclipse Plus C18 RRHD (2.1 x 100mm, $1.8 \mu \mathrm{m}$; Agilent Technologies) maintained at $35^{\circ} \mathrm{C}$ and mobile phase consisting of $0.1 \%$ formic acid (mobile phase A) and acetonitrile with $0.1 \%$ formic acid (mobile phase B). The 10.5 minute gradient program optimized for cyanobacteria oligopeptide analysis increased from 0 to $35 \% \mathrm{~B}$ over $1.5 \mathrm{~min}$, increased again to $45 \% \mathrm{~B}$ over the following $2.5 \mathrm{~min}$, and finally increased to $100 \% \mathrm{~B}$ over $2 \mathrm{~min}$, where it was held at $100 \% \mathrm{~B}$ for $3 \mathrm{~min}$ prior to returning to $0 \% \mathrm{~B}$ over 0.5 min, and maintained at $0 \% \mathrm{~B}$ for $1 \mathrm{~min}$. Five $\mu \mathrm{L}$ of each intracellular and surface water extract constituted in $90 \%$ aqueous methanol was injected and resolved at a flow rate of $0.3 \mathrm{~mL} / \mathrm{min}$.

Heated electrospray ionization in positive mode was used with the following settings: capillary 155 voltage, $3.9 \mathrm{kV}$; capillary temperature, $400^{\circ} \mathrm{C}$; sheath gas, 19 units; auxiliary gas, 8 units; 
probe 156 heater temperature, $450^{\circ} \mathrm{C}$; S-Lens RF level, 45.00. MS data were acquired using untargeted DDA 157 that included a full MS scan at 70000 resolution, with a scan range of 106.7$1600 \mathrm{~m} / \mathrm{z}$; automatic 158 gain control target, $3 \times 10^{6}$; and a maximum injection time of $250 \mathrm{~ms}$. The five highest intensity ions were selected from each full scan for MS/MS analysis using a 1.0 Da isolation window, and were analyzed using the following conditions: resolution, 17 500; automatic gain control target, $1 \times 10^{6}$; max IT, $64 \mathrm{~ms}$; normalized collision energy, 37.5; intensity threshold, $1.5 \times 10^{5}$; dynamic exclusion, $5 \mathrm{~s}$.

\section{$\underline{2.7 \text { Metabolite identification. }}$}

The metabolomic processing of the large untargeted LC-HRMS/MS datasets acquired utilizes multivariate analysis (PCA, factor loading analysis) in R-software packages (xcms, MetabolAnalyze, FactoMineR), the diagnostic fragmentation filtering (DFF) module within the open-source mass spectrometry platform MZmine2 (http://mzmine.github.io/), and the Global Natural Product Social (GNPS) Molecular Networking algorithm (https://gnps.ucsd.edu/ProteoSAFe/static/gnps-splash.jsp, Section 2.9, Figure 2.2). 


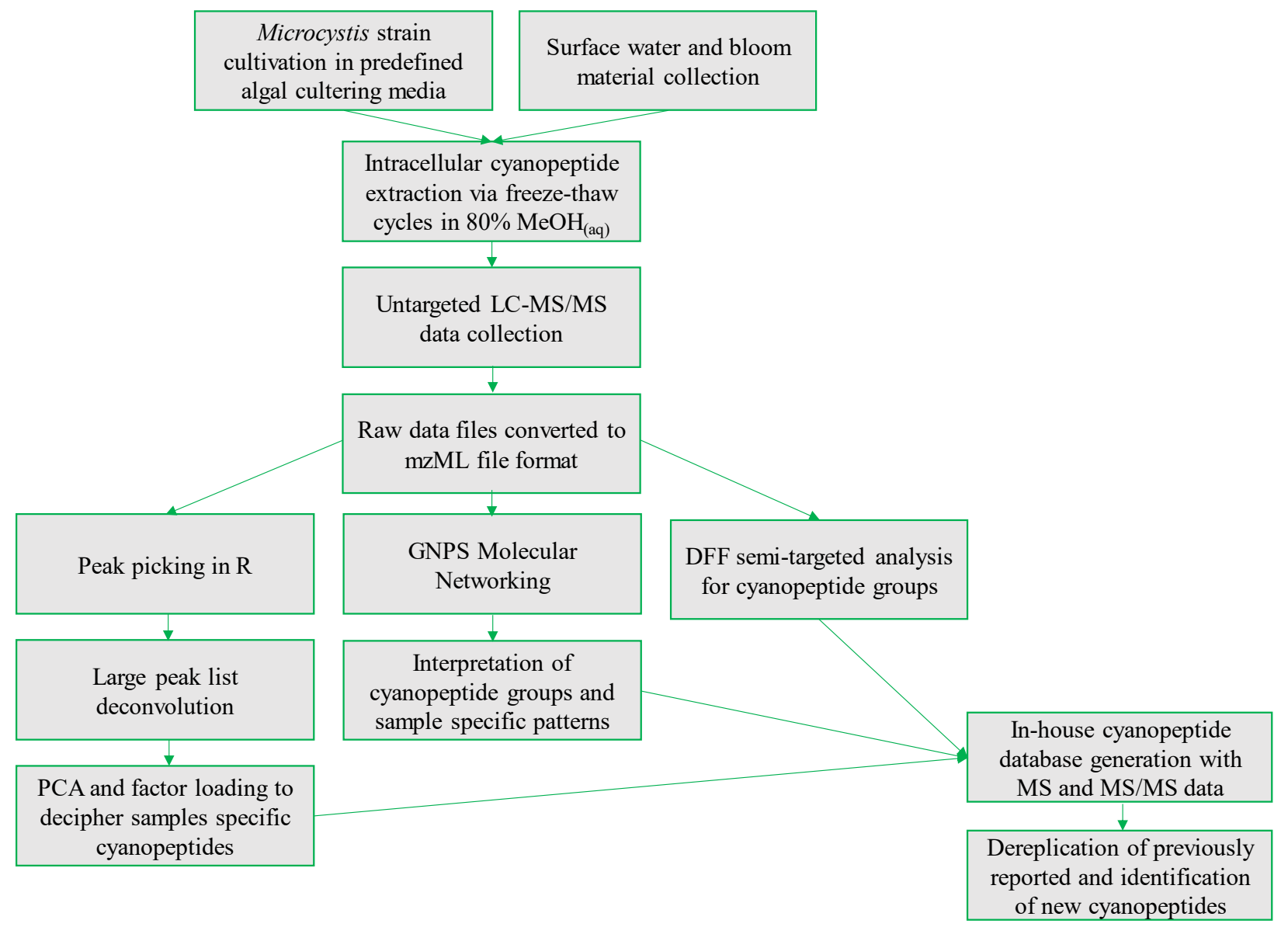

Figure 2.2: Workflow of the unique LC-MS/MS metabolomic data processing performed.

Untargeted and multivariate techniques such as PCA, factor loading, and GNPS molecular networking enable visualization of metabolome differences between strains, experimental conditions, and environmental samples through comparison and consideration of all compounds present within the sample. The multivariate analysis is an effective data mining strategy for natural product diversity, detection of novel metabolites, and to identify factors that regulate natural product biosynthesis. PCA and factor loading consider only precursor ion $\mathrm{m} / \mathrm{z}$ data with an additional statistical test within factor loading aiding in understanding of which compounds statistically contributing to sample variation whereas GNPS molecular networking utilizes similar product ion spectra to group compounds that are structurally related. MS/MS spectra contain 
characteristic product ions that are unique to the structural features for a metabolite. Structurally related compounds will generate similar product ions within the MS/MS spectra. DFF is a semitargeted approach which targets specific molecular features via diagnostic product ions to search an untargeted LC-MS/MS dataset for chemicals containing a specific molecular feature. Cyanopeptides are grouped based on the presence of unique non-proteinaceous amino acids or partial amino acid sequences which generate diagnostic product ions that can be used for cyanopeptide group identification. Some identified molecular features of cyanopeptide classes have been identified and the product ions that are generated are summarized in Table 2.4. These structural features were targeted using DFF in this study of cyanopeptide diversity.

Table 2.4: Unique structural features characteristic of different cyanotoxin groups.

\begin{tabular}{|l|l|l|}
\hline Cyanopeptide group & Structural feature(s) & Product ions $\mathbf{m} / \mathbf{z}$ \\
\hline Microcystin & Adda & $135.0803,163.1113$ \\
\hline Cyanopeptolin & Lxx-Ahp, Phe-Ahp & $181.1331,215.1167$ \\
\hline Aeruginosin & Choi & $140.1099,122.0966$ \\
\hline Microginin & Di-, mono-, non-Cl- Ahoa & $168.0338,134.0727,100.1122$ \\
& Di-, mono-, non-Cl- NMeAhoa & $182.0494,148.0883,114.1278$ \\
\hline
\end{tabular}

Cyanotoxins are identified here by predicted molecular formula from high-resolution MS data, de novo sequencing of MS/MS spectra, and spectral library and metabolite database (in-house or published) searches. This unique metabolomic workflow allows rapid dereplication of previously reported metabolites and identification of potentially novel compounds.

\section{$\underline{2.8 \text { Untargeted LC-HRMS/MS data processing. }}$}

Prior to performing multivariate analyses on the large LC-HRMS/MS data sets, elimination of mass spectral artifacts and deconvolution of peaks is required for confident reporting of compounds identified as unique to each sample. Raw MS data files were converted to centroid .mzML files with MSConvert v3.1.19 (http://proteowizard.sourceforge.net/) with peak picking set 
at an MS level of 1-2. The mzML files were subsequently processed in this file format in R 3.5 . (https://www.r-project.org/). Peak areas for metabolites within the data files were extracted using the $x \mathrm{cms}$ package with the parameters in Table 2.5 .

Table 2.5: The peak picking parameters used with the bioinformatics package, $x \mathrm{cms}$.

\begin{tabular}{|c|c|c|c|c|c|c|}
\hline Parameter & Polarity & Scan pre-filter* & $\begin{array}{c}\mathbf{m} / \mathbf{z} \\
\text { deviation } \\
\text { (ppm) }\end{array}$ & $\begin{array}{c}\text { s/n } \\
\text { ratio } \\
\text { cutoff }\end{array}$ & $\begin{array}{c}\text { Peak width } \\
\text { range (secs) }\end{array}$ & $\begin{array}{c}\text { Noise } \\
\text { level }\end{array}$ \\
\hline Set point & + & $\begin{array}{c}5 \text { with minimum } \\
\text { intensity of 5000 }\end{array}$ & 1 & 5 & $12-15$ & 500000 \\
\hline
\end{tabular}

*Number of scans a metabolite must be present in with a minimum intensity of 5000 .

Deconvolution of the extracted peak list was performed with removal of features outside the oligopeptide retention time range of 2-6.5 minutes, below $400 \mathrm{~m} / \mathrm{z}$, and if their peak area multiplied by 50 was less than the peak area of the same feature in the blank. Electrospray ionization (ESI) gives rise to adducts, which are charged molecules that can be manipulated by the mass spectrometer and generate multiple precursor ions for the one metabolite. Common adducts generated in positive ESI mode are protonated, dehydrated, sodiated, and aminated ions that correspond to the same molecule (Table 2.6). Multiple adducts for the same metabolite can occur and were removed from the peak list by identification of the characteristic mass differences and alignment of MS/MS patterns. While multiple ESI adducts for the same ionized molecular complicate the analysis of metabolomic data, they can be confirmatory when present in the same MS spectrum. Peak information was gathered into a cyanotoxin database for the metabolites detected from the five intracellular Microcystis extracts including retention time, precursor ion $m / z$, adduct $m / z, m / z$ of product ions, and which strain produced it. The in-house database identified previously characterized metabolites by comparison of high-resolution precursor ion $\mathrm{m} / \mathrm{z}$ and acknowledged cyanotoxins not previously reported (Supplemental database). 
Table 2.6: Common adducts generated by electrospray ionization in positive mode.

\begin{tabular}{|l|l|}
\hline Adduct Identity & Mass difference (Da) \\
\hline$[\mathrm{M}+\mathrm{H}]^{+}$ & +1.0018 \\
\hline$\left[\mathrm{M}+\mathrm{H}-\mathrm{H}_{2} \mathrm{O}\right]^{+}$ & -18.0102 \\
\hline$\left[\mathrm{M}+\mathrm{H}-\mathrm{SO}_{3}\right]^{+}$ & -79.9571 \\
\hline$[\mathrm{M}+\mathrm{Na}]^{+}$ & +21.9879 \\
\hline$\left[\mathrm{M}+\mathrm{NH}_{4}{ }^{+}\right]^{+}$ & +17.0253 \\
\hline
\end{tabular}

Putative structural information was generated by comparison of MS and MS/MS data and proposed fragmentation patterns of the reported structure. Chemicals that did not match literature cyanopeptide reports were designated into a chemical class based on retention time range, precursor ion $\mathrm{m} / \mathrm{z}$ range, presence of adducts, and diagnostic product ions. Variants within a chemical class were described by comparing the MS/MS to a known metabolite within the same class. Small chemical modifications and amino acid substitutions were characterized with this method as well as comparison of the precursor ion $\mathrm{m} / \mathrm{z}$ and molecular formula. This MS/MS based approach cannot discriminate residue configurations, nor isobaric residues. For example, Lxx is used to annotate either leucine (Leu) or isoleucine (Ile) except when reporting a known compound.

\subsection{Post-Data Acquisition Processing and Visualization.}

\subsubsection{Principal component analysis and factor loading analysis.}

The following multivariate analyses allow for interpretative of the large MS dataset generated and provides significance as to which features (compounds) statistically contribute to the variance between samples. The $\log _{10}$ of the processed peak areas (Section 2.8) were utilized in $\mathrm{R}$ for principal component analysis and generation of a loading plot using the packages MetabolAnalyze and FactoMineR. All dimensions of the PCA were analyzed, with dimensions 1 and 4 showing the best separation and thus variation explained between the 5 studied strains. The triplicate data was 
then grouped and a Kruskall-Wallis test with Benjamini-Hochberg correction was performed to investigate the statistical significance of the features and their contribution to the PCA. Features with a p-value $<0.05$ were grouped and labelled red within the loading plot. To demonstrate which metabolites contributed to the variation, the loading and PCA data was exported to excel and previously identified metabolites were identified by $\mathrm{m} / \mathrm{z}$ and retention time and identified by numbers within the loading plot.

\subsubsection{Heat map analysis to depict media effects on cyanopeptide production.}

Metabolites previously identified from the molecular network were selected for heat map analysis for determination of media effects on cyanopeptide production and production as a function of time. Peak areas were extracted from data files corresponding to day 5, 10, 15, 20, and 25 intracellular extracts of M. aeruginosa CPCC 300, СРCC 632 and CPCC 633, and M. flos-aquae CPCC 461 when grown in the two defined culture media BG-11 and MA. Peak areas for the

selected cyanopeptides were normalized with dried biomass and excluded if it did not exceed $5 \mathrm{E}^{+04}$ at any time point. Heat maps were generated with $\log _{10}$ normalized peak area per strain, per media, in $\mathrm{R}$ using the following packages: gplots for heatmap generation and ColorRamps for visualization.

\subsubsection{GNPS Molecular networking analysis.}

The mzML files corresponding to both the intracellular extracts of the five studied Microcystis strains and a methanol blank were uploaded to the GNPS network (https://gnps.ucsd.edu/ProteoSAFe/static/gnps-splash.jsp) with FileZilla (https://filezillaproject.org/) through the ccms host. A molecular network that groups metabolites by structurally 
similarity based on high-resolution MS/MS data was generated with a precursor ion $\mathrm{m} / \mathrm{z}$ tolerance of 0.02 and a product ion $m / z$ tolerance of 0.03 . A minimum of five matching fragment ions and cluster size equal to or greater than two was required for grouping. The graphML file format from the GNPS website was uploaded into cytoscape for visualization (https://cytoscape.org/). The blank data file was used for data deconvolution with all metabolites present in the blank deleted from the network. Further deconvolution included the elimination of metabolites outside the retention time range 2-6.5 minutes, below $400 \mathrm{Da}$, and any remaining single nodes. Nodes were coloured based on which data file they were present in as well as outlined corresponding to their charge (singly, doubly, or triply).

\subsubsection{Diagnostic fragmentation filtering (DFF) for cyanopeptide group detection.}

The mzML files were uploaded into MZmine v.2.38 (http://mzmine.github.io/) and a diagnostic fragmentation filtration plot was generated for the detection of all compounds within the intracellular extract containing diagnostic molecular features characteristic of the studied chemical groups (Table 2.4). The diagnostic product ions $\mathrm{m} / \mathrm{z} 134.0962$ and 181.1325 were used for cyanopeptolins containing the sequence NMePhe-Leu-Ahp with a minimum base peak intensity of $10 \%$. For detection of cyanopeptolins containing the sequence NMeTyr-Phe-Ahp, the product ions $\mathrm{m} / \mathrm{z} 150.0917$ and 215.1175 were used with a minimum diagnostic ion intensity of $10 \%$. For detection of all cyanopeptolins within environmental samples, the four possible sequences were utilized (NMePhe-Leu-Ahp, NMe-Phe-Phe-Ahp, NMe-Tyr-Leu-Ahp, and NMe-Tyr-Phe-Ahp). All DFF plots generated had an $\mathrm{m} / \mathrm{z}$ tolerance of $0.02 \mathrm{~m} / \mathrm{z}$ or $5.0 \mathrm{ppm}$. Similar analyses were performed for the detection of variants within the microcystin, aeruginosin, and microginin chemical groups. 


\section{$\underline{\text { 3. Results }}$}

\subsection{Untargeted mass-spectrometry based metabolomics: application to cyanopeptide groups}

\subsubsection{Cyanopeptide identification}

Microcystis species are known to biosynthesize structurally diverse mixtures of peptide natural products (cyanopeptides; (Tiam et al., 2019). Untargeted MS metabolomics allows comparison and differentiation of the complete chemical profiles for the five investigated Microcystis strains. The strains were grown in $\mathrm{BG}-11$ media at $25^{\circ} \mathrm{C}$ for 15 days and the intracellular metabolites were extracted with $80 \%$ aqueous methanol. The intracellular cyanopeptides that are the focus of this thesis are peptide natural products and are grouped based on the presence of characteristic structural features, non-proteinaceous amino acids, or partial amino acid sequences. These unique structural features generate similar product ions within the MS/MS of structurally related metabolites. We compiled characteristic MS and MS/MS data for each cyanopeptide group including precursor ion ranges, retention time ranges, and product ion spectra patterns to distinguish metabolites in the different cyanopeptide groups (microcystins, cyanopeptolins, anabaenopeptins, microginins, aeruginosins, cyanobactins, and microviridins) within the untargeted MS data processing techniques such as molecular networking, principal component analysis, and factor loading.

\subsubsection{Principal component analysis of LC-HRMS data}

Untargeted metabolomic strategies such as principal component analysis (PCA), factor loadings plot, and GNPS molecular networking (https://gnps.ucsd.edu/ProteoSAFe/static/gnps-splash.jsp) were utilized to describe the strain specific cyanopeptide diversity from the five Microcystis strains studied. With large high-resolution MS data sets, metabolomics approaches are useful for 
interpretation, statistical analysis, and visualization of the metabolome. PCA works to describe the variation between complex samples using a collection of variables that defines each sample. To understand the chemical variation of the five Microcystis strains, 262 metabolites detected with the $x c m s$ package are the variables used to assess similarities and differences in the chemical profiles (peak picking parameters described in materials and methods). The $\log _{10}$ peak area for the selected 262 features were analyzed with the packages MetabolAnalyze and FactoMineR in the R programming environment to generate a PCA (Figure 3.1) and a factor loading plot (Figure 3.2). The factor loading plot pairs with the PCA and provides insight into which chemical variables contribute to the variance and resemblance of the metabolomes for the investigated Microcystis cultures. 


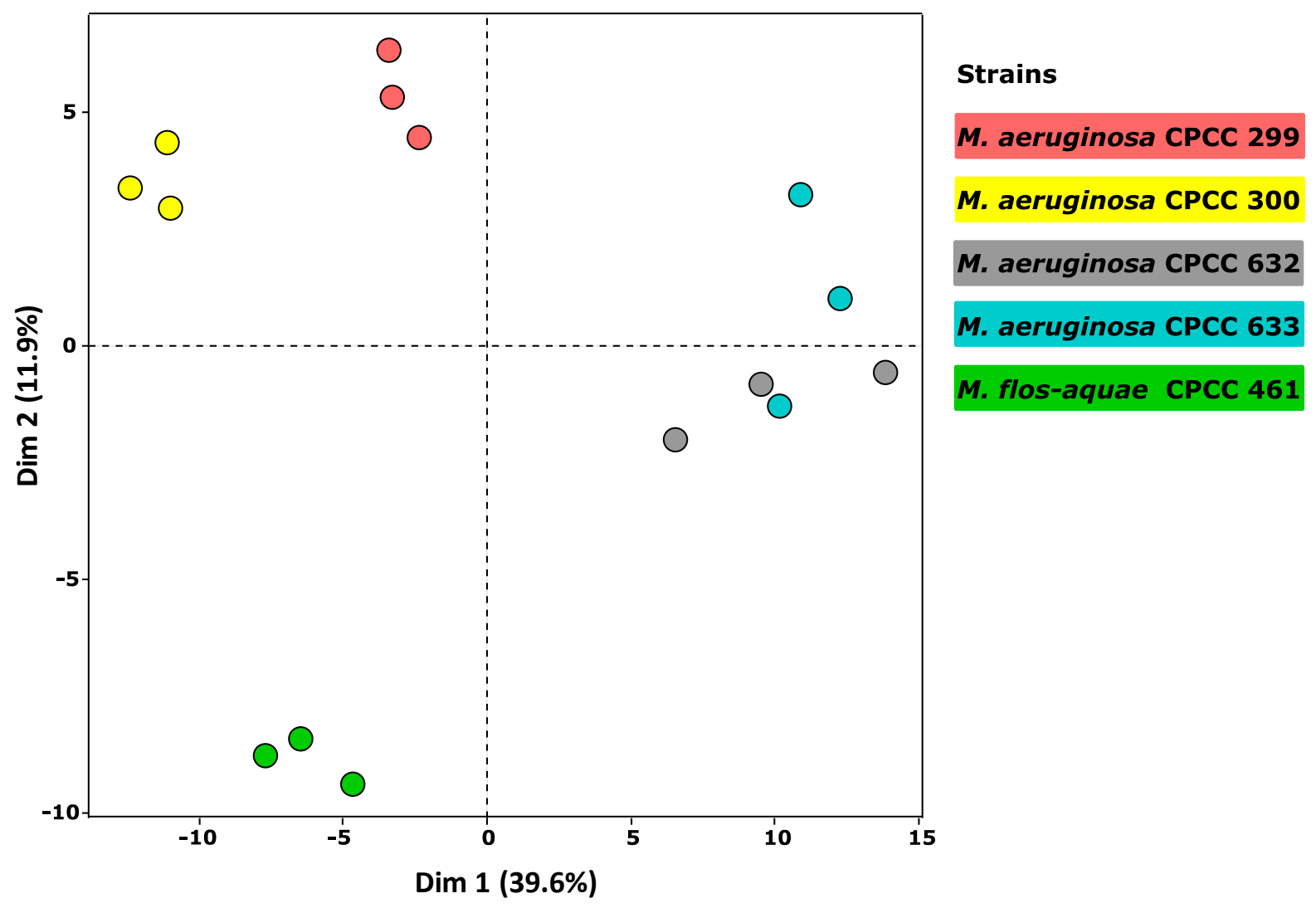

Figure 3.1: Principal component analysis (PCA) of triplicate Microcystis aeruginosa CPCC 299, CPCC 300, CPCC 632, and CPCC 633, and M. flos-aquae CPCC 461 cultures grown in BG-11 medium.

In the present study, untargeted MS metabolomic approaches were used to describe the intracellular cyanopeptide diversity from four Microcystis aeruginosa (CPCC 299, CPCC 300, CPCC 632, CPCC 633) and one M. flos-aquae strains (CPCC 461, Table S1). A total of 262 features were detected using the $x c m s$ bioinformatics package (search parameters are described in the materials and methods). A PCA plot was constructed with these features to visualize the metabolomic variation between the strains based on cyanopeptide identities and their relative abundances (Figure 3.1). The PCA plot displayed four groups revealing distinct differences between the studied Microcystis strains cyanopeptide profiles. The majority of variation is 
described with dimension 1 (39.6\%) which groups M. aeruginosa CPCC 632 (blue) and CPCC 633 (grey) on the positive side of the y-axis and grouping of M. aeruginosa CPCC 299 (red), CPCC 300 (yellow), and M. flos.aquae CPCC 461 (green) on the negative side of the y-axis. Further description of the variation occurs with the addition of dimension $2(11.9 \%)$, which provides minor separation of the triplicate data points for M. aeruginosa CPCC 632 and CPCC 633 but large separation of M. flos-aquae CPCC 461 from its grouping in dimension 1 with M. aeruginosa CPCC 299 and CPCC 300. M. aeruginosa CPCC 299 and CPCC 300 group within the top left quadrant of the PCA plot suggesting similarity in their intracellular metabolomes. These two strains are known microcystin producers (i.e., toxic strains), whereas the other investigated Microcystis strains are considered non-toxic (Racine et al., 2019). Differences in the chemical profiles for the two toxic strains are implied because of the slight separation of the set of triplicate data points. The metabolomic profile of M. flos-aquae CPCC 461 was vastly different compared to the $M$. aeruginosa strains, appearing alone in the bottom left quadrant of the PCA plot. Grouping of this strain within dimension 1 with the two toxic strains M. aeruginosa CPCC 299 and CPCC 300 suggests some metabolome similarity between these strains. M. aeruginosa CPCC 632 and CPCC 633 overlap in the PCA plot when considering both dimensions, indicating strong similarity in their cyanopeptide profiles. The specific metabolites and metabolite groups that differentiate the Microcystis strains were elucidated through investigation of a factor loadings plot, which visualizes the variables that dictate the spatial separation of the individuals within the PCA.

Further investigation into the features that significantly differentiate the strains was performed with a factor loading plot containing 262 variables. Using the dplyr package in R, a Kruskal-Wallis test for non-parametric data with Benjamini-Hochberg correction was performed to determine which metabolites were statistically contributing to the chemical variation of these strains $(p<$ 
0.05). 140 metabolites were deemed statistically significant when comparing the chemical profiles of the five Microcystis strains and are highlighted with red arrows (Figure 3.2). The coordinate location where the arrow lands within this plot is specific and determined by which strain produces it. Cyanopeptides identified based on HR-MS, retention time, fragmentation patterns and database searching have been annotated within the feature loadings plot to highlight the diversity of cyanopeptides and cyanopeptide groups produced by each Microcystis strain.

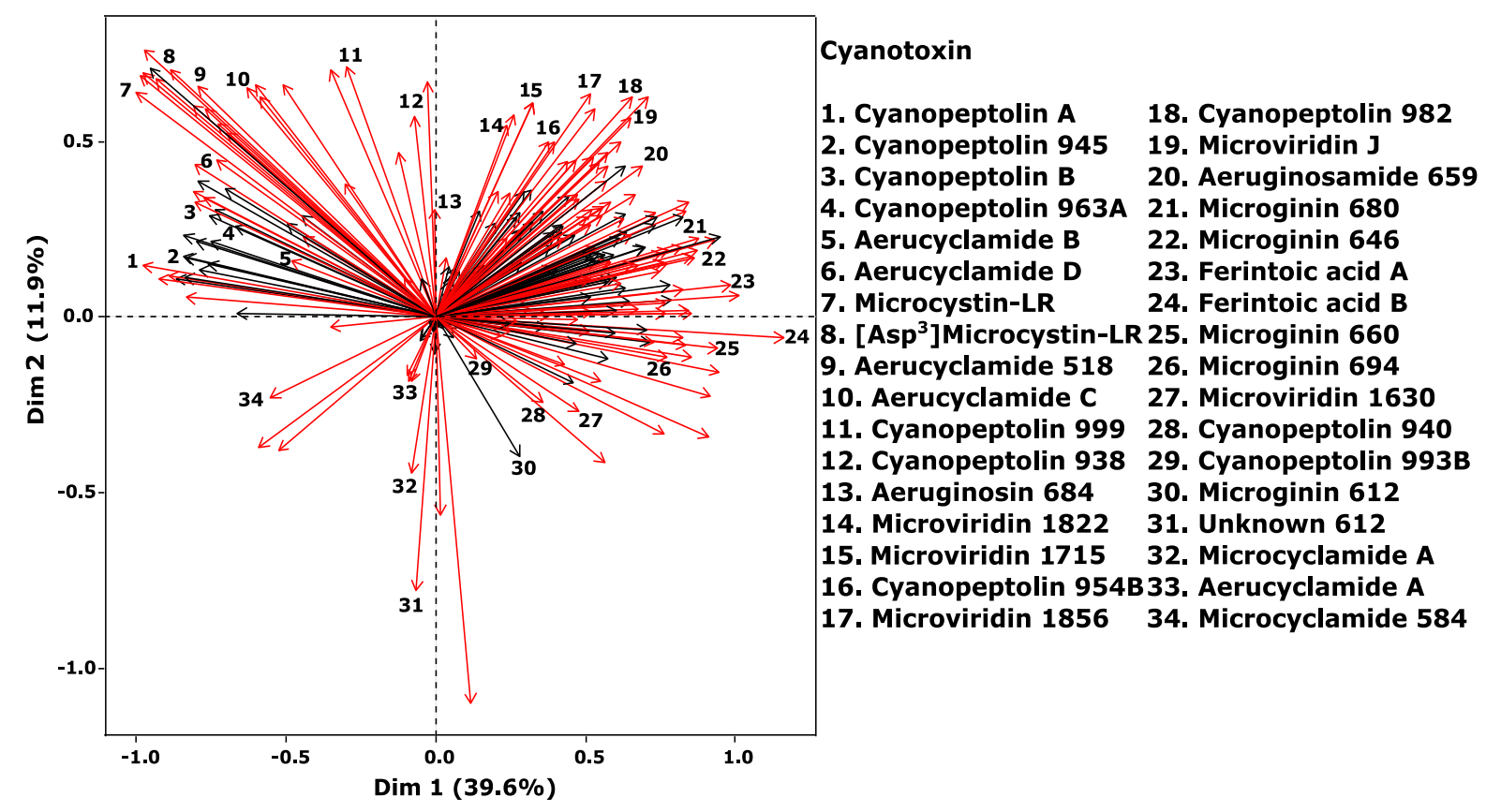

Figure 3.2: The loading plot contains all factor loadings considered in the PCA. Of 787 total variables, 262 are considered in the PCA and visualized in the loading plot. Factors included fall within the precursor ion range $m / z 400-1200$ (unless present within the molecular network) to target oligopeptide cyanopeptides, 2.0-6.5 min retention time range, and above a peak threshold of $5 \mathrm{E}^{+06}$. Red arrows indicate statistical significance in contribution to the individual strains in the PCA ( $p<0.05$ Kruskal-Wallis test with Benjamin-Hochberg correction). Specific cyanopeptides identified by their precursor ion $m / z$, MS/MS data and retention time are numbered.

When analyzing the statistically significant features (red) within the loading plot (Figure 3.2), the identity of MC-LR $\left(\mathrm{m} / \mathrm{z} 995.5565[\mathrm{M}+\mathrm{H}]^{+} ; 7\right.$ and $\left[\mathrm{Asp}^{3}\right]-\mathrm{MC}-\mathrm{LR}\left(\mathrm{m} / \mathrm{z} 981.5391[\mathrm{M}+\mathrm{H}]^{+} ; 8\right)$ were confirmed by comparison to purified reference materials (NRC Halifax) and agree with the 
literature, which states M. aeruginosa CPCC 299 and CPCC 300 produce these congeners (Racine et al., 2019). These MC features statistically contribute to the similarity of these strains chemical profiles as well as in differentiating them from the other investigated strains.

Other features within the loading that are significant to the M. aeruginosa CPCC 299 and CPCC 300 chemotypes, in a similar manner as the MCs, are aerucyclamide 518 , aerucyclamide $\mathrm{C}$, and aerucyclamide D $\left(m / z 519.2382[\mathrm{M}+\mathrm{H}]^{+} ; 9, m / z 517.2232[\mathrm{M}+\mathrm{H}]^{+} ; 10, m / z 587.1567[\mathrm{M}+\mathrm{H}]^{+} ; 6\right.$, (Portmann, Blom, Kaiser, et al., 2008)). Features distinguishing M. aeruginosa CPCC 300 are four cyanopeptolin congeners, cyanopeptolin A, cyanopeptolin B, cyanopeptolin 963A, and cyanopeptolin $945\left(957.5393[\mathrm{M}+\mathrm{H}]^{+} ; 1,929.5072[\mathrm{M}+\mathrm{H}]^{+} ; 3,964.5032[\mathrm{M}+\mathrm{H}]^{+} ; 4,946.4899\right.$ $\left.[\mathrm{M}+\mathrm{H}]^{+} ; 2\right)$. The first three listed congeners have been previously reported from M. aeruginosa and have the partial amino acid sequence NMe-Phe-Lxx-Ahp (Bister et al., 2004; Cornel et al., 1993). Distinguishing metabolites for M. aeruginosa CPCC 299 are cyanopeptolin 977 and cyanopeptolin $938\left(\mathrm{~m} / z 978.4592[\mathrm{M}+\mathrm{H}]^{+} ; 11, \mathrm{~m} / z 939.4628[\mathrm{M}+\mathrm{H}]^{+} ; 12\right)$. These two cyanopeptolins have not been previously reported in the literature. Investigation of their MS/MS patterns revealed they have the partial amino acid sequence NMe-Phe-Phe-Ahp by comparison to the MS/MS of the known metabolite Micropeptin 996 with this partial amino acid sequence (Strangman et al., 2018). Other cyanopeptolin features with the partial amino acid sequence NMe-Phe-Phe-Ahp identified from the factor loading analysis specific for the M. aeruginosa CPCC 299 and CPCC 632 chemotypes cyanopeptolin 954B $\left(\mathrm{m} / \mathrm{z} 955.4263[\mathrm{M}+\mathrm{H}]^{+} ; 16\right)$, and cyanopeptolin $982(\mathrm{~m} / \mathrm{z}$ $\left.983.4639[\mathrm{M}+\mathrm{H}]^{+} ; 18\right)$. Also shared by these two strains are microviridin congeners, microviridin $\mathrm{J}\left(\mathrm{m} / \mathrm{z} 842.8925[\mathrm{M}+2 \mathrm{H}]^{2+} ; 19\right)$, microviridin $1822\left(\mathrm{~m} / \mathrm{z} 912.4276[\mathrm{M}+2 \mathrm{H}]^{2+} ; 14\right)$, microviridin $1715\left(\mathrm{~m} / \mathrm{z} 858.9321[\mathrm{M}+2 \mathrm{H}]^{2+} ; 15\right)$, and microviridin $1856\left(\mathrm{~m} / \mathrm{z} 922.3999[\mathrm{M}+2 \mathrm{H}]^{2+} ; 17\right.$, (Rohrlack et al., 2003)). The production of similar congeners within both the cyanopeptolin and microviridin 
chemical group show overlapping biosynthetic potential by M. aeruginosa CPCC 299 and CPCC 632.

The PCA plot (Figure 3.1) shows that M. aeruginosa CPCC 632 and CPCC 633 have similar cyanopeptide profiles. Both strains produce the anabaenopeptins, ferintoic acid A and ferintoic acid B $\left(m / z 867.4378[\mathrm{M}+\mathrm{H}]^{+} ; 23, m / z 881.4552[\mathrm{M}+\mathrm{H}]^{+} ; 24\right.$, (Williams et al., 1996)). The distinct features that distinguish the chemotypes for M. aeruginosa CPCC 632 and CPCC 633 are congeners within the groups; cyanopeptolins, microginins, and microviridins, which are known to be produced by Microcystis species (Welker et al., 2006). Factor loading analysis showed that $M$. aeruginosa CPCC 632 exclusively produces the previously reported microginin 680 and microginin $646\left(\mathrm{~m} / z 681.2441[\mathrm{M}+\mathrm{H}]^{+} ; 21, \mathrm{~m} / z 647.2826[\mathrm{M}+\mathrm{H}]^{+} ; 22\right.$, (Strangman \& Wright, 2016)), whereas M. aeruginosa CPCC 633 exclusively produces microginin $612(\mathrm{~m} / z 613.3225$ $[\mathrm{M}+\mathrm{H}]^{+} ; 30,($ Strangman \& Wright, 2016)). These two strains overlap in the production of the not previously documented microginins 660 and $694\left(\mathrm{~m} / z 661.2985[\mathrm{M}+\mathrm{H}]^{+} ; 25\right.$, and $\mathrm{m} / z 695.2599$ $\left.[\mathrm{M}+\mathrm{H}]^{+} ; 26\right)$. Variation arises in the exclusive production of cyanopeptolin congeners with different partial amino acid sequences. M. aeruginosa CPCC 633 produces cyanopeptolin 940 and cyanopeptolin 992 with the partial amino acid sequence NMe-Cl-Tyr-Phe-Ahp and NMeTyr-PheAhp, respectively $\left(m / z 923.4039\left[\mathrm{M}+\mathrm{H}-\mathrm{H}_{2} \mathrm{O}\right]^{+} ; 28, m / z 979.4930\left[\mathrm{M}+\mathrm{H}-\mathrm{H}_{2} \mathrm{O}\right]^{+} ; 29\right)$ and $M$. aeruginosa CPCC 632 produces cyanopeptolin 954B and cyanopeptolin 982 with the partial amino acid sequence $\left(m / z 955.4263[\mathrm{M}+\mathrm{H}]^{+} ; 16, m / z 983.4639[\mathrm{M}+\mathrm{H}]^{+} ; 18\right)$. Like M. aeruginosa $\mathrm{CPCC}$ 299 and CPCC 632, CPCC 633 also has the biosynthetic machinery to synthesize microviridin congeners with exclusive production of the congener, microviridin $1630\left(\mathrm{~m} / \mathrm{z} 816.4052[\mathrm{M}+2 \mathrm{H}]^{2+}\right.$; 27). 
M. flos-aquae CPCC 461 produced few metabolites under the growth conditions in comparison to the $M$. aeruginosa strains. There are few NRPS natural products known from $M$. flos-aquae (Urrutia-Cordero et al., 2013). The major metabolite that is unique to this strain and is highlighted within the loading plot is microcyclamide A $\left(\mathrm{m} / \mathrm{z} 583.1902[\mathrm{M}+\mathrm{H}]^{+}\right.$; 32, (Portmann, Blom, Kaiser, et al., 2008)) and to a lesser extent, a related microcyclamide metabolite $(\mathrm{m} / \mathrm{z} 585.2058$ $[\mathrm{M}+\mathrm{H}]^{+}$;34). Aerucyclamide A is also produced by M. aeruginosa CPCC 299 and CPCC 300, as well as M. flos-aquae CPCC $461(\mathrm{~m} / z \text { 517.2235 [M+H] }]^{+}$;33, (Portmann, Blom, Gademann, et al., 2008)) supporting the grouping of these strains within dimension 1 of the PCA analysis.

Chemotype information for each Microcystis strain has been elucidated via the multivariate analysis of intracellular cyanopeptide extracts. Unique co-production of the different cyanopeptide groups by the Microcystis strains proves that each isolate has a strain-specific chemotype. Loading plot analysis identified features (compounds) that statistically contributed to the variance in chemical profiles of the five Microcystis strains. Annotation of previously reported cyanopeptides was possible by HR-MS data and product ion spectra, but more in-depth analysis is required to further decipher the complex and individualistic cyanopeptide profiles of the Microcystis strains.

\subsubsection{Molecular network analysis of LC-HRMS data}

GNPS molecular networking is a technique that organizes metabolites based on their MS/MS fragmentation patterns (Tiam et al., 2019). The basis of this tool uses the premise that structurally related metabolites will generate similar product ions and product ion patterns, which can be exploited for the clustering and identification of like compounds. The GNPS algorithm converts each fragmentation spectrum into a vector with normalized product ion intensity for all metabolites to be compared. A cosine function with two vector inputs provide a numerical value of similarity for the two considered metabolites and clustering is visualized within Cytoscape with a node for 
each precursor ion and an edge demonstrating a cosine similarity score above the set threshold. The resulting network for the five Microcystis strains has 126 nodes representing their cyanopeptides and 232 edges representing the structural similarity between metabolites (Figure 3.3). With nineteen clusters that have more than two nodes, thirteen of the clusters have been categorized by their identified respective chemical group. Fourteen of the twenty-seven clusters (\#14-\#27) were not able to be identified. Annotation of chemical groups was performed based on identification of individual metabolites within the cluster by HR-MS, retention time, diagnostic product ions, and matches with library or database searches. Of the 126 metabolites, twenty-four were matched with previously reported metabolites. Each annotated group will be described in detail below. 


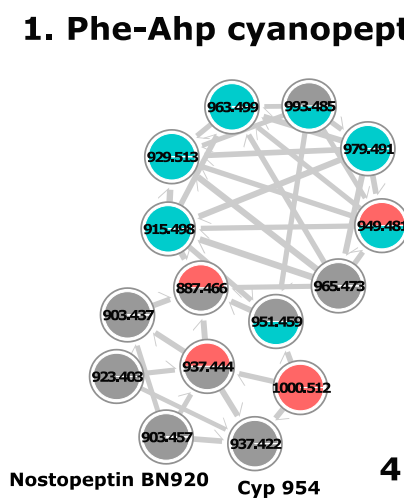

4. microviridins

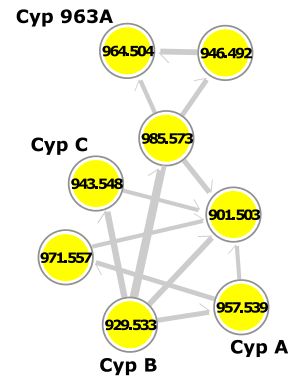

5. microviridins
M. aeruginosa CPCC 299

M. aeruginosa CPCC 300

M. aeruginosa CPCC 632

M. aeruginosa CPCC 633

M. flos-aquae CPCC 461

Singly charged

Doubly charged

Triply charged

\section{6. microcystins}

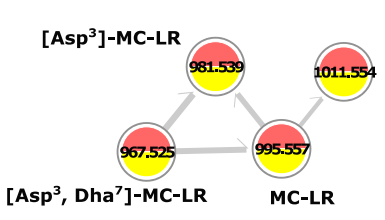

$\left[\right.$ Asp $^{3}$, Dha $\left.^{7}\right]-M C-L R \quad$ MC-LR

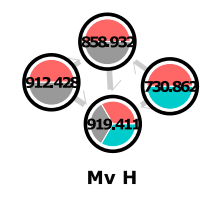

9. anabaenopeptins

$10 . \quad 11$. aeruginosins

8. aeruginosamides

\section{AP 857}
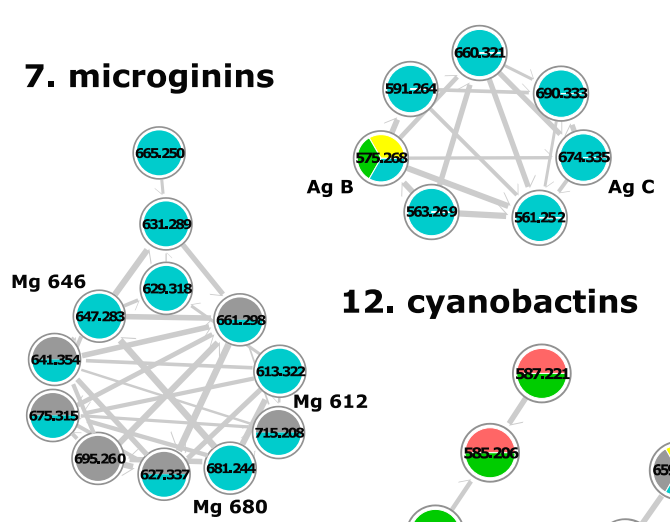

12. cyanobactins

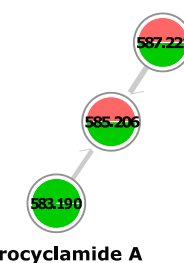

18. 19.20.

21.

22.
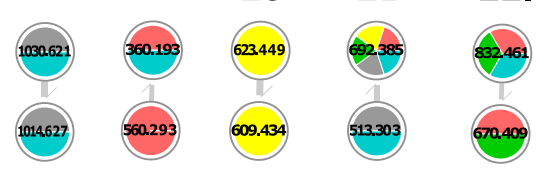

Ferintoic acid $A$

Ferintoic acid B AP D

13. cyanobactins

14.

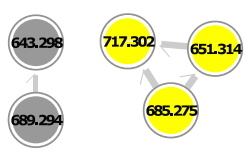

16.

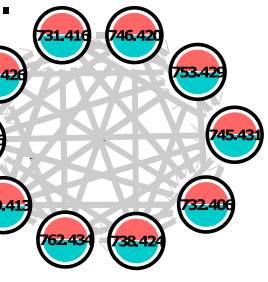

15.

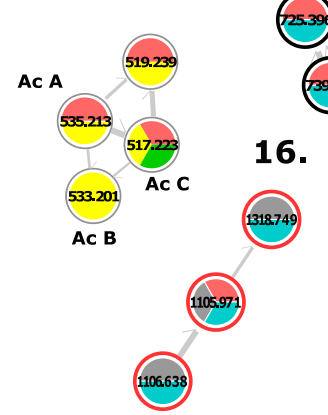

26.

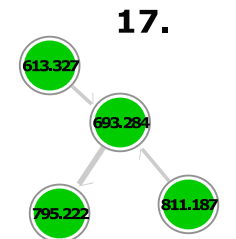

23. 24.25.
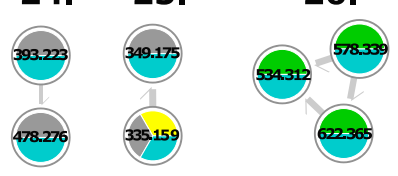

27.

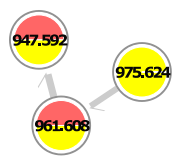

Figure 3.3: GNPS molecular network generated from metabolite MS/MS spectra produced by the five studied Microcystis strains grown in BG-11 medium. Nodes are color coded pi-charts labelled with precursor ion $\mathrm{m} / \mathrm{z}$ illustrating which strains produced each metabolite (qualitative). The width of lines connecting nodes are proportional to the corresponding cosine score. Precursor and product ion $\mathrm{m} / \mathrm{z}$ tolerance were set to 0.03 and $0.02 \mathrm{Da}$, respectively. The minimum base peak intensity was set at $5 \mathrm{E}^{+06}$. Clustering parameters required a minimum cosine similarity score of 0.6 and five matching product ions. Deconvolution was performed by eliminating all metabolites 
present within the blank, outside the retention time range 2-6.5 minutes, and clusters with a size less than 2. Cyanopeptide groups and specific metabolites identified using the GNPS spectral library, interpretation of MS/MS spectra and comparisons to the literature are labelled. Abbreviations used in this figure include Ac; aerucyclamide, Ag; aeruginosamide, Ahp; 3-amino6-hydroxy-2-piperidone, AP; anabaenopeptin, Cyp; cyanopeptolin, Lxx; leucine or isoleucine, $\mathrm{MC}$; microcystin, Md; microcyclamide, Mg; microginins and Mv; microviridin.

The chemical diversity for the five studied Microcystis strains is illustrated in the molecular network (Figure 3.3). Together, the PCA and molecular networking demonstrate extensive strain specific cyanopeptide profiles. No two strains produce the same mixture of chemical groups or congeners within the groups. M. aeruginosa CPCC 299 and CPCC 300 are considered "toxic" strains because they both produce MCs. However, they produce many other chemical groups with limited information regarding their toxicity, structures, environmental concentrations and beneficial properties. M. aeruginosa CPCC 632 and CPCC 633 also overlap with the production of similar chemical groups including microginins, anabaenopeptins, and microviridins. Again, there is very little to no information in the literature regarding the detected congeners.

Chemical variation of cyanopeptides occurs through amino acid substitutions and posttranslational modifications of the molecule, resulting in numerous congeners for each chemical class. The cyanopeptides are grouped based on unique structural features, such as nonproteinaceous amino acids or partial amino acid sequences that result in distinct product ion patterns within the MS/MS spectra of chemicals within the same group. We can exploit this consistency in chemical structures through post acquisition metabolomics techniques for the detection and identification of related structurally related metabolites.

\subsection{3a Molecular network analysis: Microcystins}


Most cyanopeptide studies focus on microcystins (MCs). Collectively, MCs are group of more than 300 potent hepatotoxins and possible human carcinogens (IARC Group 2B; Bouaïcha et al. 2019. MCs all contain the non-proteinaceous beta amino acid Adda (3-Amino-9-methoxy-2,6,8trimethyl-10-phenyldeca-4,6-dienoic acid), and in most cases possess Mdha (methyldehydroalanine). Within the MS/MS spectra of MCs, diagnostic product ions that for these diagnostic structural features are $\mathrm{m} / \mathrm{z} 135.0808$ and $\mathrm{m} / \mathrm{z} 163.1118$ of the Adda moeity and $\mathrm{m} / \mathrm{z}$ 213.0865 for Glu-Mdha (McMullin et al., 2019). Using metabolomic approaches to target these characteristic product ions provides a rapid mechanism to identify all MCs from samples. $M$. aeruginosa CPCC 299 and CPCC 300 are considered "toxic" and produce the same mixtures of MCs according to the molecular network cluster \#6, agreeing with previous metabolome reports for these strains (Figure 3.4; Racine et al., 2019). No other studied strains produced MCs. $M$. aeruginosa CPCC 300 and M. aeruginosa PCC 7806, the most studied Microcystis strain to date, have very similar chemical profiles, with overlapping production of not only MC congeners, MCLR and $\left[\mathrm{Asp}^{3}\right]-\mathrm{MC}-\mathrm{LR}$ but other specific variants within other chemical classes such as cyanopeptolins, cyanobactins, and aeruginosins (Cornel et al., 1993; Portmann, Blom, Gademann, et al., 2008). 


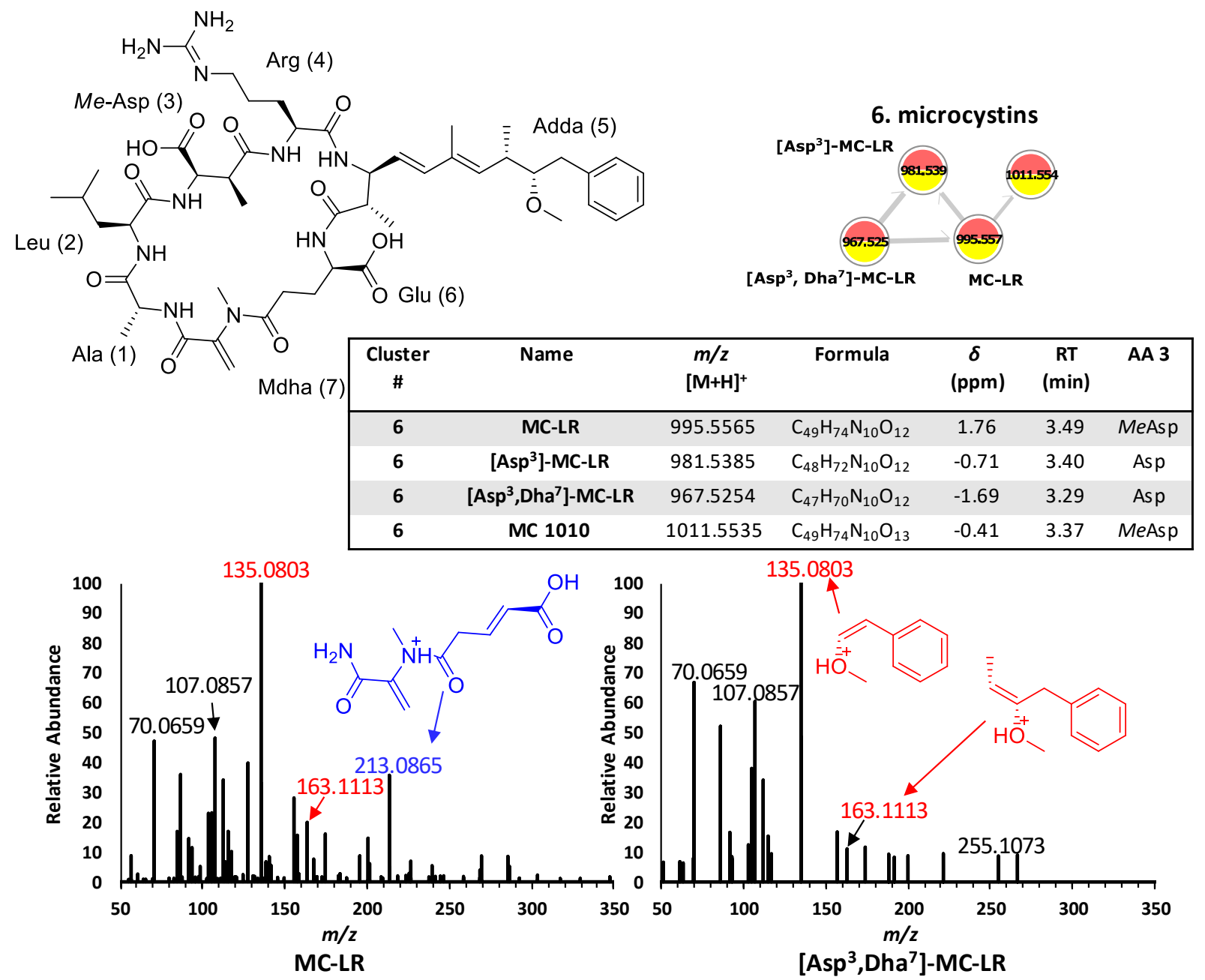

Figure 3.4: Representative chemical structure of the MC group, MC-LR (top left). The MC GNPS molecular network cluster \# 6 (top right) showing congeners produced by $M$. aeruginosa CPCC 299 (red) and CPCC 300 (yellow) with summary table of MS information for the detected MC congeners. The MS/MS of MC-LR (bottom left) and [ $\mathrm{Asp}^{3}$, Dha ${ }^{7}$-MC-LR (bottom right) with diagnostic product ions and their putative structures, $\mathrm{m} / \mathrm{z} 135.0803$ and $\mathrm{m} / \mathrm{z} 163.1113$ of the Adda moiety, and $m / z 213.0865$ of the Mdha.

Three of the four MCs within the molecular network have been previously reported, MC-LR, $\left[\mathrm{Asp}^{3}\right]-\mathrm{MC}-\mathrm{LR}$, and $\left[\mathrm{Asp}^{3}, \mathrm{Dha}^{7}\right]-\mathrm{MC}-\mathrm{LR}$ (Hollingdale et al., 2015). The identity of MC-LR and $\left[\mathrm{Asp}^{3}\right]$-MC-LR were confirmed by retention time and HR-MS in comparison to a purified standard (NRC, Halifax). For determining the identity of the MC congener $\left(\mathrm{m} / \mathrm{z} 967.5254[\mathrm{M}+\mathrm{H}]^{+}\right)$, it was considered that it is 14.0131 mass units less than [Asp $\left.{ }^{3}\right]-M C-L R$, suggesting a second demethylation. When comparing the MS/MS of MC-LR and $\left[\mathrm{Asp}^{3}\right.$, Dha $\left.{ }^{7}\right]-\mathrm{MC}-\mathrm{LR}$, the loss of the 
product ion $\mathrm{m} / \mathrm{z} 213.0865$ suggests desmethyl-dehydroalanine in position 7 for this congener (Figure 3.4). The fourth MC within the cluster does not match any congener reported in the MC database suggesting a potential new MC. This congener is 15.9970 mass units greater than MCLR suggesting hydroxylation of the molecule which could not be elucidated by product ions.

\section{$\underline{\text { 3.1.3b Molecular network analysis: Cyanopeptolins }}$}

The cyanopeptolin cyanopeptide group is distinguished by the presence of a 3-amino-6-hydroxy2-piperidone (Ahp) residue at position 2 of the hexacyclic moeity. The amino acid adjacent to the diagnostic Ahp group aids in further subclassification of cyanopeptolin congeners because the substitution remains relatively consistent between congeners within the subclass. Subclassification is required for cyanopeptolins as there is an immense number of metabolites that contain an Ahp moiety resulting in few common product ions because of the increased structural diversity. Determination of the partial amino acid sequence is done with identification of diagnostic product ions, each of which contain the Ahp moiety and the adjacent amino acid (position 3). For example, cyanopeptolin A is classified as a Lxx-Ahp cyanopeptolin because it contains a Leu in position 3 adjacent to the diagnostic Ahp moiety at position 2 and results in the following diagnostic product ions; $m / z$ 209.1283 [Leu-Ahp- $\left.\mathrm{H}_{2} \mathrm{O}+\mathrm{H}\right]^{+}$and $m / z$ 181.1331 [Leu-Ahp- $\left.\mathrm{H}_{2} \mathrm{O}-\mathrm{CO}+\mathrm{H}\right]^{+}(\mathrm{McD}$ (Lnald et al., 2020)). When identifying cyanopeptide partial sequences by MS/MS approaches, the identity of leucine and isoleucine cannot be determined because they are isobaric, and thus annotated as Lxx. Further exploring this cyanopeptide group, cyanopeptolin 954 is a Phe-Ahp cyanopeptolin as it contains a Phe residue at position 3 neighbouring the Ahp moiety resulting in the following diagnostic product ions; $m / z 243.1121$ [Phe-Ahp $\left.-\mathrm{H}_{2} \mathrm{O}+\mathrm{H}\right]^{+}$and $m / z 215.1174$ [PheAhp $\left.-\mathrm{H}_{2} \mathrm{O}-\mathrm{CO}+\mathrm{H}\right]^{+}$(Figure 6). The subclassification of cyanopeptolin congeners is illustrated with two clusters annotated as cyanopeptolins within the molecular network, cluster \#1 (Phe-Ahp) 
and \#2 (Lxx-Ahp, Figure 3.3). The substitution of the amino acid at position 3 results in large enough product ion diversity that it generates two separate network clusters representative of the subclasses of cyanopeptolins (Figure 3.5). Increased structural and sequence information arises with the presence of the NMe-aromatic amino acid immonium ion which resides in position 2. NMePhe, NMeTyr, and NMe-Cl-Tyr immonium ions can be seen at $m / z$ 134.0962, $\mathrm{m} / z$ 150.0910, and $m / z$ 184.0518, respectively.

The four investigated M. aeruginosa strains (CPCC 299, CPCC 300, CPCC 632, CPCC 633) produce diverse and complex mixtures of cyanopeptolins (Table 3.6). M. aeruginosa CPCC 300 varies from M. aeruginosa CPCC 299, CPCC 632, and CPCC 633 as it produces cyanopeptolins with the partial amino acid sequence, Lxx-Ahp whereas the other three strains produce cyanopeptolins with the partial amino acid sequence, Phe-Ahp (Figure 3.5). These congeners have been differentiated based on product ions patterns and result in large enough structural variation that they cluster separately within the molecular network. 

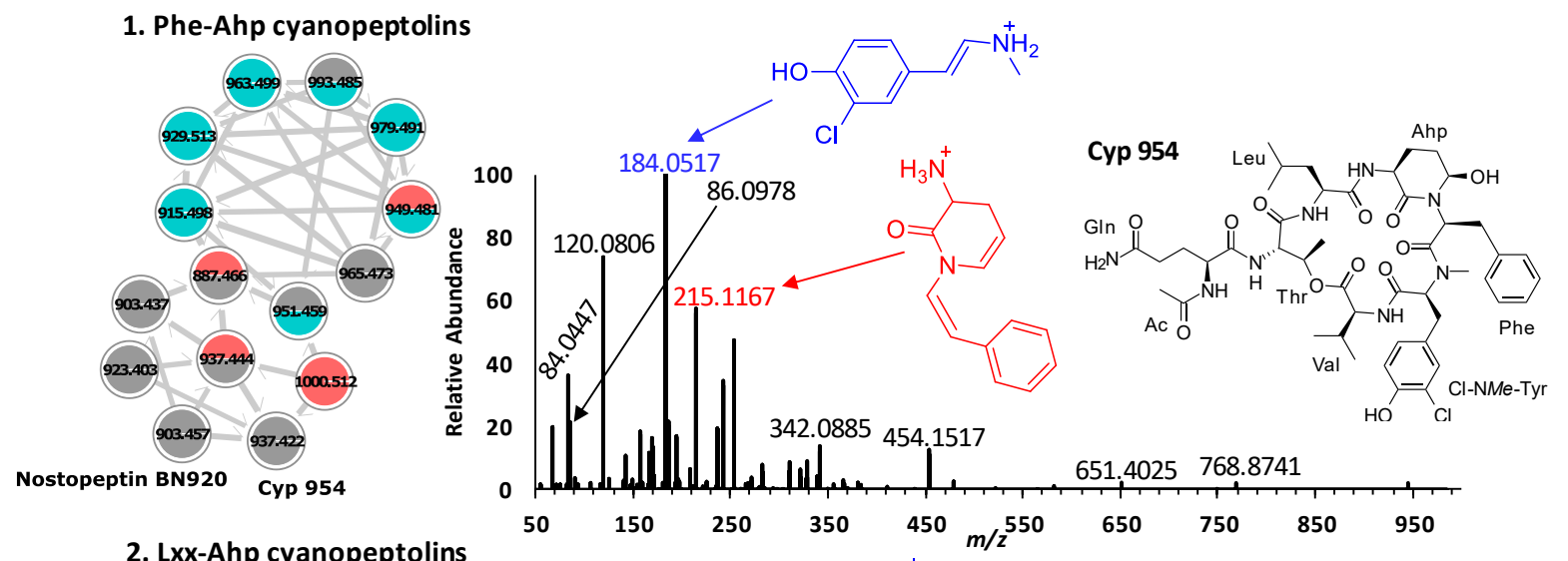

2. Lxx-Ahp cyanopeptolins
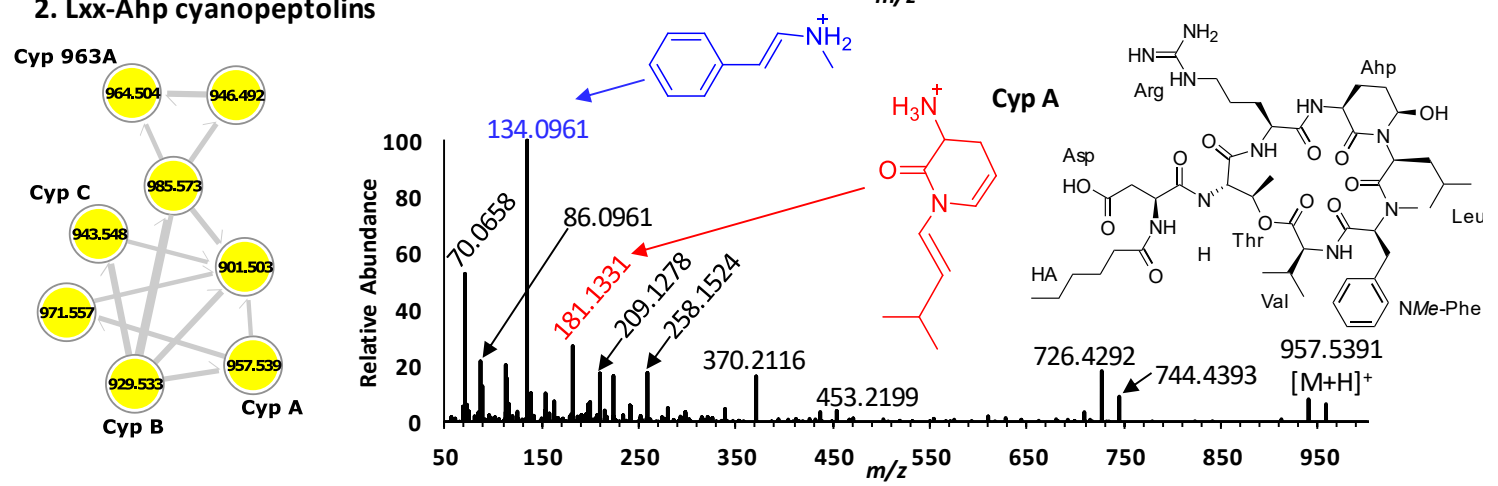

Figure 3.5: The two cyanopeptolin GNPS molecular network clusters, Phe-Ahp cyanopepetolins, cluster \#1 (top left) and Lxx-Ahp cyanopeptolins, cluster \#2 (bottom left) and representative MS/MS spectra for congeners within these subclasses. The chemical structures of cyanopeptolin 954 (top right) and cyanopeptolin A (bottom right) are examples of each subclass. Key product ions to differentiate the subclasses are highlighted in the MS/MS spectra. For Phe-Ahp cyanopeptolins, $m / z 184.0517$ (NMe-Cl-Tyr), $215.1167\left(\left[\mathrm{Ahp}-\mathrm{Phe}-\mathrm{CO}-\mathrm{H}_{2} \mathrm{O}\right]^{+}\right)$are present and for Lxx-Ahp cyanopeptolins $m / z 134.0961$ (NMePhe), 181.1331 ([Ahp-Lxx-CO- $\left.\mathrm{H}_{2} \mathrm{O}\right]^{+}$).

M. aeruginosa CPCC 300 produced four previously reported cyanopeptolins: A, B, C, and 963A

(Martin et al., Bister et al.). These metabolites share the partial amino acid sequence, NMePheLeu-Ahp with variation arising by amino acid substitution in any of the surrounding positions. Within the network cluster, there are four additional variants that do not have literature or database matches to metabolites with the partial amino acid sequence Lxx-Ahp, thus correspond to new potential cyanopeptolin variants (Table 3.1). 
M. aeruginosa CPCC 632 produced two previously reported cyanopeptolins, nostopeptin BN920 and its chlorinated form cyanopeptolin 954 (Von Elert et al., 2005). This strain also produces the widest range of variants with other possible congener partial amino acid sequences: NMePhe-PheAhp, NMeTyr-Phe-Ahp, and NMe-Cl-Tyr-Phe-Ahp. M. aeruginosa CPCC 299 exclusively produces cyanopeptolins with the partial amino acid sequence NMePhe-Phe-Ahp whereas CPCC 633 produces mixtures of cyanopeptolins with either $\mathrm{N} M e \mathrm{Tyr}$ or $\mathrm{N} M e$ Phe within position 2. Halogenation of the NMeTyr and substitution to $\mathrm{NMePhe}$ does not merit the generation of a third cyanopeptolin cluster, thus these variants are all grouped together and labeled for the partial sequence they share, Phe-Ahp (Table 3.1).

Table 3.1: Summary of all cyanopeptolin variants within the GNPS molecular network.

\begin{tabular}{|c|c|c|c|c|c|c|}
\hline $\begin{array}{c}\text { Cluster } \\
\#\end{array}$ & Name & $\begin{array}{c}m / z \\
{\left[\mathbf{M}+\mathbf{H}-\mathbf{H}_{2} \mathbf{O}\right]^{+}}\end{array}$ & Formula & $\begin{array}{l}\text { Error } \\
(\mathrm{ppm})\end{array}$ & $\overline{\text { AA1 }}$ & $\mathbf{A A 2}$ \\
\hline 1 & Cyanopeptolin 904 & 887.4652 & $\mathrm{C}_{46} \mathrm{H}_{64} \mathrm{~N}_{8} \mathrm{O}_{11}$ & -1.08 & $\mathrm{~N} M e$ Phe & Phe \\
\hline 1 & Cyanopeptolin 920 & 903.4367 & $\mathrm{C}_{43} \mathrm{H}_{65} \mathrm{~N}_{8} \mathrm{O}_{12} \mathrm{Cl}$ & -1.37 & $\mathrm{NMe-Cl-Tyr}$ & Phe \\
\hline 1 & Nosptopeptin BN920 & 903.4587 & $\mathrm{C}_{46} \mathrm{H}_{64} \mathrm{~N}_{8} \mathrm{O}_{12}$ & 0.10 & $\mathrm{~N} M e \mathrm{Tyr}$ & Phe \\
\hline 1 & Cyanopeptolin 932 & 915.4972 & $\mathrm{C}_{48} \mathrm{H}_{68} \mathrm{~N}_{8} \mathrm{O}_{11}$ & -0.29 & $\mathrm{~N} M e \mathrm{Tyr}$ & Phe \\
\hline 1 & Cyanopeptolin 940 & 923.4039 & $\mathrm{C}_{45} \mathrm{H}_{65} \mathrm{~N}_{8} \mathrm{O}_{12} \mathrm{Cl}$ & -0.45 & $\mathrm{NMe}-\mathrm{Cl}-\mathrm{Tyr}$ & Phe \\
\hline 1 & Cyanopeptolin 946 & 929.5116 & $\mathrm{C}_{49} \mathrm{H}_{70} \mathrm{~N}_{8} \mathrm{O}_{11}$ & -1.63 & $\mathrm{~N} M e \mathrm{Tyr}$ & Phe \\
\hline 1 & Cyanopeptolin 954 & 937.4217 & $\mathrm{C}_{46} \mathrm{H}_{63} \mathrm{~N}_{8} \mathrm{O}_{12} \mathrm{Cl}$ & -0.43 & $\mathrm{NMe}-\mathrm{Cl}-\mathrm{Tyr}$ & Phe \\
\hline 1 & Cyanopeptolin 954B & 937.4441 & $\mathrm{C}_{49} \mathrm{H}_{62} \mathrm{~N}_{8} \mathrm{O}_{12}$ & -1.41 & NMePhe & Phe \\
\hline 1 & Cyanopeptolin 966 & 949.4821 & $\mathrm{C}_{51} \mathrm{H}_{66} \mathrm{~N}_{8} \mathrm{O}_{11}$ & 0.29 & NMePhe & Phe \\
\hline 1 & Cyanopeptolin 968 & 951.4596 & $\mathrm{C}_{50} \mathrm{H}_{64} \mathrm{~N}_{8} \mathrm{O}_{12}$ & -1.55 & NMeTyr & Phe \\
\hline 1 & Cyanopeptolin 980 & 963.4964 & $\mathrm{C}_{52} \mathrm{H}_{68} \mathrm{~N}_{8} \mathrm{O}_{11}$ & -1.04 & $\mathrm{~N} M e \mathrm{Tyr}$ & Phe \\
\hline 1 & Cyanopeptolin 982 & 965.4525 & $\mathrm{C}_{48} \mathrm{H}_{67} \mathrm{~N}_{8} \mathrm{O}_{12} \mathrm{Cl}$ & 1.19 & $\mathrm{NMe}-\mathrm{Cl}-\mathrm{Tyr}$ & Phe \\
\hline 1 & Cyanopeptolin 992 & 979.4930 & $\mathrm{C}_{52} \mathrm{H}_{68} \mathrm{~N}_{8} \mathrm{O}_{12}$ & 0.73 & NMeTyr & Phe \\
\hline 1 & Cyanopeptolin 1010 & 993.4856 & $\mathrm{C}_{50} \mathrm{H}_{71} \mathrm{~N}_{8} \mathrm{O}_{12} \mathrm{Cl}$ & 0.08 & $\mathrm{NMe-Cl-Tyr}$ & Phe \\
\hline 1 & Cyanopeptolin 999 & 1000.5120 & $\mathrm{C}_{51} \mathrm{H}_{69} \mathrm{~N}_{9} \mathrm{O}_{12}$ & -1.34 & $\mathrm{~N} M e$ Phe & Phe \\
\hline 2 & Cyanopeptolin 900 & $901.5031 *$ & $\mathrm{C}_{44} \mathrm{H}_{68} \mathrm{~N}_{8} \mathrm{O}_{12}$ & 0.17 & $\mathrm{NMePhe}$ & Lxx \\
\hline 2 & Cyanopeptolin B & 929.5327* & $\mathrm{C}_{46} \mathrm{H}_{72} \mathrm{~N}_{8} \mathrm{O}_{12}$ & -1.66 & $\mathrm{NMePhe}$ & Leu \\
\hline 2 & Cyanopeptolin $\mathrm{C}$ & 943.5481* & $\mathrm{C}_{47} \mathrm{H}_{74} \mathrm{~N}_{8} \mathrm{O}_{12}$ & -1.90 & $\mathrm{~N} M e \mathrm{Phe}$ & Leu \\
\hline 2 & Cyanopeptolin 945 & 946.4917* & $\mathrm{C}_{49} \mathrm{H}_{67} \mathrm{~N}_{7} \mathrm{O}_{12}$ & -0.36 & $\mathrm{NMePhe}$ & Lxx \\
\hline 2 & Cyanopeptolin A & 957.5391* & $\mathrm{C}_{46} \mathrm{H}_{72} \mathrm{~N}_{10} \mathrm{O}_{12}$ & -1.35 & NMePhe & Leu \\
\hline 2 & Cyanopeptolin 963A & $964.5040^{*}$ & $\mathrm{C}_{49} \mathrm{H}_{69} \mathrm{~N}_{7} \mathrm{O}_{13}$ & 1.53 & $\mathrm{NMePhe}$ & Leu \\
\hline 2 & Cyanopeptolin 970 & $971.5565^{*}$ & $\mathrm{C}_{47} \mathrm{H}_{74} \mathrm{~N}_{10} \mathrm{O}_{12}$ & 0.46 & $\mathrm{NMePhe}$ & Lxx \\
\hline 2 & Cyanopeptolin 984 & $985.5725^{*}$ & $\mathrm{C}_{48} \mathrm{H}_{76} \mathrm{~N}_{10} \mathrm{O}_{12}$ & 0.61 & $\mathrm{~N} M e$ Phe & Lxx \\
\hline
\end{tabular}

${ }^{*}[\mathrm{M}+\mathrm{H}]^{+}$

$\underline{3.1 .3 \mathrm{c} \text { Molecular network analysis: Anabaenopeptins }}$ 
Anabaenopeptins (APs) are a group of hexapeptide cyanopeptides, with a cyclic core composed of five residues and one amino acid in an exo position. This group of cyanopeptides is of interest as its congeners demonstrate a similar mechanism of toxicity as MCs, potent inhibition of protein phosphatases (Janssen et al., 2019). APs share a ureido linkage for cyclization by a lysine residue at position 2 . This linkage is common between all congeners within the group and connects the exo residue at position 1 to the residue at position 3 . All other positions, aside from the lysine, are variable making this group very structurally diverse (Spoof et al., 2016). The dominant common product ions observed within the MS/MS of anabaenopeptins are $\mathrm{m} / \mathrm{z} 84.0812$ and $\mathrm{m} / \mathrm{z} 129.1018$, characteristic of the lysine residue. All congeners detected here contain an NMe-Ala at position 5 with the diagnostic product ion $m / z 58.0658$ in all AP MS/MS spectra. Two of the five Microcystis strains studied produced similar mixtures of APs, M. aeruginosa CPCC 632 and CPCC 633 (Table S1, Figure 3.6). 


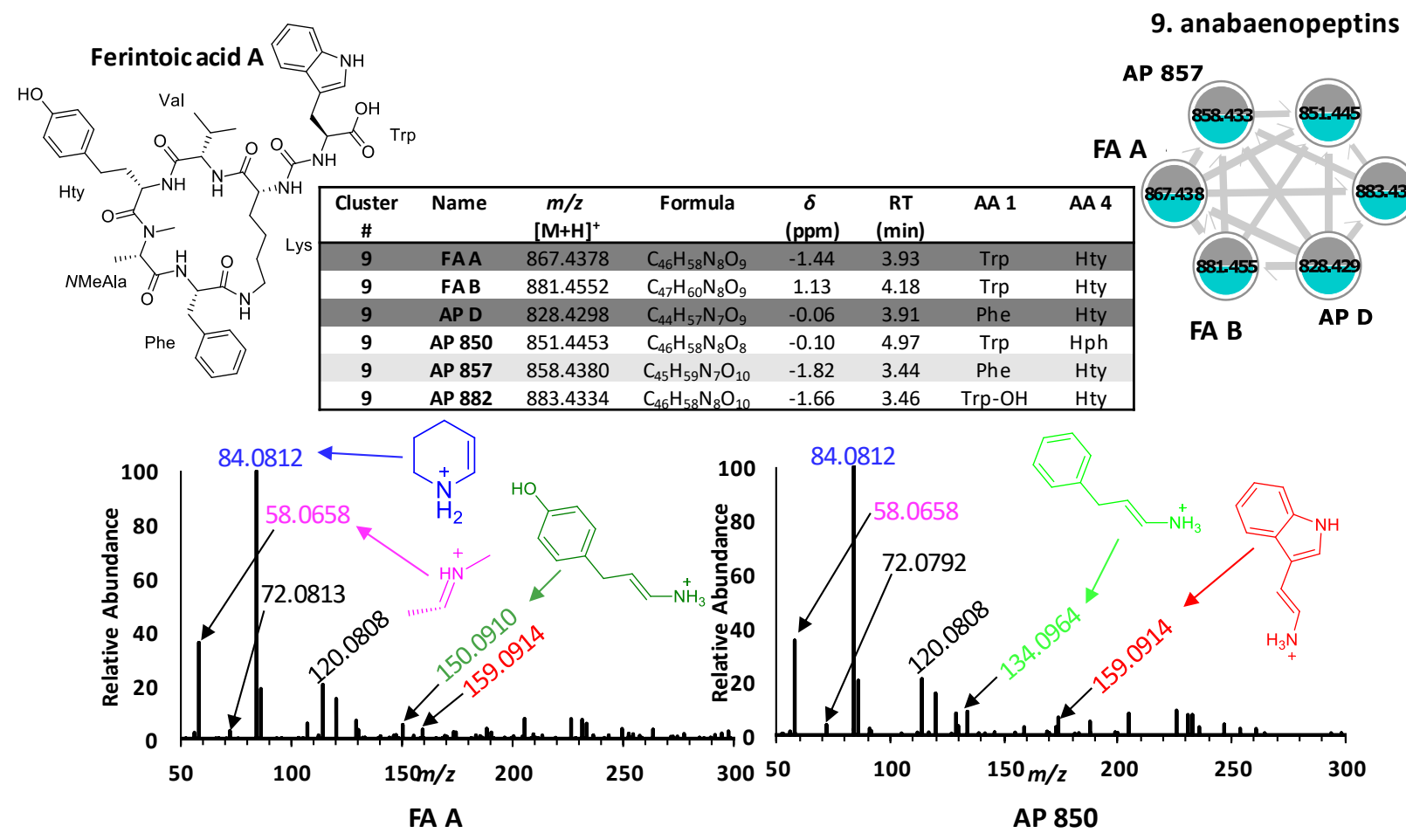

Figure 3.6: Representative chemical structure of the AP congener, Ferintoic acid A (top left). The AP GNPS molecular network cluster \# 9 (top right) showing congeners produced by M. aeruginosa CPCC 632 (blue) and CPCC 633 (grey) with summary table of MS information for the detected AP congeners. The MS/MS of Ferintoic acid A (bottom left) and AP 850 (bottom right) with diagnostic product ions and their putative structures, $m / z 58.0658$ (NMe-Ala), $m / z 72.0792$ (Val), $m / z$ 84.0812 (Lys), m/z 120.0835 (Phe), m/z 150.0910 (Homo-Tyr; Hty), m/z 134.0964 (HomoPhe; Hph), and $m / z 159.0914$ (Trp).

Ferintoic acid A and B (FA A and FA B), AP D, and AP NZ857 are detected congeners that have been previously reported (Spoof et al., 2015). The identity of these previously reported congeners was confirmed based on diagnostic product ions within the MS/MS. For example, immonium ions that confirmed the identity of AP D are $m / z 58.0658$ (NMeAla), $m / z 72.0813$ (Val), $m / z$ 84.0812 (Lys), $m / z 120.0808$ (Phe), and $m / z 150.0909$ (Hty). Two AP congeners within the network cluster \#9 could not be matched to a previously identified metabolites through library or database searches and are considered potential new APs (Figure 3.6). MS analysis shows AP 850 is 15.9925 mass units less than FA A suggesting the loss of an oxygen atom, which is accounted for within the 
MS/MS as a substitution of $m / z 150.0906$ of Hty in FA A to $m / z 134.0962$ of Hph in AP 850 (Figure 3.6). MS analysis shows AP 882 is 15.9956 mass units larger than FA A suggesting the gain of an oxygen atom. This is explained within the MS/MS as a substitution of $m / z 159.0914$ of Trp in the exo position of FA A to $m / z 175.0863$ of 5-hydroxy-Trp of AP 882. These substitutions of one aromatic amino acid to another is common and suggests the synthetic enzyme has low specificity that aids in increased chemical diversity for APs.

\section{$\underline{\text { 3.1.3d Molecular network analysis: Microginins }}$}

Microginins (MGs) are a group of linear cyanopeptides containing 4-6 amino acid residues and a characteristic N-terminal 3-amino-2-hydroxydecanoic acid (Ahda) from a polyketide synthesis pathway. Variation in this characteristic residue occurs with a shorter chain length to 3-amino-2hydroxyoctanoic acid (Ahoa) as well as different chlorination states of the terminal end of the polyketide moiety. Characteristic product ions resulting from the fragmentation of these characteristic microginin N-terminal polyketide moieties include $\mathrm{m} / z$ 128.1433, indicative of the Ahda functionality. A mass increase of 33.9606 units to $\mathrm{m} / \mathrm{z} 162.1039$ and again to $\mathrm{m} / \mathrm{z} 196.0645$ are characteristic of mono- and di-chlorinated Ahda, respectively. The shorter chain congeners generate similar product ions at $\mathrm{m} / \mathrm{z} 100.1122, \mathrm{~m} / \mathrm{z} 134.0727, \mathrm{~m} / \mathrm{z} 168.0338$, indicative of the non, mono-, and di-chlorinated Ahoa residues, respectively (Strangman \& Wright, 2016). 


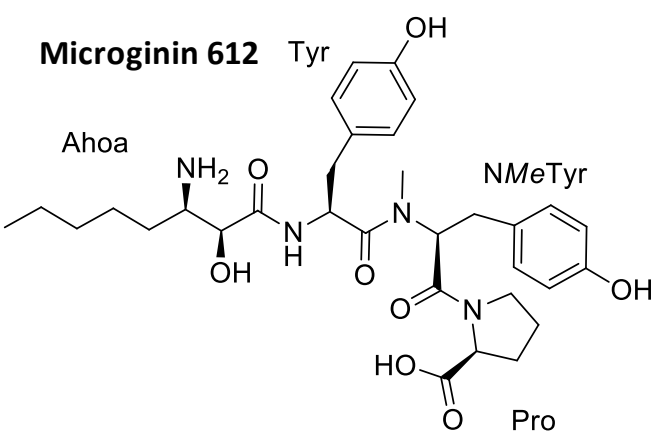

\begin{tabular}{|c|c|c|c|c|c|c|}
\hline $\begin{array}{c}\text { Cluster } \\
\# \\
\end{array}$ & Name & $\begin{array}{c}m / z \\
{[\mathrm{M}+\mathrm{H}]^{+}}\end{array}$ & Formula & $\begin{array}{c}\delta \\
(\mathrm{ppm})\end{array}$ & $\begin{array}{c}\text { RT } \\
\text { (min) }\end{array}$ & AA 1 \\
\hline 7 & Mg 612 & 613.3225 & $\mathrm{C}_{32} \mathrm{H}_{44} \mathrm{~N}_{4} \mathrm{O}_{8}$ & -1.11 & 2.52 & Ahoa \\
\hline 7 & Mg 646 & 647.2826 & $\mathrm{C}_{32} \mathrm{H}_{43} \mathrm{~N}_{4} \mathrm{O}_{8} \mathrm{Cl}$ & -2.22 & 2.56 & Cl-Ahoa \\
\hline 7 & Mg 680 & 681.2441 & $\mathrm{C}_{32} \mathrm{H}_{42} \mathrm{~N}_{4} \mathrm{O}_{8} \mathrm{Cl}_{2}$ & -1.44 & 2.65 & $\mathrm{Cl}_{2}$-Ahoa \\
\hline 7 & Mg 626 & 627.3374 & $\mathrm{C}_{33} \mathrm{H}_{46} \mathrm{~N}_{4} \mathrm{O}_{8}$ & 0.49 & 2.70 & NMe-Ahoa \\
\hline 7 & Mg 628 & 629.3184 & $\mathrm{C}_{32} \mathrm{H}_{44} \mathrm{~N}_{4} \mathrm{O}_{9}$ & 0.11 & 2.31 & Cl-Ahoa \\
\hline 7 & Mg 630 & 631.2891 & $\mathrm{C}_{32} \mathrm{H}_{43} \mathrm{~N}_{4} \mathrm{O}_{7} \mathrm{Cl}$ & -0.32 & 2.60 & $\mathrm{Cl}-\mathrm{Ao}$ \\
\hline 7 & Mg 640 & 641.3535 & $\mathrm{C}_{34} \mathrm{H}_{48} \mathrm{~N}_{4} \mathrm{O}_{8}$ & -1.54 & 2.75 & Ahda \\
\hline 7 & Mg 660 & 661.2985 & $\mathrm{C}_{33} \mathrm{H}_{45} \mathrm{~N}_{4} \mathrm{O}_{8} \mathrm{Cl}$ & -1.66 & 2.70 & $\mathrm{NMe}-\mathrm{Cl}$-Ahoa \\
\hline 7 & Mg 664 & 665.2503 & $\mathrm{C}_{32} \mathrm{H}_{42} \mathrm{~N}_{4} \mathrm{O}_{7} \mathrm{Cl}_{2}$ & 0.41 & 3.04 & $\mathrm{Cl}_{2}-\mathrm{Ao}$ \\
\hline 7 & Mg 674 & 675.3148 & $\mathrm{C}_{34} \mathrm{H}_{47} \mathrm{~N}_{4} \mathrm{O}_{8} \mathrm{Cl}$ & -1.06 & 2.72 & $\mathrm{Cl}$-Ahda \\
\hline 7 & Mg 694 & 695.2599 & $\mathrm{C}_{33} \mathrm{H}_{44} \mathrm{~N}_{4} \mathrm{O}_{8} \mathrm{Cl}_{2}$ & -0.39 & 2.89 & $\mathrm{NMe}-\mathrm{Cl}_{2}-\mathrm{Ahoa}$ \\
\hline 7 & Mg 714 & 715.2053 & $\mathrm{C}_{32} \mathrm{H}_{41} \mathrm{~N}_{4} \mathrm{O}_{8} \mathrm{Cl}_{3}$ & -1.36 & 2.86 & $\mathrm{Cl}_{3}$-Ahoa \\
\hline
\end{tabular}
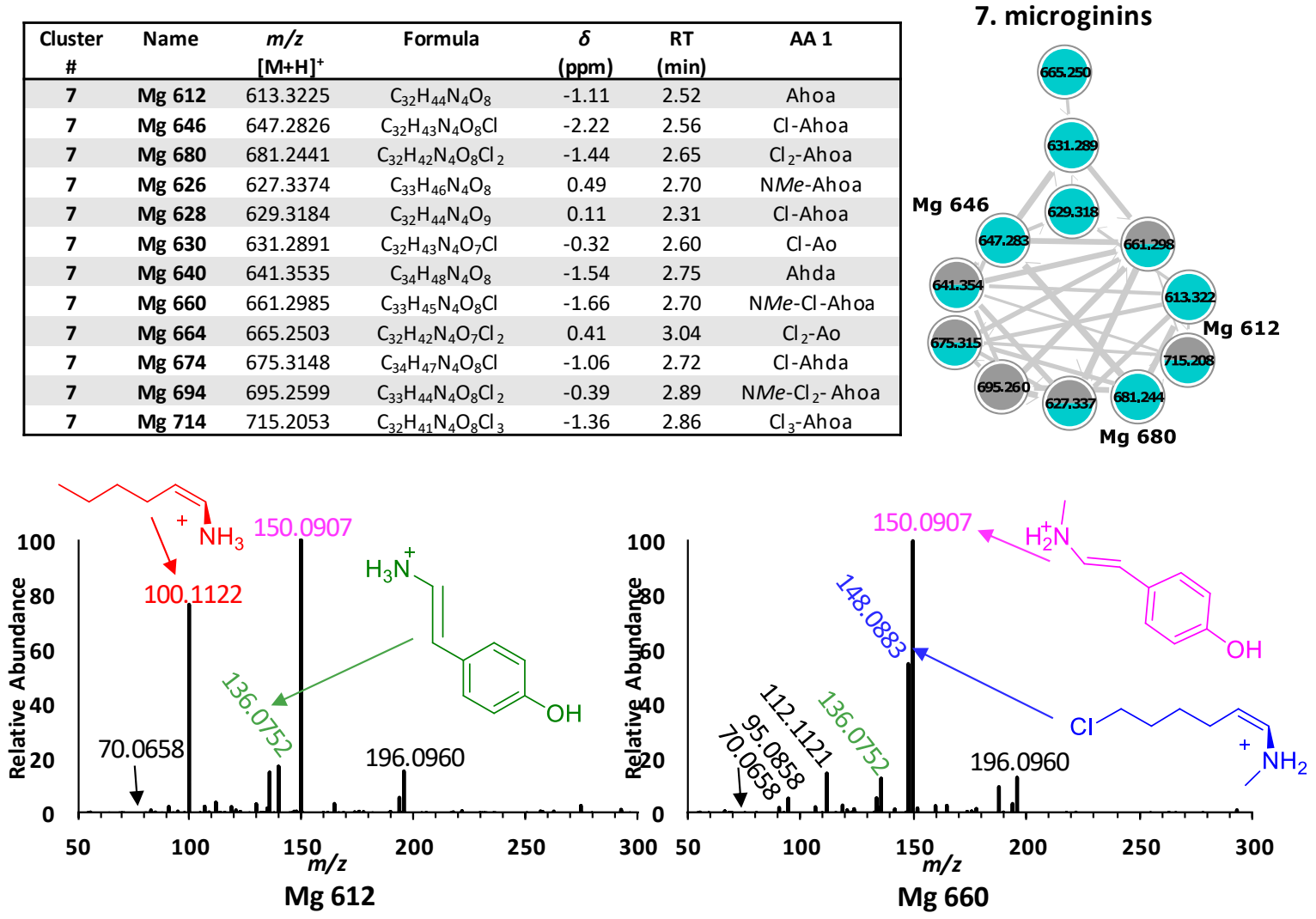

Figure 3.7: Representative chemical structure of MG congener, microginin 612 (top left). LC-MS spectrum (top left) of co-eluting MG congeners (MG 612; $m / z 613.3225[\mathrm{M}+\mathrm{H}]^{+}, \mathrm{MG} 646 ; \mathrm{m} / z$ $646.2826[\mathrm{M}+\mathrm{H}]^{+}$, and $\mathrm{MG} 680 ; m / z 681.2441[\mathrm{M}+\mathrm{H}]^{+}$) with varying chlorination states seen as a mass difference of 1 chlorine atom (33.98). The MG GNPS molecular network cluster \#7 showing congeners produced by M. aeruginosa CPCC 632 (grey) and CPCC 633 (blue). The MS/MS of MG 612 (bottom left) and MG 660 (bottom right) with diagnostic product ions labelled, $\mathrm{m} / z$ 100.1122 (Ahoa), $m / z$ 148.0833 (Cl-NMe-Ahoa), as well as product ions $m / z 70.0658$ (Pro), $m / z$ 136.0752 (Tyr) and $m / z 150.0910$ (NMeTyr) used to confirm the structure.

MGs were produced by two M. aeruginosa strains, CPCC 632 and CPCC 633 (Table S1, Figure 3.7). Unlike the APs, the MG mixtures produced by these strains were not the same. The MG 
GNPS network cluster (\#7) is comprised of twelve MG congeners, eleven of which are produced by M. aeruginosa CPCC 633 and six by M. aeruginosa CPCC 632. This highlights similar cyanopeptide profiles for M. aeruginosa CPCC 633 and CPCC 633, that overlapped in the PCA plot (Figure 3.1). Of the twelve MGs identified, three have previously been identified, MG 612, MG 646, and MG 680 and are exclusively produced by M. aeruginosa CPCC 633 (Strangman \& Wright, 2016). These congeners possess the uncommon Ahoa residue and vary only by the chlorination state of the N-terminal polyketide chain. An N-methylated series of molecules akin to these previously reported molecules was produced by both strains, MG 626, MG 660, and MG 694 by an increase in mass units of 14.0159 in each case. Upon investigation of the MS/MS of these series of molecules, it is apparent that those produced by M. aeruginosa CPCC 632 are structural isomers to the series produced by M. aeruginosa CPCC 633 . When comparing the MS/MS of the previously reported MGs (MG 612, MG 646, MG 680) to the series of unknown MG metabolites produced by M. aeruginosa CPCC 632, the diagnostic product ions associated with the Ahoa and the potential chlorination states are present at $\mathrm{m} / \mathrm{z} 100.1122$ (Ahoa), $\mathrm{m} / \mathrm{z}$ 134.0727 (Cl-Ahoa), and $m / z$ 168.0338 ( $\mathrm{Cl}_{2}$-Ahoa). This suggests the methyl addition is elsewhere. Further investigation reveals that in the MS/MS spectra of MG 626, MG 660, and MG 694 produced by M. aeruginosa CPCC 632 there is no immonium ion for $\operatorname{Tyr}(m / z 136.0762)$ only $\mathrm{N} M e \operatorname{Tyr}(m / z$ 150.0912), suggesting both residues within the molecule are NMeTyr. For example, the amino acid sequence for MG 626 is Ahoa-NMeTyr-NMeTyr-Pro.

When analyzing the MS/MS spectra for the unknown MG metabolite series produced by $M$. aeruginosa CPCC 633, the diagnostic product ions associated with Ahoa and the potential chlorination states are not present. Instead, they are replaced by a series of product ions with a mass difference of 14.0156 units, $m / z 114.1278$ (NMe-Ahoa), $m / z$ 148.0883 (NMe-Cl-Ahoa), $m / z$ 
182.0494 ( $\mathrm{NMe}-\mathrm{Cl}_{2}$-Ahoa). This suggest methylation of the amine of the Ahoa as an increase in chain length would not be possible through the polyketide synthase pathway, as well congeners with modification in this manner have been previously reported (Lodin-Friedman \& Carmeli, 2018). For example, the amino acid sequence for MG 626 is NMe-Ahoa-Tyr-NMeTyr-Pro.

Of the twelve MGs grouped within the molecular network; all are tetra-peptides, nine are chlorinated, ten have the octanoic acid terminal chain, and two have the decanoic acid terminal chain (Figure 3.7). Of the ten congeners with the octanoic acid terminal chain, three have methylated amines (NMe-Ahoa), two do not have a hydroxyl on the carbon adjacent to the amine (Amino-octanoic acid, Ahoa), and the remaining five have Ahoa. The isotope pattern as well as retention time gave indication on the level of chlorination of the cyanopeptides. Both strains produced MG variants with Ahda and Ahoa residues with capability for non-, mono-, di-, and trichlorination. Nine of the twelve MGs identified are chlorinated, with all possible chain modifications having a mono-chlorinated congener suggesting chlorination of the chain occurs in mono-substitution steps. Not all chain types have a non-chlorinated state or di-chlorinated state.

M. aeruginosa CPCC 633 produced two amino-octanoic derivatives of the known MGs, MG 680 $\left(\mathrm{m} / z 681.2441[\mathrm{M}+\mathrm{H}]^{+}\right)$and $\mathrm{MG} 646\left(\mathrm{~m} / z 647.2826[\mathrm{M}+\mathrm{H}]^{+}\right)$with a mass difference of 15.9926 less, MG $660\left(\mathrm{~m} / z 661.2985[\mathrm{M}+\mathrm{H}]^{+}\right)$and MG $630\left(\mathrm{~m} / z 631.2891[\mathrm{M}+\mathrm{H}]^{+}\right)$, respectively. A mass difference of this size, suggests the loss of an oxygen atom from each of the previously reported metabolites. Further relatedness is confirmed as these sets of compounds share the isotope pattern of di- and mono-chlorinated, respectively. MG 646 has 4 hydroxyl groups that could be removed for the loss of 15.9926 mass units from the precursor ion, 1 on each residue: Pro, Tyr, NMeTyr, and on the Ahoa. When comparing the MS/MS of MG 646 and MG 630, the product ions for both Tyr residues as well as the Pro are present. The Cl-Ahoa immonium ion is also present but the 
chain breaks at the carbon with the amine and does not include the hydroxyl, suggesting this is the site without a hydroxyl.

\section{$\underline{\text { 3.1.3e Molecular network analysis: Microviridins }}$}

Microviridins (MVs) are tricyclic ribosomally synthesized and post-translationally modified peptides (RiPPs) typically composed of fourteen amino acids with $\omega$-ester and $\omega$-amide bonds between two peptide chains (Rohrlack et al., 2003). These unusual peptides, unlike the other cyanopeptide groups discussed here, are composed of all proteinaceous amino acids with the most reoccurring amino acids between congeners being Tyr, Trp, Phe, Thr, Lys, Asp, Ser, and Glu. The molecular weights of these metabolites are larger (1400-2000 Da range) compared to the other cyanopeptides groups studied. Structural variability is high due to the increased number of positions for amino acid substitution. This structural variability is seen within the molecular network clusters \#3, \#4, \#5 as there are separate clusters to encapsulate all the MV congeners detected (Figure 3.3). All MV nodes within the molecular network are associated with the doubly charged precursor ions. Classification of these molecules as microviridins was done based on retention time range (2-3 minutes), doubly charged precursor ion filtering, and the immonium ions for Trp ( $m / z$ 159.0911), Pro ( $m / z$ 70.0657), and Lys ( $m / z$ 84.0812) within their MS/MS spectra. A large factor that contributes to the dominant immonium ions present within the microviridin MS/MS are attributed to the exo amino acids. MV structures can have up to 4 amino acids that are not cyclized and thus are the first product ions to be generated (Ziemert et al., 2010). 


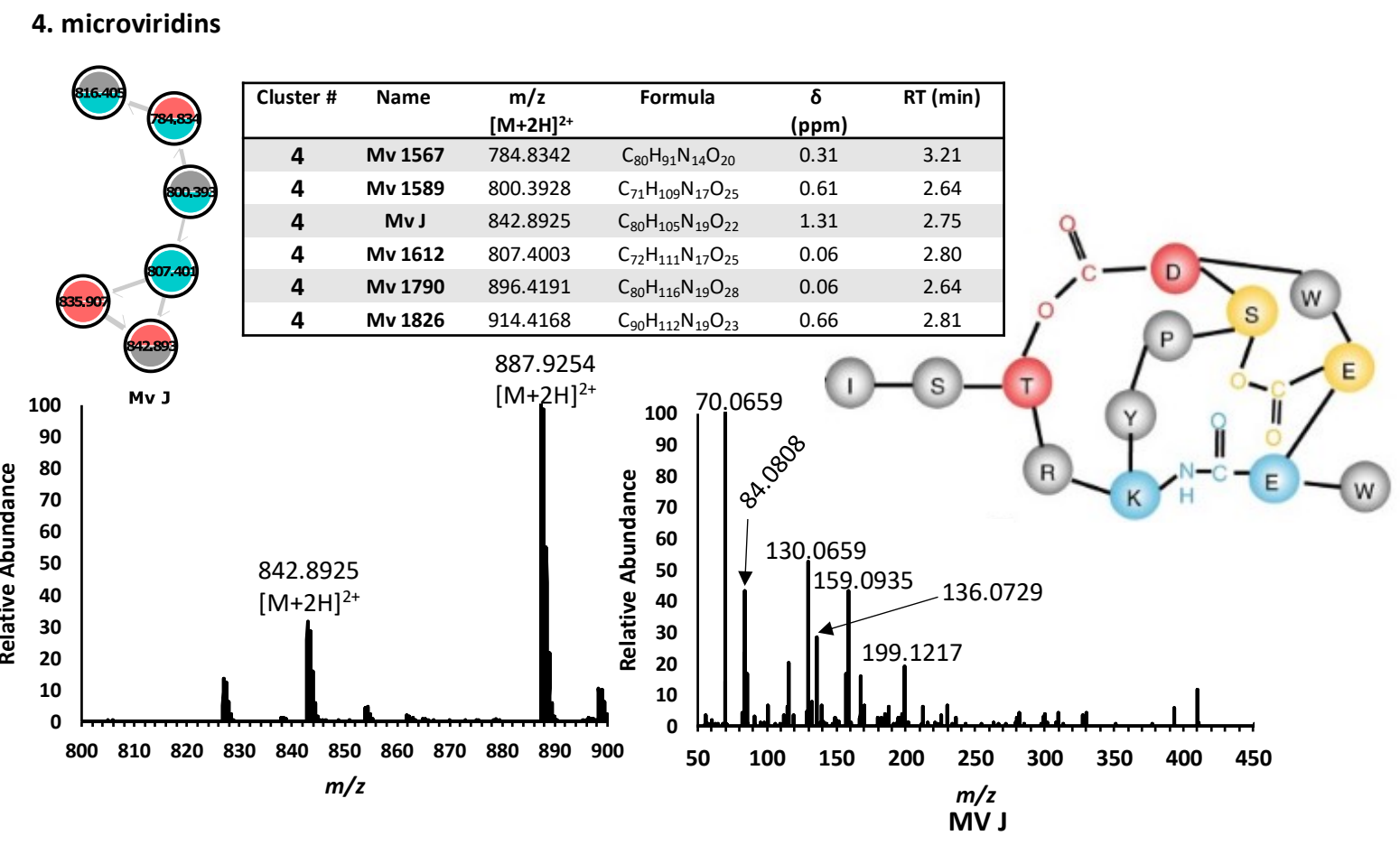

Figure 3.8: The MV GNPS network cluster \#4 (top left) and LC-MS analysis (top right) of congeners produced by $M$. aeruginosa CPCC 299, CPCC 632, and CPCC 633. Full MS scan at 2.76 minutes (bottom left) shows the precursor ion for MV J $\left(m / z 842.8925[\mathrm{M}+2 \mathrm{H}]^{2+}\right)$ which was identified by precursor ion $\mathrm{m} / \mathrm{z}$ and product ions within the MS/MS (bottom right) of $\mathrm{m} / \mathrm{z} 70.0659$ (Pro), $m / z$ 84.0808 (Lys), $m / z 136.0729$ (Tyr), and m/z 159.0935 (Trp). (Zhang, Li, et al., 2018)

Three of the investigated Microcystis strains produced microviridins (MVs), M. aeruginosa CPCC 299, CPCC 632, and CPCC 633. Each strain produces at least one variant within all three (\#3, \#4, \#5) of the MV clusters annotated (Table S1, Figure 3.3). There are eighteen MV congeners present within the molecular network. Two congeners are identified as previously reported, MV H and MV J, and the remainder are potential new MVs (Rohrlack et al., 2003; Ziemert et al., 2010). The identity of the exo amino acids is hypothesized to generate sufficient structural diversity to separate into three MV clusters. Two of the congeners were identified as MV H and MV J through a match with literature by molecular formula and amino acid immonium ions in the MS/MS spectra. MV $\mathrm{H}$ and MV J have similar core amino acids but the identity of the exo amino acids vary. MV $\mathrm{H}$ 
contains Pro, Asp, and two Tyr residues whereas MV J has Ser, Ile, Gln, and Trp residues. Immonium ions of these exo amino acids are present within the MS/MS of these metabolites. Thus, cluster \#4 contains potential MVs with similar exo amino acids as MV H and cluster \#5 contains potential MVs with similar exo amino acids as MV J (Figure 3.8). MV cluster \#3 did not have any library or database matches but contains MV-like metabolites based on the presence of doubly charged precursor ion $m / z$, retention time range, and presence of immonium ions. The MS/MS of metabolites within this cluster share many immonium ions with MV H and MV J, but a few outliers exist such as the immonium ion for Phe at $\mathrm{m} / \mathrm{z} 120.0807$ and an immonium ion at $\mathrm{m} / \mathrm{z} 115.0866$ (NMe-Glu). The exo amino acids for these congeners must vary from those within the congeners of the other network clusters.

\section{$\underline{\text { 3.1.3f Molecular network analysis: Aeruginosins }}$}

Aeruginosins (ASs) are small linear tetrapeptides that contain the characteristic 2-carboxy-6hydroxyoctahydroindole (Choi), a C-terminal Arg or Arg-derivative, and an N-terminal hydroxyphenyl lactic acid (Hpla) with varying substitution of the aromatic ring such as halogenation in the meta positions. Variation increases with O-R modifications of the Choi moiety such as a sulfate group, the cyclization of the Arg to an argal, and finally amino acid substitutions within position 2. Within the MS/MS of AS metabolites, diagnostic product ions are generated from the characteristic Choi moiety, the product ion of Choi at $\mathrm{m} / \mathrm{z} 140.1064$ and the dehydrated form 18.0102 mass units less at $m / z 122.0962$ (Welker et al., 2006).

Two M. aeruginosa strains, CPCC 300 and CPCC 632, produced different mixtures of ASs (Table S1, Figure 3.9). None of the AS variants within the molecular network matched library or database searches suggesting five new potential ASs. The clusters for M. aeruginosa CPCC 300 and CPCC 632 did not group together indicating structural variability between the congeners produced by 
these strains. All congeners were confirmed to be within the AS group based on the presence of product ions characteristic of the Choi moiety.

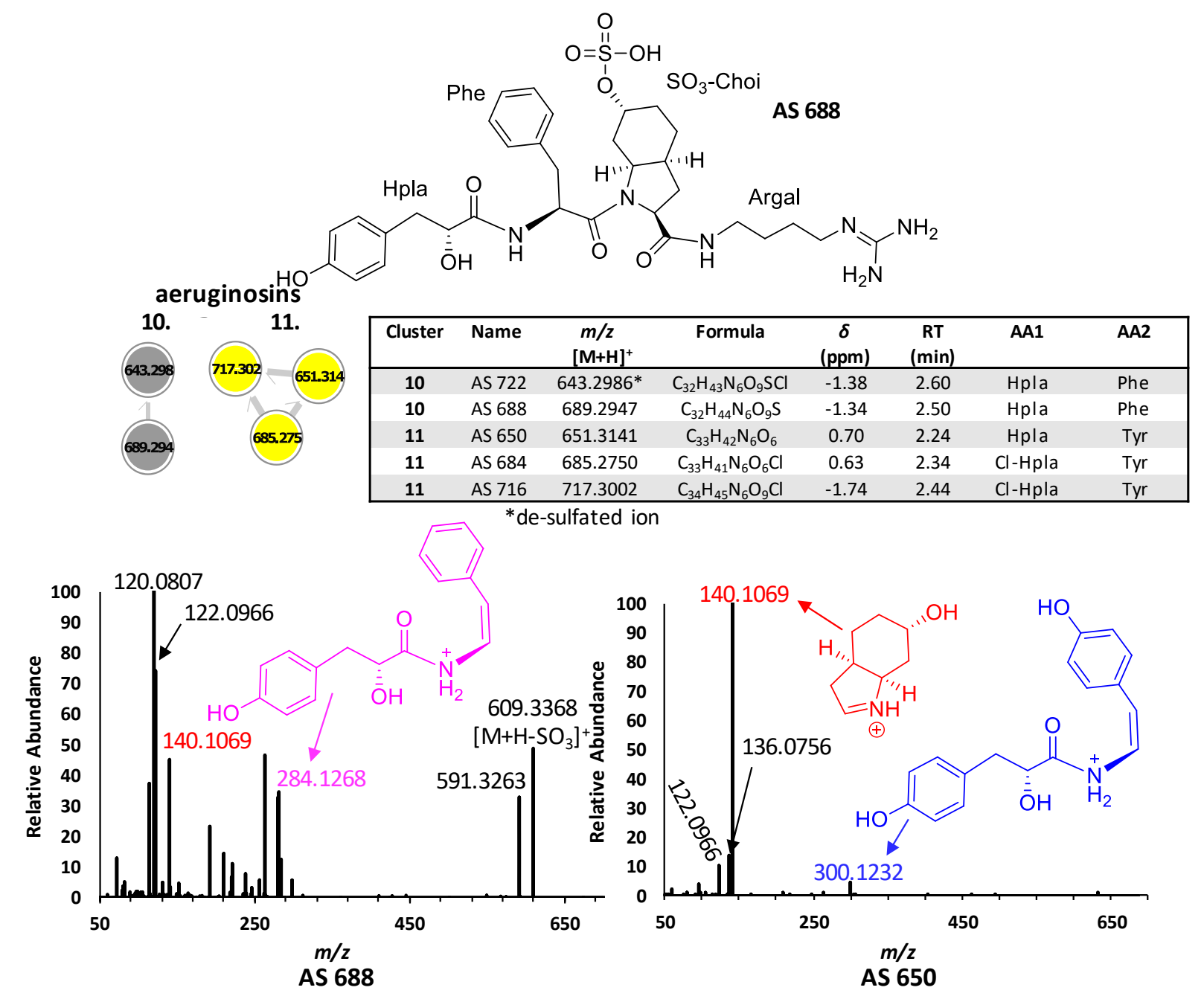

Figure 3.9: The AS GNPS molecular network clusters, \#10 for M. aeruginosa CPCC 632 (grey) and \#11 for M. aeruginosa CPCC 300 (yellow) from the total GNPS network and the congener information. The MS/MS spectrum of AS 688 (bottom left) and AS 650 (bottom right) with diagnostic product ions $\mathrm{m} / \mathrm{z} 140.1069$ and $\mathrm{m} / \mathrm{z} 122.0966$ of the Choi functionality. Further grouping of this chemical group is done with Phe $(\mathrm{m} / \mathrm{z} 120.0807)$ or Tyr $(\mathrm{m} / \mathrm{z} 136.0756)$ substitutions in position 2. Sulfonation of the Choi is possible and visible in the MS as well as the MS/MS as a neutral loss of $79.9571\left(\left[\mathrm{M}+\mathrm{H}-\mathrm{SO}_{3}\right]^{+}\right)$. Partial amino acid sequence was concluded with the product ion corresponding to [Hpla-Residue $2-\mathrm{CO}]^{+}$. For chemicals in cluster 10 contain the sequence [Hpla-Phe $-\mathrm{CO}]^{+}$at $\mathrm{m} / z$ 284.1268 and for chemicals in cluster 11 contain the sequence either sequence [Cl-Hpla-Tyr $-\mathrm{CO}]^{+}$at $m / z 334.0838$ or [Hpla-Tyr $\left.-\mathrm{CO}\right]^{+}$at $m / z$ 300.1232. 
M. aeruginosa CPCC 300 produced three AS variants that contain Tyr within position 2 as well as an Argal (cyclic Arg) in position 4. The Tyr residue is confirmed by the presence of the presence of the Tyr immonium ion in all three fragmentation spectra at $\mathrm{m} / \mathrm{z} 136.0756$. The substitution at the C-terminal position was determined as Argal for the ASs produced by M. aeruginosa CPCC 300 as the immonium ion for $\operatorname{Arg}(m / z 114.1025)$ was not present within the three MS/MS spectra. Upon investigation of the isotope pattern, two of the congeners (AS 684 and AS 650) are singly chlorinated and AS 650 is not chlorinated yet has a mass 33.9606 units less than AS 684 suggesting this metabolite is the non-chlorinated version of AS 684. A $\mathrm{b}_{2}$ fragment within the MS/MS of AS 650 confirms the partial amino acid sequence as Hpla-Tyr at $\mathrm{m} / \mathrm{z} 300.1232$ (Welker et al., 2006). Chlorination within AS 684 and AS 716 on the Hpla is confirmed with the akin fragment 33.9606 mass units greater signifying Cl-Hpla-Tyr at $\mathrm{m} / \mathrm{z} 334.0838$.

M. aeruginosa CPCC 632 produces 2 AS variants that contain a Phe within position 2 as well as an Arg in position 4. The Phe residue was confirmed by the presence of the immonium ion for Phe ( $m / z$ 120.0807) within the MS/MS for the 2 metabolites and the Arg was confirmed with the product ion for $\operatorname{Arg}(m / z$ 114.1025). These metabolites share the characteristic neutral mass loss of $79.9571 \mathrm{~m} / \mathrm{z}$ from the precursor ion within the mass spectrum as well as in the MS/MS indicating sulfonation of the Choi moiety. The increase in $\mathrm{M}+2$ intensity as well as the mass difference between these two congeners being $33.9606 \mathrm{~m} / z$ suggest that AS 688 is not chlorinated and AS 722 is singly chlorinated. The partial sequence Hpla-Phe in AS 688 was confirmed with the product ion $m / z 284.1268$ for the [Hpla-Phe- $\mathrm{CO}+\mathrm{H}]^{+}$. Chlorination of the Hpla is AS 722 is seen with a mass increase of 33.9606 of the aforementioned product ion to $\mathrm{m} / \mathrm{z} 318.0876$ of [Cl-Hpla-Phe$\mathrm{CO}+\mathrm{H}]^{+}$.

\subsection{3g Molecular network analysis: Cyanobactins}


Cyanobactins are a group of highly modified cyanopeptides that contain both proteinaceous amino acids and heterocyclic residues. These residues are a result of a bond between the amino acid side chain heteroatom (oxygen or sulfur atom) from Thr, Ser, and Cys and the preceding amide bond. The enzyme that catalyzes the heterocyclization requires ATP and $\mathrm{Mg}^{2+}$ for activity to synthesize of the oxazoline and thiazolines, as well as the methyl versions (Ge et al., 2019). These residues can be further oxidized to oxazoles and thiazoles which occur in majority of the reported cyanobactins, increasing structural diversity of this chemical group (Leikoski et al., 2013). Further diversity occurs with substitution of proteinaceous amino acids surrounding the heterocycles. These metabolites may be cyclized or linear peptides and will be discussed in terms of cyclic cyanobactins (cyclamides) and linear cyanobactins (aeruginosamides). The opportunity for oxidation/reduction of the heterocyclized atoms, amino acid substitutions, and linear/cyclic cyanobactins results in increased structural diversity and thus fewer diagnostic product ions generated. Thus, there are 3 clusters $(\# 12, \# 13, \# 8)$ to describe all detected cyanobactins (Figure $3.3)$.

M. aeruginosa CPCC 299 and CPCC 300, and M. flos-aquae CPCC 461 produced different mixtures of cyclamides (Figure 3.3). M. aeruginosa CPCC 299 and CPCC 300 produce previously reported aerucyclamide (AC) A and AC C, and M. aeruginosa CPCC 300 also produces AC B and AC D and a related congener of AC C, AC 518 as seen in cluster \#13 (Figure 3.10, Portmann et al., 2008). With the use of HRMS and the occurrence of sulfur atoms within cyclamides, determining molecular formula for the precursor ion as well as product ions containing sulfur is simplified because of the distinct mass defect of sulfur atoms. Based on the MS/MS of the cyclamides within this network cluster, they each have an Me-oxazoline $(m / z 84.0448)$ and Lxx ( $m / z$ 86.0969). For those previously reported and structurally characterized (aerucyclamides A-C), 
we can say that it possesses an Ile residue but for those that are potentially new cyanobactins, we cannot discriminate between the two isobaric residue, Ile and Leu thus we annotate this residue as Lxx. AC A and AC 518 have the partial sequence Tzln-Lxx which is confirmed with the product ions $m / z 86.0064$ (Tzln), $m / z 154.0683$ ([Tzln-Lxx-CONH $2+\mathrm{H}]^{+}$) and $m / z 199.0904$ (Lxx-Tzln). $\mathrm{AC} \mathrm{B}$ and $\mathrm{AC} \mathrm{C}$ have the partial sequence Tzl-Ile which is confirmed with the product ion $\mathrm{m} / \mathrm{z}$ 197.0741 akin to the oxidized product ion seen in the spectra for AC A and AC 518. The evidence suggests that AC 518 is the oxidized congener of AC C, in a similar manner as AC B is the oxidized congener of AC A. The reduced congener contains the Tzln moiety and the oxidized congener contains the Tzl moiety. Other product ions that confirm the structure of AC 518 are $\mathrm{m} / \mathrm{z} 167.0838$ (Val-Ozl) and $m / z 235.1438$ (Val-Ozl-Lxx). The common proteinaceous amino acids and $\mathrm{Me}$ oxazoline present in these metabolites clustered them together within the network. Although, the cyclic nature of these peptides, their uncommon heterocycles, and multiple sites for oxidation/reduction generate a roadblock when searching for variants. 


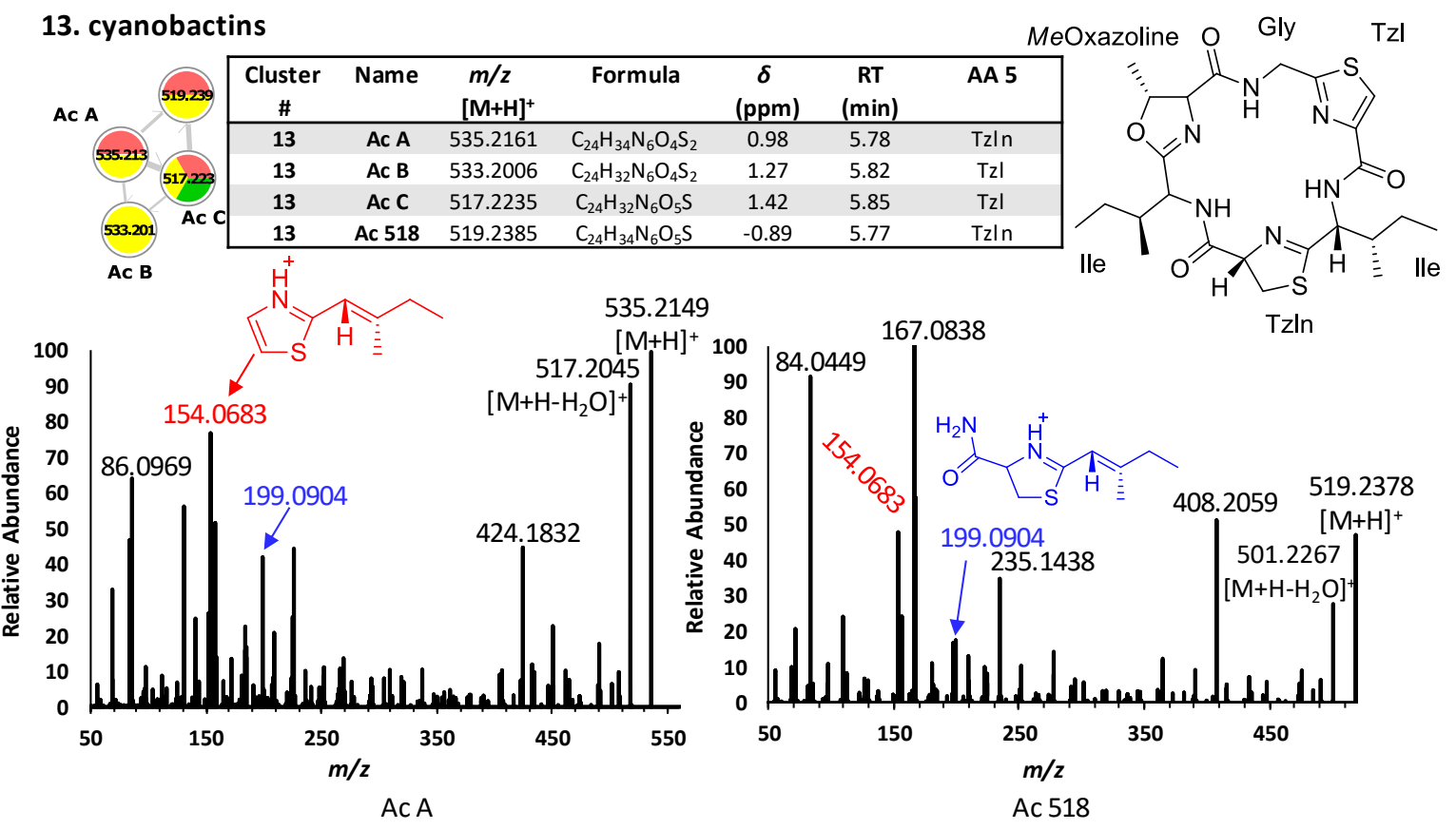

Figure 3.10: Aerucyclamide GNPS network cluster \#13 with LC-MS analysis of the congeners produced by M. aeruginosa CPCC 299 (red), CPCC 300 (yellow), and M. flos-aquae CPCC 461 (green). Representative structure of aerucyclamide A (top right) was confirmed by precursor ion $\mathrm{m} / \mathrm{z}$ and diagnostic product ions from the MS/MS (bottom left), $\mathrm{m} / z$ 86.0969 (Lxx), 154.0683 $\left(\left[\mathrm{Tzln}-\mathrm{Lxx}-\mathrm{CONH}_{2}+\mathrm{H}\right]^{+}\right.$), and 199.0904 (Tzln-Lxx). Potential new aerucyclamide 518 (MS/MS bottom right) was confirmed as reduced aerucyclamide $\mathrm{C}$ with diagnostic ions aligning with Tzln functionality $\mathrm{m} / \mathrm{z} 154.0683$ and 199.0905 , as well as $\mathrm{m} / \mathrm{z} 167.0838$ of the Val-Ozl product ion not present in aerucyclamide A or B.

AC D, which did not appear in the molecular network, has a Met in position 4 and a Phe in position 6 in comparison to the AC A structure (Portmann et al., 2008). This metabolite is product by $M$. aeruginosa CPCC 300 and the identity was confirmed with precursor ion $\mathrm{m} / \mathrm{z}$ and the following product ions $m / z 84.0448$ (MeOxazoline), $m / z 104.0531$ (Met), $m / z 120.0808$ (Phe), and $m / z$ 86.0064 (Tzln). These two substitutions generate enough structural diversity from the other cyclamides that this metabolite did not cluster within the network.

Cluster \# 12 illustrates that M. flos-aquae CPCC 461 produces microcyclamide A and two related reduced congeners Ac 584 and Ac 586 (Figure 3.11, Portmann et al., 2008). This known congener 
has the following hexapeptide sequence: $M e \mathrm{Oxl}-\mathrm{Ile}-\mathrm{Tzl}-\mathrm{MeHis}-\mathrm{Tzl}-\mathrm{Ala}$. This molecule is highly oxidized with many sites for reduction to produce the two potential new congeners reported here. The congeners within this network share the product ion for $\mathrm{MeHis}$ at $m / z 96.0685$ and $\mathrm{MeOxl-}$ Ala at $m / z 153.0656$ confirming the common core structure between these molecules. In the potential new congener, Md 584, the product ion for Ile-Tzl-MeHis-Tzl which is at $m / z 387.1182$ in Md A becomes $m / z 389.1213$ suggesting oxidation of one of the Tzl groups to Tzln. To confirm which Tzl group is oxidized in Md 584, the product ion for the sequence $M e$ His-Tzl with the $m / z$ 235.0645 is present in both MS/MS spectra for Md A and Md 584 suggesting it is the Tzl in position 3 that is oxidized to Tzln (Figure 3.11). In the potential new congener Md 586, both Tzl residues are oxidized to Tzln, thus the mass increase of 4 mass units from the known Md A which is confirmed with the presence of the product ion $\mathrm{m} / \mathrm{z} 391.0996$.

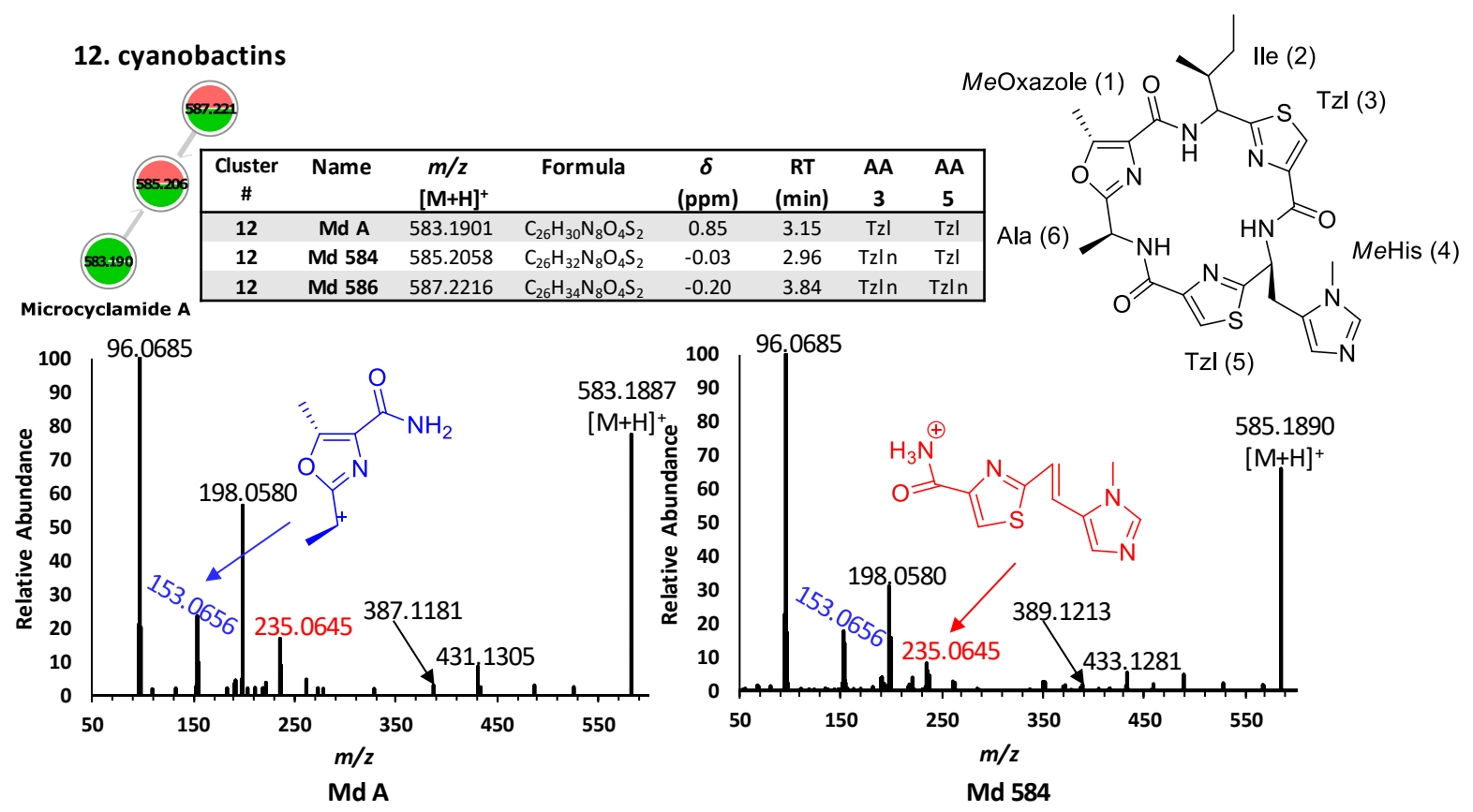

Figure 3.11: Microcyclamide GNPS network cluster \#12 (top left) with LC-MS analysis of the congeners produced by M. aeruginosa CPCC 299 (red) and M. flos-aquae CPCC 461 (green). Representative structure of microcyclamide A $\left(\mathrm{m} / z 583.1887[\mathrm{M}+\mathrm{H}]^{+}\right.$; top right) was confirmed with product ions $\mathrm{m} / \mathrm{z} 261.0798$ (Ala-Tzl-MeHis), 235.0645 ( $M e H i s-T z l), 153.0656$ (MeOxazole- 
Ala), and 96.0685 (MeHis) within the MS/MS (bottom left). Potential new microcyclamides 584 (MS/MS bottom right) contains the reduced Tzl (Tzln) in position 3 and a Tzl in position 5 .

M. aeruginosa CPCC 633 produces linear cyanobactins, known as aeruginosamides (AGs, cluster \#8, Figure 3.12). This subgroup contains a C-terminal prenylated Phe, 1,1-dimethylallyl-Phe (Dma-Phe) and an N-terminal Tzl-OMe (Tzc). These characteristic features provide diagnostic product ions within the MS/MS such as $m / z 188.1428$ for $\left[\right.$ Dma-Phe-CO+H] ${ }^{+}$and $m / z 144.0109$ for Tzc. AG B and AG C are produced by M. aeruginosa CPCC 633 and have been previously reported (Leikoski et al., 2013). These metabolites have two Phe residues and a Pro residue which is confirmed by these immonium ions within their MS/MS at $m / z 120.0807$ (Phe) and $m / z 70.0658$ (Pro). Based on the precursor ion $m / z$, both reported congeners (AG B and AG C) have a related congener with a mass difference of 14.0182 less suggesting loss of a methyl. Demethylation of the Tzc to Tzl-OH (which occurs in Aeruginosamide A, (Leikoski et al., 2013)) is seen in the MS/MS of these two congeners as the absence of the product ion $m / z 144.0109$ and the presence of the product ion $\mathrm{m} / \mathrm{z}$ 129.9955. The loss of the methyl on the Tzc can also be confirmed with the replacement of the product ion $m / z 213.0685$ of $\left[\right.$ Tzc-Pro-CO+H] ${ }^{+}$to 14.0182 mass units less at $m / z$ 199.0503 [Tzl-OH-Pro-CO+H] $]^{+}$. Other congeners within the molecular network cluster \#8 have hydroxyl substitutions seen as the gain of 15.9956 mass units from the precursor ion of a previously reported AG, or as an oxidized Tzc (which occurs in cyclamides, (Portmann, Blom, Gademann, et al., 2008)) seen as the gain in 2 mass units from the precursor ion of a previously reported $\mathrm{AG}$. 


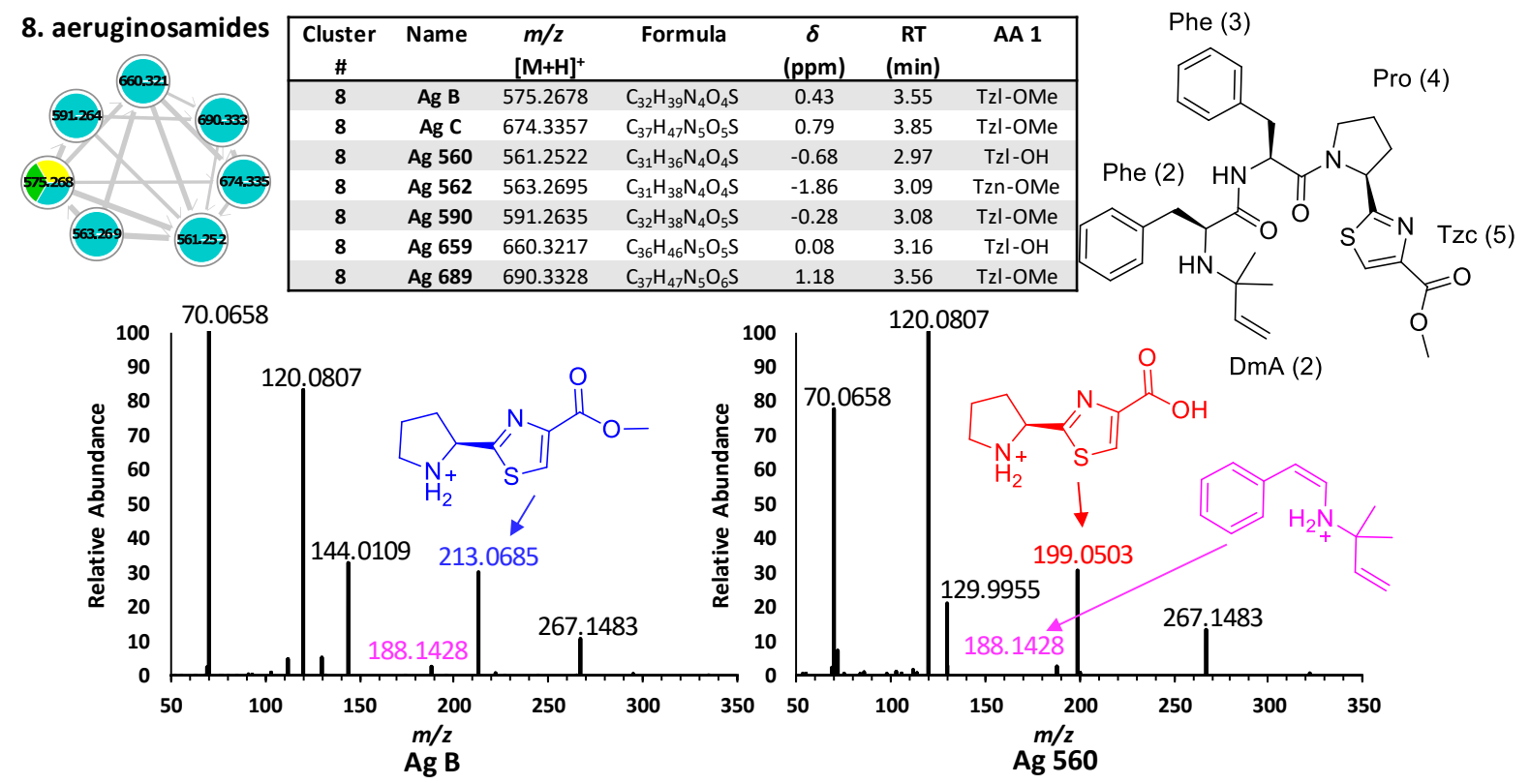

Figure 3.12: The aeruginosamide GNPS molecular network cluster \#8 (top left) with LC-MS analysis of the congeners produced by M. aeruginosa CPCC 632 (blue) and CPCC 300 (yellow), and M. flos-aquae CPCC 461 (green). Representative structure of aeruginosamide B (top right, MS/MS bottom left) was confirmed with the diagnostic product ions $m / z 120.0807$ (Phe), 70.0658 (Pro), 144.0109 (Tzc), and $m / z 213.0685$ (Pro-Tzc). The MS/MS of aeruginosamide 560 (bottom right) shows diagnostic product ions used to elucidate the site of demethylation present at the Tzc to Tzl-OH with the mass difference of 14.0182 at $\mathrm{m} / z 199.0503$.

\subsection{3h Molecular network analysis: unknown cluster \#14}

Interpretation of clusters with no previously reported metabolites or diagnostic product ions were labeled as unknown clusters. Cluster \#14 contains a series of metabolites produced by $M$. aeruginosa CPCC 299 and CPCC 633, that are doubly charged ions within the range $m / z$ 720-770 (molecular ions between 1440-1540 Da, Figure 3.13). The doubly charged ion and the calculated molecular ion matched a few metabolites within the database but comparison of the reported structure and the generated MS/MS, it was concluded that the compounds were not a match. 


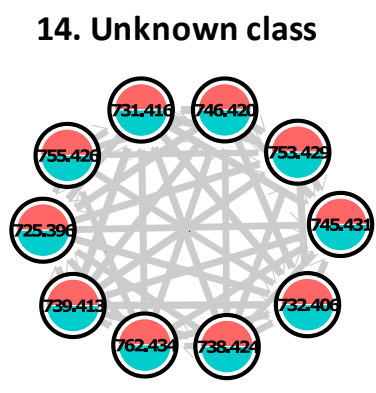

\begin{tabular}{|c|c|c|c|c|c|}
\hline $\begin{array}{c}\text { Cluster } \\
\#\end{array}$ & Name & $\begin{array}{c}m / z \\
{[M+2 H]^{2+}}\end{array}$ & Formula & $\begin{array}{c}\delta \\
(p p m)\end{array}$ & RT (min) \\
\hline 14 & Unk 1448 & 725.3962 & $\mathrm{C}_{73} \mathrm{H}_{106} \mathrm{~N}_{15} \mathrm{O}_{14} \mathrm{~S}$ & 1.02 & 5.38 \\
\hline 14 & Unk 1461 & 731.4159 & $\mathrm{C}_{68} \mathrm{H}_{114} \mathrm{~N}_{15} \mathrm{O}_{18} \mathrm{~S}$ & -0.92 & 5.81 \\
\hline 14 & Unk 1462 & 732.4059 & $\mathrm{C}_{70} \mathrm{H}_{110} \mathrm{~N}_{16} \mathrm{O}_{16} \mathrm{~S}$ & -2.16 & 5.50 \\
\hline 14 & Unk 1474 & 738.4238 & $\mathrm{C}_{69} \mathrm{H}_{116} \mathrm{~N}_{15} \mathrm{O}_{18} \mathrm{~S}$ & -0.75 & 5.81 \\
\hline 14 & Unk 1476 & 739.4133 & $\mathrm{C}_{71} \mathrm{H}_{112} \mathrm{~N}_{16} \mathrm{O}_{16} \mathrm{~S}$ & -1.42 & 5.61 \\
\hline 14 & Unk 1488 & 745.4307 & $\mathrm{C}_{70} \mathrm{H}_{118} \mathrm{~N}_{15} \mathrm{O}_{18} \mathrm{~S}$ & -2.07 & 5.91 \\
\hline 14 & Unk 1490 & 746.4202 & $\mathrm{C}_{72} \mathrm{H}_{114} \mathrm{~N}_{16} \mathrm{O}_{16} \mathrm{~S}$ & -1.69 & 5.56 \\
\hline 14 & Unk 1504 & 753.4296 & $\mathrm{C}_{73} \mathrm{H}_{116} \mathrm{~N}_{16} \mathrm{O}_{16} \mathrm{~S}$ & -1.95 & 5.69 \\
\hline 14 & Unk 1508 & 755.4265 & $\mathrm{C}_{73} \mathrm{H}_{118} \mathrm{~N}_{15} \mathrm{O}_{15} \mathrm{~S}_{2}$ & 0.67 & 5.41 \\
\hline 14 & Unk 1522 & 762.4341 & $\mathrm{C}_{74} \mathrm{H}_{120} \mathrm{~N}_{15} \mathrm{O}_{15} \mathrm{~S}_{2}$ & 0.74 & 5.57 \\
\hline
\end{tabular}
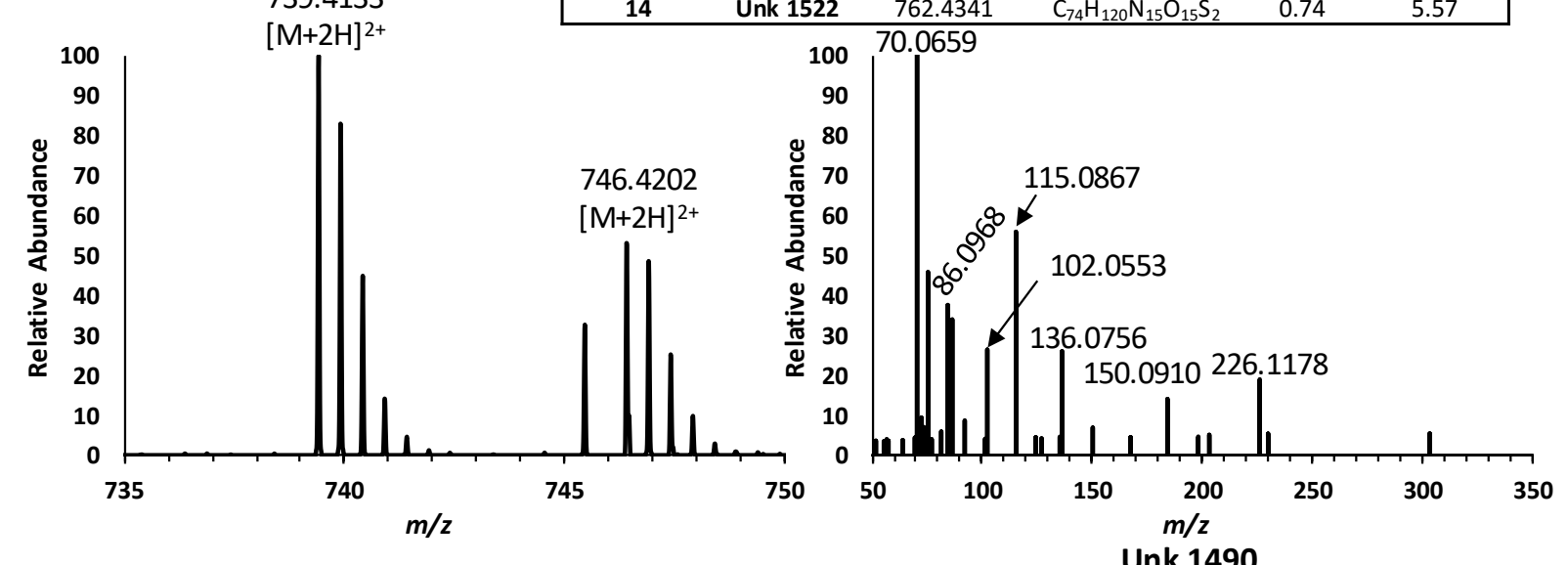

Figure 3.13: The GNPS network cluster \#14 (top right) for an unidentified chemical group with congeners produced by M. aeruginosa CPCC 299 (red) and CPCC 633 (blue). LC-MS analysis of the congeners (top right) with an MS spectrum (bottom left) at 5.65 minutes to show coelution of two congeners. The MS/MS of the congener Unk $1490\left(\mathrm{~m} / z 746.4204[\mathrm{M}+2 \mathrm{H}]^{2+}\right)$, bottom right) with proteogenic immonium ions $m / z 70.0659$ (Pro), 102.0553 (Gln), 136.0756 (Tyr), and the nonproteogenic and potentially diagnostic product ion $\mathrm{m} / \mathrm{z} 115.0865$.

Immonium ions shared between these compounds are $m / z 70.0657$ (Pro), $m / z$ 84.0451 (Arg), $m / z$ 86.0968 (Lxx), $m / z 102.0553$ (Glu), $m / z 136.0755$ (Tyr), and the diagnostic product ion $\mathrm{m} / z$ $115.0865\left(\mathrm{C}_{5} \mathrm{H}_{11} \mathrm{~N}_{2} \mathrm{O}\right)$. This characteristic product ion paired with any of the amino acid immonium ions mentioned could be used to target all congeners with these molecular features.

The LC-MS analysis provides a molecular formula and MS/MS analysis provides information on the building blocks of the metabolite, but connectivity and structure of non-proteogenic amino acids cannot be concluded without other spectroscopic information. Other information that has been derived from this molecular network is the relatedness of the metabolites. For example, in 
the MS spectra of Figure 3.13, the two precursor ions Unk $1476\left(\mathrm{~m} / \mathrm{z} 739.4133[\mathrm{M}+2 \mathrm{H}]^{2+}\right)$ and Unk $1490\left(\mathrm{~m} / z 746.4202[\mathrm{M}+2 \mathrm{H}]^{2+}\right)$, have a mass difference of 7.0051 which equates to a mass difference of 14.0185 when factoring that these molecules are doubly charged. This is the difference of a methyl group, suggesting the variation between these metabolites is a methyl modification. Another example is a mass difference of 7.9956 between Unk $1490(\mathrm{~m} / \mathrm{z} 746.4202$ $\left.[\mathrm{M}+2 \mathrm{H}]^{2+}\right)$ and Unk $1474\left(\mathrm{~m} / z 738.4238[\mathrm{M}+2 \mathrm{H}]^{2+}\right)$, which equates to a molecular mass difference of 15.9970, or 1 oxygen atom suggesting Unk 1490 has substituted a $\mathrm{H}$ for an OH. More information is required to understanding the full structure of these metabolites and their potential toxicity. These will be targets for future chemical characterizations.

\subsubsection{Relative abundance of cyanopeptides produced by Microcystis strains}

The information acquired from untargeted metabolomic techniques such as PCA, loading plot, and molecular networking allows for an understanding of the strain specific metabolites for the investigated Microcystis strains. With limited availability for reference materials of the thousands of cyanopeptide congeners reported, interpretation of the proportions of metabolites has been done here with peak area normalized with dried biomass. This normalization provides a proxy for comparison of the relative proportions of different metabolites and metabolite groups within an extract as well as comparison to other strains. Compounds identified within the molecular network are used here to describe the relative amounts of the secondary metabolites produced by the five studied Microcystis strains (Figure 3.14). 

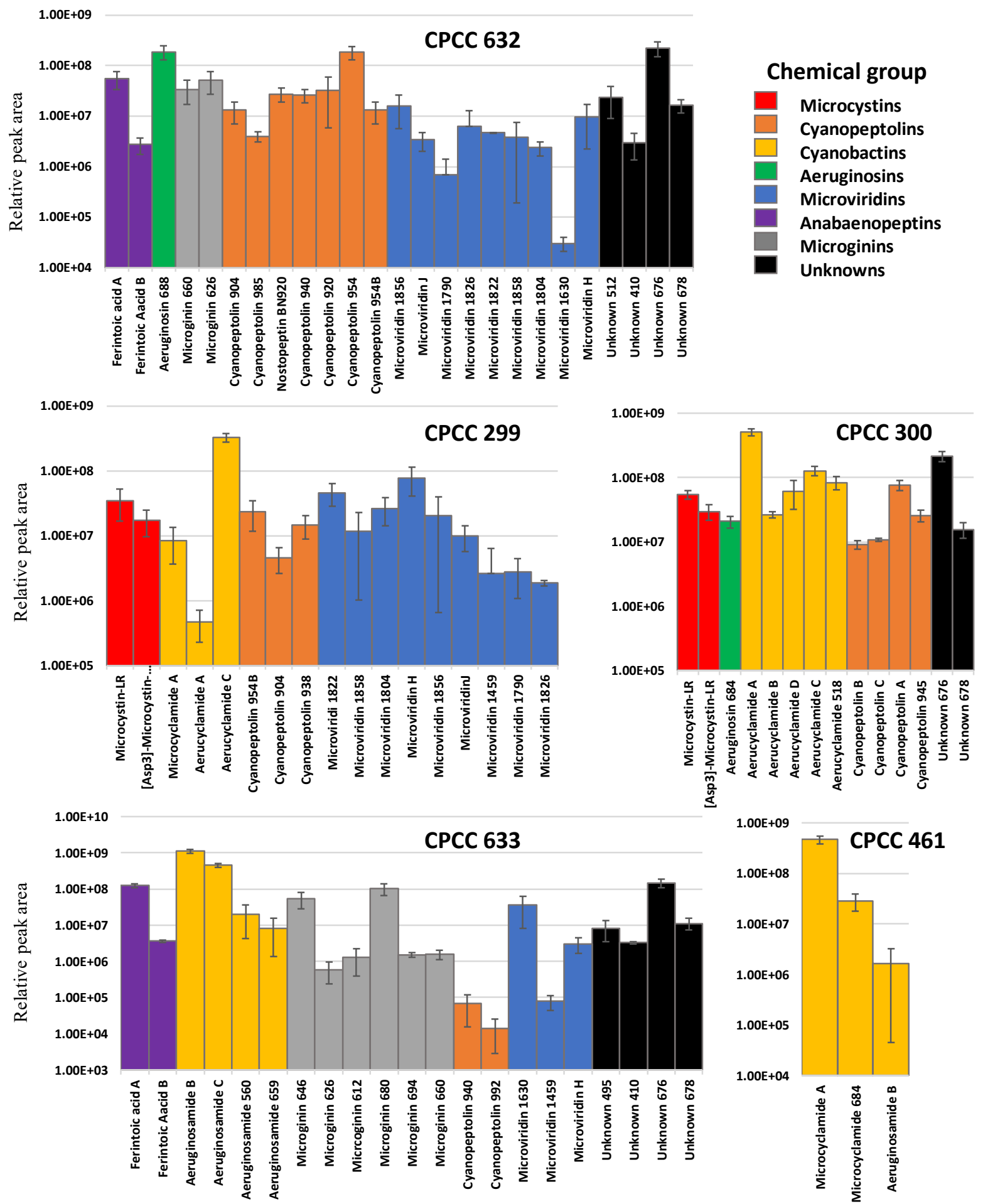

Figure 3.14: Relative abundance of metabolites on day 15 when grown in $\mathrm{BG}-11$ media at $27^{\circ} \mathrm{C}$, generated with normalized peak area of identified secondary metabolites produced by 4 Microcystis aeruginosa strains CPCC 299 (top left), CPCC 300 (middle left), CPCC 632 (bottom left), CPCC 633 (middle right) and 1 Microcystis flos-aquae strain CPCC 461 (bottom right). Metabolites coloured based on cyanopeptide group. 
For all strains that produce cyanobactins (yellow), M. aeruginosa CPCC 299, CPCC 300, and CPCC 633 and M. flos-aquae CPCC 461, these metabolites have the highest relative abundance when comparing to metabolites within the other chemical classes. The MC congeners produced by M. aeruginosa CPCC 299 and CPCC 300 are produced in relatively similar amounts in relation to one another, assuming similar relative responses. For most cyanopeptide groups, there are typically select congeners produced in relatively larger amounts compared to the others. For example, the microginins (grey) MG 646 and MG 680 produced by M. aeruginosa CPCC 633 are in higher proportions than the other microginins. Further, for both M. aeruginosa CPCC 632 and CPCC 633, the anabaenopeptin ferintoic acid A is produced in larger quantities than ferintoic acid B. This information allows for a better understanding at the production of lower abundance metabolites and the importance of sensitive instrumentation for the detection and subsequent identification of all cyanopeptide variants present within an extract.

\subsection{Semi-targeted mass-spectrometry based metabolomics: application to cyanopeptide groups}

Advances in mass spectrometry instrumentation and chemometrics together provide opportunities to better study cyanopeptide diversity. Cyanopeptides and other microbial natural products, are typically biosynthesized as mixtures of structurally related compounds, rather than as a single unique entity. The tandem mass spectrometry (MS/MS) spectra of natural products are highly dependent on their chemical structure, meaning groups of structurally related compounds often generate common product ions or neutral losses. This fact was exploited in some of the untargeted analysis described in section 3.1. Post-acquisition data processing strategies can be used to search MS/MS spectra generated with data dependent acquisition (DDA) experiments for sets of characteristic, or diagnostic, product ions that correspond to specific structural features (Renaud et al. 2015; 2017). Previously, the diagnostic fragmentation filtering (DFF) module within the 
open-source bioinformatics software platform MZmine2 was developed, which facilitates rapid product ion searching with a user-friendly interface (Walsh et al. 2018). DFF can be used to identify all compounds within a group, including known and new compounds useful for dereplication and identifying new targets for chemical characterizations. The peptidyl nature and common structural features that differentiate cyanopeptide groups generates diagnostic product ions, including immonium ions, in their MS/MS spectra that can be targeted by semi-targeted metabolomics approaches such as DFF. For example, the MC chemical group is unified by the presence of the non-proteinaceous amino acid Adda and cyanopeptolins with Ahp (McDonald et al., 2020; McMullin et al., 2019).

This DFF post-acquisition data processing tool uses MZmine2 (http://mzmine.github.io/) to search tandem MS data files for compounds with the diagnostic product ions of interest. The output is a 2-dimensional plot with precursor ion $m / z$ on the x-axis and product ion $m / z$ on the y-axis (for example, Figure 3.16). Each data point within the DFF plot is a product ion, which dictates the $y-$ coordinate, with the exception of intact precursor ions that appear on the diagonal axis. The $\mathrm{x}$ coordinate is based on the intact precursor ion that was selected for fragmentation. What appears on the plot are vertical lines, each corresponding to a metabolite that contains the diagnostic product ions of interest. In some cases, if the metabolites are very structurally similar, there is an appearance of a horizontal line, due to many shared product ions. Other parameters that dictate the specificity of these plots, include $\mathrm{m} / \mathrm{z}$ tolerance, minimum base peak intensity, and precursor ion $\mathrm{m} / \mathrm{z}$ and retention time ranges. Another feature that can be used to increase selectivity towards a particular group of chemicals, is the input of diagnostic neutral losses. For example, the aeruginosin chemical group has many congeners that are sulfated. The loss of the sulfate group is a neutral loss from the precursor ion, can typically be seen with the full MS scan as well as the 
fragmentation spectra. Nonetheless, this can be used as a diagnostic tool for identifying metabolites and thus is useful when considering chemical classes that are sulfated, phosphorylated, etc.

The advantage of having $\mathrm{N}^{15}$-labelled MS and tandem MS data alongside the unlabelled equivalent is the number of nitrogen atoms within the intact precursor ion and any resultant product ions. With this knowledge, conclusion on a molecular formula and additional evidence of a molecule's putative identity. Further, this can be applied to the tandem MS to aid in identifying the structure of the diagnostic product ion.

The results from the untargeted analysis provide insight into product ions and product ion pairs that can be used in a search for congeners of the chemical group in other untargeted data sets. Furthermore, the parameters used to generate the molecular network potentially exclude metabolites that are structurally diverse and unrelated to other metabolites as individual metabolites are excluded. In this section, DFF plots are used to detect all metabolites with specific structural features from cultured strain extracts and the tool shows its capacity to detect cyanopeptides from surface water and bloom samples (Table 3.2). The chemical classes investigated here are those that are well defined: MCs, cyanopeptolins, aeruginosins, and microginins. 
Table 3.2: Collection information for Microcystis strains and environmental surface water and bloom samples.

\begin{tabular}{|c|c|c|c|}
\hline Strain/Sample Name & Sample type & Collection Date & Collection Site \\
\hline CPCC 299 & M. aeruginosa strain & $08 / 90$ & Pretzlaff Pond AB, Canada \\
\hline CPCC 300 & M. aeruginosa strain & $08 / 90$ & Pretzlaff Pond AB, Canada \\
\hline CPCC 461 & M. flos-quae strain & $08 / 75$ & Fox River WI, USA \\
\hline CPCC 632 & M. aeruginosa strain & $10 / 48$ & Lake Mendota WI, USA \\
\hline CPCC 633 & M. aeruginosa strain & $10 / 54$ & Little Rideau Lake, ON Canada \\
\hline$(1)$ & Water & $08 / 19$ & Wildwood Conservation \\
\hline$(2)$ & Water & $08 / 19$ & Scugog Lake \\
\hline$(3)$ & Bloom & $09 / 19$ & Lac Breton \\
\hline$(4)$ & Bloom & $10 / 13$ & Lake Land \\
\hline$(5)$ & Bloom & $06 / 13$ & Lake Heney \\
\hline$(6)$ & Bloom & $06 / 09$ & Lake Land \\
\hline$(7)$ & Bloom & $07 / 13$ & Lake Land \\
\hline$(8)$ & Bloom & $06 / 10$ & Lake Land \\
\hline$(9)$ & Bloom & $06 / 18$ & \\
\hline
\end{tabular}

\subsubsection{Semi-targeted analysis: Microcystins}

The information gathered from the MS/MS of MC standards provided a diagnostic pair of product ions for the Adda moiety ( $\mathrm{m} / \mathrm{z} 135.0803$ and $\mathrm{m} / \mathrm{z} 163.1113$, Figure 3.15) as well as a diagnostic product ion for the non-proteinaceous amino acid Mdha $(\mathrm{m} / \mathrm{z} 213.0865$, Figure 3.15, Table 3.3). The product ions selective for the Adda moiety were then used to screen Microcystis extracts and environmental water samples for the presence of Adda containing metabolites (Figure 3.16). 

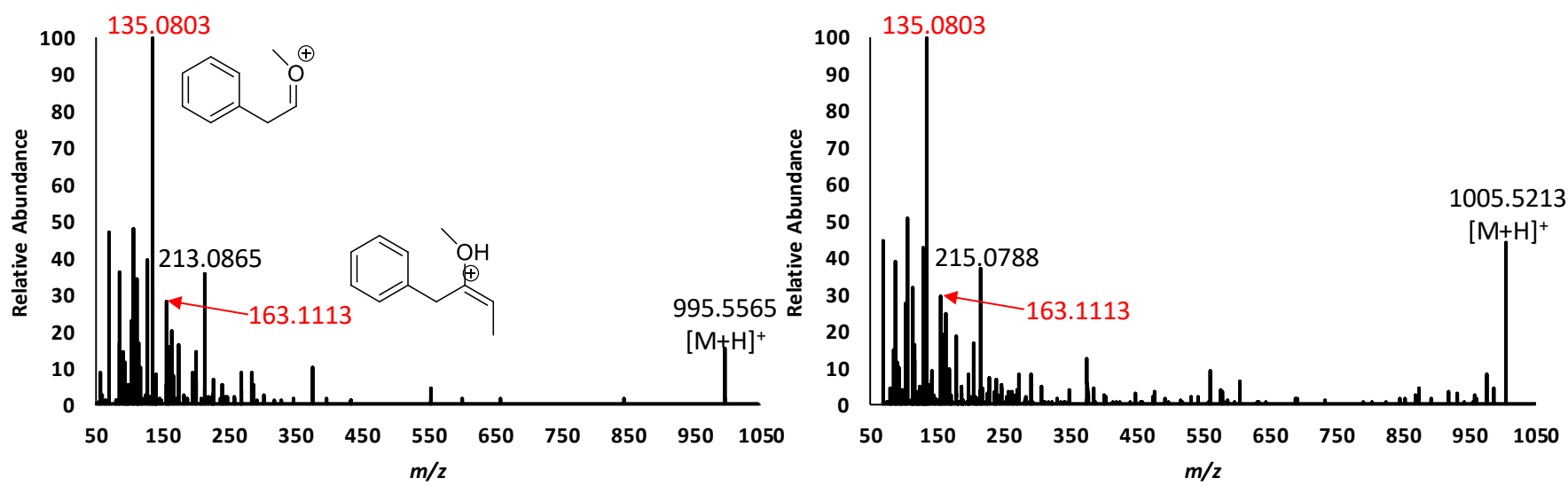

Figure 3.15: MS/MS spectrum of MC-LR $\left.(m / z \text { 995.5565 [M+H] }]^{+}, \mathrm{C}_{49} \mathrm{H}_{74} \mathrm{~N}_{10} \mathrm{O}_{12}\right)$ produced by $M$. aeruginosa CPCC 300 grown in BG-11 media (left) and $\mathrm{NaN}^{15} \mathrm{NO}_{3}$-supplemented $\mathrm{BG}$-11 (right), with representative structures of product ions generated from the Adda moiety, $\mathrm{m} / \mathrm{z} 135.0803$ and $\mathrm{m} / \mathrm{z}$ 163.1113. The molecular ion increases by 10 mass units indicating the incorporation of 10 nitrogen atoms into the microcystin scaffold.

Table 3.3: Product ions specific to the Adda moiety present within metabolites of the MC chemical group.

\begin{tabular}{|c|c|}
\hline Amino acid & Product ion $\boldsymbol{m} / \boldsymbol{z}$ \\
\hline Adda & 135.0803 \\
\hline Adda & 163.1113 \\
\hline Mdha & 213.0865 \\
\hline
\end{tabular}

Untargeted MS based metabolomics showed that two of the five investigated Microcystis strains produce MCs and are considered "toxic", M. aeruginosa CPCC 299 and CPCC 300 (Figure 3.4, (Racine et al., 2019)). Figure 3.16 contains a diagnostic fragmentation filtering (DFF) plot generated with a search for the characteristic product ions from the Adda moiety within the intracellular extract from M. aeruginosa CPCC 300. The series of vertical lines between $\mathrm{m} / \mathrm{z} 950$ 1000 are the three MCs produced by this strain, $\left[\mathrm{Asp}^{3}, \mathrm{Dha}^{7}\right]-\mathrm{MC}-\mathrm{LR},\left[\mathrm{Asp}^{3}\right]-\mathrm{MC}-\mathrm{LR}$, and MCLR (Figure 3.16). The identity of these congeners was determined with a match of high-resolution precursor ion $m / z$, product ion $m / z$, and for [Asp $\left.{ }^{3}\right]-\mathrm{MC}-\mathrm{LR}$ and MC-LR comparison of retention time to a purified standard (Racine et al., 2019). The vertical lines between $\mathrm{m} / \mathrm{z} 450-500$ represent 
the MS/MS spectra generated from the doubly charged precursor ions of [Asp $\left.{ }^{3}\right]-\mathrm{MC}-\mathrm{LR}$ and MCLR.

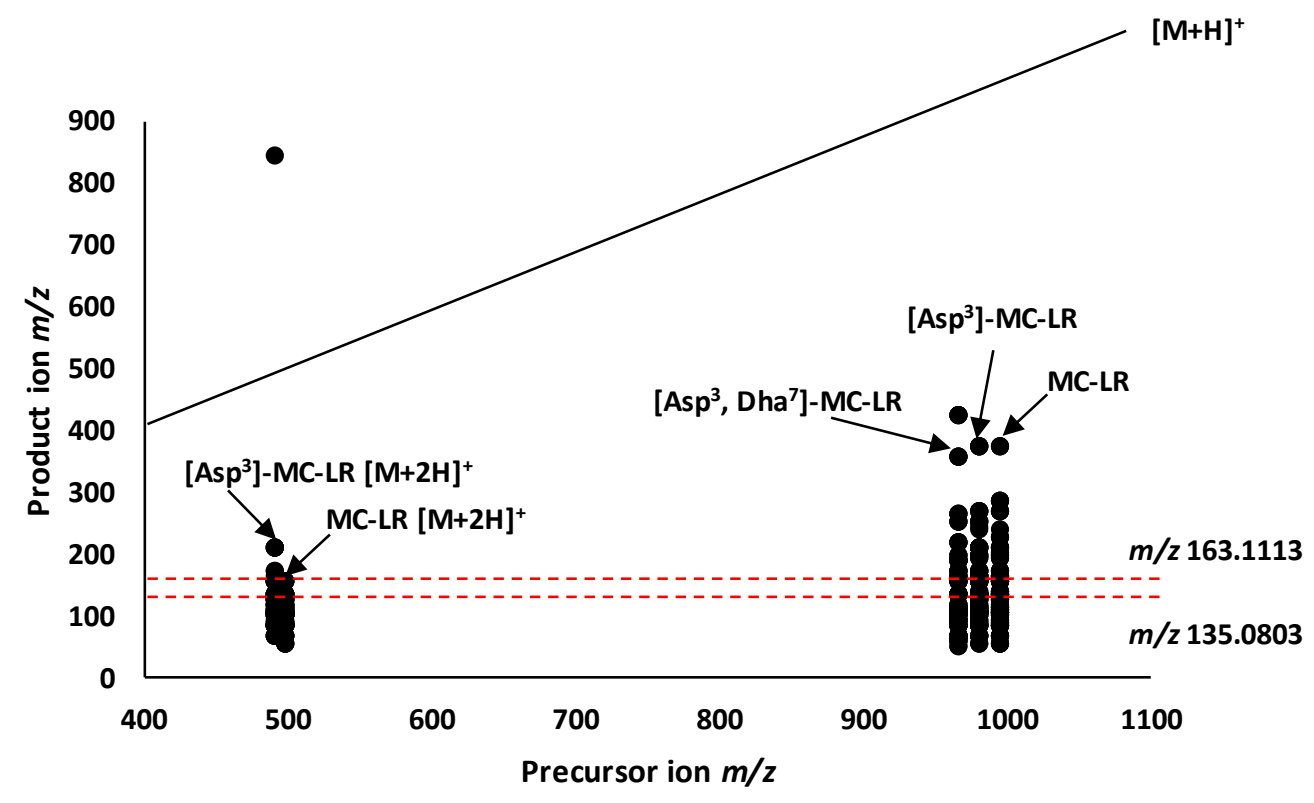

Figure 3.16: Diagnostic fragmentation filter plot targeting Adda-containing metabolites produced by $M$. aeruginosa CPCC 300 searching for the product ions $m / z 135.0803$ and $m / z 163.1113$, characteristic of the Adda moiety.

This tailored method for the detection of MC metabolites was successfully applied to surface water samples and bloom samples. Five MCs were detected within environmental sample \#3, with the identity of three confirmed by comparison to a reference standard (Figure 3.17). The two unknown MCs did not match previously reported MC variants. 


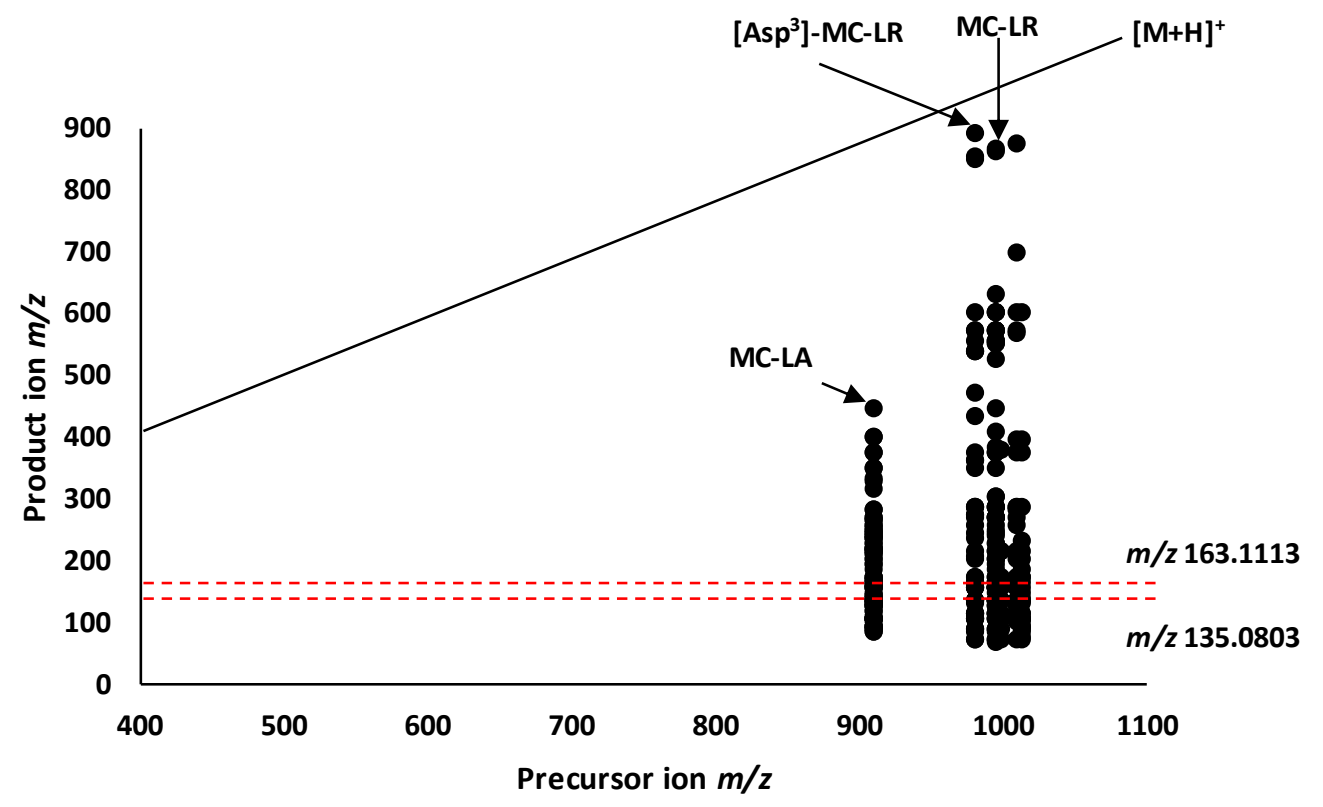

Figure 3.17: Representative diagnostic fragmentation filtering plot targeting Adda-containing metabolites within the environmental sample \#3 searching for the product ions $\mathrm{m} / \mathrm{z} 135.0803$ and $\mathrm{m} / \mathrm{z}$ 163.1113, characteristic of the Adda moiety.

Table 3.4: Microcystins detected by DFF within Microcystis strains and environmental water and bloom samples.

\begin{tabular}{|c|c|c|c|c|c|c|c|c|c|}
\hline \multirow[b]{2}{*}{ Name } & \multirow[b]{2}{*}{$\begin{array}{c}{[\mathbf{M}+\mathbf{H}]^{+}} \\
m / z\end{array}$} & \multirow[b]{2}{*}{$\begin{array}{c}\text { Molecular } \\
\text { formula }\end{array}$} & \multirow[b]{2}{*}{$\begin{array}{l}\text { Error } \\
(\mathrm{ppm})\end{array}$} & \multirow[b]{2}{*}{$\begin{array}{c}\text { RT } \\
\text { (min) }\end{array}$} & \multicolumn{2}{|c|}{$\begin{array}{l}\text { M. aeruginosa } \\
\text { strain }\end{array}$} & \multicolumn{3}{|c|}{ Environmental sample \# } \\
\hline & & & & & $\begin{array}{c}\text { CPCC } \\
299\end{array}$ & $\begin{array}{c}\text { CPCC } \\
300\end{array}$ & 3 & 4 & 5 \\
\hline MC-LR & 995.5565 & $\mathrm{C}_{49} \mathrm{H}_{74} \mathrm{~N}_{10} \mathrm{O}_{12}$ & -1.69 & 3.29 & + & + & + & + & + \\
\hline$\left[\mathrm{Asp}^{3}\right]-\mathrm{MC}-\mathrm{LR}$ & 981.5385 & $\mathrm{C}_{48} \mathrm{H}_{72} \mathrm{~N}_{10} \mathrm{O}_{12}$ & -0.71 & 3.40 & + & + & + & & \\
\hline $\begin{array}{c}{\left[\mathrm{Asp}^{3}, \mathrm{Dha}^{7}\right]-} \\
\mathrm{MC}-\mathrm{LR}\end{array}$ & 967.5254 & $\mathrm{C}_{47} \mathrm{H}_{70} \mathrm{~N}_{10} \mathrm{O}_{12}$ & 1.76 & 3.49 & + & + & & & \\
\hline MC-LA & 910.4923 & $\mathrm{C}_{46} \mathrm{H}_{67} \mathrm{~N}_{7} \mathrm{O}_{12}$ & 0.28 & 5.22 & & & + & & + \\
\hline MC 998 & 999.5635 & & & 3.25 & & & + & & \\
\hline MC 1008 & 1009.5732 & $\mathrm{C}_{50} \mathrm{H}_{76} \mathrm{~N}_{10} \mathrm{O}_{12}$ & 1.49 & 3.41 & & & + & & \\
\hline MC 1012 & 1013.5663 & & & 3.12 & & & + & & \\
\hline
\end{tabular}

\subsubsection{Semi-targeted analysis: Cyanopeptolins}

A comprehensive cyanopeptolin sequence database (>230 variants as of August 2020) was generated to investigate the distribution and frequency of residues at different positions within the cyanopeptolin scaffold (Supp. Table S2). Rapid identification of common and rare partial cyanopeptolin sequences was enabled as well as identification of new and known cyanopeptolin 
congeners. Most variants possess the characteristic Ahp moiety, with approximately $8 \%$ containing the methoxy-Ahp (Amp) moiety at residue $4.14 \%$ of all cyanopeptolin congeners within the compiled database contain the partial sequence NMePhe-Lxx-Ahp, 12\% contain NMeTyr-LxxAhp, $4 \%$ contain NMePhe-Phe-Ahp, and 20\% contain NMeTyr-Phe-Ahp. All cyanopeptolins contain an $\mathrm{NMe}$-aromatic amino acid at residue 2, which generates a characteristic immonium within the MS/MS. Another product ion generated within the MS/MS of cyanopeptolins that provides structural information is the $\left[\text { Residue } 3-\mathrm{Ahp}-\mathrm{H}_{2} \mathrm{O}-\mathrm{CO}\right]^{+}$product ion. These two product ions provide structural information regarding the parent molecule. The identity of these diagnostic product ions was confirmed by comparison to a purified reference material (Cyanopeptolin A which contains NMePhe-Leu-Ahp), followed by ${ }^{15} \mathrm{~N}$-labelling experiments (Figure 3.18). The Microcystis strains were grown in $\mathrm{Na}^{15} \mathrm{NO}_{3}$-supplemented BG-11 media which resulted in incorporation of the isotope within the cyanopeptide scaffold. This allows quantification of the number of nitrogen atoms within a product ion, as the mass difference between the product ion within the unlabelled spectra versus the $\mathrm{N}^{15}$-labelled spectra equates to the number of nitrogen atoms present (Figure 3.18). 

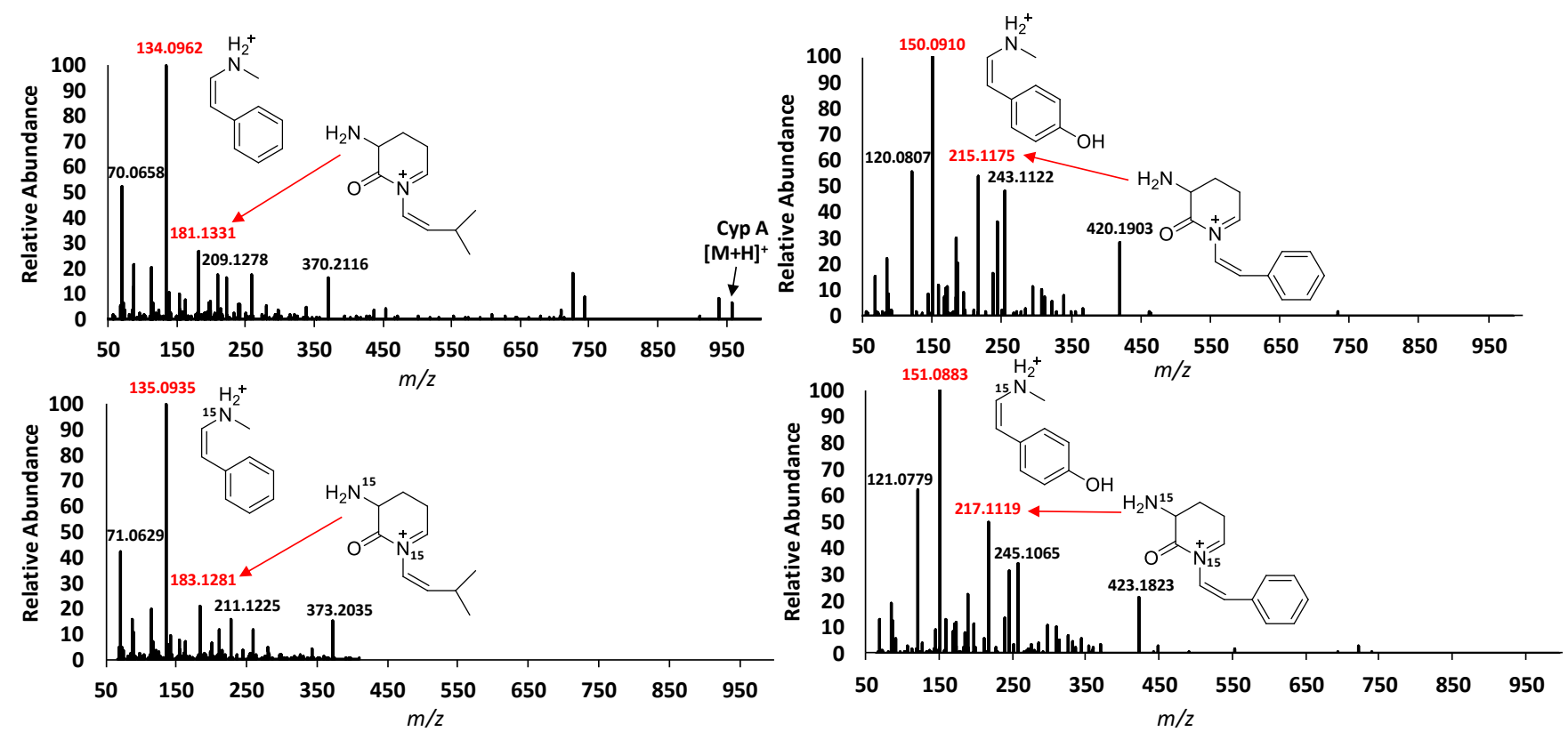

Figure 3.18: 37.5 NCE MS/MS spectra of cyanopeptolin A (left) and nostopeptin BN920 (right) produced by $M$. aeruginosa CPCC 300 and CPCC 632, respectively. MS/MS spectra for each cyanopeptolin are displayed when cyanobacteria were grown in $\mathrm{BG}-11$ (top) and $\mathrm{Na}^{15} \mathrm{NO}_{3}$ supplemented BG-11 (bottom) media. (Top left) Diagnostic product ions for cyanopeptolin A at $\mathrm{m} / \mathrm{z} 134.0963$ and 181.1331 correspond to $\mathrm{MePhe}$ at position 2 and Leu-Ahp at positions 3 and 4, respectively. (Bottom left) Incorporation of ${ }^{15} \mathrm{~N}$ into peptide scaffolds supports these diagnostic product ions. Corresponding $\mathrm{MePhe}$ and Leu-Ahp product ions are observed at $\mathrm{m} / \mathrm{z} 135.0935$ and 183.1281. (Top right) Diagnostic product ions for nostopeptin BN920 at $\mathrm{m} / z 150.0912$ and 215.1183 correspond to MeTyr at position 2, and Leu-Ahp at positions 3 and 4, respectively. (Bottom right) Incorporation of ${ }^{15} \mathrm{~N}$ into peptide scaffolds supports these product ions observed at 151.0883 and 217.1119, that contain 1 and 2 nitrogen atoms, respectively.

This diagnostic information regarding the partial amino acid sequence and the frequency within cyanopeptolin congeners, allows for rapid identification and classification of the metabolite. With a semi-targeted approach, all cyanopeptolins within a Microcystis extract can be identified by searching MS/MS datasets for pairs of abundant product ions corresponding to specific partial amino acid sequences (Table 3.5). 
Table 3.5: Product ions specific to the partial amino acid sequences present within metabolites of the cyanopeptolin chemical class.

\begin{tabular}{|c|c|c|c|c|}
\hline Partial sequence & $\begin{array}{c}\text { Residue 2 } \\
\text { immonium ion }\end{array}$ & $\begin{array}{c}\boldsymbol{m} / \boldsymbol{z} \\
{[\text { Residue 3-Ahp- }} \\
\text { H2O-CO] }^{+} \\
\text {fragment }\end{array}$ & $\boldsymbol{m} / \mathbf{z}$ \\
\hline NMePhe-Lxx-Ahp & NMePhe & 134.0963 & Lxx-Ahp & 181.1331 \\
\hline NMeTyr-Lxx-Ahp & NMeTyr & 150.0912 & Lxx-Ahp & 181.1331 \\
\hline NMePhe-Phe-Ahp & NMePhe & 134.0963 & Phe-Ahp & 215.1183 \\
\hline NMeTyr-Phe-Ahp & NMeTyr & 150.0912 & Phe-Ahp & 215.1183 \\
\hline NMe-Cl-Tyr-Lxx-Ahp & NMe-Cl-Tyr & 184.0523 & Lxx-Ahp & 181.1331 \\
\hline NMe-Cl-Tyr-Phe-Ahp & NMe-Cl-Tyr & 184.0523 & Phe-Ahp & 215.1183 \\
\hline
\end{tabular}

In this study, it was determined that four of the five investigated Microcystis strains produced cyanopeptolins using the product ion information collected regarding the partial amino acid sequences. The data generated with this semi-targeted approach agrees with the untargeted data generated via the GNPS molecular networking (Figure 3.3). The four M. aeruginosa strains (CPCC 299, CPCC 300, CPCC 632, and CPCC 633) produce different mixtures of cyanopeptolin congeners with varying partial amino acid sequences (Table 3.6). M. aeruginosa CPCC 300 is the only strain to product congeners with the partial sequence NMePhe-Lxx-Ahp, including previously reported congeners cyanopeptolins A-C and cyanopeptolin 963A (Cornel et al., 1993, Bister et al., 2004). M. aeruginosa CPCC 299 and CPCC 633 produced cyanopeptolins with the exclusive partial amino acid NMePhe-Phe-Ahp. M. aeruginosa CPCC 632 produces congeners containing the partial amino acid sequence NMeTyr-Phe-Ahp and NMe-Cl-Tyr-Phe-Ahp, including nostopeptin BN920 and the chlorinated counterpart cyanopeptolin 954 (Figure 3.19, Von Elert et al., 2005). 


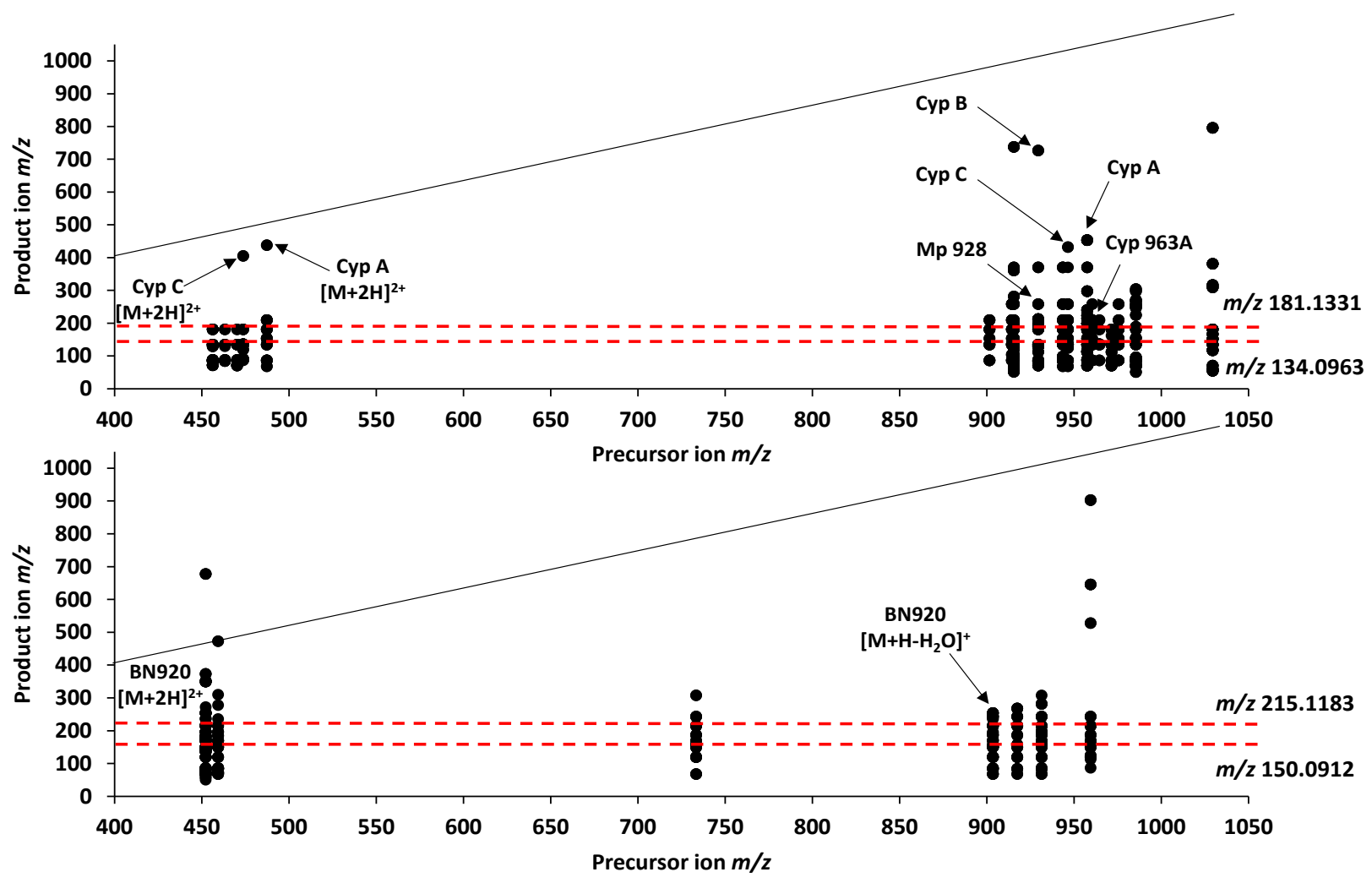

Figure 3.19: Representative DFF plots for cyanopeptolins containing NMe-Phe-Lxx produced by $M$. aeruginosa CPCC 300 (top) and NMe-Tyr-Phe containing cyanopeptolins produced by $M$. aeruginosa CPCC 632 (bottom). Red horizontal lines are indicative of diagnostic product ions required to be considered cyanopeptolins with designated residues at position 2 and 3 . The black solid line represents $[\mathrm{M}+\mathrm{H}]^{+}$or $\left[\mathrm{M}+\mathrm{H}-\mathrm{H}_{2} \mathrm{O}\right]^{+}$precursor ions as designated in Table 2. Each vertical dot represents product ions detected within the MS/MS spectra of corresponding cyanopeptolin precursor ions.

The tailored semi-targeted approach for identification of cyanopeptolin congeners was applied to surface water and bloom samples collected in the National Capital Region and the Greater Toronto Area (Table 3.2). The samples were screened for cyanopeptolins with a search of all MS/MS spectra for product ion pairs relating to the different partial amino acid sequences that are possible. Fifteen cyanopeptolins, including eight new variants, were detected from the investigated environmental samples and each contained different cyanopeptolin profiles (Figure 3.20, Table 3.7). The cyanopeptolin profiles for the investigated environmental samples are different 
suggesting multiple populations of cyanobacteria were residing within the samples water. Previously reported cyanopeptolins that were detected include cyanopeptolin S, cyanopeptolin 972C, micropeptin MZ845, and cyanopeptolin 1013 (McDonald et al., 2020). Cyanopeptolin 954 and the de-chloro version, nostopeptin BN920 were found in both the environmental samples as well as the Microcystis extracts (Figure 3.5, Figure 3.20). Of the eight new variants, one variant was detected and is believed to be the smallest cyanopeptolin reported to date with a $\mathrm{m} / \mathrm{z} 733.3880$ $\left[\mathrm{M}+\mathrm{H}-\mathrm{H}_{2} \mathrm{O}\right]^{+}$. Many important bloom-forming genera including Microcystis, Planktothrix, Dolichospermum, and Woronichinia can all biosynthesize cyanopeptolins resulting in complex cyanopeptolin mixtures with a wide variety of partial amino acid sequences (McDonald et al., 2020).
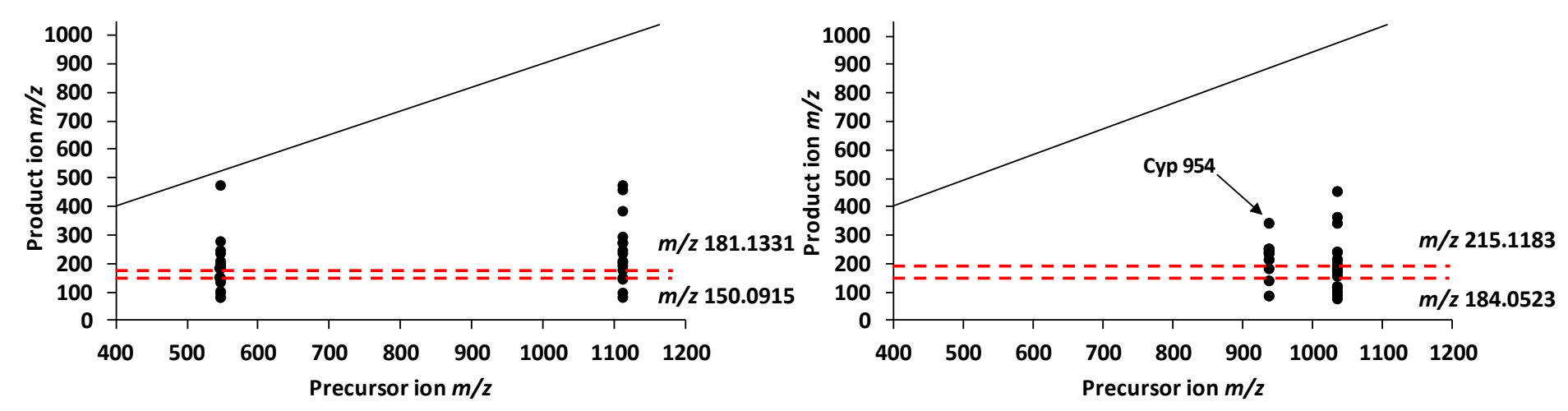

Figure 3.20: Representative cyanopeptolin DFF plots for water samples 3 (left) and 4 (right). DFF plots for cyanopeptolins containing the partial sequence NMe-Tyr-Lxx-Ahp (left) and the partial sequence Cl-NMe-Tyr-Phe-Ahp (right). 
Table 3.6: Cyanopeptolins detected by DFF from Microcystis aeruginosa strains and bloom samples.

\begin{tabular}{|c|c|c|c|c|c|c|c|c|c|c|c|c|c|c|c|}
\hline \multirow{2}{*}{ Name } & \multirow[b]{2}{*}{$\begin{array}{c}\mathbf{M}+\mathbf{H}]^{+} \\
m / z\end{array}$} & \multirow[b]{2}{*}{$\begin{array}{c}\text { Molecular } \\
\text { formula }\end{array}$} & \multirow[b]{2}{*}{$\begin{array}{l}\text { Error } \\
(\mathrm{ppm})\end{array}$} & \multirow[b]{2}{*}{$\begin{array}{c}\mathbf{R t} \\
(\mathbf{m i n})\end{array}$} & \multirow[b]{2}{*}{$\begin{array}{c}\text { Residue } \\
2 \\
\end{array}$} & \multirow[b]{2}{*}{$\begin{array}{c}\text { Residue } \\
\mathbf{3} \\
\end{array}$} & \multicolumn{4}{|c|}{ M. aeruginosa strain } & \multicolumn{4}{|c|}{$\begin{array}{c}\text { Environmental } \\
\text { sample \# }\end{array}$} & \multirow[b]{2}{*}{ Reference } \\
\hline & & & & & & & $\begin{array}{c}\text { CPCC } \\
299\end{array}$ & $\begin{array}{c}\text { CPCC } \\
300\end{array}$ & $\begin{array}{c}\text { CPCC } \\
632\end{array}$ & $\begin{array}{c}\text { CPCC } \\
633\end{array}$ & (3) & (4) & (6) & (7) & \\
\hline Сур 954B & $\begin{array}{c}937.4441 \\
{[\mathrm{M}+\mathrm{H}-} \\
\left.\mathrm{H}_{2} \mathrm{O}\right]^{+}\end{array}$ & $\mathrm{C}_{49} \mathrm{H}_{62} \mathrm{~N}_{8} \mathrm{O}_{12}$ & -1.41 & 3.92 & $\begin{array}{l}\text { NMe- } \\
\text { Phe }\end{array}$ & Phe & + & & & & & & & & This study \\
\hline Сур 954C & $\begin{array}{c}955.4556 \\
{[\mathrm{M}+\mathrm{H}]^{+}}\end{array}$ & $\mathrm{C}_{49} \mathrm{H}_{62} \mathrm{~N}_{8} \mathrm{O}_{12}$ & -0.41 & 3.31 & $\begin{array}{l}\text { NMe- } \\
\text { Phe }\end{array}$ & Phe & + & & & & & & & & This study \\
\hline Сур 938 & $\begin{array}{c}921.4494 \\
{[\mathrm{M}+\mathrm{H}-} \\
\left.\mathrm{H}_{2} \mathrm{O}\right]^{+}\end{array}$ & $\mathrm{C}_{49} \mathrm{H}_{62} \mathrm{~N}_{8} \mathrm{O}_{11}$ & -1.21 & 4.35 & $\begin{array}{l}\text { NMe- } \\
\text { Phe }\end{array}$ & Phe & + & & & & & & & & This study \\
\hline Cyp 922 & $\begin{array}{c}905.4213 \\
{[\mathrm{M}+\mathrm{H}-} \\
\left.\mathrm{H}_{2} \mathrm{O}\right]^{+}\end{array}$ & $\mathrm{C}_{48} \mathrm{H}_{58} \mathrm{~N}_{8} \mathrm{O}_{11}$ & 1.94 & 3.99 & $\begin{array}{l}\text { NMe- } \\
\text { Phe }\end{array}$ & Phe & + & & & & & & & & This study \\
\hline Сур 904 & $\begin{array}{c}887.4652 \\
{[\mathrm{M}+\mathrm{H}-} \\
\left.\mathrm{H}_{2} \mathrm{O}\right]^{+} \\
\end{array}$ & $\mathrm{C}_{46} \mathrm{H}_{64} \mathrm{~N}_{8} \mathrm{O}_{11}$ & -1.08 & 4.16 & $\begin{array}{l}\text { NMe- } \\
\text { Phe }\end{array}$ & Phe & + & & + & & & & & & This study \\
\hline Cyp 918 & $\begin{array}{c}901.4815 \\
{[\mathrm{M}+\mathrm{H}-} \\
\left.\mathrm{H}_{2} \mathrm{O}\right]^{+}\end{array}$ & $\mathrm{C}_{47} \mathrm{H}_{66} \mathrm{~N}_{8} \mathrm{O}_{11}$ & -0.35 & 4.48 & $\begin{array}{l}\text { NMe- } \\
\text { Phe }\end{array}$ & Phe & + & & & + & & & & & This study \\
\hline $\begin{array}{c}\text { Cyanopeptolin } \\
\text { A }\end{array}$ & $\begin{array}{c}957.5391 \\
{[\mathrm{M}+\mathrm{H}]^{+}}\end{array}$ & $\mathrm{C}_{46} \mathrm{H}_{72} \mathrm{~N}_{10} \mathrm{O}_{12}$ & -1.35 & 3.39 & $\begin{array}{l}\text { NMe- } \\
\text { Phe }\end{array}$ & Leu & & + & & & & & & & $\begin{array}{c}\text { Martin et } \\
\text { al. } 1993\end{array}$ \\
\hline $\begin{array}{c}\text { Cyanopeptolin } \\
\text { B }\end{array}$ & $\begin{array}{c}929.5327 \\
{[\mathrm{M}+\mathrm{H}]^{+}}\end{array}$ & $\mathrm{C}_{46} \mathrm{H}_{72} \mathrm{~N}_{8} \mathrm{O}_{12}$ & -1.66 & 3.32 & $\begin{array}{l}\text { NMe- } \\
\text { Phe }\end{array}$ & Leu & & + & & & & & & & $\begin{array}{c}\text { Martin et } \\
\text { al. } 1993\end{array}$ \\
\hline $\begin{array}{c}\text { Cyanopeptolin } \\
\text { C }\end{array}$ & $\begin{array}{c}943.5481 \\
{[\mathrm{M}+\mathrm{H}]^{+}}\end{array}$ & $\mathrm{C}_{47} \mathrm{H}_{74} \mathrm{~N}_{8} \mathrm{O}_{12}$ & -1.90 & 3.72 & $\begin{array}{l}\text { NMe- } \\
\text { Phe }\end{array}$ & Leu & & + & & & & & & & $\begin{array}{c}\text { Martin et } \\
\text { al. } 1993\end{array}$ \\
\hline Сур 900 & $\begin{array}{c}901.5031 \\
{[\mathrm{M}+\mathrm{H}]^{+}}\end{array}$ & $\mathrm{C}_{44} \mathrm{H}_{68} \mathrm{~N}_{8} \mathrm{O}_{12}$ & 0.17 & 3.16 & $\begin{array}{l}\text { NMe- } \\
\text { Phe }\end{array}$ & Lxx & & + & & & & & & & This study \\
\hline Cyp 914 & $\begin{array}{c}915.5178 \\
{[\mathrm{M}+\mathrm{H}]^{+}} \\
\end{array}$ & $\mathrm{C}_{45} \mathrm{H}_{70} \mathrm{~N}_{8} \mathrm{O}_{12}$ & -0.86 & 3.18 & $\begin{array}{l}\text { NMe- } \\
\text { Phe }\end{array}$ & Lxx & & + & & & & & & & This study \\
\hline Сур 974 & $\begin{array}{c}975.5527 \\
{[\mathrm{M}+\mathrm{H}]^{+}}\end{array}$ & $\mathrm{C}_{46} \mathrm{H}_{72} \mathrm{~N}_{10} \mathrm{O}_{13}$ & 1.75 & 4.11 & $\begin{array}{l}\text { NMe- } \\
\text { Phe }\end{array}$ & Lxx & & + & & & & & & & This study \\
\hline Суо 945 & $\begin{array}{c}946.4917 \\
{[\mathrm{M}+\mathrm{H}]^{+}}\end{array}$ & $\mathrm{C}_{49} \mathrm{H}_{67} \mathrm{~N}_{7} \mathrm{O}_{12}$ & -0.36 & 6.04 & $\begin{array}{l}\text { NMe- } \\
\text { Phe }\end{array}$ & Lxx & & + & & & & & & & This study \\
\hline Сур 970 & $\begin{array}{c}971.5565 \\
{[\mathrm{M}+\mathrm{H}]^{+}}\end{array}$ & $\mathrm{C}_{47} \mathrm{H}_{74} \mathrm{~N}_{10} \mathrm{O}_{12}$ & 0.46 & 4.15 & $\begin{array}{l}\text { NMe- } \\
\text { Phe }\end{array}$ & Lxx & & + & & & & & & & This study \\
\hline Cyp 1028 & $\begin{array}{c}1029.5631 \\
{[\mathrm{M}+\mathrm{H}]^{+}}\end{array}$ & $\mathrm{C}_{49} \mathrm{H}_{76} \mathrm{~N}_{10} \mathrm{O}_{14}$ & 1.54 & 4.08 & $\begin{array}{l}\text { NMe- } \\
\text { Phe }\end{array}$ & Lxx & & + & & & & & & & This study \\
\hline $\begin{array}{c}\text { Cyanopeptolin } \\
963 \mathrm{~A}\end{array}$ & $\begin{array}{c}964.5040 \\
{[\mathrm{M}+\mathrm{H}]^{+}}\end{array}$ & $\mathrm{C}_{49} \mathrm{H}_{69} \mathrm{~N}_{7} \mathrm{O}_{13}$ & 1.53 & 5.53 & $\begin{array}{l}\text { NMe- } \\
\text { Phe }\end{array}$ & Leu & & + & & & & & & & $\begin{array}{l}\text { Bister et } \\
\text { al. } 2004\end{array}$ \\
\hline Сур 913 & $\begin{array}{c}914.4673 \\
{[\mathrm{M}+\mathrm{H}]^{+}}\end{array}$ & $\mathrm{C}_{48} \mathrm{H}_{63} \mathrm{~N}_{7} \mathrm{O}_{11}$ & 1.59 & 5.53 & $\begin{array}{l}\text { NMe- } \\
\text { Phe }\end{array}$ & Lxx & & + & & & & & & & This study \\
\hline Сур 977 & $\begin{array}{c}960.5074 \\
{[\mathrm{M}+\mathrm{H}-} \\
\left.\mathrm{H}_{2} \mathrm{O}\right]^{+}\end{array}$ & $\mathrm{C}_{50} \mathrm{H}_{69} \mathrm{~N}_{7} \mathrm{O}_{12}$ & -0.45 & 5.58 & $\begin{array}{l}\text { NMe- } \\
\text { Phe }\end{array}$ & Lxx & & + & & & & & & & This study \\
\hline Сур 984 & $\begin{array}{c}985.5724 \\
{[\mathrm{M}+\mathrm{H}]^{+}}\end{array}$ & $\mathrm{C}_{48} \mathrm{H}_{76} \mathrm{~N}_{10} \mathrm{O}_{12}$ & 0.76 & 4.61 & $\begin{array}{l}\text { NMe- } \\
\text { Phe }\end{array}$ & Lxx & & + & & & & & & & This study \\
\hline $\begin{array}{l}\text { Micropeptin } \\
\text { KB928 }\end{array}$ & $\begin{array}{c}929.5091 \\
{[\mathrm{M}+\mathrm{H}]^{+}}\end{array}$ & $\mathrm{C}_{44} \mathrm{H}_{68} \mathrm{~N}_{10} \mathrm{O}_{12}$ & 0.01 & 3.28 & $\begin{array}{l}\text { NMe- } \\
\text { Phe }\end{array}$ & Ile & & + & & & & & & & $\begin{array}{c}\text { Elkobi- } \\
\text { Peer \& } \\
\text { Carmeli, } \\
2015\end{array}$ \\
\hline $\begin{array}{l}\text { Nostopeptin } \\
\text { BN920 }\end{array}$ & $\begin{array}{c}903.4587 \\
{[\mathrm{M}+\mathrm{H}-} \\
\left.\mathrm{H}_{2} \mathrm{O}\right]^{+}\end{array}$ & $\mathrm{C}_{46} \mathrm{H}_{64} \mathrm{~N}_{8} \mathrm{O}_{12}$ & 0.10 & 3.25 & $\begin{array}{c}\mathrm{NMe-} \\
\mathrm{Tyr}\end{array}$ & Phe & & & + & & & + & & & $\begin{array}{l}\text { von Elert } \\
\text { et al. } 2005\end{array}$ \\
\hline Сур 934 & $\begin{array}{c}917.4752 \\
{[\mathrm{M}+\mathrm{H}-} \\
\left.\mathrm{H}_{2} \mathrm{O}\right]^{+} \\
\end{array}$ & $\mathrm{C}_{47} \mathrm{H}_{66} \mathrm{~N}_{8} \mathrm{O}_{12}$ & -0.91 & 3.43 & $\begin{array}{c}\mathrm{NMe}- \\
\mathrm{Tyr}\end{array}$ & Phe & & & + & & & & & & This study \\
\hline Cyp 948 & $\begin{array}{c}931.4928 \\
{[\mathrm{M}+\mathrm{H}-} \\
\left.\mathrm{H}_{2} \mathrm{O}\right]^{+}\end{array}$ & $\mathrm{C}_{48} \mathrm{H}_{68} \mathrm{~N}_{8} \mathrm{O}_{12}$ & 0.51 & 3.68 & $\begin{array}{l}\mathrm{NMe}- \\
\mathrm{Tyr}\end{array}$ & Phe & & & + & & & & & & This study \\
\hline Сур 976 & $\begin{array}{c}959.5237 \\
{[\mathrm{M}+\mathrm{H}-} \\
\left.\mathrm{H}_{2} \mathrm{O}\right]^{+} \\
\end{array}$ & $\mathrm{C}_{50} \mathrm{H}_{72} \mathrm{~N}_{8} \mathrm{O}_{12}$ & 0.01 & 4.58 & $\begin{array}{l}\text { NMe- } \\
\text { Tyr }\end{array}$ & Phe & & & + & & & + & & & This study \\
\hline
\end{tabular}




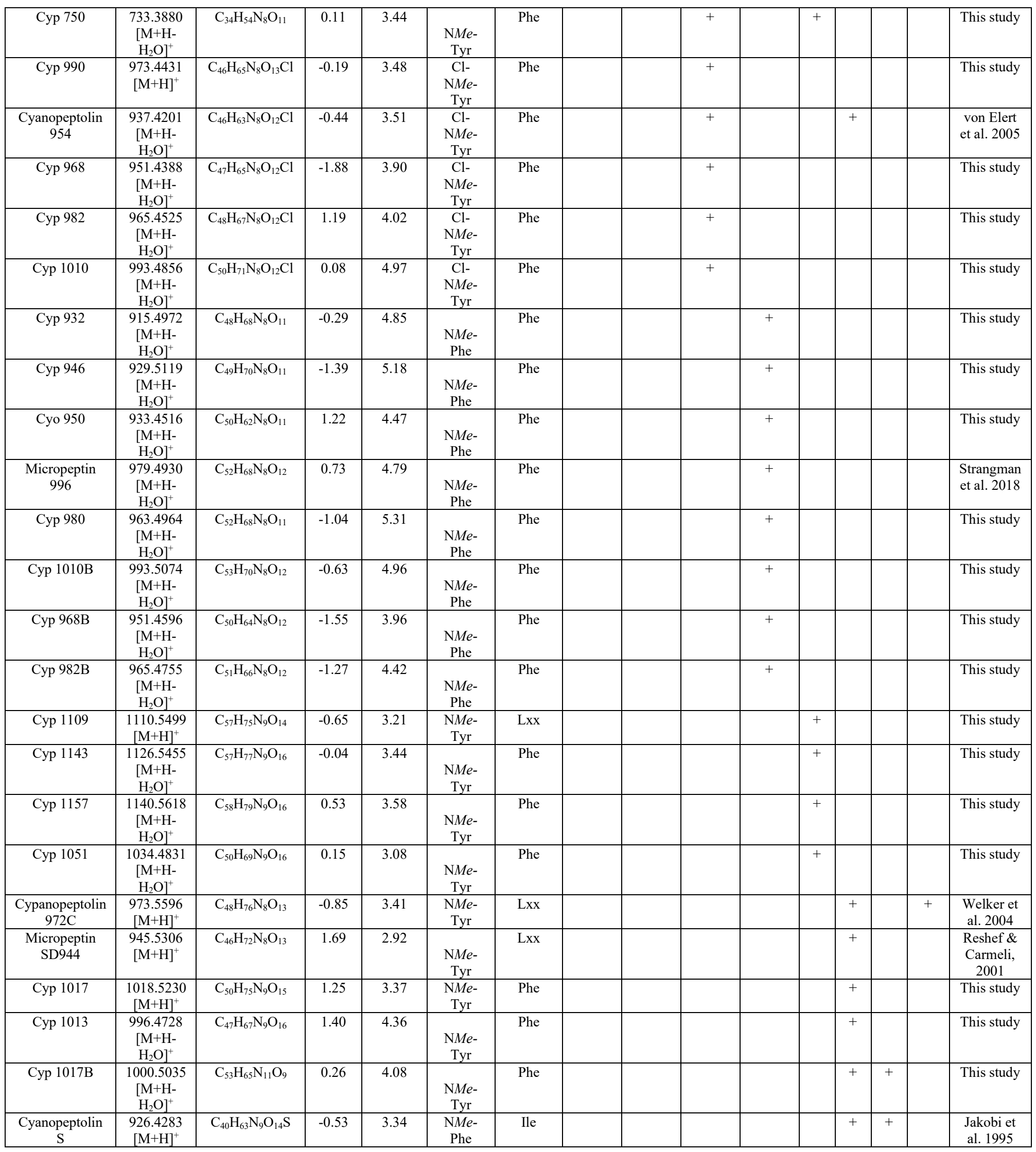




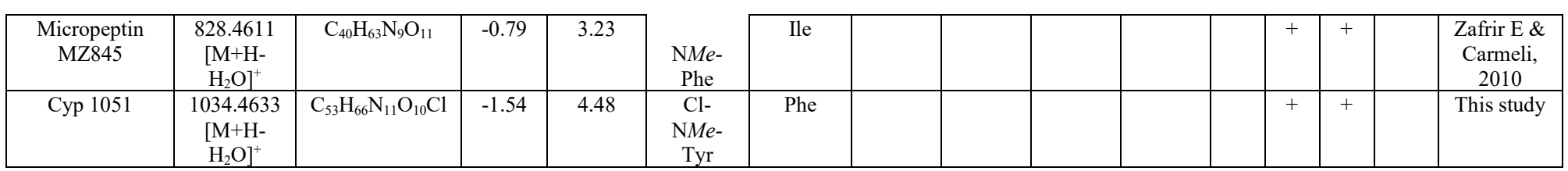

\subsubsection{Semi-targeted analysis: Aeruginosins}

The aeruginosin chemical group is characterized by the presence of the Choi functionality. Diagnostic product ions are generated from the moiety, $[\mathrm{Choi}+\mathrm{H}]^{+} \mathrm{m} / 2 \mathrm{z} 140.1066$ and $\left[\mathrm{Choi}-\mathrm{H}_{2} \mathrm{O}\right.$ $+\mathrm{H}]^{+}$at $\mathrm{m} / \mathrm{z} 122.0964$ (Table 3.7, Figure 3.21, (Welker et al., 2006)). The structure of these diagnostic product ions was confirmed with $\mathrm{N}^{15}$-labelling experiments (Figure 3.21).
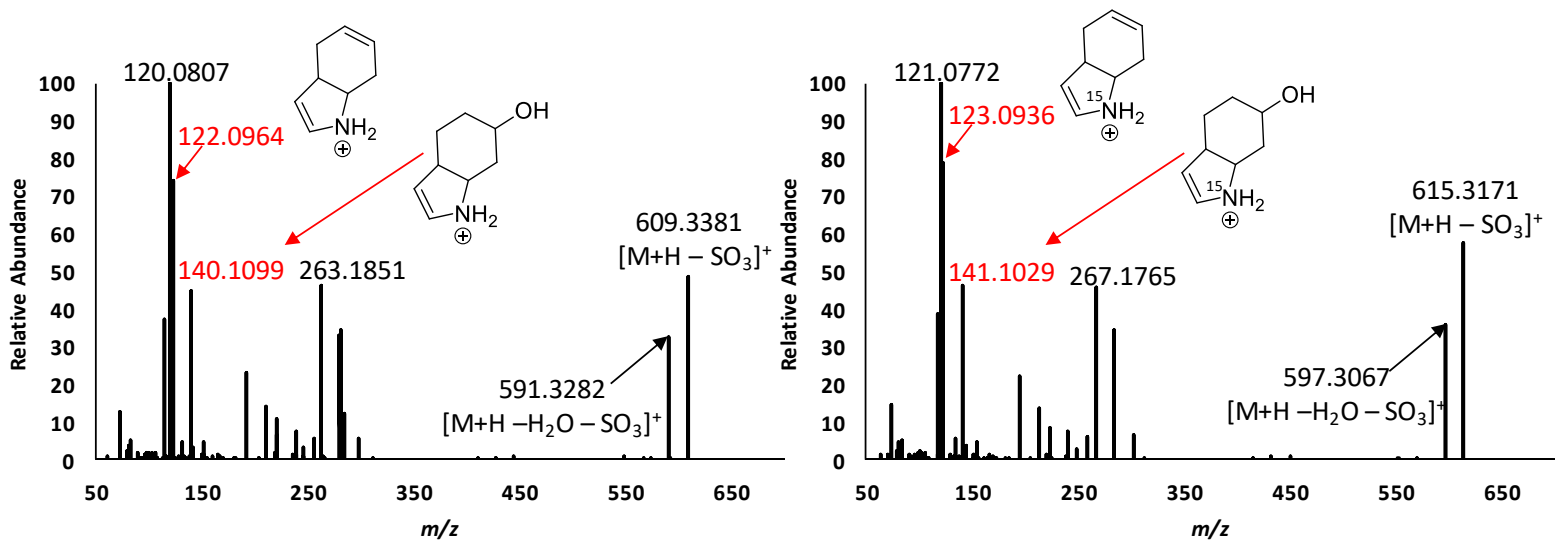

Figure 3.21: MS/MS spectra of aeruginosin 688 produced by M. aeruginosa CPCC 300. MS/MS spectra for the aeruginosin congener are displayed when cyanobacteria were grown in BG-11 (left) and $\mathrm{Na}^{15} \mathrm{NO}_{3}$ supplemented BG-11 (right) media. (Left) Diagnostic product ions for aeruginosin $688\left(\mathrm{~m} / z 689.2958[\mathrm{M}+\mathrm{H}]^{+} ; \mathrm{C}_{32} \mathrm{H}_{44} \mathrm{~N}_{6} \mathrm{O}_{9} \mathrm{~S}\right)$ at $\mathrm{m} / z \mathrm{z} 140.1099$ and $\mathrm{m} / z 122.0964$ correspond to the $[\mathrm{Choi}+\mathrm{H}]^{+}$and $\left[\mathrm{Choi}-\mathrm{H}_{2} \mathrm{O}+\mathrm{H}\right]^{+}$, respectively. Incorporation of ${ }^{15} \mathrm{~N}$ into peptide scaffolds supports these diagnostic product ions. Corresponding [Choi $+\mathrm{H}]^{+}$and $\left[\mathrm{Choi}-\mathrm{H}_{2} \mathrm{O}+\mathrm{H}\right]^{+}$product ions are observed at $\mathrm{m} / \mathrm{z} 141.1029$ and $\mathrm{m} / z 123.0936$ that contain 1 nitrogen atom each.

Table 3.7: Product ions specific to the Choi moiety present within metabolites of the aeruginosin chemical class.

\begin{tabular}{|c|c|}
\hline Moiety & Product ion $\mathbf{m} / \mathbf{z}$ \\
\hline Choi & 140.1066 \\
\hline Choi- $\mathrm{H}_{2} \mathrm{O}$ & 122.0964 \\
\hline
\end{tabular}


A second diagnostic feature for aeruginosins is the occurrence of sulfated congeners. Sulfonation is a common chemical modification that occurs on the Choi moiety in many aeruginosins (Welker et al., 2006). If the sulfated and de-sulfated counterpart had the same retention time, it was assumed that this was generated as an in-source fragment and indicates the organism only produces the sulfated metabolite. If the retention times were different, it was assumed that both metabolites were present within the extract. Based on the molecular network, M. aeruginosa CPCC 300 and CPCC 632 were screened as aeruginosin producers with the DFF approach for any outstanding metabolites containing the Choi functionality.

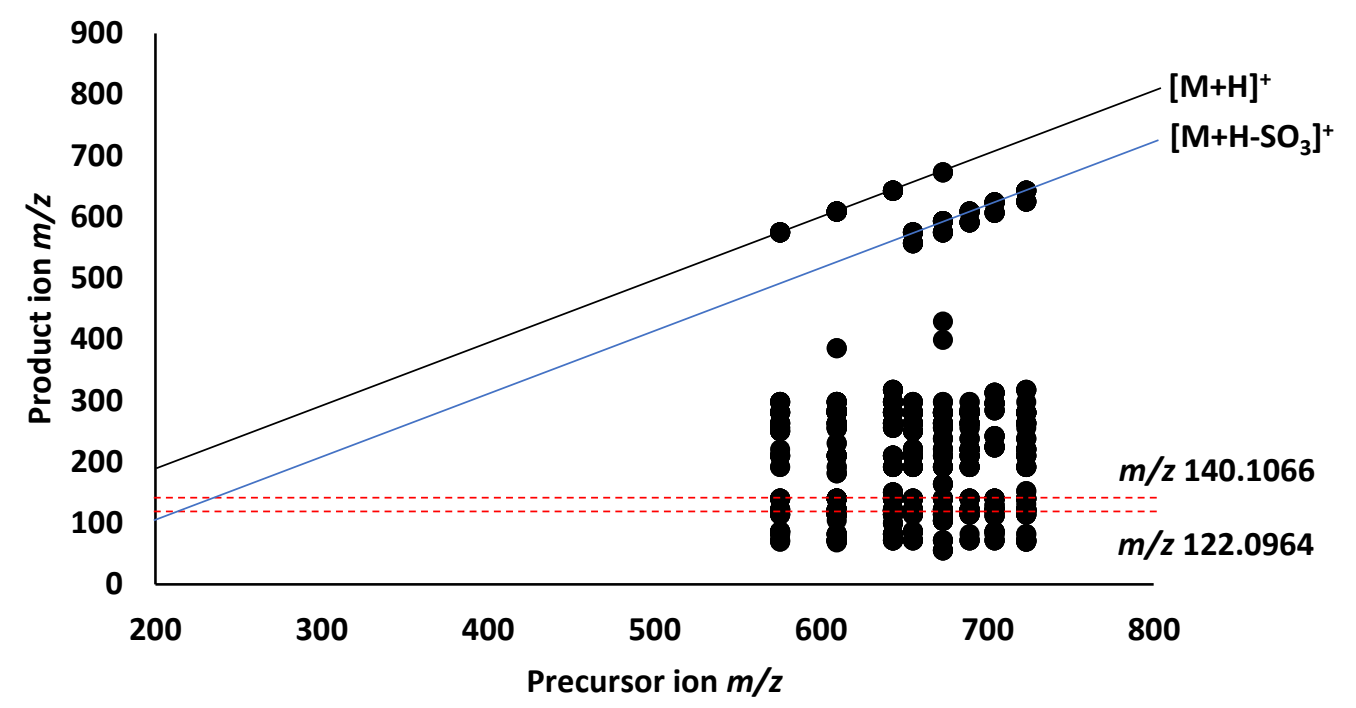

Figure 3.22: DFF plot showing Choi containing cyanopeptides produced by $M$. aeruginosa CPCC 632 grown in BBM medium with the product ions $\mathrm{m} / \mathrm{z} 140.1066$ and $\mathrm{m} / \mathrm{z} 122.0964$.

The DFF of M. aeruginosa CPCC 632 shows a search for the pair of diagnostic product ions at $\mathrm{m} / \mathrm{z} 140.1066$ and 122.0964 and depicts eight potential aeruginosin metabolites with no metabolite matching literature reports or cyanopeptide databases (Table 3.8). Five of these metabolites display a product ion with a neutral loss of 79.9561 suggesting the loss of a sulfate group, which is depicted by the blue diagonal line (Figure 3.22). The data points directly below the point for the neutral loss 
of the sulfate, is the neutral loss of water or dehydration $\left[\mathrm{M}+\mathrm{H}-\mathrm{H}_{2} \mathrm{O}-\mathrm{SO}_{3}\right]^{+}$. The aeruginosins produced by M. aeruginosa CPCC 632 are further classified as containing a Tyr or a Phe at residue 2 based on the presence of the immonium ions for Tyr $(m / z$ 136.0756) or Phe $(m / z 120.0807)$ within their MS/MS spectra (Figure 3.21). Chlorination of the Hpla amino acid within aeruginosin metabolites was confirmed via the mass defect of chlorine, visible within the high-resolution mass of the precursor ion as well as by isotopic abundance of the $\mathrm{M}+2$ peaks.

A search for the diagnostic product ions generated for the Choi moiety within the MS/MS data for a M. aeruginosa CPCC 633 extract resulted in seven aeruginosin-like metabolites with no previous reports of these compounds within the literature (Table 3.8). This strain shares the production of aeruginosin 688 and aeruginosin 608 with $M$. aeruginosa CPCC 632. The aeruginosin like compounds produced by M. aeruginosa CPCC 633 seem to have substitutions of Phe or Lxx within position 2. Interpretation of the MS/MS data for these compounds revealed the characteristic Choi product ions and one of the following immonium ions: Phe $(\mathrm{m} / \mathrm{z} 120.0807)$ or $\mathrm{Lxx}(\mathrm{m} / \mathrm{z}$ 86.0969). Similar to M. aeruginosa CPCC 632, chlorination of the Hpla amino acid was deemed possible with no clear pattern.
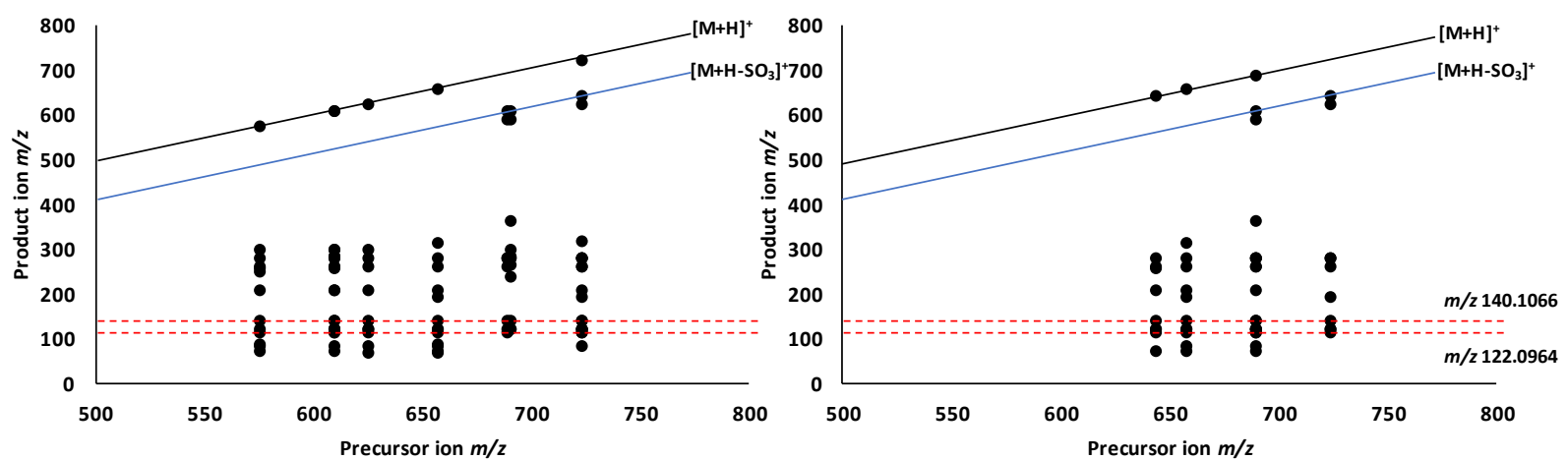

Figure 3.23: Diagnostic fragmentation filter plot showing Choi containing metabolites found in bloom samples 5 (left) and 8 (right), with the product ion $m / z 140.1066\left([\mathrm{Choi}+\mathrm{H}]^{+}\right)$and 122.0964 $\left(\left[\mathrm{Choi}-\mathrm{H}_{2} \mathrm{O}+\mathrm{H}\right]^{+}\right)$. 
This semi-targeted approach for the detection of Choi-containing molecules is effective within surface water samples (Figure 3.23). Six potential aeruginosins were detected using a search for the pair of diagnostic product ions specific to the Choi functionality (Table 3.7). A similar pattern of $[\mathrm{M}+\mathrm{H}]^{+}$and $\left[\mathrm{M}+\mathrm{H}-\mathrm{SO}_{3}\right]^{+}$was observed. Further, some metabolites precursor ions have isotopic patterns suggesting chlorination. This alongside the presence of the two characteristic product ions of the Choi moiety, suggests the compounds found within this environmental samples are aeruginosins. Majority of the samples, cultured Microcystis strains included, share the two potential aeruginosins, As $688\left(\mathrm{~m} / \mathrm{z} 689.2958[\mathrm{M}+\mathrm{H}]^{+}\right)$and As $722\left(\mathrm{~m} / \mathrm{z} 723.2574[\mathrm{M}+\mathrm{H}]^{+}\right)$. The profiles of aeruginosins are not consistent between the six environmental samples screened (Figure 3.23, Table 3.8). These differences suggest multiple cyanobacterial colonies resided within the water where the samples were collected.

Table 3.8: Chemical information of the Choi-containing metabolites produced by $M$. aeruginosa CPCC 300 and CPCC 632 and found within environmental water and bloom samples detected by DFF.

\begin{tabular}{|c|c|c|c|c|c|c|c|c|c|c|c|c|c|c|}
\hline \multirow[b]{2}{*}{ Name } & \multirow[b]{2}{*}{$\begin{array}{c}\mathbf{M}+\mathbf{H}]^{+} \\
m / z\end{array}$} & \multirow[b]{2}{*}{$\begin{array}{c}\text { Molecular } \\
\text { formula }\end{array}$} & \multirow[b]{2}{*}{$\begin{array}{l}\text { Error } \\
(\mathrm{ppm})\end{array}$} & \multirow[b]{2}{*}{$\begin{array}{c}\text { RT } \\
(\mathrm{min})\end{array}$} & \multirow[b]{2}{*}{$\begin{array}{c}\text { Residue } \\
1 \\
\end{array}$} & \multirow[b]{2}{*}{$\begin{array}{c}\text { Residue } \\
2\end{array}$} & \multicolumn{2}{|c|}{$\begin{array}{l}\text { M. aeruginosa } \\
\text { strains }\end{array}$} & \multicolumn{6}{|c|}{ Environmental sample \# } \\
\hline & & & & & & & $\begin{array}{c}\text { CPCC } \\
300\end{array}$ & $\begin{array}{c}\text { CPCC } \\
632 \\
\end{array}$ & 1 & 2 & 4 & 5 & 8 & 9 \\
\hline As 608 & 609.3397 & $\mathrm{C}_{32} \mathrm{H}_{44} \mathrm{~N}_{6} \mathrm{O}_{6}$ & -1.68 & 2.92 & Hpla & Phe & + & + & - & - & + & + & - & + \\
\hline As 650 & 651.3139 & $\mathrm{C}_{33} \mathrm{H}_{42} \mathrm{~N}_{6} \mathrm{O}_{6}$ & 0.70 & 2.40 & Hpla & Tyr & + & - & - & - & - & + & - & - \\
\hline As 684 & 685.2758 & $\mathrm{C}_{33} \mathrm{H}_{41} \mathrm{~N}_{6} \mathrm{O}_{6} \mathrm{Cl}$ & 0.63 & 2.32 & Cl-Hpla & Tyr & + & - & - & - & - & - & - & - \\
\hline As 688 & 689.2958 & $\mathrm{C}_{32} \mathrm{H}_{44} \mathrm{~N}_{6} \mathrm{O}_{9} \mathrm{~S}$ & -1.34 & 2.45 & Cl-Hpla & Phe & + & + & + & - & + & + & + & + \\
\hline As 724 & 725.2709 & $\mathrm{C}_{35} \mathrm{H}_{41} \mathrm{~N}_{6} \mathrm{O}_{9} \mathrm{Cl}$ & 1.31 & 2.55 & Cl-Hpla & Tyr & + & - & - & + & - & - & - & - \\
\hline As 574 & 575.3543 & $\mathrm{C}_{29} \mathrm{H}_{46} \mathrm{~N}_{6} \mathrm{O} 3$ & -0.61 & 2.41 & Hpla & Lxx & - & + & - & - & - & + & - & - \\
\hline As 642 & 643.2984 & $\mathrm{C}_{32} \mathrm{H}_{43} \mathrm{~N}_{6} \mathrm{O}_{6} \mathrm{Cl}$ & -1.38 & 2.71 & Cl-Hpla & Phe & - & + & - & - & - & - & - & - \\
\hline As 654 & 655.3111 & $\mathrm{C}_{29} \mathrm{H}_{46} \mathrm{~N}_{6} \mathrm{O}_{9} \mathrm{~S}$ & 0.22 & 2.39 & Hpla & Lxx & - & + & - & - & - & - & - & - \\
\hline As 703 & 704.3313 & $\mathrm{C}_{40} \mathrm{H}_{43} \mathrm{~N}_{6} \mathrm{O}_{6}$ & -0.02 & 2.31 & Hpla & Phe & - & + & - & - & - & - & - & - \\
\hline As 722 & 723.2574 & $\mathrm{C}_{32} \mathrm{H}_{43} \mathrm{~N}_{6} \mathrm{O}_{9} \mathrm{SCl}$ & -1.73 & 2.58 & Cl-Hpla & Phe & - & + & + & - & + & + & + & + \\
\hline As 602 & 603.3499 & $\mathrm{C}_{30} \mathrm{H}_{46} \mathrm{~N}_{6} \mathrm{O}_{7}$ & 0.38 & 2.31 & Hpla & Lxx & - & - & + & - & - & - & - & - \\
\hline As 634 & 635.3775 & $\mathrm{C}_{31} \mathrm{H}_{50} \mathrm{~N}_{6} \mathrm{O}_{8}$ & -0.43 & 2.30 & Hpla & Lxx & - & - & + & - & - & - & - & - \\
\hline As 652 & 653.3052 & $\mathrm{C}_{30} \mathrm{H}_{45} \mathrm{~N}_{6} \mathrm{O}_{8} \mathrm{Cl}$ & -0.84 & 2.47 & Cl-Hpla & Lxx & - & - & - & + & - & - & - & - \\
\hline As 624 & 625.3343 & $\mathrm{C}_{32} \mathrm{H}_{44} \mathrm{~N}_{6} \mathrm{O}_{7}$ & 0.05 & 2.40 & Cl-Hpla & Phe & - & - & - & - & - & + & - & - \\
\hline As 656 & 657.3256 & $\mathrm{C}_{32} \mathrm{H}_{44} \mathrm{~N}_{6} \mathrm{O}_{9}$ & -0.64 & 2.34 & Hpla & Lxx & - & - & - & - & - & + & + & - \\
\hline As 642* & 643.3016 & $\mathrm{C}_{32} \mathrm{H}_{44} \mathrm{~N}_{6} \mathrm{O}_{6} \mathrm{Cl}$ & 0.18 & 2.54 & Cl-Hpla & Phe & - & - & - & - & + & - & + & - \\
\hline
\end{tabular}




\subsubsection{Semi-targeted analysis: Microginins}

The structural variability of microginin congeners arises not only with the amino acid sequence but with the chain length, methylation, and chlorination of the N-terminal PKS aliphatic chain (Strangman \& Wright, 2016; Welker et al., 2006). The fragmentation of the N-terminus and thus the aliphatic portion of these molecules results in diagnostic product ions that are distinguishable from the immonium ions of proteinaceous amino acids (Table 3.9, Figure 3.24). The formula for the diagnostic product ions is confirmed with inspection of ${ }^{15} \mathrm{~N}$-labelled experiments (Figure 3.24). The N-terminal moiety may be an octanoic acid derivative (Ahoa) or a decanoic acid derivative, which is added on with a PKS synthase. Methylation of the N-terminal polyketide chain on the amino moiety can occur and results in an entirely different set of microginin metabolites. This chain may be halogenated on the terminal carbon, resulting in non-, mono-, di-, and tri-halogenated congeners which can be deduced by investigation of the isotope patterns of the parent ions within the MS/MS (Figure 3.7). Multiple chlorination states were visible within the MS/MS as a mass difference of 1 chlorine atom $(\Delta 33.9606)$ when comparing product ions within the non-chlorinated form and the mono-chlorinated form. 

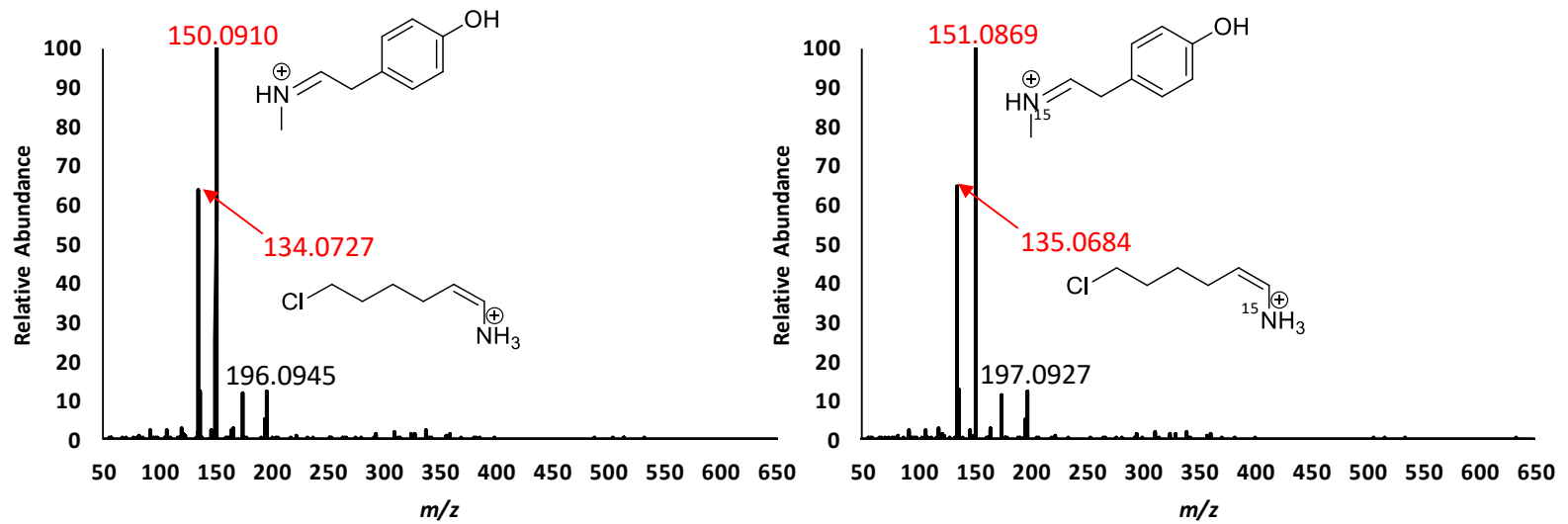

Figure 3.24: MS/MS spectra for microginin 646 are displayed when cyanobacteria were grown in BG-11 (left) and $\mathrm{Na}^{15} \mathrm{NO}_{3}$ supplemented BG-11 (right) media. (Top left) Diagnostic product ions for microginin 646 at $\mathrm{m} / \mathrm{z} 150.0910$ and $\mathrm{m} / \mathrm{z} 134.0727$ correspond to the NMeTyr and Cl-Ahoa, respectively. Incorporation of ${ }^{15} \mathrm{~N}$ into peptide scaffolds supports these diagnostic product ions. Corresponding NMeTyr and Cl-Ahoa product ions are observed at $\mathrm{m} / \mathrm{z} 151.0869$ and $\mathrm{m} / \mathrm{z} 135.0684$ that contain 1 nitrogen atom each.

Table 3.9: Product ions specific to the variable N-terminal polyketide moiety present within metabolites of the microginin chemical class.

\begin{tabular}{|c|c|c|c|}
\hline \multirow{2}{*}{ Amino acid sequence } & \multicolumn{3}{|c|}{ Product ion $\boldsymbol{m} / \boldsymbol{z}$} \\
\cline { 2 - 4 } & Non-chlorinated & Mono-chlorinated & Di-chlorinated \\
\hline Ahoa & 100.1122 & 134.0727 & 168.0334 \\
\hline NMe-Ahoa & 114.1278 & 148.0883 & 182.0483 \\
\hline Ahda & 128.1433 & 162.1039 & 196.0639 \\
\hline NMe-Ahda & 142.1589 & 176.1195 & 210.0795 \\
\hline
\end{tabular}

To conduct a search for microginins within Microcystis extracts, one product ion characteristic of the N-terminal polyketide moiety is used as a pair of diagnostic product ions could not be established. Using all possible N-terminal polyketide moiety product ions described in Table 3.9, a search for all microginins was performed and compiled into one plot (Figure 3.25). This encapsulated the four different chain types (Ahoa, NMeAhoa, Ahda, NMeAhda) and the three different chlorination states (non-, mono-, di-). 2 M. aeruginosa strains CPCC 632 and CPCC 633 produce different mixtures of microginins, with the two previously reported congeners microginin 
646 and microginin 680 produced by both strains and the congener microginin 613 exclusively produced by M. aeruginosa CPCC 633 (Table 3.10, Strangman \& Wright, 2016).

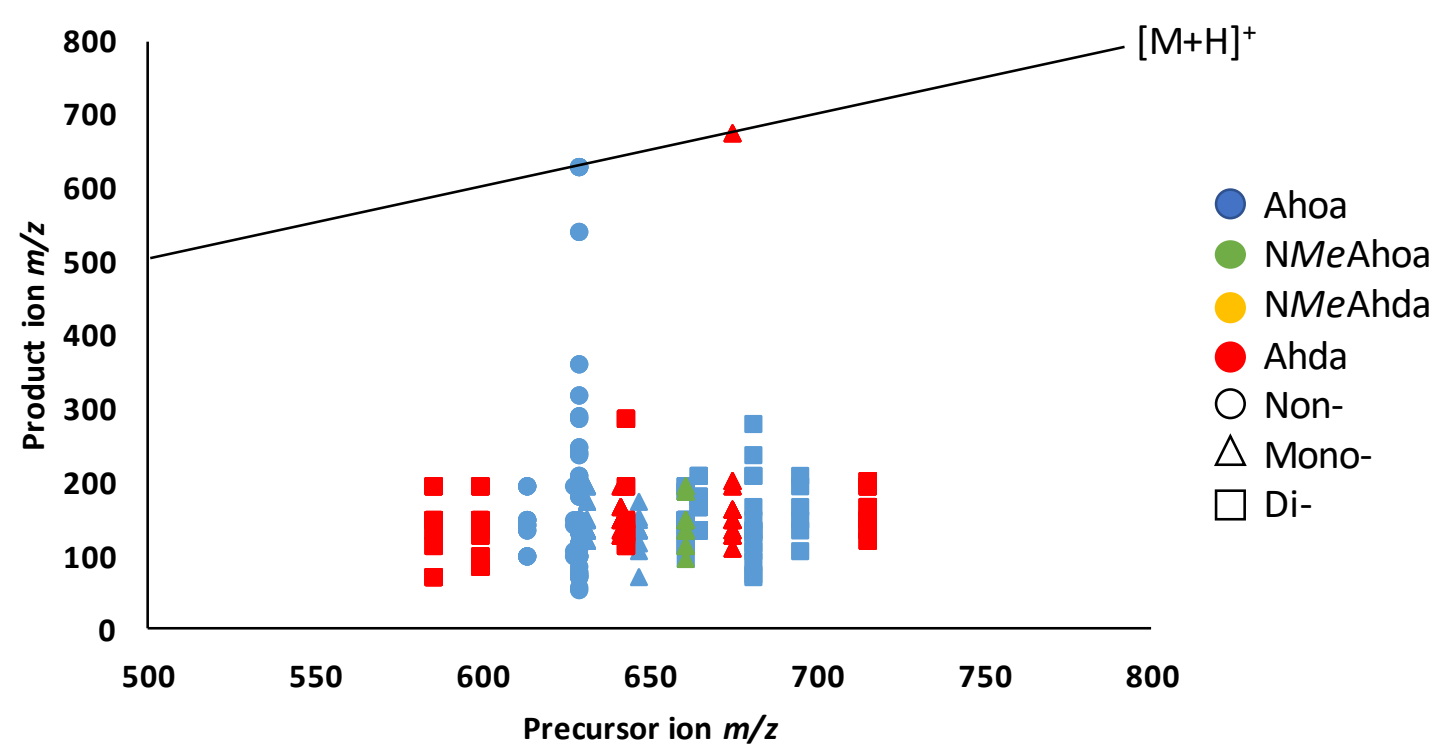

Figure 3.25: Diagnostic fragmentation filter plot showing microginins produced by M. aeruginosa CPCC 633 when searching for the different product ions that correlate to the $\mathrm{N}$-terminal polyketide moiety. Colours are used to display the variable chain length (Ahoa, NMeAhoa, Ahda, NMeAhda) and shape to designate the chlorination state (non-, mono-, di-).

M. aeruginosa CPCC 632 produced eight microginin congeners, two are non-chlorinated, four are chlorinated, and two are di-chlorinated (Table 3.10). All contain the Ahoa N-terminal chain with two congeners methylated on the amino group. M. aeruginosa CPCC 633 produces fifteen microginin congeners, four are non-chlorinated, four are mono-chlorinated, and seven are dichlorinated (Table 3.10). This strain produces ten congeners with the Ahoa N-terminal polyketide moiety with one being methylated on the amino group and it produces five congeners with the Ahda N-terminal polyketide moiety.

This screening technique for microginin congeners was performed on MS/MS data sets generated from environmental samples collected in the National Capital Region (Figure 3.25, Table 3.10). 

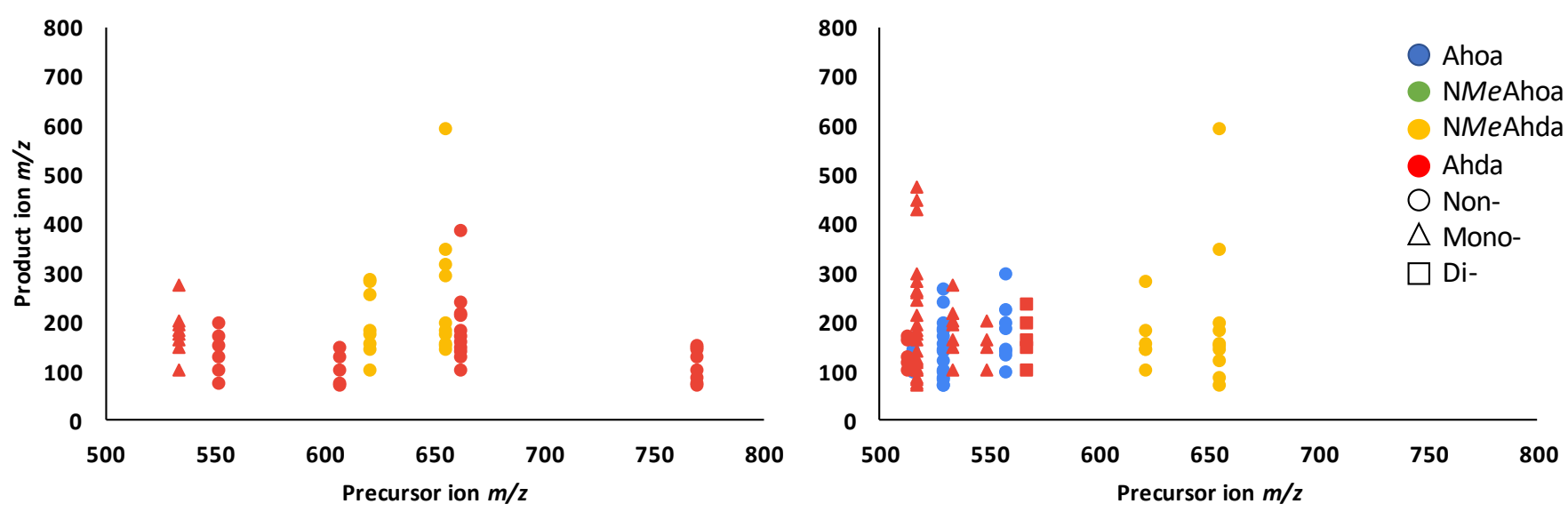

Figure 3.25: Representative diagnostic fragmentation filter plots of microginins detected within environmental samples 4 (left) and 8 (right) when searching for the different product ions that correlate to the N-terminal polyketide moiety. Colours are used to display variable chain length and substitution (Ahoa, NMeAhoa, Ahda, NMeAhda) and shape to designate the chlorination state (non-, mono-, di-).

The microginin profile for the six investigated surface water and bloom samples were diverse suggesting multiple cyanobacterial populations growing within the sampled area. 28 microginin congeners were detected with no overlap in the metabolites identified by the two M. aeruginosa strains studied (CPCC 632 and CPCC 633). Of the twenty-eight microginins identified within the environmental samples, six have been previously reported, microginin KR 604, microginin 551, microginin 607B, microginin 621B, microginin FR12, and microginin 478 (Stewart et al., 2018). Thirteen of the detected congeners contain an Ahda N-terminal polyketide moiety, nine contain an NMeAhda moiety, and six contain the Ahoa N-terminal polyketide moiety. Five of these detected microginins were mono-chlorinated and only one congener was di-chlorinated. 
Table 3.10: Chemical information of the microginins produced by M. aeruginosa CPCC 632 and CPCC 633 and found in surface water and bloom samples.

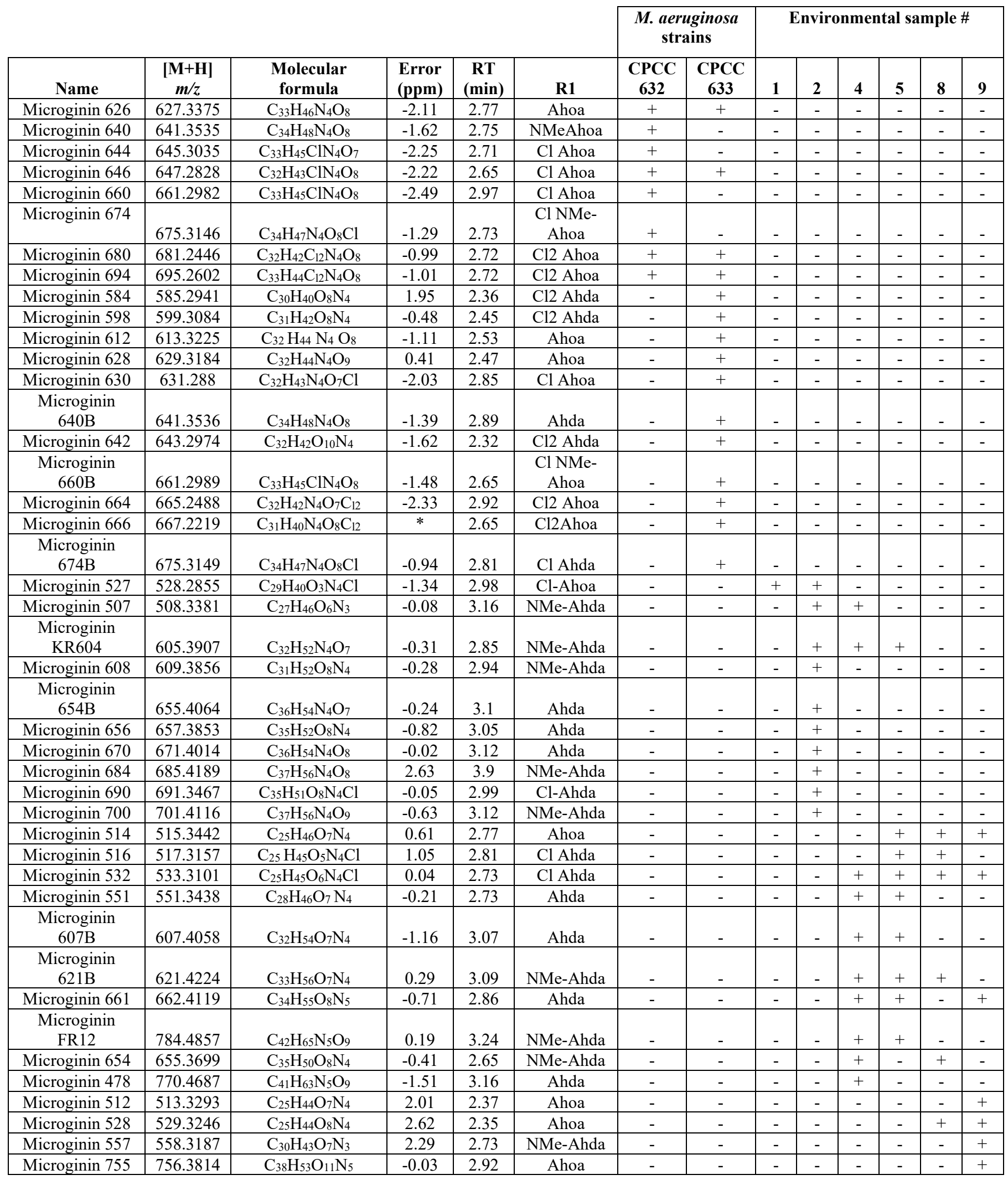




\begin{tabular}{|c|c|c|c|c|c|c|c|c|c|c|c|c|c|}
\hline $\begin{array}{c}\text { Microginin } \\
\text { 512B }\end{array}$ & 513.3644 & $\mathrm{C}_{26} \mathrm{H}_{49} \mathrm{O}_{6} \mathrm{~N}_{4}$ & -0.54 & 2.95 & Ahda & - & - & - & - & - & - & + & - \\
\hline Microginin 548 & 549.3046 & $\mathrm{C}_{25} \mathrm{H}_{45} \mathrm{O}_{7} \mathrm{~N}_{4} \mathrm{Cl}$ & -0.69 & 2.69 & $\mathrm{Cl}$ Ahda & - & - & - & - & - & - & + & - \\
\hline Microginin 556 & 557.355 & $\mathrm{C}_{27} \mathrm{H}_{48} \mathrm{O}_{8} \mathrm{~N}_{4}$ & 0.87 & 2.98 & Ahoa & - & - & - & - & - & - & + & - \\
\hline Microginin 566 & 567.2712 & $\mathrm{C}_{25} \mathrm{H}_{44} \mathrm{O}_{6} \mathrm{~N}_{4} \mathrm{C}_{12}$ & 0.19 & 2.97 & $\mathrm{Cl} 2$ Ahda & - & - & - & - & - & - & + & - \\
\hline
\end{tabular}

\section{$\underline{3.3 \text { Cyanobacterial growth assessment }}$}

Biological indicators (cell count, $\mathrm{OD}_{750}, \mathrm{pH}$, dried biomass) were monitored to investigate growth and growth rates of four Microcystis strains studied in the three culture media BG-11, BBM, and MA. Growth rates were calculated using cell counts after 25 days in all experiments for normalization (Table 3.11). Growth rates remained relatively consistent between BG-11 and BBM culture media for the M. aeruginosa strains CPCC 299, CPCC 300, CPCC 632, and CPCC 633, yet increased significantly in BBM for the M. flos-aquae strain CPCC 461. In all cases, the growth rate was lowest when grown in MA culture medium (Table 3.11). The major components of BBM and BG-11 media are similar with the largest differences being the concentrations of the essential nutrients, nitrogen and phosphorous. BBM media has a lower concentration of the essential nutrient $\mathrm{NaNO}_{3}\left(0.75 \mathrm{~g} \mathrm{~L}^{-1}\right)$ and two sources of phosphorous $\left(\mathrm{KH}_{2} \mathrm{PO}_{4}\right.$ and $\left.\mathrm{K}_{2} \mathrm{HPO}_{4}\right)$, whereas BG11 has double the concentration of nitrogen $\left(1.5 \mathrm{~g} \mathrm{~L}^{-1}\right)$ and one source of phosphorous $\left(\mathrm{K}_{2} \mathrm{HPO}_{4}\right)$. Another nutrient difference between these two culture media is the iron source which has been shown to impact toxin production and growth rate, $\mathrm{BBM}$ has $\mathrm{FeSO}_{4} \bullet 7 \mathrm{H}_{2} \mathrm{O}$ and $\mathrm{BG}-11$ has ferric ammonium citrate. MA media has many nutrient differences with BG-11 and BBM, starting with the major nitrogen source being $\mathrm{KNO}_{3}$ and the major phosphorous source being $\beta-\mathrm{Na}_{2}$ glycerophosphate. The source of iron also differs, being $\mathrm{FeCl}_{3} \bullet 6 \mathrm{H}_{2} \mathrm{O}$.

As two of the investigated strains are known MC producers, the phosphatase activity of all Microcystis extracts was investigated with a PP2A inhibition assay (Table 3.11). There was no activity from the extracts from M. aeruginosa CPCC 632 and CPCC 633. As expected, activity 
was seen by the toxic strains M. aeruginosa CPCC 299 and CPCC 300, with M. aeruginosa CPCC 299 showing a greater activity than M. aeruginosa CPCC 300. Unexpectedly, M. flos-aquae CPCC 461 showed high inhibition of the phosphatase enzyme with the highest concentration of "MC equivalents" although this strain does not produce MCs nor anabaenapeptins. Investigation into the toxins produced by this M. flos-aquae CPCC 461 strain would provide bioactive metabolites against PP2A.

Table 3.11: Strain information and specific growth rates $\left(\mathrm{day}^{-1}, \mu\right)$ at $27^{\circ} \mathrm{C}$ when grown in BBM, BG-11 and MA.

\begin{tabular}{|c|c|c|c|c|c|c|}
\hline \multirow{2}{*}{ Strain } & \multicolumn{2}{|c|}{ Collection data } & \multicolumn{2}{|c|}{ Specific growth rate, day $^{-1}(\boldsymbol{\mu})$} & \multicolumn{1}{c|}{$\begin{array}{c}\text { PP2A inhibition } \\
\text { ([MC] equivalent } \boldsymbol{\mu} \mathbf{~ L ~}^{-1} \text { ) }\end{array}$} \\
\cline { 2 - 5 } $\begin{array}{c}\text { Date } \\
\begin{array}{c}\text { M. aeruginosa } \\
\text { CPCC 299 }\end{array}\end{array}$ & $08 / 90$ & $\begin{array}{c}\text { Pretzlaff Pond } \\
\text { AB, Canada }\end{array}$ & 0.240 & 0.255 & 0.195 & 0.71 \\
\hline $\begin{array}{c}\text { M. aeruginosa } \\
\text { CPCC 300 }\end{array}$ & $08 / 90$ & $\begin{array}{c}\text { Pretzlaff Pond } \\
\text { AB, Canada }\end{array}$ & 0.235 & 0.227 & 0.316 & 0.29 \\
\hline $\begin{array}{c}\text { M. flos-aquae } \\
\text { CPCC 461 }\end{array}$ & $08 / 75$ & $\begin{array}{c}\text { Fox River } \\
\text { WI, USA }\end{array}$ & 0.325 & 0.309 & 0.103 & 0.82 \\
\hline $\begin{array}{c}\text { M. aeruginosa } \\
\text { CPCC 632 }\end{array}$ & $10 / 48$ & $\begin{array}{c}\text { Lake Mendota } \\
\text { WI, USA }\end{array}$ & 0.250 & 0.345 & 0.139 & ND \\
\hline $\begin{array}{c}\text { M. aeruginosa } \\
\text { CPCC 633 }\end{array}$ & $10 / 54$ & $\begin{array}{c}\text { Little Rideau } \\
\text { Lake, ON } \\
\text { Canada }\end{array}$ & 0.260 & 0.291 & 0.181 & ND \\
\hline
\end{tabular}

A media composition comparison was performed with four of the five Microcystis strains to understand the effects media has on cyanobacterial growth and oligopeptide cyanopeptide production. The two media used in this experiment were BG-11 and MA as they showed major differences in growth rates in preliminary experiments which greatly impact toxin production.

Each strain was inoculated to a cell number on day 0 (high $10^{4}$-low $10^{5}$ cells $\mathrm{mL}^{-1}$ ) and biological growth indicators such as cell $\mathrm{mL}^{-1}$ and dried biomass, were monitored for triplicate cultures every 5 days until the cells reached mid $10^{7}$ cells $\mathrm{mL}^{-1}$ (Figure 3.26). 

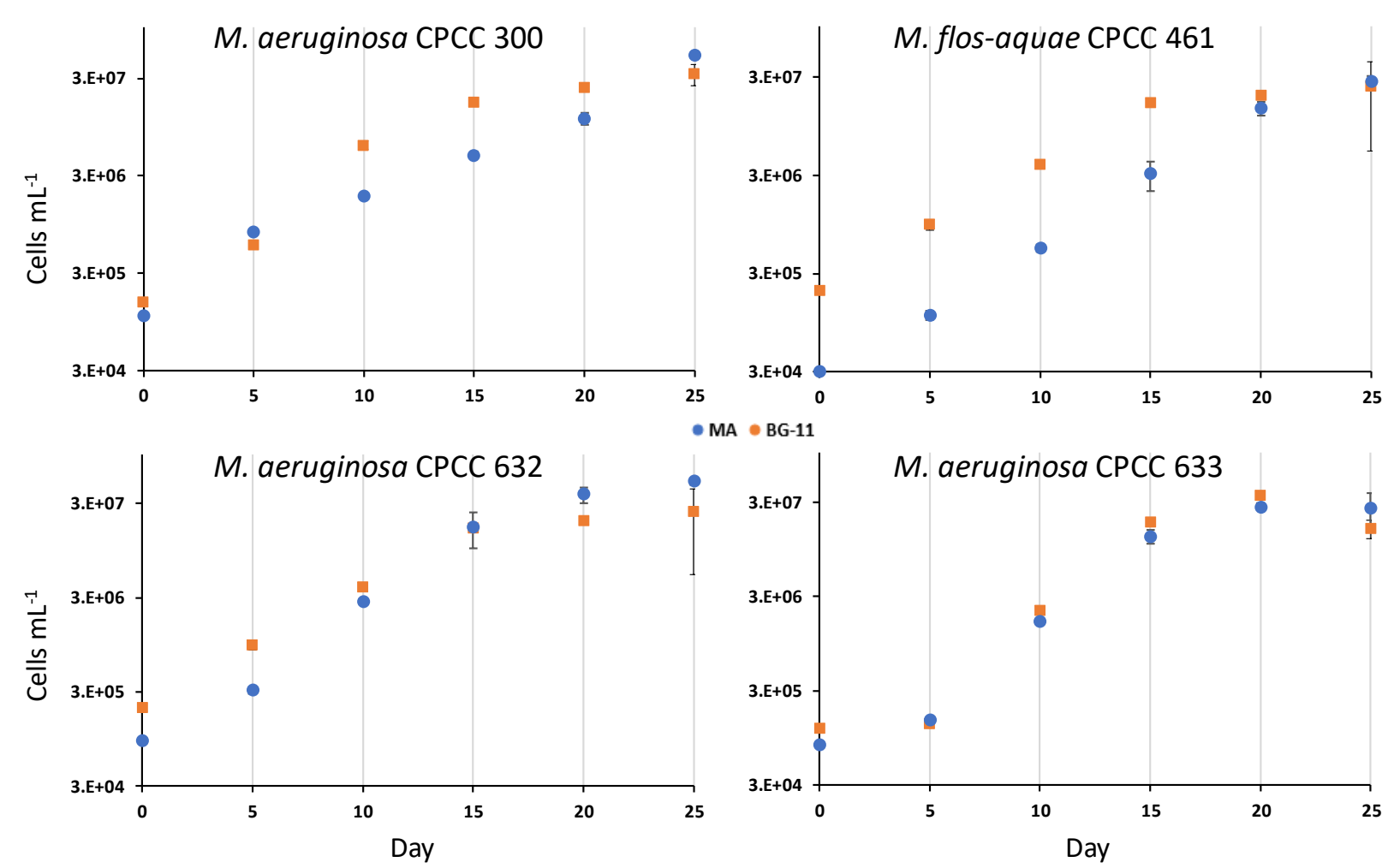

Figure 3.26: Growth data for M. aeruginosa CPCC 300 (top left), M. flos-aquae CPCC 461 (top right), M. aeruginosa CPCC 632 (bottom left), and M. aeruginosa CPCC 633 (bottom right) showing cells $\mathrm{mL}^{-1}$ measurements at each time interval. Orange data points indicate measurements when strains were grown in MA media and blue data points indicate measurements when strains were grown in BG-11 media.

Cell number increased for all Microcystis strains studied over the course of 25 days (Figure 3.26).

In most cases, cell numbers reached a maximum at day 20 and began to plateau as the cells entered stationary phase. This is well represented with the growth of CPCC 633 (bottom right) as the peak cell number is day 20 in both media and either decreases or stays the same in the final reading on day 25 (Figure 3.26). All strains entered the stationary phase within the range of $3.12 \times 10^{7}-$ $5.24 \times 10^{7}$ cells $\mathrm{mL}^{-1}$ with this end of exponential growth variable based on the strain and the culture media. 

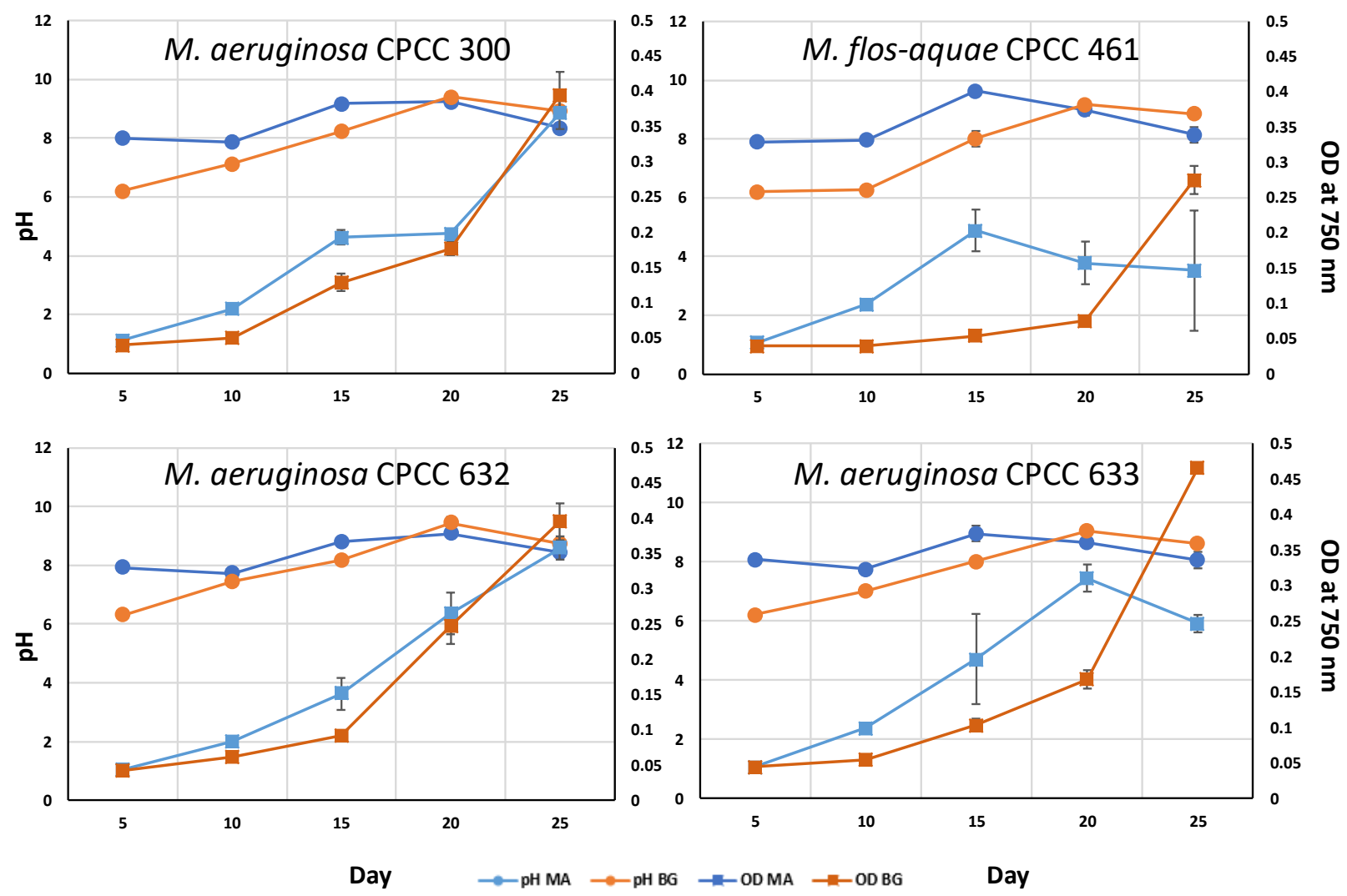

Figure 3.27: $\mathrm{pH}$ and optical density at $750 \mathrm{~nm}$ for M. aeruginosa CPCC 300 (top left), M. flosaquae CPCC 461 (top right), M. aeruginosa CPCC 632 (bottom left), and $M$. aeruginosa CPCC 633 (bottom right) grown in BG-11 (orange) and MA (blue) growth media. $\mathrm{pH}$ data points are represented by circles and optical density data points are represented with squares.

The $\mathrm{pH}$ of the culture media BG-11 and MA were adjusted prior to inoculation to 7.5 and 8.6, respectively. There is an increase in the extracellular $\mathrm{pH}$ over the 25-day time course for all Microcystis cultures (Figure 3.27). The maximum $\mathrm{pH}$ for each culture occurred at the end of the exponential phase of growth, with a decrease as the cells progressed in the stationary phase. In all cases, the $\mathrm{pH}$ at day 25 was greater when the strains were grown in BG-11 media versus MA. Chlorophyll-a absorbance at $750 \mathrm{~nm}$ was utilized as a proxy of bacterial growth. Optical density of the liquid cultures increased in all cases as the cells replicated exponentially. 


\subsection{Investigation of cyanopeptide production by Microcystis strains}

With the untargeted MS metabolomics approach, we can view the entire chemical profile of a strain and compare in depth any changes that may occur due to varying growth conditions. The untargeted data presented within the PCA, loading plot, and molecular networking analysis provided identification of cyanopeptides specific to the different Microcystis strains. An understanding of their unique metabolomes allows for investigation of compounds changes when growth conditions are varied. With intracellular extracts at time points along the established growth curve, the production of each cyanopeptide is monitored with normalized peak area to compare relative cyanopeptide amounts. For the MC producer, M. aeruginosa CPCC 300, intracellular $\mathrm{MC}$ concentrations can be quantified with reference material. All values are normalized with dried biomass (mg). Results from these studies will help determine the optimal conditions for the production of understudied cyanopeptide groups including cyanopeptolins and microginins.

\subsubsection{Microcystin quantification in toxigenic strains}

The identification and quantification of MC congeners within the toxic Microcystis extracts was simplified with the use of reference materials. Chemical information such as retention time, precursor ion $\mathrm{m} / \mathrm{z}$, product ion $\mathrm{m} / \mathrm{z}$ and fragmentation patterns, as well as pseudomolecular ions were acquired for nine congeners (MC-LR, $\left[\mathrm{Asp}^{3}\right]$-MC-LR, MC-RR, MC-LY, MC-LA, MC-WR, MC-YR, MC-LF, NOD) and is summarized in Table 3.2. A standard curve with a concentration range of a mixture of MC standards, quantification of $\mathrm{MC}$ congeners is possible. 
Table 3.12: Summary of LC-HRMS data for $8 \mathrm{MC}$ congeners and NOD with reference material.

\begin{tabular}{|l|l|l|l|l|}
\hline Name & $\boldsymbol{m} / \boldsymbol{z}[\mathbf{M}+\mathbf{H}]^{+}$ & RT (min) & Formula & Delta (ppm) \\
\hline MC-LR & 995.5565 & 3.34 & $\mathrm{C}_{49} \mathrm{H}_{74} \mathrm{~N}_{10} \mathrm{O}_{12}$ & 1.76 \\
\hline$\left[\right.$ Asp $\left.{ }^{3}\right]-M C-L R$ & 981.5385 & 3.34 & $\mathrm{C}_{48} \mathrm{H}_{72} \mathrm{~N}_{10} \mathrm{O}_{12}$ & -0.71 \\
\hline MC-RR & 1038.5726 & 2.78 & $\mathrm{C}_{49} \mathrm{H}_{75} \mathrm{~N}_{13} \mathrm{O}_{12}$ & -0.47 \\
\hline MC-LY & 1002.5176 & 5.29 & $\mathrm{C}_{52} \mathrm{H}_{71} \mathrm{~N}_{7} \mathrm{O}_{13}$ & -0.66 \\
\hline MC-LA & 910.4913 & 5.15 & $\mathrm{C}_{46} \mathrm{H}_{67} \mathrm{~N}_{7} \mathrm{O}_{12}$ & -0.82 \\
\hline MC-WR & 1068.5509 & 3.61 & $\mathrm{C}_{54} \mathrm{H}_{73} \mathrm{~N}_{11} \mathrm{O}_{12}$ & -0.36 \\
\hline MC-YR & 1045.5349 & 3.26 & $\mathrm{C}_{52} \mathrm{H}_{72} \mathrm{~N}_{10} \mathrm{O}_{13}$ & -0.39 \\
\hline MC-LF & 986.5229 & 5.78 & $\mathrm{C}_{52} \mathrm{H}_{71} \mathrm{~N}_{7} \mathrm{O}_{12}$ & -0.45 \\
\hline NOD & 825.4496 & 3.07 & $\mathrm{C}_{41} \mathrm{H}_{60} \mathrm{~N}_{8} \mathrm{O}_{10}$ & -1.11 \\
\hline
\end{tabular}

Production for the congener, MC-LR, was quantified with standard curves for the "toxic" strain M. aeruginosa CPCC 300 (Table 3.13, Supplementary info XX).

Table 3.13: Intracellular MC-LR levels for M. aeruginosa CPCC 300 grown in BBM media at $27^{\circ} \mathrm{C}$.

\begin{tabular}{|l|l|l|}
\hline Media & Maximum production & $\mu \mathrm{g}$ MC-LR $\mathrm{mg}^{-1}$ biomass \\
\hline BG-11 & Day 20 & 1.91 \\
\hline MA & Day 10 & 1.83 \\
\hline
\end{tabular}

The MC-LR production over time by M. aeruginosa CPCC 300 was monitored in triplicate culture for comparison of media effects on toxin production. M. aeruginosa CPCC 300 was grown in the two defined culture media, MA and BG-11, and the normalized amounts ( $\mu \mathrm{g} \mathrm{MC} \mathrm{mg}^{-1}$ biomass) produced over time are displayed in Figure 3.28. 


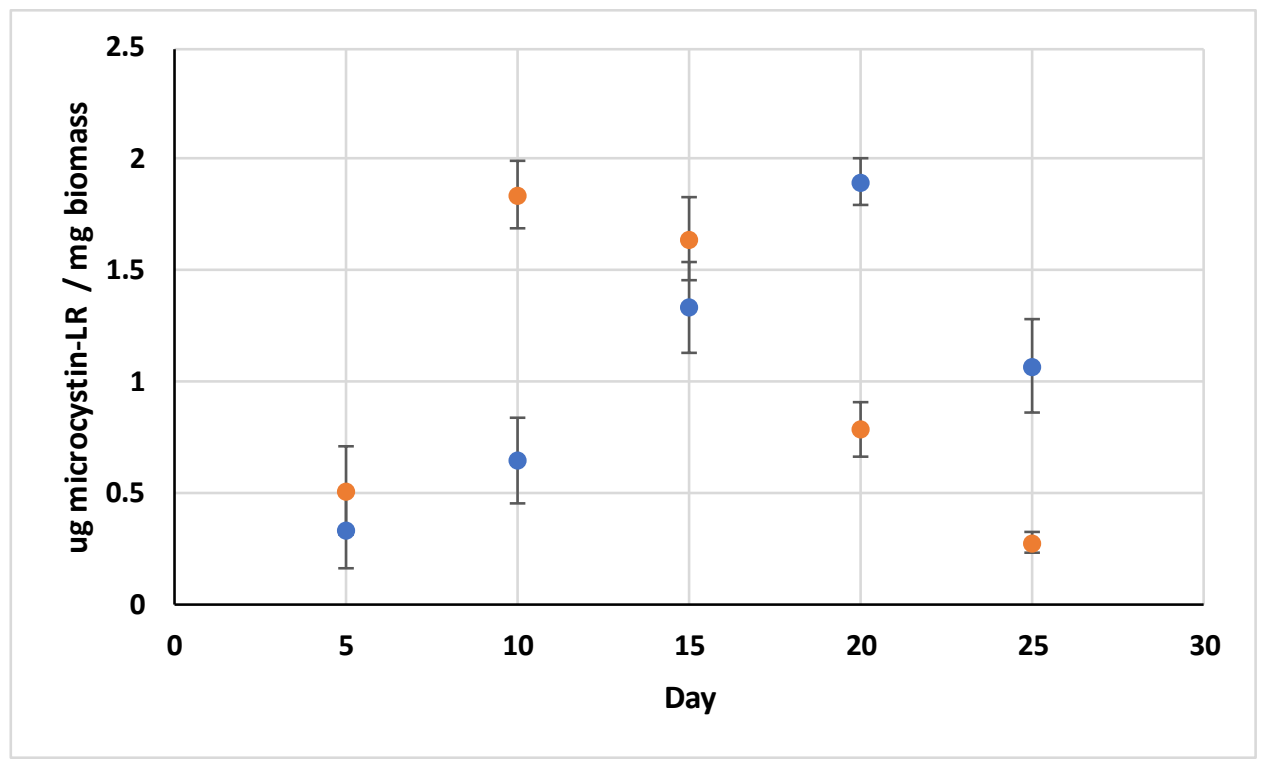

Figure 3.28: Quantification of intracellular MC-LR ( $\mu$ g MC-LR mg biomass ${ }^{-1}$ ) production by $M$. aeruginosa CPCC 300 when grown in the two culture media BG-11 (orange) and MA (blue).

M. aeruginosa CPCC 300 is known as a "toxic" Microcystis strain as it produces MC congeners. Quantification of the congener, MC-LR, was performed with a standard curve and production was variable between the two growth media, BG-11 and MA (Figure 3.28). The production of these metabolites is correlated with the growth rate and phase of growth. Intracellular MC-LR concentration spikes quickly at day 10 of growth when CPCC 300 was grown in MA and decreases steadily over the remaining time course. The growth curve for CPCC 300 grown in MA reaches the end of the exponential phase and the beginning of the stationary phase between day 10 and 15 , suggesting that the majority of toxin production occurs during the late exponential phase of growth. Max MC production is reported to occur by other strains in the late exponential, or early stationary phase (Watanabe et al., 1996). Our data for M. aeruginosa CPCC 300 appear to agree with previous findings. Looking at the BG-11 growth curve, the cells enter the stationary phase at day 20 of growth which correlates to the maximum intracellular concentration of MC-LR. 


\subsubsection{Effects of media composition on the production of cyanopeptides by Microcystis strains}

The metabolites presented within the molecular network were targeted for comparison of relative amounts (peak area/biomass) when the producing strain was grown in the two media, BG-11 and MA. Heat maps were generated for each strain and their respective metabolites in $\mathrm{R}$ software package with their average normalized peak areas using the packages: gplots and colorRamps. The overall production profile for all selected compounds can be visualized and compared against the other metabolites produced over time and between the two defined culture media. ANOVA pairwise comparison $(\mathrm{p}<0.005)$ and Tukey's LSD test (letters) were used to provide statistical evidence of toxin production over time as well as to compare the production levels of metabolites when grown in different culture media. Very few studies have examined the dynamics of non-MC cyanopeptides. These types of studies are complicated by the very few cyanopeptide reference materials available required for identification and quantitation.

\subsection{2a Media effects on cyanopeptide production by M. aeruginosa CPCC 300}

The "toxic" strain M. aeruginosa CPCC 300 produced multiple different chemical groups together with MCs such as cyanopeptolins, aeruginosins, and cyanobactins (Figure 3.3). Figure 3.29 demonstrates the relative amounts of specific metabolites and their production profile over 25 days when grown in two different culture media, BG-11 and MA. 

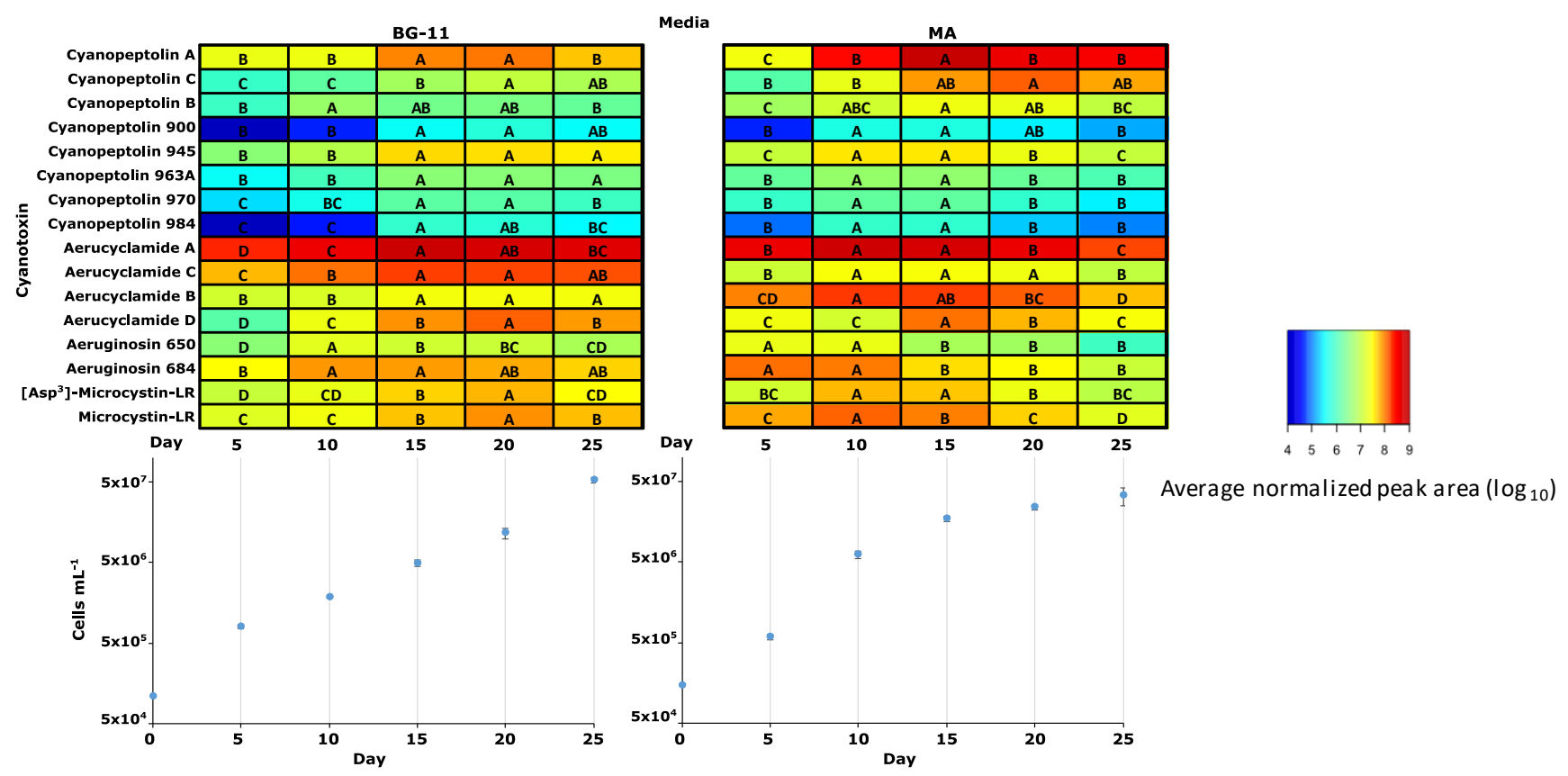

Figure 3.29: Heatmap showing cyanopeptide production by $M$. aeruginosa CPCC 300 over time in two growth media (BG-11; left and MA; right), generated with the average normalized peak area ( $\log _{10}$, top). Tukey's HSD test distinguished groups designated with letters to illustrate quantitative differences in the individual cyanopeptide production profile (statistically significant, $\mathrm{p}<0.05$ ). Data points with the same letter within a cyanopeptide row are not statistically significant from one another. Cell count measurements are depicted to correlate toxin production with the phase of growth (bottom).

The aerucyclamides produced by CPCC 300 appear to be present within the cells at higher amounts compared to other groups at all growth stages. While we could not quantify most cyanopeptides, this observation is in accordance with Natumi and Janssen et al. 2020. Aerucyclamide A and C have the darkest shade of red over the time course when grown in both media. The unknown cyanopeptolins appear to have the lowest concentrations of the represented compounds with blue to green shades over the time course. Of particular interest is the maximum intracellular concentrations, which occur at an earlier time point within the growth curve when the strain is grown in MA media whereas the maximum intracellular concentrations occur at a later time point within the growth curve when the strain is grown in BG-11 (Figure 3.29). A potential explanation for this is the accumulation of the toxin within the cell over time when the strain is grown in BG- 
11. The day 25 intracellular concentrations for all compounds when the strain is grown in MA appear to be the lowest suggesting that the metabolites are released from the cell upon lysis within the stationary phase and thus not accumulated intracellularly (Walls et al., 2018).

Table 3.14 compares the relative normalized peak area maximum for major metabolites produced by M. aeruginosa CPCC 300 when grown in two separate culture media, BG-11 and MA. Green cells indicate a larger peak area maximum and red indicates a smaller maximum. ANOVA was performed using triplicate peak areas extrapolated from $\mathrm{R}$ to assess media effects on intracellular concentrations of identified metabolites. Based on the growth curves generated by CPCC 300 when grown in the two culture media, MA appears to reach the maximum cell number per day at a faster rate than when the culture is grown in BG-11. This difference in growth rate suggests there may be differences in toxin quantities and peak production days. Based on the summary data in Table 3.14, majority of the maximum relative abundances occur on day 15 for both BG-11 and MA media although there are a larger number of day 20 maxima for BG-11 than MA which correlates to the differences in the growth curves. 7 of the 16 investigated compounds show a statistically significant difference in maximum relative abundance suggesting specific chemicals are produced in variable quantities when CPCC 300 is grown with different concentrations of essential nutrients and alternative nitrogen and phosphorous sources. Of the investigated chemical classes, cyanopeptolins are produced in significantly higher concentrations when CPCC 300 was grown in MA whereas cyanobactins and aeruginosins are produced in significantly higher concentrations when the strain is grown in BG- 11 . 
Table 3.14: Maximum relative abundance (green indicates greater peak area) for selected metabolites produced by CPCC 300 when grown in two culture media with an ANOVA pairwise comparison $(\mathrm{p}<0.05)$ to add significance to the media effects. The $\mathrm{p}$-values are bolded for statistically significant differences in relative peak area for the metabolites between the two growth media.

\begin{tabular}{|c|c|c|c|c|}
\hline $\begin{array}{c}\text { Chemical } \\
\text { Group }\end{array}$ & Chemical & $\begin{array}{c}\text { Day } \\
\text { BG-11 }\end{array}$ & $\begin{array}{c}\text { Day } \\
\text { MA }\end{array}$ & $\begin{array}{c}\text { p-value } \\
\mathbf{( 9 9 . 5 \% )}\end{array}$ \\
\hline Cyanopeptolin & Cyanopeptolin A & 20 & 15 & $\mathbf{0}$ \\
\hline Cyanopeptolin & Cyanopeptolin C & 20 & 20 & $\mathbf{0}$ \\
\hline Cyanopeptolin & Cyanopeptolin B & 20 & 15 & 0.821 \\
\hline Cyanopeptolin & Cyanopeptolin 900 & 20 & 15 & 1 \\
\hline Cyanopeptolin & Cyanopeptolin 945 & 15 & 15 & $\mathbf{0}$ \\
\hline Cyanopeptolin & Cyanopeptolin 963A & 15 & 15 & 0.953 \\
\hline Cyanopeptolin & Cyanopeptolin 970 & 15 & 15 & 1 \\
\hline Cyanopeptolin & Cyanopeptolin 984 & 15 & 15 & 1 \\
\hline Cyanobactin & Aerucyclamide A & 15 & 15 & $\mathbf{0}$ \\
\hline Cyanobactin & Aerucyclamide C & 15 & 15 & $\mathbf{0 . 0 0 1}$ \\
\hline Cyanobactin & Aerucyclamide B & 15 & 15 & $\mathbf{0}$ \\
\hline Cyanobactin & Aerucyclamide D & 20 & 15 & 0.995 \\
\hline Aeruginosin & Aeruginosin 650 & 15 & 15 & 0.749 \\
\hline Aeruginosin & Aeruginosin 684 & 15 & 15 & $\mathbf{0}$ \\
\hline Microcystin & {$\left[\right.$ Asp ${ }^{3}$ ]-Microcystin-LR } & 20 & 15 & 0.64 \\
\hline Microcystin & Microcystin-LR & 20 & 15 & 0.113 \\
\hline
\end{tabular}

\subsection{2b Media effects on cyanopeptide production by M. flos-aquae CPCC 461}

This strain did not produce many metabolites aside from a few cyanobactin variants and metabolites from an unknown chemical class. The production profile of these metabolites is shown in Figure 3.30 for comparison of relative amounts. 


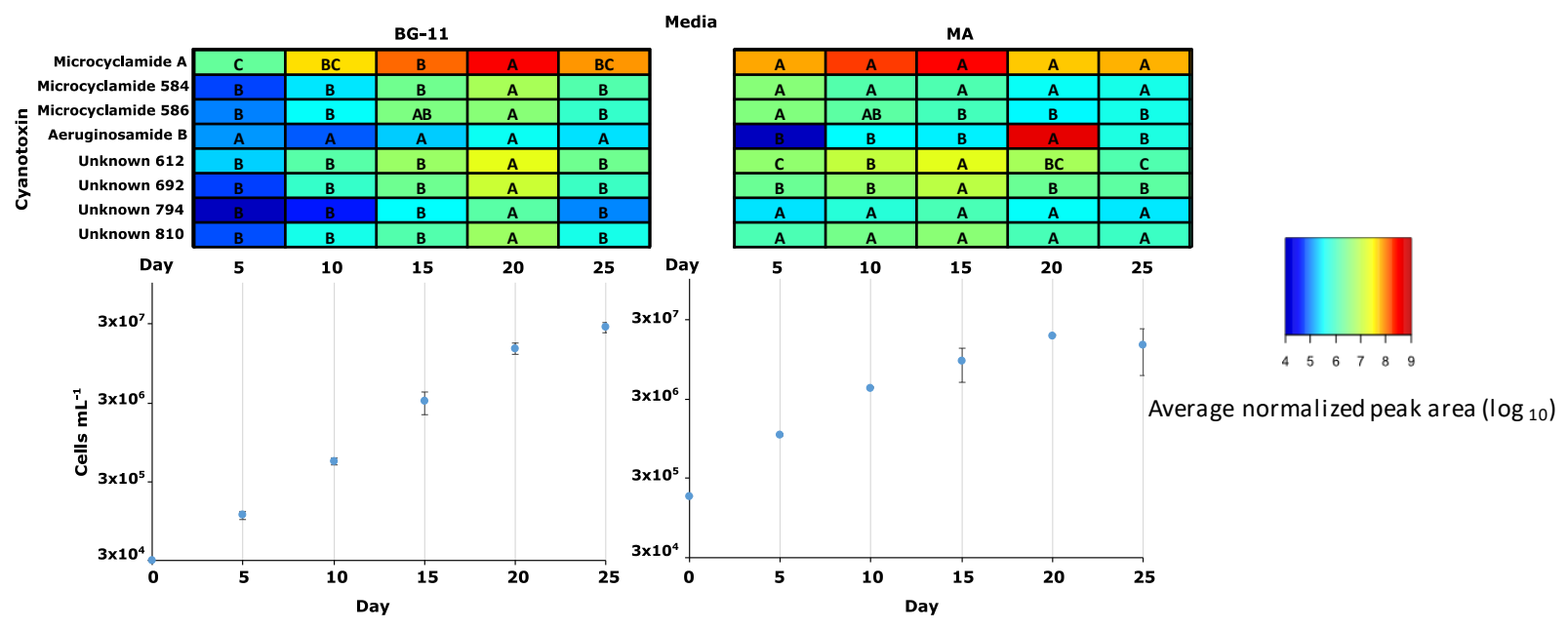

Figure 3.30: Heatmap depicting cyanopeptide production by $M$. flos-aquae CPCC 461 over time in two growth media (BG-11; left and MA;right), generated with the average normalized peak area ( $\log _{10}$, top). Tukey's LSD test distinguished groups designated with letters to illustrate quantitative differences in the individual cyanopeptide production profile (statistically significant, $\mathrm{p}<0.005$ ). Data points with the same letter within a cyanopeptide row are not statistically significant from one another. Cell count measurements are depicted to correlate toxin production with the phase of growth (bottom).

Microcyclamide is the major metabolite produced by this strain and thus has the highest relative production over the time course. It appears to accumulate over time when the strain is grown in BG-11 yet is always present when grown in MA. The other metabolites are present in much smaller quantities. A similar trend is seen where peak intracellular concentration occurs at an earlier time point when grown in MA versus BG-11. Maximum cyanobactin production occurs at day 20 when M. flos-aquae is grown in BG-11 media and at day 10 when grown in MA media. The series of unknown metabolites reach the maximum quantities on day 20 within BG-11 media and on day 15 in MA media. The maximas in metabolite production for both growth media correlate to the late exponential-early stationary phase of growth for M. flos-aquae CPCC 461 (Figure 3.30).

Table 3.15 compares the relative peak area maximum for major metabolites produced by CPCC 461 when grown in two separate culture media, BG-11 and MA. The maxima for all compounds was on day 20 when grown in BG-11 and for aeruginosamide B when grown in MA. The remaining 
metabolites investigated had maximum relative peak area on day 15 when the strain was grown in MA. The peak day for production correlates with the time that the cells are within the exponential phase and capable of producing large amounts of toxin. The two maxima are different for BG-11 and MA, as CPCC 461 has different growth profiles in these media. The outlier, aeruginosamide $\mathrm{B}$, is in statistically higher relative proportions when the strain is grown in MA.

Table 3.15: Maximum relative abundance (green indicates greater peak area) for selected metabolites produced by CPCC 461 when grown in two culture media with an ANOVA pairwise comparison $(\mathrm{p}<0.05)$ to add significance to the media effects. The p-values are bolded for statistically significant differences in relative peak area for the metabolites between the two growth media.

\begin{tabular}{|c|c|c|c|c|}
\hline $\begin{array}{c}\text { Chemical } \\
\text { Group }\end{array}$ & Chemical & Day BG & Day MA & p-value (99.5\%) \\
\hline Cyanbactin & Microcyclamide A & 20 & 15 & 1 \\
\hline Cyanbactin & Microcyclamide 584 & 20 & 15 & 1 \\
\hline Cyanbactin & Microcyclamide 586 & 20 & 15 & 1 \\
\hline Cyanbactin & Aeruginosamide B & 20 & 20 & $\mathbf{0}$ \\
\hline Unknown & Unknown 612 & 20 & 15 & 1 \\
\hline Unknown & Unknown 692 & 20 & 15 & 0.992 \\
\hline Unknown & Unknown 794 & 20 & 15 & 1 \\
\hline Unknown & Unknown 810 & 20 & 15 & 1 \\
\hline
\end{tabular}

\section{$\underline{3.4 .2 c \text { Media effects on cyanopeptide production by M. aeruginosa CPCC } 632}$}

M. aeruginosa CPCC 632 produced a wide range of cyanopeptide groups including anabaenopeptins, microviridins, cyanopeptolins, microginins, and aeruginosins (Figure 3.3). The production profile of a subset of the produced metabolites over a 25 -day time course and when grown in two different culture media is shown in Figure 3.31. 


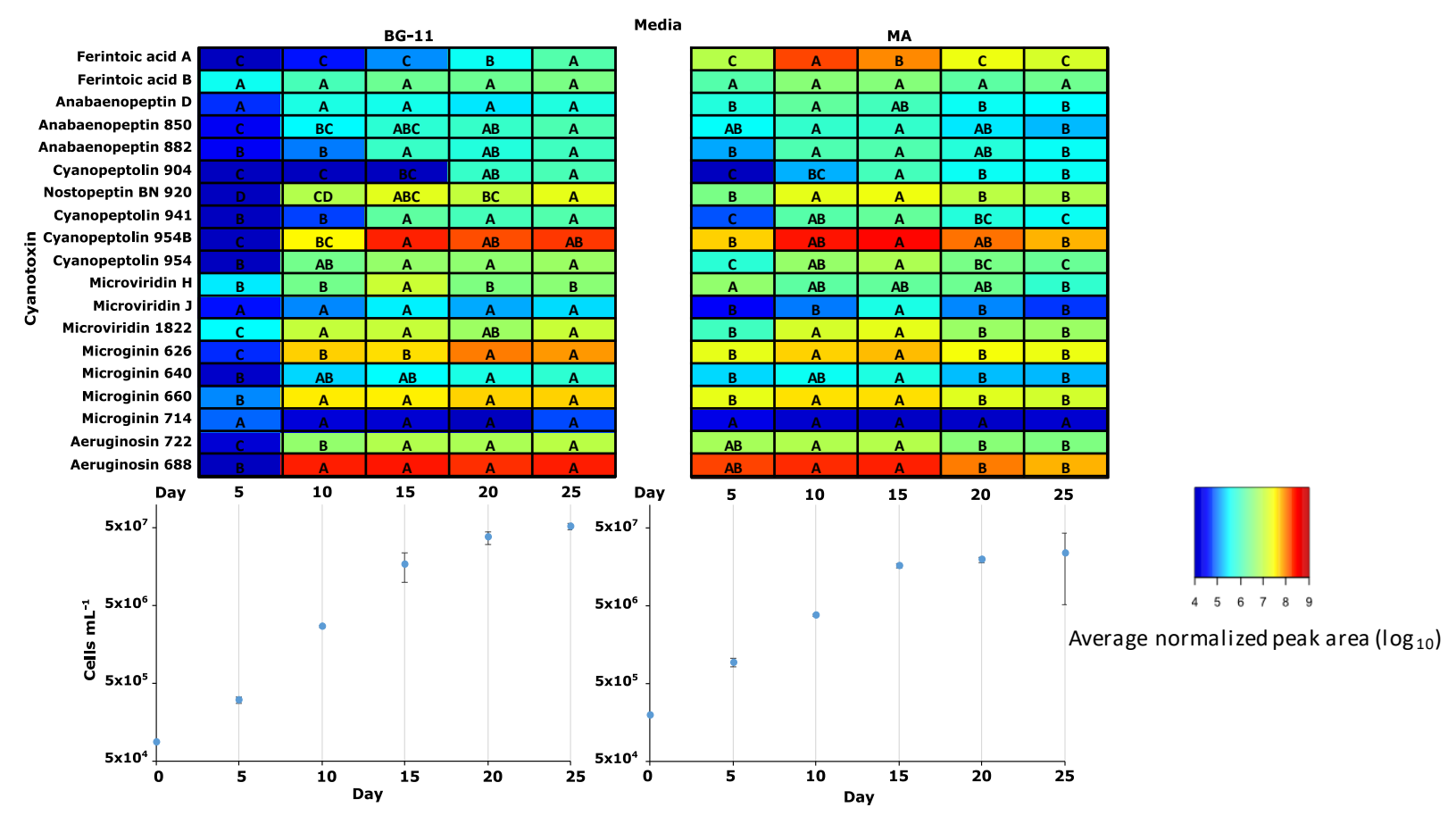

Figure 3.31: Heatmap depicting cyanopeptide production by $M$. aeruginosa CPCC 632 over time in two growth media (BG-11; left and MA; right), generated with the average normalized peak area ( $\log _{10}$, top). Tukey's LSD test distinguished groups designated with letters to illustrate quantitative differences in the individual cyanopeptide production profile (statistically significant, $\mathrm{p}<0.05$ ). Data points with the same letter within a cyanopeptide row are not statistically significant from one another. Cell count measurements are depicted to correlate toxin production with the phase of growth (bottom).

Cyanopeptolin 954B and aeruginosin 688 have the highest relative intracellular concentrations compared to the subset of metabolites depicted with this analysis method. These metabolites are present within the cell for each of the 5 measurements when the strain is grown in MA. The abundance of dark blue data points for the metabolites when the strain is grown in BG-11 suggest that there is very little to no metabolite present within the cell at the first time point. For $M$. aeruginosa CPCC 632, it is apparent that maximum toxin production for each chemical group investigated occurred at the end of the exponential growth phase (Figure 3.31).

Table 3.16 compares the relative peak area maximum for major metabolites produced by CPCC 632 when grown in two separate culture media, BG-11 and MA. Majority of the relative peak area 
maxima are on day 25 when this strain is grown in BG-11 and on day 15 when grown in MA. This correlates with the phase of growth the strain is in when in BG-11 versus MA. The strain has a faster growth rate in MA as it reaches the stationary phase of growth at an earlier time point than when grown in BG-11. This is further seen with peak area maximums for the investigated metabolites in Table 3.16 showing highest relative intracellular concentrations at day 15 for MA and at a later time point for BG-11, day 25. Ferintoic acid A was produced in statistically higher intracellular concentrations when CPCC 632 was grown in MA whereas microginin 626 was present in statistically higher levels when the strain was grown in BG-11.

Table 3.16: Maximum relative abundance (green indicates greater peak area) for selected metabolites produced by CPCC 632 when grown in two culture media with an ANOVA pairwise comparison $(\mathrm{p}<0.05)$ to add significance to the media effects. The $p$-values are bolded for statistically significant differences in relative peak area for the metabolites between the two growth media.

\begin{tabular}{|c|c|c|c|c|}
\hline Chemical Class & Chemical & $\begin{array}{c}\text { Day } \\
\text { BG }\end{array}$ & Day MA & p-value (99.5\%) \\
\hline Anabanopeptin & Ferintoic acid A & 25 & 15 & $\mathbf{0}$ \\
\hline Anabanopeptin & Ferintoic acid B & 25 & 15 & 1 \\
\hline Anabanopeptin & Anabaenopeptin D & 25 & 15 & 1 \\
\hline Anabanopeptin & Anabaenopeptin 850 & 25 & 15 & 1 \\
\hline Anabanopeptin & Anabaenopeptin 882 & 25 & 15 & 1 \\
\hline Cyanopeptolin & Cyanopeptolin 904 & 25 & 15 & 1 \\
\hline Cyanopeptolin & Nostopeptin BN 920 & 25 & 15 & 1 \\
\hline Cyanopeptolin & Cyanopeptolin 941 & 15 & 15 & 1 \\
\hline Cyanopeptolin & Cyanopeptolin 954B & 15 & 15 & 0.314 \\
\hline Cyanopeptolin & Cyanopeptolin 954 & 25 & 15 & 1 \\
\hline Microviridin & Microviridin H & 15 & 20 & 0.559 \\
\hline Microviridin & Microviridin J & 15 & 15 & 1 \\
\hline Microviridin & Microviridin 1822 & 15 & 15 & 0.601 \\
\hline Microginin & Microginin 626 & 20 & 15 & $\mathbf{0}$ \\
\hline Microginin & Microginin 640 & 25 & 15 & 1 \\
\hline Microginin & Microginin 660 & 20 & 15 & 1 \\
\hline Microginin & Microginin 714 & 25 & 20 & 1 \\
\hline Aeruginosin & Aeruginosin 722 & 25 & 15 & 1 \\
\hline Aeruginosin & Aeruginosin 688 & 15 & 15 & 0.418 \\
\hline
\end{tabular}




\subsection{2d Media effects on cyanopeptide production by M. aeruginosa CPCC 633}

This strain produces congeners within the following chemical classes: cyanopeptolin, microviridin, aeruginosamide, microginin, and anabaenopeptin. A few congeners were selected for heatmap analysis for rapid comparison of their production profiles when CPCC 633 is grown in the two culture media, BG-11 and MA (Figure 3.32).

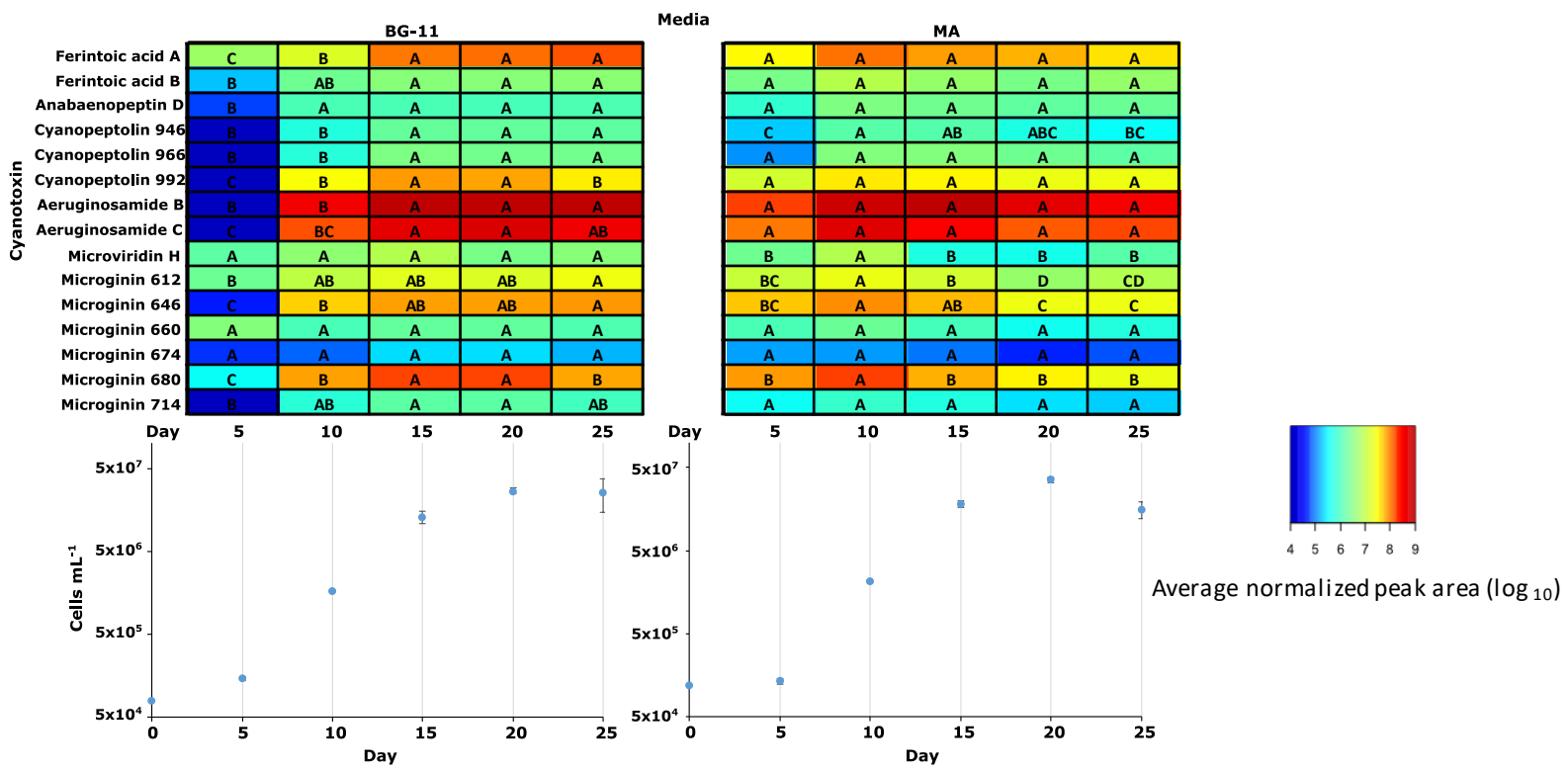

Figure 3.32: Heatmap depicting cyanopeptide production by M. aeruginosa CPCC 633 over time in two growth media (BG-11; left and MA; right), generated with the average normalized peak area $\left(\log _{10}\right.$, top). Tukey's LSD test distinguished groups designated with letters to illustrate quantitative differences in the individual cyanopeptide production profile (statistically significant, $\mathrm{p}<0.05$ ). Data points with the same letter within a cyanopeptide row are not statistically significant from one another. Cell count measurements are depicted to correlate toxin production with the phase of growth (bottom).

The linear cyanobactins, aeruginosamides, have the highest relative intracellular concentration based on heatmap analysis. Ferintoic acid A and microginin 680 are also present in high abundance within the cell in comparison to the other metabolites investigated here. It appears as one metabolite dominates the production for the group and the other congeners within said group are produced at lower levels. Maximum production of the metabolites produced by M. aeruginosa 
CPCC 633 vary based on the growth media (Figure 3.32). Peak production of secondary metabolites when the strain was grown in BG-11 media occur when the cells exit exponential phase of growth and enter stationary phase. When the strain was grown in MA media, peak metabolite production occurs mid-exponential growth phase.

Table 3.17 compares the relative peak area maximum for major metabolites produced by CPCC 633 when grown in two separate culture media, BG-11 and MA. Peak area maxima vary between the compounds investigated when the strain was grown in BG-11 however day 15 appears to be the maximum day for all but one metabolite investigated. Ferintoic acid A and two microginins, 646 and 680 have statistically higher intracellular levels when CPCC 633 was grown in BG-11 media.

Table 3.17: Maximum relative abundance for selected metabolites produced by CPCC 633 when grown in two culture media with an ANOVA pairwise comparison $(\mathrm{p}<0.05)$ to add significance to the media effects. The $p$-values are bolded for statistically significant differences in relative peak area for the metabolites between the two growth media.

\begin{tabular}{|c|c|c|c|c|}
\hline Chemical Class & Chemical & Day BG & Day MA & p-value (99.5\%) \\
\hline Anabaenopeptin & Ferintoic acid A & 25 & 15 & $\mathbf{0 . 0 0 1}$ \\
\hline Anabaenopeptin & Ferintoic acid B & 25 & 15 & 1 \\
\hline Anabaenopeptin & Anabaenopeptin D & 15 & 15 & 1 \\
\hline Cyanopeptolin & Cyanopeptolin 946 & 15 & 15 & 1 \\
\hline Cyanobactin & Cyanopeptolin 966 & 15 & 15 & 1 \\
\hline Cyanobactin & Cyanopeptolin 992 & 15 & 15 & 1 \\
\hline Cyanobactin & Aeruginosamide B & 20 & 15 & 1 \\
\hline Cyanobactin & Aeruginosamide C & 20 & 15 & 0.917 \\
\hline Microviridin & Microviridin H & 15 & 15 & 1 \\
\hline Microginin & Microginin 612 & 25 & 15 & 1 \\
\hline Microginin & Microginin 646 & 25 & 15 & $\mathbf{0 . 0 4 9}$ \\
\hline Microginin & Microginin 660 & 15 & 15 & 1 \\
\hline Microginin & Microginin 674 & 20 & 15 & 1 \\
\hline Microginin & Microginin 680 & 20 & 15 & $\mathbf{0}$ \\
\hline Microginin & Microginin 714 & 20 & 20 & 1 \\
\hline
\end{tabular}




\section{Discussion}

Over the last one hundred years, cyanobacterial blooms have been increasing in both frequency and magnitude. The biological factors that promote cyanobacterial growth and toxin production are broadly understood. The generation and release of cyanotoxins into the environment pose a threat to human and ecosystem health. The majority of research efforts have been invested in MCs due to their potent hepatotoxicity and classification as a possible human carcinogen (IARC group 2B). The chemistry, toxicology, and environmental concentrations of the vast majority of other cyanotoxins are unknown. Untargeted and semi-targeted mass-spectrometry based metabolomics was used to study the strain specific variation in chemical profiles for five Microcystis strains. Target strains have been prioritized for large scale cultivation and isolation of novel cyanotoxins for structural and toxicological characterizations.

\subsection{Untargeted mass-spectrometry based metabolomics to understand strain specific variation between Microcystis species.}

\subsubsection{Discussion of multivariate analyses: PCA and loading plot.}

Microcystis species are known to produce diverse and complex mixtures of cyanopeptides (Janssen, 2019). The PCA analysis visualized which Microcystis strains have similar or different cyanopeptide profiles (Figure 3.1). Supporting the PCA, the factor loading plot distinguished specific features (compounds) characteristic of chemical profiles for the four Microcystis aeruginosa strains (CPCC 299, CPCC 300, CPCC 632, CPCC 633) and one M. flos-aquae (CPCC 461, Figure 3.2). Additionally, a Kruskal Wallis test with Benjamini-Hochberg correction provided statistical significance to the features that were strain specific. 
As these strains are all within the genus Microcystis, it is assumed that there are similarities within the primary metabolism (growth, development, reproduction, (Neilan, Dittmann, Rouhiainen, Bass, Schaub, Sivonen, \& Börner, 1999)). We are viewing the entire intracellular metabolome, which is likely to contain primary metabolites. The loading plot contains many features that did not match databases or spectral libraries, but are central within the loading plot meaning that they are shared metabolites produced by the Microcystis strains. A hypothesis is that these unidentified features are from primary metabolism pathways. To determine which features unite and differentiate the chemotypes, features furthest away from the center of the loading plot were investigated.

The two most similar metabolic profiles are those of M. aeruginosa CPCC 632 and CPCC 633, which share the production of cyanopeptides within the microviridin, microginin, cyanopeptolin, and anaebaenopeptin groups. The triplicate data points for these strains overlap within the PCA plot suggesting a strong agreement in their metabolic profiles (Figure 3.1). Further investigation reveals that similar mixtures of congeners within the microginin and anabaenopeptin cyanopeptide groups are produced by these strains. Variation between M. aeruginosa CPCC 632 and CPCC 633 arise as M. aeruginosa CPCC 632 is capable of producing aeruginosins and M. aeruginosa CPCC 633 cannot produce these metabolites but synthesizes linear cyanobactins. More variation arises with cyanopeptolins as M. aeruginosa CPCC 632 produces congeners with the partial amino acid sequences $\mathrm{NMe}$-Cl-Tyr-Ahp and NMeTyr-Phe-Ahp whereas M. aeruginosa CPCC 633 produces cyanopeptolins with the partial amino acid sequence NMePhe-Phe-Ahp (Table 3.6). Therefore, although there is strong genetic overlap in the NRPS synthase gene clusters for these two strains, differences manifest in the less abundant metabolites produced which distinguish their chemical profiles (Figure 3.3) 
M. aeruginosa CPCC 299 and CPCC 300 have the next closely related metabolome according to the PCA (Figure 3.1, Figure 3.2). These two strains are known "toxic" Microcystis strains as they both produce congeners of the hepatotoxic cyanopeptide group, Microcystins (Racine et al., 2019). Factor loading analysis provides agreement with previous reports that the two MC congeners they produce as well as the aerucyclamide congeners are the same. Yet the congeners of cyanopeptolins produced by M. aeruginosa CPCC 299 and CPCC 300 varied. M. aeruginosa CPCC 299 produced cyanopeptolins with the partial amino sequence NMePhe-Phe-Ahp whereas M. aeruginosa CPCC 300 produced congeners with the partial amino acid sequence NMePhe-Lxx-Ahp. As with $M$. aeruginosa CPCC 632 and CPCC 633, the formerly mentioned strains do not share the production of specific metabolite groups such as aeruginosins by M. aeruginosa CPCC 300 and further the congeners within shared cyanopeptide groups such as cyanopeptolins distinguish their chemical profiles.

The triplicate data points for M. flos-aquae CPCC 461 are isolated within the PCA (Figure 3.1) suggesting the largest difference in total chemical profile when comparing the Microcystis strains. This strain produces few metabolites with the major metabolites being microcyclamides, which are not produced by the M. aeruginosa strains (K. Ishida et al., 2000). A few features that statistically contribute to the variance of the chemical profile for M. flos-aquae CPCC 461 are not previously reported and do not contain any diagnostic features that relate to the other cyanopeptide groups. This depicts the limitations of untargeted LC-MS/MS, as here we can distinguish this strain as significantly different from the $M$. aeruginosa strains and have the $m / z$ and retention time for these compounds, but we are unable to characterize explicitly which chemicals they are. Further analysis, such as genomics or compound isolation, purification, and characterization is required to answer this question. 


\subsubsection{Discussion of the molecular network data for five Microcystis strains.}

Multivariate methods such as PCA and factor loading analysis aid in visually depicting the similarities and differences in chemical profiles between samples. The variables assessed are all the compounds detected that meet the given parameters and can be thousands of compounds, identified only by $m / z$ and retention time. Pairing this method with GNPS molecular networking provides a starting point for identifying compound classes and dereplication of natural products. GNPS molecular networking is a method that utilizes the concept that structurally related chemicals will fragment in similar manners and generate common product ions within the MS/MS. The resulting molecular network has clusters of chemicals that contain common structural features. This method is useful for Microcystis extracts as the majority of their secondary metabolites are peptides that arise from NRPS, NRPS-PKS and RiPP pathways. Further, most cyanopeptide groups share diagnostic structural features including partial amino acid sequences or nonproteinaceous amino acids that result in unique product ions (Table 2.3).

The chemical profiles of the five studied Microcystis strains are significantly different and strain specific metabolites have been identified here (Figure 3.2, Figure 3.3). The untargeted metabolomic strategies applied to their extracts show the metabolic variation with the chemical groups of cyanopeptides that are produced as well as the congeners produced within each class. Furthermore, the molecular network suggests that these strains have different metabolomes but contain various overlapping biosynthetic mechanisms. Of interest is the generation of multiple network clusters for the same chemical group such as microviridins, cyanopeptolins, and cyclic cyanobactins. Multiple network clusters for the same chemical group suggests that there are large enough structural differences between the congeners that results in them clustering separately. This is important as the structural diversity of cyanopeptides is vast and annotation of all congeners 
proves difficult when inspecting a peak list of $m / z$ and retention times alone. Molecular networking provides a place to start with unknown congener annotation, as it gives a gauge of structural similarity of the metabolites to previously identified compounds and allows for in-depth subclassification of cyanopeptides.

Alongside compound identification and dereplication, molecular networking illustrates metabolomic similarities and differences. For example, M. aeruginosa CPCC 299 produces metabolites within the following chemical classes: microviridin, microcystin, cyanobactin, and cyanopeptolins whereas M. aeruginosa CPCC 300 produces metabolites within the following classes: microcystins, cyanobactins, and cyanopeptolins (Figure 3.3). These two strains have an overlap in their biosynthetic potential as they both can make microcystins, cyanobactins, and cyanopeptolins, although M. aeruginosa CPCC 299 has additional biosynthetic genes for the synthesis of microviridins. Microviridins are synthesized by the RiPP pathway (Ziemert et al., 2010), a pathway different than the nRPS pathway the other chemical classes are synthesized by, suggesting M. aeruginosa CPCC 299 has the capacity to utilize another biosynthetic pathway for cyanopeptide production. Dissecting these two chemical profiles further, it is now known that the congeners within these shared chemical classes are different. M. aeruginosa CPCC 299 produces strictly Phe-Ahp containing cyanopeptolins (molecular network cluster \#1) whereas M. aeruginosa CPCC 300 produced strictly Lxx-Ahp containg cyanopeptolins (molecular network cluster \#2, Figure 3.3, (Cornel et al., 1993; Von Elert et al., 2005)). The small variation within the peptide sequence has been differentiated by the diagnostic product ions generated within the MS/MS of known cyanopeptolins within these two subclasses (Figure 3.5). M. aeruginosa CPCC 299 has an NRPS enzyme cluster for cyanopeptolins with specificity for a Phe residue in position 2 with $M$. aeruginosa CPCC 300 having specificity for a Lxx residue in position 2. This minor change in 
enzyme-substrate selectivity generates a whole new subclassification of cyanopeptolins. This variability in enzyme structure and thus amino acid specificity results in the production of hundreds of congeners for each metabolite produced by the NRPS and RiPP pathway (Strieker et al., 2010). RiPP synthesized peptides may have increased structural variation as the identity, number, and location of the post-translational modification is independent of the core peptide synthesis.

An interesting chemotypic difference between these strains visualized within the molecular network is that four of the five Microcystis strains produce metabolites within the cyanobactin group. M. aeruginosa CPCC 299 and CPCC 300 produce cyclic cyanobactins (aerucyclamides, molecular network cluster \#13) whereas M. aeruginosa CPCC 633 produces linear cyanobactins (aeruginosamides, molecular network cluster \#8) and M. flos-aquae CPCC 461 produces both cyclic cyanobactins (microcyclamides, molecular network cluster \#12) and the linear aeruginosamides (Figure 3.3, (K. Ishida et al., 2000; Leikoski et al., 2013; Portmann, Blom, Gademann, et al., 2008; Portmann, Blom, Kaiser, et al., 2008)). Cyanobactins are generated by the nRPS pathway and are cyclized through an enzymatically catalyzed reaction with a carboxy terminal and a terminal amine. M. aeruginosa CPCC 633 and M. flos-aquae CPCC 461 both produce aeruginosamides, which contain a methyl group on the carboxy terminal making $\mathrm{N}$ to $\mathrm{C}$ macrocyclization impossible (Leikoski et al., 2013). An alternative explanation for this is that the enzyme complex responsible for synthesizing these metabolites does not contain a macrocyclase module. The cyclic cyanobactins produced by M. aeruginosa CPCC 299 and CPCC 300, and M. flos-aquae CPCC 461 are termed cyclamides and are broken into two subclasses: microcyclamides and aerucyclamides. M. aeruginosa CPCC 299 and CPCC 300 produce aerucyclamides and M. flos-aquae CPCC 461 produces microcyclamides. The difference between these classes are the 
heterocyclized amino acid moieties and their number within the molecule. The mixture of aerucyclamide congeners produced by M. aeruginosa CPCC 299 and CPCC 300 are the same suggesting conserved biosynthetic genes between these strains. The microcyclamide congeners produced by M. flos-aquae CPCC 461 differ from the aerucyclamides with substitution of the proteinaceous amino acids. Both microcyclamides and aerucyclamides contain three heterocycles, two of which are generated by cyclization of cysteine (thiazole or thiazoline) and one is formed by cyclization of either serine or threonine (oxazole or oxazoline, (Ge et al., 2019)). This supports the hypothesis that $10-30 \%$ of all cyanobacteria have the genetic material for a cyanobactin pathway (Leikoski et al., 2013).

\subsubsection{Discussion of relative cyanopeptide abundance within Microcystis extracts.}

In this study, the relative abundance of metabolites identified within the molecular network and utilized as features within the multivariate analysis are compared by normalizing peak area with biomass. This is an accepted proxy for comparison of relative amounts of metabolites that cannot be quantified with a purified standard (Tiam et al., 2019). Utilizing normalized peak area has advantages as it enables comparison of metabolite "quantities" in reference to other chemicals as well as relative production over time (Figures 3.29-3.32). For MC-LR, absolute quantities in units

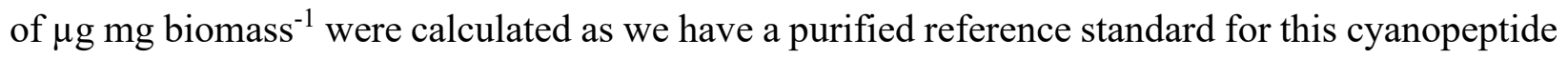
(Figure 3.28, Table 3.13). Quantification of all the other detected cyanopeptides could not be performed due to lack of available reference material.

Within the relative abundance figure (Figure 3.14), a snapshot of the intracellular profile of cyanopeptides on day 15 of growth is visualized for the five Microcystis strains. Varying amounts of cyanopeptides are seen with some metabolites appearing in slightly greater intracellular concentrations over the other congeners. A recent investigation of cyanopeptide co-production 
dynamics elucidated that when growth conditions are varied the proportions of cyanopeptide groups remains constant (Natumi \& Janssen, 2020). This suggests that the mechanisms for cyanopeptide co-production are intertwined and are not related to nutrient requirement changes during the growth cycle.

Within the heatmap analysis, the time point for maximum intracellular metabolite concentrations are in the late exponential phase of growth (Figure 3.29-3.32). Although in some cases, the maximum production varied based on the compound of interest. For example, when M. aeruginosa CPCC 300 was grown in BG-11 maximum aeruginosin 650 concentration occurs at day 10 whereas maximum intracellular concentration of microcystin-LR occurs at day 20 (Figure 3.26). Variables that must be considered are the potential for the metabolite to be exported from the cell, and the total intracellular metabolite concentration would not include excreted metabolites.

There are complications with using peak area as a proxy as these molecules are structurally different, varying in size, cyclic vs linear, as well as amino acid sequences, the extent of ionization varies resulting in differences in peak shape and size. For example, some metabolites may ionize very well and give a large peak area as each molecule has been ionized providing a larger signal. In contrast, a poor ionizing molecule will produce a smaller signal in comparison to how much compound is actually present. Therefore, comparing relative abundance of compounds within the same chemical group makes more sense than comparing between cyanopeptide groups as the structural similarity resulting from the shared chemical features would provide similar ionization capacities.

4.2 Semi-targeted mass-spectrometry based metabolomics for detection of cyanopeptide groups in Microcystis extracts and environmental samples. 


\subsubsection{Discussion of semi-targeted approach for microcystin detection.}

Microcystins are known hepatotoxins with more than 300 congener reports and copious information regarding their toxicity and environmental concentrations. With the number of congener reports steadily increasing, rapid detection and putative identification of microcystin congeners must evolve. The established semi-targeted approach for the detection of Adda containing cyanopeptides was tested with Microcystis extracts and validated with detection of congeners within environmental samples. Interestingly, similar microcystin congener profiles were detected within the M. aeruginosa CPCC 299 and CPCC 300 extracts as well as the environmental samples (Table 3.16-3.17). The identity of the MC-LR and [Asp $\left.{ }^{3}\right]-M C-L R$ congeners was confirmed with purified reference material with putative identification of $\left[\mathrm{Asp}^{3}\right.$, $\left.\mathrm{Dha}^{7}\right]$-MC-LR and MC-LA based on HR-MS and product ion spectra. Previous reports of MC-LA being a persistent microcystin congener within the water column and within tissues of bivalves and fish suggest that production of this congener is advantageous to the producing species since it does not bind to sediment but remains active within the water column and can bioaccumulate within predators (Díez-Quijada et al., 2019).

\subsubsection{Discussion of semi-targeted approach for cyanopeptolin detection.}

To further understand cyanopeptolin diversity produced by the Microcystis strains of interest, DFF plots were generated searching for different possible partial amino acid sequences (Table 3.5). The four M. aeruginosa strains investigated produce different mixtures of cyanopeptolins (CPCC 299, CPCC 300, CPCC 632, and CPCC 633). The subclassification of cyanopeptolins based on partial amino acid sequence derived from the molecular networking aided in the semi-targeted search for cyanopeptolins in both Microcystis cultures and environmental samples. Variation in the partial 
amino acid sequence was great enough to generate two cyanopeptolin molecular network clusters and arose from the residue directly adjacent to the characteristic non-proteinaceous amino acid present in all cyanopeptolins, Ahp. M. aeruginosa CPCC 300 exclusively produced Lxx-Ahp cyanopeptolins as described in the molecular network, with 14 cyanopeptolins detected via the DFF approach which is 6 more congeners than detected within the molecular network. $M$. aeruginosa CPCC 299, CPCC 632, and CPCC 633 produce cyanopeptolin congeners with the partial amino acid sequence Phe-Ahp. Further subclassification occurs between these cyanopeptolin profiles as M. aeruginosa CPCC 299 and CPCC 633 produce exclusively cyanopeptolins with a NMePhe in position 3 whereas M. aeruginosa CPCC 632 produces cyanopeptolins with either $\mathrm{NMeTyr}$ or $\mathrm{NMe}-\mathrm{Cl}-\mathrm{Tyr}$ in position 3. Some cyanopeptolin biosynthesis gene clusters encode a gene for a halogenase enzyme which catalyzes halogenation of specific amino acid residues (Nishizawa et al., 2011). This property explains the coproduction of both chlorinated and non-chlorinated cyanopeptolins by M. aeruginosa CPCC 632 and nonchlorinated cyanopeptolins by the remainder of M. aeruginosa strains. M. flos-aquae CPCC 461 did not produce any detectable cyanopeptolins and none of the studied strains produced cyanopeptolins with the partial amino acid sequence NMeTyr-Lxx-Ahp which accounts for $12 \%$ of all cyanopeptolin congeners according to the generated cyanopeptolin sequence database (Supp Table S2, McDonald et al., 2020).

\subsubsection{Discussion of semi-targeted approach for aeruginosin detection.}

Five aeruginosin congeners were detected and annotated within the molecular network produced by M. aeruginosa CPCC 300 and CPCC 632 (Figure 3.3). The congeners detected within the molecular network generated two subclassifications of aeruginosins based on partial amino acid sequences, Phe-Choi aeruginosins (M. aeruginosa CPCC 300) and Tyr-Choi aeruginosins ( $M$. 
aeruginosa CPCC 632). Diagnostic product ions were identified for the Choi moiety and subsequently used to search Microcystis strains and environmental samples for potential aeruginosins (Table 3.7). The result of the semi-targeted search returned more aeruginosin congeners than shown within the molecular network, five congeners produced by M. aeruginosa CPCC 300 and seven congeners produced by M. aeruginosa CPCC 632 (Table 3.8). Both $M$. aeruginosa CPCC 300 and CPCC 632 display the capability of halogenating the Hpla moiety of aeruginosin congeners, as seen within the structure of aeruginosin 89 reported from Microcystis species (Welker et al., 2006). This is visible within the full scan MS by the isotopic abundance of the $\mathrm{M}+2$ peak. Investigating the MS/MS data for the additional aeruginosin congeners detected by DFF revealed the missing link between the molecular network data and the DFF data. Two congeners containing the partial amino acid sequence Lxx-Choi and three containing Phe-Choi did not meet the molecular networking requirements for product ion spectra similarity and thus were not present or clustered within the network. Also not depicted within the molecular network is the overlap in production of As 609 and the sulfated version As 689 by M. aeruginosa CPCC 300 and M. aeruginosa CPCC 632. Instead, it is visualized that $M$. aeruginosa CPCC 632 produces these compounds and $M$. aeruginosa $\mathrm{CPCC} 300$ does not. The levels of the aeruginosin congeners within the M. aeruginosa CPCC 300 extract may be low and resulted in poor fragmentation or noninclusion within the molecular network.

\subsubsection{Discussion of semi-targeted approach for microginin detection.}

Cyanopeptides within the microginin group have few common and diagnostic product ions as the N-terminal acyl chain derived from a PKS synthase pathway can have many modifications such as chain length, halogenation, and/or methylation. The remainder of the molecule is highly variable based on a structural database of all previously reported microginin congeners (Supp. Table S3). 
For detection of these congeners via a semi-targeted approach, a filter within the DFF search must be used. This encapsulates the different possible N-terminal acyl chain variations that are biosynthesized (Table 3.9).

Two of the five investigated strains produced different mixtures of microginin congeners, $M$. aeruginosa $\mathrm{CPCC} 632$ and CPCC 633. Based on DFF analysis for microginins, M. aeruginosa CPCC 632 produces congeners with exclusively an eight carbon chain $\mathrm{N}$-terminal chain variants (R-Ahoa) whereas M. aeruginosa CPCC 633 produces both eight carbon chain and ten carbon chain N-terminal groups (R-Ahoa, R-Ahda, Table 3.10). Methylation of the amino group on the $\mathrm{N}$-terminal chain was more commonly seen within the microginin congeners produced by $M$. aeruginosa CPCC 632.

The majority of previously reported microginin congeners contain the Ahda N-terminal moiety with variable chlorination and methylation, with incorporation of Ahoa and its variants as the Nterminal moiety being extremely rare (Strangman \& Wright, 2016). This is proven with the detection of thirteen R-Ahda containing microginins within the environmental samples and only six R-Ahoa containing microginins (Table 3.8). Possible underreporting of R-Ahoa containing microginins may have occurred in previous reports due to lack of purified standards and few characterized congeners. These seem like rare new compounds worth pursuing.

\subsubsection{Optimizing cyanopeptide detection and identification.}

It has been shown here that the Microcystis genus is a prolific source of peptide natural products with vast structural diversity. Most research efforts are towards the hepatotoxicmicrocystins, with little information surrounding the toxicity, fate, and environmental concentrations of the other cyanopeptide classes. As cyanobacterial blooms escalate in their occurrence and severity, it is 
believed the complexity of the cyanopeptides that are produced will escalate as well (Tiam et al., 2019). Rapid detection, identification, and toxicological assessment of the cyanopeptides is required to ensure the safety of the general public and the environment.

There are advantages and disadvantages of untargeted metabolomics when attempting to understand and describe the chemical diversity within and between samples. A large advantage is gathering chemical information for thousands of chemicals within a sample. A factor that must be fine-tuned is the chromatography prior to mass analysis as the data dependent acquisition method utilizes the abundance of ions to select which molecules will be fragmented. If you have a large number of chemicals eluting within a small period of time, the most abundant compounds will be selected for fragmentation and the lower abundance compounds will not be acknowledged. A major challenge of operating with large data sets is the processing and interpretation of the data. Without large and comprehensive databases/libraries to describe the chemicals that may be present within the sample, the chemicals that are detected cannot be annotated or assigned an identity. As this approach becomes more widely used, such databases are being compiled and readily accessible on public websites (Strangman et al., 2018; Tiam et al., 2019). With high resolution MS data, concluding on a molecular formula is possible and aids in matching a molecule to literature. As well, matching fragmentation patterns to key structural features within a molecule will help confirm an identity. But when identifying new congeners of a chemical, one cannot conclude on the chemical structure without isolation, purification, and NMR characterization of the compound. But this technique can supply a place to start for said isolation.

Here we have combined an untargeted LC-MS/MS approach with multivariate analysis and semitargeted searches for rapid identification and annotation of different cyanopeptide groups. It was seen here that molecular networking is not a perfect technique with underreporting of the number 
of congeners present within a sample based on follow-up DFF analysis. But the development of the DFF approach was reliant on the data gathered from the molecular networking as a starting point for compound classification. Molecular networking provides vital information for compound identification and classification in a dataset comprised of thousands of compounds. Understanding the molecular features of the cyanopeptides present within a sample can help with potential new compound identification as well as target cyanobacterial strains for large scale cultivation and cyanopeptide purification, for structural and toxicological characterizations.

Confirmation of a metabolites identity and quantification cannot be done without a purified standard. MC-LR and $\left[\mathrm{Asp}^{3}\right]-\mathrm{MC}-\mathrm{LR}$ are the only metabolites identified within this study that have purified standards accessible and thus intracellular concentrations could be quantified (Figure 3.28). These metabolites have undergone extensive toxicological assessment and are high priority cyanopeptides due to their hepatotoxicity. It is seen here that these two metabolites are not the only compounds produced by the two "toxic" Microcystis strains, CPCC 299 and CPCC 300. More studies are required for the isolation and structural characterization of congeners within the other identified chemical groups such as anabaenopeptins which are known (and less studied) PP2A inhibitors (Spoof et al., 2016). Hypotheses can be made regarding the toxicity of a metabolite based on structural motifs and structural similarities to compounds that have been used in pharmacokinetic and pharmacodynamic studies. But with tangible material, unambiguous characterization of toxicokinetics can be done as well as quantification of intra/extra-cellular and environmental concentrations.

\subsection{Media composition effects on cyanopeptide production by Microcystis strains.}

To assess media composition effects on cyanopeptide production, biological parameters must be considered as toxin production is closely correlated with photosynthetic rates (Walls et al., 2018). 
Prior to media composition changes, it was evident that growth rates and physiology of the five Microcystis strains varied (Table 3.11). The phenotypic changes, such as the extent of their green colour suggesting changes in pigment ratios as well as the formation of biofilms resulting in thick mats suggest that the chemical profile differences of the Microcystis strains is a reflection of physiological differences.

Bacterial growth rates were calculated with cell $\mathrm{mL}^{-1}$ for the five Microcystis strains when grown in media containing different concentrations and sources of essential nutrients. Table 3.11 clearly depicts that MA media resulted in the lowest growth rate for all strains, ranging from 0.103-0.216 days $^{-1}$. It has been previously reported that reduction of nutrients $(\mathrm{N}, \mathrm{P}$, micronutrients $(\mathrm{Fe}, \mathrm{Zn}$, etc.)) results in a significant reduction of growth but only reductions in phosphorous or micronutrients resulted in a significant reduction in total cyanopeptide quota (Natumi \& Janssen, 2020). Thus, the changes in growth seen within the Microcystis strains when grown in MA versus BG-11 media could be due to the complex phosphorous source, $\beta$-Na2-glycerophosphate, and a smaller total phosphorous availability.

In our analyses, we compared maximum relative peak area for select metabolites to gage shifts in metabolite production based on media composition over a 25 -day time course. Based on the heatmap analysis, the metabolites that were produced in the largest quantities remained the same suggesting that total intracellular cyanopeptide quota did not shift significantly when the strains were grown in the two pre-defined culture media. Interestingly, when the Microcystis strains were grown in MA medium, the maximum relative intracellular toxin levels occurred within the exponential phase of growth suggesting that when the cells enter the stationary phase of growth cell lysis occurs and the cell-bound toxin levels are released suggesting unfavourable growth conditions (Figures 3.29-3.32, Walls et al., 2018). Whereas when the Microcystis strains were 
grown in BG-11, it appears that the toxin levels continually accumulate with maximum relative peak area occurring when the cells enter the stationary phase of growth.

Contrary to previous reports, a profound media effect is observed on the production of metabolites by M. flos-aquae CPCC 461. When the strain is grown in BG-11 media, the dominant cyanobactin is the cyclic microcyclamide A whereas when the strain is grown in MA media, the dominant cyanobactin is the linear aeruginosamide B (Figure 3.30). There is a large growth rate shift for $M$. flos-aquae CPCC 461 when grown in BG-11 (0.309) and MA (0.103, Table 3.11) suggesting that the shift from cyclic cyanobactins to linear cyanobactins is more energetically favourable when the cells are not growing efficiently. A hypothesis for this is the capacity for the strain to utilize the complex phosphorous source ( $\beta-\mathrm{Na}_{2}$-glycerophosphate).

\subsection{Discussion of the toxicity of the five Microcystis strains.}

Growth of Microcystis species in waterbodies used for recreational purposes or as drinking water resources are of public health concern because of the ability to produce hepatototxins (Pick, 2016). Hepatotoxin-producing strains of Microcystis are classified as toxigenic strains due to protein phosphatase inhibition whereas those without hepatotoxic capabilities are classified as nontoxigenic strains. The cyanopeptide groups with protein phosphatase activity are microcystins and nodularins, and anabaenopeptins (Urrutia-Cordero et al., 2013; Zurawell et al., 2005). In this study, two of the five Microcystis strains are considered toxigenic (M. aeruginosa CPCC 299 and CPCC 300) as they can biosynthesize microcystin congeners. The toxicity of all five strains was assessed with a PP2A inhibition assay (Table 3.11). The strains classified as toxigenic displayed PP2A activity with calculated microcystin equivalents at $0.71 \mu \mathrm{g} \mathrm{L}{ }^{-1}$ for M. aeruginosa CPCC 299 and $0.29 \mu \mathrm{g} \mathrm{L}^{-1}$ for $M$. aeruginosa CPCC 300. Unexpectedly, M. flos-aquae CPCC 461 displayed PP2A inhibition with microcystin equivalents calculated at $0.82 \mu \mathrm{g} \mathrm{L}^{-1}$, larger than both the 
toxigenic strains. Upon screening M. flos-aquae CPCC 461 for Adda-containing metabolites with our semi-targeted approach, no potential microcystins, nor nodularins were detected. The metabolites annotated here produced by the M. flos-aquae CPCC 461 strain are cyanobactins (microcyclamides) which have cytotoxic and antimicrobial activity that cannot be attributed to the protein phosphatase inhibition (K. Ishida et al., 2000). Previous reports of a M. flos-aquae strain show strong inhibition of freshwater amoeba as a result of anabaenopeptin variants (UrrutiaCordero et al., 2013). Potentially, low abundant anabaenopeptins are produced by this strain and were not annotated within the molecular network due to little structural similarity with other metabolites. A screening method for anabaenopeptins via determination of diagnostic product ions for this chemical class would aid in the rapid identification of anabaenopeptin variants.

The non-toxigenic strains (M. aeruginosa CPCC 632 and CPCC 633) produce anabaenopeptin congeners (Figure 3.3, Table S1). Without any displayed PP2A toxicitiy (Table 3.11), it is assumed that the produced variants are either produced in low abundance or have very little activity against PP2A specifically. Other cyanopeptide groups produced by these non-toxigenic strains are cyanopeptolins, microviridins, microginins, aeruginosins, and cyanobactins. Variants within these cyanopeptide groups have bioactive properties such as protease inhibition and present therapeutic potential with anticancer, antifungal, antibacterial, antiviral, and potent enzyme-inhibiting properties. Large scale cultivation of target Microcystis strains for cyanopeptide isolation for structural and toxicological assessment would reveal the changes in toxicokinetics from amino acid substitutions, halogenation, methylation, and sulfonation of the general cyanopeptide group core structures. Microcystis strains provide vast chemical diversity of natural products with various biological activities that are attractive potential drug candidates and therapeutics. 


\section{Conclusion}

The use of untargeted and semi-targeted mass-spectrometry based metabolomics to describe the strain specific cyanopeptide profiles was described here. The metabolome differences of four Microcystis aeruginosa strains (CPCC 299, CPCC 300, CPCC 632, CPCC 633) and one M. flosaquae strain (CPCC 461) were deciphered with the use of untargeted and semi-targeted massspectrometry based metabolomic techniques. Strain specific profiles were elucidated as the coproduction of multiple cyanopeptide groups and characteristic variants. These profiles were compared and visualized through GNPS molecular networking and diagnostic fragmentation filtering analyses. Information gathered from the untargeted techniques was used for the rapid identification of microcystins, cyanopeptolins, aeruginosins, and microginins within environmental samples. Changes in total chemical profile were assessed after alterations to culture media composition. The largest effect was seen in the growth rate which altered the production profiles of the cyanopeptides over time monitored with relative abundances of the metabolites. The production profile analysis provides insight for large scale cultivation of Microcystis strains for the optimal production and isolation of cyanopeptides. Toxicity of the Microcystis extracts was assessed with PP2A inhibition assays which provided agreement with the classification of the toxigenic strains M. aeruginosa CPCC 299 and CPCC 300, and surprising toxicity of the nontoxigenic strain M. flos-aquae CPCC 461. Further investigation for the structural and toxicological characterization of the less studied metabolites produced by Microcystis could reveal potential new bioactive metabolites.

\section{References}

Ackerley, D. F., \& Lamont, I. L. (2003). Substrate Specificity of the Nonribosomal Peptide Synthetase PvdD from. Society, 185(9), 2848-2855. https://doi.org/10.1128/JB.185.9.2848 
Al-Sammak, M. A., Rogers, D. G., \& Hoagland, K. D. (2015). Acute $\beta$ - N -Methylamino-Lalanine Toxicity in a Mouse Model. Journal of Toxicology, 2015. https://doi.org/10.1155/2015/739746

Beger, R. (2013). A Review of Applications of Metabolomics in Cancer. Metabolites, 3(3), 552574. https://doi.org/10.3390/metabo3030552

Bister, B., Keller, S., Baumann, H. I., Nicholson, G., Weist, S., Jung, G., Süssmuth, R. D., \& Jüttner, F. (2004). Cyanopeptolin 963A, a chymotrypsin inhibitor of Microcystis PCC 7806. Journal of Natural Products, 67(10), 1755-1757. https://doi.org/10.1021/np049828f

Botana, L. M., Alfonso, A., Botana, L. M., \& Alfonso, A. (2015). Phycotoxins : Chemistry and Biochemistry. John Wiley \& Sons, Incorporated. http://ebookcentral.proquest.com/lib/oculcarleton-ebooks/detail.action?docID=1895469

Chernoff, N., Hill, D., Lang, J., Schmid, J., Le, T., Farthing, A., \& Huang, H. (2020). The comparative toxicity of 10 microcystin congeners administered orally to mice: Clinical effects and organ toxicity. Toxins, 12(6), 1-18. https://doi.org/10.3390/toxins12060403

Cornel, M., Lukas, O., Tomio, I., Königc, W. A., Michael, B. H., \& Jurgen, W. (1993). Cyanopeptolins, new depsipeptides from the cyanobacteriummicrocystis sp. pcc 7806. The Journal of Antibiotics, 46(10), 1550-1556. https://doi.org/10.7164/antibiotics.46.1550

De Figueiredo, D. R., Azeiteiro, U. M., Esteves, S. M., Gonçalves, F. J. M., \& Pereira, M. J. (2004). Microcystin-producing blooms - A serious global public health issue. Ecotoxicology and Environmental Safety, 59(2), 151-163. https://doi.org/10.1016/j.ecoenv.2004.04.006

Demain, A. L., \& Fang, A. (2000). The natural functions of secondary metabolites. Advances in Biochemical Engineering/Biotechnology, 69, 1-39. https://doi.org/10.1007/3-540-44964$7 \_1$

Demain, Arnold L. (2014). Importance of microbial natural products and the need to revitalize their discovery. Journal of Industrial Microbiology and Biotechnology, 41(2), 185-201. https://doi.org/10.1007/s10295-013-1325-z

Dettmer, K., Aronov, P. A., \& Hammock, B. D. (2007). Mass spectrometry-based metabolomics. Mass Spectrometry Reviews, 26(1), 51-78. https://doi.org/10.1002/mas.20108

Díez-Quijada, L., Prieto, A. I., Guzmán-Guillén, R., Jos, A., \& Cameán, A. M. (2019). Occurrence and toxicity of microcystin congeners other than MC-LR and MC-RR: A review. Food and Chemical Toxicology, 125(December 2018), 106-132. https://doi.org/10.1016/j.fct.2018.12.042

Eliana Henao, Piotr Rzymski, and M. N. W. (2020). Paleolimnological Research : Current Knowledge and. Toxins, 1-15.

Elkobi-Peer, S., \& Carmeli, S. (2015). New prenylated aeruginosin, microphycin, anabaenopeptin and micropeptin analogues from a Microcystis bloom material collected in Kibbutz Kfar Blum, Israel. Marine Drugs, 13(4), 2347-2375. https://doi.org/10.3390/md13042347

Feurstein, D., Kleinteich, J., Heussner, A. H., Stemmer, K., \& Dietrich, D. R. (2010). 
Investigation of microcystin congener-dependent uptake into primary murine neurons. Environmental Health Perspectives, 118(10), 1370-1375.

https://doi.org/10.1289/ehp.0901289

Ge, Y., Czekster, C. M., Miller, O. K., Botting, C. H., Schwarz-Linek, U., \& Naismith, J. H. (2019). Insights into the Mechanism of the Cyanobactin Heterocyclase Enzyme. Biochemistry. https://doi.org/10.1021/acs.biochem.9b00084

Harke, M. J., Steffen, M. M., Gobler, C. J., Otten, T. G., Wilhelm, S. W., Wood, S. A., \& Paerl, H. W. (2016). A review of the global ecology, genomics, and biogeography of the toxic cyanobacterium, Microcystis spp. Harmful Algae, 54, 4-20. https://doi.org/10.1016/j.hal.2015.12.007

Hollingdale, C., Thomas, K., Lewis, N., Békri, K., McCarron, P., \& Quilliam, M. A. (2015). Feasibility study on production of a matrix reference material for cyanobacterial toxins. Analytical and Bioanalytical Chemistry, 407(18), 5353-5363. https://doi.org/10.1007/s00216-015-8695-1

Huang, I., \& Zimba, P. V. (2019). Cyanobacterial bioactive metabolites - A review of their chemistry and biology. Harmful Algae, 83(February), 42-94. https://doi.org/10.1016/j.hal.2018.11.008

Ishida, K., Nakagawa, H., \& Murakami, M. (2000). Microcyclamide, a cytotoxic cyclic hexapeptide from the cyanobacterium Microcystis aeruginosa. Journal of Natural Products, 63(9), 1315-1317. https://doi.org/10.1021/np000159p

Ishida, Keishi, Kato, T., Murakami, M., Watanabe, M., \& Watanabe, M. F. (2000). Microginins , Zinc Metalloproteases Inhibitors from the Cyanobacterium Microcystis aeruginosa. 56, 8643-8656.

Janssen, E. M. L. (2019). Cyanobacterial peptides beyond microcystins - A review on cooccurrence, toxicity, and challenges for risk assessment. Water Research, 151, 488-499. https://doi.org/10.1016/j.watres.2018.12.048

Jones, M., Pinto, E., Torres, M., Dörr, F., Mazur-Marzec, H., Szubert, K., Tartaglione, L., Dell'Aversano, C., Miles, C., Beach, D., McCarron, P., Sivonen, K., Fewer, D., Jokela, J., \& Janssen, E. (2020). Comprehensive database of secondary metabolites from cyanobacteria. BioRxiv - the Preprint Server for Biology, C, 1-16. https://doi.org/10.1101/2020.04.16.038703

Le Manach, S., Duval, C., Marie, A., Djediat, C., Catherine, A., Edery, M., Bernard, C., \& Marie, B. (2019). Global metabolomic characterizations of microcystis spp. Highlights clonal diversity in natural bloom-forming populations and expands metabolite structural diversity. Frontiers in Microbiology, 10(MAR), 1-16. https://doi.org/10.3389/fmicb.2019.00791

Leikoski, N., Liu, L., Jokela, J., Wahlsten, M., Gugger, M., Calteau, A., Permi, P., Kerfeld, C. A., Sivonen, K., \& Fewer, D. P. (2013). Genome mining expands the chemical diversity of the cyanobactin family to include highly modified linear peptides. Chemistry and Biology, 20(8), 1033-1043. https://doi.org/10.1016/j.chembiol.2013.06.015 
Linington, R. G., Edwards, D. J., Shuman, C. F., McPhail, K. L., Matainaho, T., \& Gerwick, W. H. (2008). Symplocamide A, a potent cytotoxin and chymotrypsin inhibitor from the marine Cyanobacterium Symploca sp. Journal of Natural Products, 71(1), 22-27. https://doi.org/10.1021/np070280x

Lodin-Friedman, A., \& Carmeli, S. (2018). Microginins from a microcystis sp. Bloom material collected from the kishon reservoir, Israel. Marine Drugs, 16(3). https://doi.org/10.3390/md16030078

McDonald, K. P., Renaud, J. B., Pick, F. R., Miller, J. D., Sumarah, M. W., \& McMullin, D. R. (2020). Diagnostic Fragmentation Filtering for Cyanopeptolin Detection. Environmental Toxicology and Chemistry.

McKindles, K. M., Manes, M. A., DeMarco, J. R., McClure, A., McKay, R. M., Davis, T. W., \& Bullerjahn, G. S. (2020). Dissolved Microcystin Release Coincident with Lysis of a Bloom Dominated by Microcystis spp. in Western Lake Erie Attributed to a Novel Cyanophage. Applied and Environmental Microbiology, 86(22), 1-14. https://doi.org/10.1128/AEM.01397-20

McMullin, D. R., Hoogstra, S., McDonald, K. P., Sumarah, M. W., \& Renaud, J. B. (2019). Natural Product Discovery with LC-MS/MS Diagnostic Fragmentation Filtering: Application for Microcystin Analysis. Journal of Visualized Experiments.

Méjean, A., Paci, G., Gautier, V., \& Ploux, O. (2014). Biosynthesis of anatoxin-a and analogues (anatoxins) in cyanobacteria. Toxicon, 91, 15-22. https://doi.org/10.1016/j.toxicon.2014.07.016

Nagana Gowda, G. A., \& Djukovic, D. (2014). Overview of mass spectrometry-based metabolomics: Opportunities and challenges. Methods in Molecular Biology, 1198, 3-12. https://doi.org/10.1007/978-1-4939-1258-2_1

Natumi, R., \& Janssen, E. M. L. (2020). Cyanopeptide Co-Production Dynamics beyond Mirocystins and Effects of Growth Stages and Nutrient Availability. Environmental Science and Technology, 54(10), 6063-6072. https://doi.org/10.1021/acs.est.9b07334

Neilan, B. A., Dittmann, E., Rouhiainen, L., Bass, R. A., Schaub, V., Sivonen, K., \& Börner, T. (1999). Nonribosomal peptide synthesis and toxigenicity of cyanobacteria. Journal of Bacteriology, 181(13), 4089-4097. https://doi.org/10.1128/jb.181.13.4089-4097.1999

Neilan, B. A., Dittmann, E., Rouhiainen, L. E. O., Bass, R. A., Schaub, V., Sivonen, K., \& Bo, T. (1999). Nonribosomal Peptide Synthesis and Toxigenicity of Cyanobacteria. 181(13), 40894097.

Nishizawa, T., Ueda, A., Nakano, T., Nishizawa, A., Miura, T., Asayama, M., Fujii, K., Harada, K., \& Shirai, M. (2011). Characterization of the locus of genes encoding enzymes producing heptadepsipeptide micropeptin in the unicellular cyanobacterium Microcystis. The Journal of Biochemistry, 149(4), 475-485. https://doi.org/10.1093/jb/mvq150

Paerl, H. W., \& Millie, D. F. (1996). Physiological ecology of toxic aquatic cyanobacteria. Phycologia, 35(SUPPL.), 160-167. https://doi.org/10.2216/i0031-8884-35-6s-160.1

Paerl, H. W., \& Otten, T. G. (2013). Harmful Cyanobacterial Blooms: Causes, Consequences, 
and Controls. Microbial Ecology, 65(4), 995-1010. https://doi.org/10.1007/s00248-0120159-y

Pick, F. R. (2016). Blooming algae: A Canadian perspective on the rise of toxic cyanobacteria. Canadian Journal of Fisheries and Aquatic Sciences, 73(7), 1149-1158. https://doi.org/10.1139/cjfas-2015-0470

Portmann, C., Blom, J. F., Gademann, K., \& Jüttner, F. (2008). Aerucyclamides A and B: Isolation and synthesis of toxic ribosomal heterocyclic peptides from the cyanobacterium Microcystis aeruginosa PCC 7806. Journal of Natural Products, 71(7), 1193-1196. https://doi.org/10.1021/np800118g

Portmann, C., Blom, J. F., Kaiser, M., Brun, R., Jüttner, F., \& Gademann, K. (2008). Isolation of aerucyclamides $\mathrm{C}$ and $\mathrm{D}$ and structure revision of microcyclamide 7806A: Heterocyclic ribosomal peptides from Microcystis aeruginosa PCC 7806 and their antiparasite evaluation. Journal of Natural Products, 71(11), 1891-1896. https://doi.org/10.1021/np800409z

Racine, M., Saleem, A., \& Pick, F. R. (2019). Metabolome variation between strains of microcystis aeruginosa by untargeted mass spectrometry. Toxins, 11(12). https://doi.org/10.3390/toxins11120723

Rapala, J., Sivonen, K., Lyra, C., \& Niemelä, S. I. (1997). Variation of microcystins in anabaena spp as a function of growth stimuli. Applied and Environmental Microbiology, 63(6), 22062212.

Rohrlack, T., Christoffersen, K., Hansen, P. E., Zhang, W., Czarnecki, O., Henning, M., Fastner, J., Erhard, M., Neilan, B. A., \& Kaebernick, M. (2003). Isolation, characterization, and quantitative analysis of microviridin J, a new Microcystis metabolite toxic to Daphnia. Journal of Chemical Ecology, 29(8), 1757-1770. https://doi.org/10.1023/A:1024889925732

Schindler, D. W., \& Fee, E. J. (1974). Experimental Lakes Area: Whole-Lake Experiments in Eutrophication. Journal of the Fisheries Research Board of Canada, 31(5), 937-953. https://doi.org/10.1139/f74-110

Smith, D. J., \& Underwood, G. J. C. (2000). The production of extracellular carbohydrates by estuarine benthic diatoms: The effects of growth phase and light and dark treatment. Journal of Phycology, 36(2), 321-333. https://doi.org/10.1046/j.1529-8817.2000.99148.x

Spoof, L., Błaszczyk, A., Meriluoto, J., Cegłowska, M., \& Mazur-Marzec, H. (2016). Structures and activity of new anabaenopeptins produced by Baltic Sea cyanobacteria. Marine Drugs, 14(1), 1-14. https://doi.org/10.3390/md14010008

Stewart, A. K., Ravindra, R., Van Wagoner, R. M., \& Wright, J. L. C. (2018). MetabolomicsGuided Discovery of Microginin Peptides from Cultures of the Cyanobacterium Microcystis aeruginosa. Journal of Natural Products, 81(2), 349-355. https://doi.org/10.1021/acs.jnatprod.7b00829

Strangman, W. K., Stewart, A. K., Herring, M. C., \& Wright, J. L. C. (2018). Identification of the new chymotrypsin inhibitor micropeptin 996 by metabolomics-guided analysis. Tetrahedron Letters, 59(10), 934-937. https://doi.org/10.1016/j.tetlet.2018.01.087 
Strangman, W. K., \& Wright, J. L. C. (2016). Microginins 680, 646, and 612 - New chlorinated Ahoa-containing peptides from a strain of cultured Microcystis aeruginosa. Tetrahedron Letters, 57(16), 1801-1803. https://doi.org/10.1016/j.tetlet.2016.03.039

Strieker, M., Tanović, A., \& Marahiel, M. A. (2010). Nonribosomal peptide synthetases: Structures and dynamics. Current Opinion in Structural Biology, 20(2), 234-240. https://doi.org/10.1016/j.sbi.2010.01.009

Süssmuth, R. D., \& Mainz, A. (2017). Nonribosomal Peptide Synthesis-Principles and Prospects. Angewandte Chemie - International Edition, 56(14), 3770-3821. https://doi.org/10.1002/anie.201609079

Tan, V. X., Lassus, B., Lim, C. K., Tixador, P., Courte, J., Bessede, A., Guillemin, G. J., \& Peyrin, J. M. (2018). Neurotoxicity of the Cyanotoxin BMAA Through Axonal Degeneration and Intercellular Spreading. Neurotoxicity Research, 33(1), 62-75. https://doi.org/10.1007/s12640-017-9790-1

Testai, E., Scardala, S., Vichi, S., Buratti, F. M., \& Funari, E. (2016). Risk to human health associated with the environmental occurrence of cyanobacterial neurotoxic alkaloids anatoxins and saxitoxins. Critical Reviews in Toxicology, 46(5), 385-419. https://doi.org/10.3109/10408444.2015.1137865

Tiam, S. K., Gugger, M., Demay, J., Le Manach, S., Duval, C., Bernard, C., \& Marie, B. (2019). Insights into the diversity of secondary metabolites of Planktothrix using a biphasic approach combining global genomics and metabolomics. Toxins, 11(9). https://doi.org/10.3390/toxins1 1090498

Tillett, D., Dittmann, E., Erhard, M., Von Döhren, H., Börner, T., \& Neilan, B. A. (2000). Structural organization of microcystin biosynthesis in Microcystis aeruginosa PCC7806: An integrated peptide-polyketide synthetase system. Chemistry and Biology, 7(10), 753-764. https://doi.org/10.1016/S1074-5521(00)00021-1

Urrutia-Cordero, P., Agha, R., Cirés, S., Lezcano, M. ángeles, Sánchez-Contreras, M., Waara, K. O., Utkilen, H., \& Quesada, A. (2013). Effects of harmful cyanobacteria on the freshwater pathogenic free-living amoeba Acanthamoeba castellanii. Aquatic Toxicology, 130-131, 917. https://doi.org/10.1016/j.aquatox.2012.12.019

Von Elert, E., Oberer, L., Merkel, P., Huhn, T., \& Blom, J. F. (2005). Cyanopeptolin 954, a chlorine-containing chymotrypsin inhibitor of Microcystis aeruginosa NIVA Cya 43. Journal of Natural Products, 68(9), 1324-1327. https://doi.org/10.1021/np050079r

Walls, J. T., Wyatt, K. H., Doll, J. C., Rubenstein, E. M., \& Rober, A. R. (2018). Hot and toxic: Temperature regulates microcystin release from cyanobacteria. Science of the Total Environment, 610-611, 786-795. https://doi.org/10.1016/j.scitotenv.2017.08.149

Watanabe, M. F., Harada, K., Carmichael, W. W., \& Fujiki, H. (1996). Toxic Microcystis.

Welker, M., Maršálek, B., Šejnohová, L., \& von Döhren, H. (2006). Detection and identification of oligopeptides in Microcystis (cyanobacteria) colonies: Toward an understanding of metabolic diversity. Peptides, 27(9), 2090-2103.

https://doi.org/10.1016/j.peptides.2006.03.014 
Williams, D. E., Craig, M., Holmes, C. F. B., \& Andersen, R. J. (1996). Ferintoic acids A and B, new cyclic hexapeptides from the freshwater cyanobacterium Microcystis aeruginosa. Journal of Natural Products, 59(6), 570-575. https://doi.org/10.1021/np9601081

Yadav, S., Sinha, R. P., Tyagi, M. B., \& Kumar, A. (2011). Cyanobacterial secondary metabolites. International Journal of Pharma and Bio Sciences, 2(2), 144-167. https://doi.org/10.1007/978-94-009-0213-8_33

Zhang, Y., Chen, M., Bruner, S. D., \& Ding, Y. (2018). Heterologous Production of Microbial Ribosomally Synthesized and Post-translationally Modified Peptides. 9(August), 1-13. https://doi.org/10.3389/fmicb.2018.01801

Zhang, Y., Li, K., Yang, G., McBride, J. L., Bruner, S. D., \& Ding, Y. (2018). A distributive peptide cyclase processes multiple microviridin core peptides within a single polypeptide substrate. Nature Communications, 9(1). https://doi.org/10.1038/s41467-018-04154-3

Ziemert, N., Ishida, K., Weiz, A., Hertweck, C., \& Dittmann, E. (2010). Exploiting the natural diversity of microviridin gene clusters for discovery of novel tricyclic depsipeptides. Applied and Environmental Microbiology, 76(11), 3568-3574.

https://doi.org/10.1128/AEM.02858-09

Zong, W., Wang, X., Du, Y., Zhang, S., Zhang, Y., \& Teng, Y. (2017). Molecular Mechanism for the Regulation of Microcystin Toxicity to Protein Phosphatase 1 by Glutathione Conjugation Pathway. BioMed Research International, 2017. https://doi.org/10.1155/2017/9676504

Zurawell, R. W., Chen, H., Burke, J. M., \& Prepas, E. E. (2005). Hepatotoxic cyanobacteria: A review of the biological importance of microcystins in freshwater environments. Journal of Toxicology and Environmental Health - Part B: Critical Reviews, 8(1), 1-37. https://doi.org/10.1080/10937400590889412

\section{Supplementary Information}

Table S1: LC-HRMS data for cyanotoxins produced by M. aeruginosa CPCC 299, 300, 632 and 633, and M. flos-aquae CPCC 461

\begin{tabular}{|l|l|l|l|l|l|l|l|l|l|l|l|l|}
\hline Cluster & Cyanotoxin group & Name & {$[\mathbf{M}+\mathbf{H}]^{+}$} & Formula & Error & RT & \multicolumn{4}{|c|}{ Microcystis strain (CPCC) } \\
\cline { 7 - 11 }
\end{tabular}




\begin{tabular}{|c|c|c|c|c|c|c|c|c|c|c|}
\hline 1 & Cyanopeptolin & Uk Сур 904 & $887.4652^{1}$ & $\mathrm{C}_{46} \mathrm{H}_{64} \mathrm{~N}_{8} \mathrm{O}_{11}$ & -1.08 & 4.2 & + & & + & \\
\hline 1 & Cyanopeptolin & Uk Сур 920 & $903.4367^{1}$ & $\mathrm{C}_{43} \mathrm{H}_{65} \mathrm{~N}_{8} \mathrm{O}_{12} \mathrm{Cl}$ & -1.37 & 3.40 & & & + & \\
\hline 1 & Cyanopeptolin & NP BN 920 & $903.4587^{1}$ & $\mathrm{C}_{46} \mathrm{H}_{64} \mathrm{~N}_{8} \mathrm{O}_{12}$ & 0.10 & 3.25 & & & + & \\
\hline 1 & Cyanopeptolin & Uk Сур 932 & $915.4972^{1}$ & $\mathrm{C}_{48} \mathrm{H}_{68} \mathrm{~N}_{8} \mathrm{O}_{11}$ & -0.29 & 4.85 & & & & + \\
\hline 1 & Cyanopeptolin & Uk Cyp 941 & $923.4039^{1}$ & $\mathrm{C}_{45} \mathrm{H}_{65} \mathrm{~N}_{8} \mathrm{O}_{12} \mathrm{Cl}$ & -0.45 & 3.38 & & & + & \\
\hline 1 & Cyanopeptolin & Uk Сур 946 & $929.5116^{1}$ & $\mathrm{C}_{49} \mathrm{H}_{70} \mathrm{~N}_{8} \mathrm{O}_{11}$ & -1.63 & 5.19 & & & & + \\
\hline 1 & Cyanopeptolin & Сyp 954 & $937.4217^{1}$ & $\mathrm{C}_{46} \mathrm{H}_{63} \mathrm{~N}_{8} \mathrm{O}_{12} \mathrm{Cl}$ & -0.43 & 3.51 & & & + & \\
\hline 1 & Cyanopeptolin & Uk Cyp 954 & $937.4441^{1}$ & $\mathrm{C}_{49} \mathrm{H}_{62} \mathrm{~N}_{8} \mathrm{O}_{12}$ & -1.41 & 4.16 & + & & + & \\
\hline 1 & Cyanopeptolin & Uk Сур 945 & 946.4917 & $\mathrm{C}_{49} \mathrm{H}_{67} \mathrm{~N}_{7} \mathrm{O}_{12}$ & -0.36 & 5.35 & & + & & \\
\hline 1 & Cyanopeptolin & Uk Cyp 966 & $949.4821^{1}$ & $\mathrm{C}_{51} \mathrm{H}_{66} \mathrm{~N}_{8} \mathrm{O}_{11}$ & 0.29 & 5.00 & + & & & + \\
\hline 1 & Cyanopeptolin & Uk Сур 968 & $951.4596^{1}$ & $\mathrm{C}_{50} \mathrm{H}_{64} \mathrm{~N}_{8} \mathrm{O}_{12}$ & -1.55 & 3.96 & & & + & + \\
\hline 1 & Cyanopeptolin & Uk Cyp 980 & $963.4964^{1}$ & $\mathrm{C}_{52} \mathrm{H}_{68} \mathrm{~N}_{8} \mathrm{O}_{11}$ & -1.04 & 5.31 & & & & + \\
\hline 1 & Cyanopeptolin & Uk Сур 982 & $965.4525^{1}$ & $\mathrm{C}_{48} \mathrm{H}_{67} \mathrm{~N}_{8} \mathrm{O}_{12} \mathrm{Cl}$ & 1.19 & 4.02 & & & + & \\
\hline 1 & Cyanopeptolin & Uk Cyp 992 & $979.4930^{1}$ & $\mathrm{C}_{52} \mathrm{H}_{68} \mathrm{~N}_{8} \mathrm{O}_{12}$ & 0.73 & 4.79 & & & & + \\
\hline 1 & Cyanopeptolin & Uk Cyp 1010 & $993.4856^{1}$ & $\mathrm{C}_{50} \mathrm{H}_{71} \mathrm{~N}_{8} \mathrm{O}_{12} \mathrm{Cl}$ & 0.08 & 4.97 & & & + & + \\
\hline 1 & Cyanopeptolin & Uk Cyp 999 & 1000.5120 & $\mathrm{C}_{51} \mathrm{H}_{69} \mathrm{~N}_{9} \mathrm{O}_{12}$ & -1.34 & 3.90 & + & & & \\
\hline 2 & Cyanopeptolin & Uk Cyp 900 & 901.5031 & $\mathrm{C}_{44} \mathrm{H}_{68} \mathrm{~N}_{8} \mathrm{O}_{12}$ & 0.17 & 3.17 & & + & & \\
\hline 2 & Cyanopeptolin & Cyp B & 929.5327 & $\mathrm{C}_{46} \mathrm{H}_{72} \mathrm{~N}_{8} \mathrm{O}_{12}$ & -1.66 & 3.69 & & + & & \\
\hline 2 & Cyanopeptolin & Cyp C & 943.5481 & $\mathrm{C}_{47} \mathrm{H}_{74} \mathrm{~N}_{8} \mathrm{O}_{12}$ & -1.90 & 3.70 & & + & & \\
\hline 2 & Cyanopeptolin & Cyp A & 957.5391 & $\mathrm{C}_{46} \mathrm{H}_{72} \mathrm{~N}_{10} \mathrm{O}_{12}$ & -1.35 & 3.75 & & + & & \\
\hline 2 & Cyanopeptolin & Сур 963A & 964.5040 & $\mathrm{C}_{49} \mathrm{H}_{69} \mathrm{~N}_{7} \mathrm{O}_{13}$ & 1.53 & 5.31 & & + & & \\
\hline 2 & Cyanopeptolin & Uk Cyp 970 & 971.5565 & $\mathrm{C}_{47} \mathrm{H}_{74} \mathrm{~N}_{10} \mathrm{O}_{12}$ & 0.46 & 4.14 & & + & & \\
\hline 2 & Cyanopeptolin & Uk Сур 984 & 985.5725 & $\mathrm{C}_{48} \mathrm{H}_{76} \mathrm{~N}_{10} \mathrm{O}_{12}$ & 0.61 & 4.72 & & + & & \\
\hline 3 & Microviridin & Uk Mv 1630 & $816.4052^{2}$ & $\mathrm{C}_{72} \mathrm{H}_{112} \mathrm{~N}_{17} \mathrm{O}_{26}$ & -0.35 & 2.81 & & & + & + \\
\hline 3 & Microviridin & Uk Mv 1651 & $826.9047^{2}$ & $\mathrm{C}_{86} \mathrm{H}_{107} \mathrm{~N}_{16} \mathrm{O}_{18}$ & -0.02 & 2.70 & + & & + & \\
\hline 3 & Microviridin & Uk Mv 1669 & $835.9076^{2}$ & $\mathrm{C}_{80} \mathrm{H}_{113} \mathrm{~N}_{14} \mathrm{O}_{25}$ & 0.36 & 2.79 & + & & & \\
\hline 3 & Microviridin & Uk Mv 1804 & $903.4258^{2}$ & $\mathrm{C}_{88} \mathrm{H}_{114} \mathrm{~N}_{19} \mathrm{O}_{23}$ & 2.00 & 2.80 & + & & + & \\
\hline 3 & Microviridin & Uk Mv 1856 & $922.3999^{2}$ & $\mathrm{C}_{87} \mathrm{H}_{112} \mathrm{~N}_{17} \mathrm{O}_{28}$ & -0.55 & 2.81 & + & & + & \\
\hline 3 & Microviridin & Uk Mv 1850 & $926.4323^{2}$ & $\mathrm{C}_{93} \mathrm{H}_{116} \mathrm{~N}_{19} \mathrm{O}_{22}$ & -2.18 & 2.86 & & & & + \\
\hline 3 & Microviridin & Uk Mv 1858 & $930.4302^{2}$ & $\mathrm{C}_{91} \mathrm{H}_{116} \mathrm{~N}_{19} \mathrm{O}_{24}$ & 1.13 & 2.87 & + & & + & \\
\hline 3 & Microviridin & Uk Mv 1874 & $938.4132^{2}$ & $\mathrm{C}_{88} \mathrm{H}_{116} \mathrm{~N}_{17} \mathrm{O}_{29}$ & -1.75 & 2.89 & & & + & \\
\hline 4 & Microviridin & Uk Mv 1567 & $784.8342^{2}$ & $\mathrm{C}_{80} \mathrm{H}_{91} \mathrm{~N}_{14} \mathrm{O}_{20}$ & 0.31 & 3.21 & + & & & + \\
\hline 4 & Microviridin & Uk Mv 1589 & $800.3928^{2}$ & $\mathrm{C}_{71} \mathrm{H}_{109} \mathrm{~N}_{17} \mathrm{O}_{25}$ & 0.61 & 2.64 & & & + & + \\
\hline 4 & Microviridin & Mv J & $842.8925^{2}$ & $\mathrm{C}_{80} \mathrm{H}_{105} \mathrm{~N}_{19} \mathrm{O}_{22}$ & 1.31 & 2.75 & + & & + & \\
\hline
\end{tabular}




\begin{tabular}{|c|c|c|c|c|c|c|c|c|c|c|c|}
\hline 4 & Microviridin & Uk Mv 1715 & $858.9321^{2}$ & $\mathrm{C}_{88} \mathrm{H}_{115} \mathrm{~N}_{16} \mathrm{O}_{20}$ & 1.36 & 2.90 & + & & & + & \\
\hline 4 & Microviridin & Uk Mv 1822 & $912.4276^{2}$ & $\mathrm{C}_{88} \mathrm{H}_{116} \mathrm{~N}_{19} \mathrm{O}_{24}$ & -1.87 & 2.80 & + & & & + & \\
\hline 4 & Microviridin & $\mathrm{Mv} \mathrm{H}$ & $919.4114^{2}$ & $\mathrm{C}_{89} \mathrm{H}_{114} \mathrm{~N}_{17} \mathrm{O}_{26}$ & -2.11 & 2.87 & + & & & + & + \\
\hline 5 & Microviridin & Uk Mv 1459 & $730.8624^{2}$ & $\mathrm{C}_{82} \mathrm{H}_{91} \mathrm{~N}_{16} \mathrm{O}_{10}$ & -0.15 & 2.44 & + & & & & + \\
\hline 5 & Microviridin & Uk Mv 1612 & $807.4003^{2}$ & $\mathrm{C}_{72} \mathrm{H}_{111} \mathrm{~N}_{17} \mathrm{O}_{25}$ & 0.06 & 2.80 & & & & & + \\
\hline 5 & Microviridin & Uk Mv 1790 & $896.4191^{2}$ & $\mathrm{C}_{80} \mathrm{H}_{116} \mathrm{~N}_{19} \mathrm{O}_{28}$ & 0.06 & 2.64 & + & & & + & \\
\hline 5 & Microviridin & Uk Mv 1826 & $914.4168^{2}$ & $\mathrm{C}_{90} \mathrm{H}_{112} \mathrm{~N}_{19} \mathrm{O}_{23}$ & 0.66 & 2.81 & + & & & + & \\
\hline 6 & Microcystin & {$\left[\mathrm{Asp}^{3}, \mathrm{Dha}^{7}\right]-\mathrm{MC}-\mathrm{LR}$} & 967.5254 & $\mathrm{C}_{47} \mathrm{H}_{70} \mathrm{~N}_{10} \mathrm{O}_{12}$ & -1.69 & 3.29 & + & + & & & \\
\hline 6 & Microcystin & [Asp $\left.{ }^{3}\right]-\mathrm{MC}-\mathrm{LR}$ & 981.5385 & $\mathrm{C}_{48} \mathrm{H}_{72} \mathrm{~N}_{10} \mathrm{O}_{12}$ & -0.71 & 3.40 & + & + & & & \\
\hline 6 & Microcystin & MC-LR & 995.5565 & $\mathrm{C}_{49} \mathrm{H}_{74} \mathrm{~N}_{10} \mathrm{O}_{12}$ & 1.76 & 3.49 & + & + & & & \\
\hline 6 & Microcystin & Uk MC 1010 & 1011.5535 & $\mathrm{C}_{49} \mathrm{H}_{74} \mathrm{~N}_{10} \mathrm{O}_{13}$ & -0.41 & 3.37 & + & + & & & \\
\hline 7 & Microginin & Mg 612 & 613.3225 & $\mathrm{C}_{32} \mathrm{H}_{44} \mathrm{~N}_{4} \mathrm{O}_{8}$ & -1.11 & 2.53 & & & & & + \\
\hline 7 & Microginin & Uk Mg 626 & 627.3374 & $\mathrm{C}_{33} \mathrm{H}_{46} \mathrm{~N}_{4} \mathrm{O}_{8}$ & 0.49 & 2.70 & & & & + & + \\
\hline 7 & Microginin & Uk Mg 628 & 629.3184 & $\mathrm{C}_{32} \mathrm{H}_{44} \mathrm{~N}_{4} \mathrm{O}_{9}$ & 0.11 & 2.31 & & & & & + \\
\hline 7 & Microginin & Uk Mg 630 & 631.2891 & $\mathrm{C}_{32} \mathrm{H}_{43} \mathrm{~N}_{4} \mathrm{O}_{7} \mathrm{Cl}$ & -0.32 & 2.60 & & & & & + \\
\hline 7 & Microginin & Uk Mg 640 & 641.3535 & $\mathrm{C}_{34} \mathrm{H}_{48} \mathrm{~N}_{4} \mathrm{O}_{8}$ & -1.54 & 2.75 & & & & + & + \\
\hline 7 & Microginin & Mg 646 & 647.2826 & $\mathrm{C}_{32} \mathrm{H}_{43} \mathrm{~N}_{4} \mathrm{O}_{8} \mathrm{Cl}$ & -2.22 & 2.56 & & & & & + \\
\hline 7 & Microginin & Uk Mg 660 & 661.2985 & $\mathrm{C}_{33} \mathrm{H}_{45} \mathrm{~N}_{4} \mathrm{O}_{8} \mathrm{Cl}$ & -1.66 & 2.70 & & & & + & + \\
\hline 7 & Microginin & Uk Mg 664 & 665.2503 & $\mathrm{C}_{32} \mathrm{H}_{42} \mathrm{~N}_{4} \mathrm{O}_{7} \mathrm{Cl}_{2}$ & 0.41 & 3.04 & & & & & + \\
\hline 7 & Microginin & Uk Mg 674 & 675.3148 & $\mathrm{C}_{34} \mathrm{H}_{47} \mathrm{~N}_{4} \mathrm{O}_{8} \mathrm{Cl}$ & -1.06 & 2.72 & & & & + & + \\
\hline 7 & Microginin & Mg 680 & 681.2441 & $\mathrm{C}_{32} \mathrm{H}_{42} \mathrm{~N}_{4} \mathrm{O}_{8} \mathrm{Cl}_{2}$ & -1.44 & 2.65 & & & & & + \\
\hline 7 & Microginin & Uk Mg 694 & 695.2596 & $\mathrm{C}_{33} \mathrm{H}_{44} \mathrm{~N}_{4} \mathrm{O}_{8} \mathrm{Cl}_{2}$ & -1.97 & 2.75 & & & & & + \\
\hline 7 & Microginin & Uk Mg 694 & 695.2599 & $\mathrm{C}_{33} \mathrm{H}_{44} \mathrm{~N}_{4} \mathrm{O}_{8} \mathrm{Cl}_{2}$ & $\begin{array}{l}-0.39 \\
\end{array}$ & 2.89 & & & & & + \\
\hline 7 & Microginin & Uk Mg 714 & 715.2053 & $\mathrm{C}_{32} \mathrm{H}_{41} \mathrm{~N}_{4} \mathrm{O}_{8} \mathrm{Cl}_{3}$ & -1.36 & 2.86 & & & & + & + \\
\hline 8 & Aeruginosamide & Uk Ag 560 & 561.2522 & $\mathrm{C}_{31} \mathrm{H}_{36} \mathrm{~N}_{4} \mathrm{O}_{4} \mathrm{~S}$ & $\begin{array}{l}-0.68 \\
\end{array}$ & 2.97 & & & & & + \\
\hline 8 & Aeruginosamide & Uk Ag 562 & 563.2695 & $\mathrm{C}_{31} \mathrm{H}_{38} \mathrm{~N}_{4} \mathrm{O}_{4} \mathrm{~S}$ & -1.86 & 3.09 & & & & & + \\
\hline 8 & Aeruginosamide & $\mathrm{Ag} \mathrm{B}$ & 575.2678 & $\mathrm{C}_{32} \mathrm{H}_{39} \mathrm{~N}_{4} \mathrm{O}_{4} \mathrm{~S}$ & 0.43 & 3.55 & & + & + & & + \\
\hline 8 & Aeruginosamide & Uk Ag 590 & 591.2635 & $\mathrm{C}_{32} \mathrm{H}_{38} \mathrm{~N}_{4} \mathrm{O}_{5} \mathrm{~S}$ & -0.28 & 3.08 & & & & & + \\
\hline 8 & Aeruginosamide & Uk Ag 661 & 660.3217 & $\mathrm{C}_{36} \mathrm{H}_{46} \mathrm{~N}_{5} \mathrm{O}_{5} \mathrm{~S}$ & 0.08 & 3.16 & & & & & + \\
\hline 8 & Aeruginosamide & $\mathrm{Ag} \mathrm{C}$ & 674.3357 & $\mathrm{C}_{37} \mathrm{H}_{47} \mathrm{~N}_{5} \mathrm{O}_{5} \mathrm{~S}$ & 0.79 & 3.85 & & & & & + \\
\hline 8 & Aeruginosamide & Uk Ag 659 & 690.3328 & $\mathrm{C}_{37} \mathrm{H}_{47} \mathrm{~N}_{5} \mathrm{O}_{6} \mathrm{~S}$ & 1.18 & 3.56 & & & & & + \\
\hline 9 & Anabaenopeptin & Ap D & 828.4298 & $\mathrm{C}_{44} \mathrm{H}_{57} \mathrm{~N}_{7} \mathrm{O}_{9}$ & -0.06 & 3.91 & & & & + & + \\
\hline 9 & Anabaenopeptin & Uk Ap 850 & 851.4453 & $\mathrm{C}_{46} \mathrm{H}_{58} \mathrm{~N}_{8} \mathrm{O}_{8}$ & -0.10 & 4.97 & & & & + & + \\
\hline 9 & Anabaenopeptin & Uk Ap 857 & 858.438 & $\mathrm{C}_{45} \mathrm{H}_{59} \mathrm{~N}_{7} \mathrm{O}_{10}$ & -1.82 & 3.44 & & & & + & + \\
\hline
\end{tabular}




\begin{tabular}{|c|c|c|c|c|c|c|c|c|c|c|c|}
\hline 9 & Anabaenopeptin & FA A & 867.4378 & $\mathrm{C}_{46} \mathrm{H}_{58} \mathrm{~N}_{8} \mathrm{O}_{9}$ & -1.44 & 3.93 & & & & + & + \\
\hline 9 & Anabaenopeptin & FA B & 881.4552 & $\mathrm{C}_{47} \mathrm{H}_{60} \mathrm{~N}_{8} \mathrm{O}_{9}$ & 1.13 & 4.18 & & & & + & + \\
\hline 9 & Anabaenopeptin & Uk Ap 882 & 883.4334 & $\mathrm{C}_{46} \mathrm{H}_{58} \mathrm{~N}_{8} \mathrm{O}_{10}$ & -1.66 & 3.46 & & & & + & + \\
\hline 10 & Aeruginosin & Uk Ag 722 & $643.2986^{4}$ & $\mathrm{C}_{32} \mathrm{H}_{43} \mathrm{~N}_{6} \mathrm{O}_{9} \mathrm{SCl}$ & -1.38 & 2.60 & & & & + & \\
\hline 10 & Aeruginosin & Uk Ag 650 & 651.3141 & $\mathrm{C}_{33} \mathrm{H}_{42} \mathrm{~N}_{6} \mathrm{O}_{6}$ & 0.70 & 2.24 & & + & & & \\
\hline 10 & Aeruginosin & Uk Ag 684 & 685.2750 & $\mathrm{C}_{33} \mathrm{H}_{41} \mathrm{~N}_{6} \mathrm{O}_{6} \mathrm{Cl}$ & 0.63 & 2.34 & & + & & & \\
\hline 10 & Aeruginosin & Uk Ag 688 & 689.2947 & $\mathrm{C}_{32} \mathrm{H}_{44} \mathrm{~N}_{6} \mathrm{O}_{9} \mathrm{~S}$ & -1.34 & 2.50 & & & & + & \\
\hline 10 & Aeruginosin & Uk Ag 734 & $717.3002^{1}$ & $\mathrm{C}_{29} \mathrm{H}_{46} \mathrm{~N}_{6} \mathrm{O}_{7} \mathrm{Cl}_{2}$ & 0.98 & 2.44 & & + & & & \\
\hline 11 & Cyanobactin & $\mathrm{Md} \mathrm{A}$ & 583.1901 & $\mathrm{C}_{26} \mathrm{H}_{30} \mathrm{~N}_{8} \mathrm{O}_{4} \mathrm{~S}_{2}$ & 0.85 & 3.15 & & & + & & \\
\hline 11 & Cyanobactin & Uk Md 584 & 585.2058 & $\mathrm{C}_{26} \mathrm{H}_{32} \mathrm{~N}_{8} \mathrm{O}_{4} \mathrm{~S}_{2}$ & -0.03 & 2.96 & + & & + & & \\
\hline 11 & Cyanobactin & Uk Md 586 & 587.2216 & $\mathrm{C}_{26} \mathrm{H}_{34} \mathrm{~N}_{8} \mathrm{O}_{4} \mathrm{~S}_{2}$ & -0.20 & 3.84 & + & & + & & \\
\hline 12 & Cyanobactin & Ac A & 535.2161 & $\mathrm{C}_{24} \mathrm{H}_{34} \mathrm{~N}_{6} \mathrm{O}_{4} \mathrm{~S}_{2}$ & 0.98 & 5.78 & + & + & & & \\
\hline 12 & Cyanobactin & Ac B & 533.2006 & $\mathrm{C}_{24} \mathrm{H}_{32} \mathrm{~N}_{6} \mathrm{O}_{4} \mathrm{~S}_{2}$ & 1.27 & 5.82 & & + & & & \\
\hline 12 & Cyanobactin & Ac C & 517.2235 & $\mathrm{C}_{24} \mathrm{H}_{32} \mathrm{~N}_{6} \mathrm{O}_{5} \mathrm{~S}$ & 1.42 & 5.85 & + & + & & & \\
\hline 12 & Cyanobactin & Uk Ac 518 & 519.2385 & $\mathrm{C}_{24} \mathrm{H}_{34} \mathrm{~N}_{6} \mathrm{O}_{5} \mathrm{~S}$ & -0.89 & 5.77 & & + & & & \\
\hline 13 & Unknown & Uk 1448 & $725.3962^{2}$ & $\mathrm{C}_{73} \mathrm{H}_{106} \mathrm{~N}_{15} \mathrm{O}_{14} \mathrm{~S}$ & 1.02 & 5.38 & + & & & & + \\
\hline 13 & Unknown & Uk 1461 & $731.4159^{2}$ & $\mathrm{C}_{68} \mathrm{H}_{114} \mathrm{~N}_{15} \mathrm{O}_{18} \mathrm{~S}$ & -0.92 & 5.81 & + & & & & + \\
\hline 13 & Unknown & Uk 1462 & $732.4059^{2}$ & $\mathrm{C}_{70} \mathrm{H}_{110} \mathrm{~N}_{16} \mathrm{O}_{16} \mathrm{~S}$ & -2.16 & 5.50 & + & & & & + \\
\hline 13 & Unknown & Uk 1474 & $738.4238^{2}$ & $\mathrm{C}_{69} \mathrm{H}_{116} \mathrm{~N}_{15} \mathrm{O}_{18} \mathrm{~S}$ & -0.75 & 5.81 & + & & & & + \\
\hline 13 & Unknown & Uk 1476 & $739.4133^{2}$ & $\mathrm{C}_{71} \mathrm{H}_{112} \mathrm{~N}_{16} \mathrm{O}_{16} \mathrm{~S}$ & -1.42 & 5.61 & + & & & & + \\
\hline 13 & Unknown & Uk 1488 & $745.4307^{2}$ & $\mathrm{C}_{70} \mathrm{H}_{118} \mathrm{~N}_{15} \mathrm{O}_{18} \mathrm{~S}$ & -2.07 & 5.91 & + & & & & + \\
\hline 13 & Unknown & Uk 1490 & $746.4202^{2}$ & $\mathrm{C}_{72} \mathrm{H}_{114} \mathrm{~N}_{16} \mathrm{O}_{16} \mathrm{~S}$ & -1.69 & 5.56 & + & & & & + \\
\hline 13 & Unknown & Uk 1504 & $753.4296^{2}$ & $\mathrm{C}_{73} \mathrm{H}_{116} \mathrm{~N}_{16} \mathrm{O}_{16} \mathrm{~S}$ & -1.95 & 5.69 & + & & & & + \\
\hline 13 & Unknown & Uk 1508 & $755.4265^{2}$ & $\mathrm{C}_{73} \mathrm{H}_{118} \mathrm{~N}_{15} \mathrm{O}_{15} \mathrm{~S}_{2}$ & 0.67 & 5.41 & + & & & & + \\
\hline 13 & Unknown & Uk 1522 & $762.4341^{2}$ & $\mathrm{C}_{74} \mathrm{H}_{120} \mathrm{~N}_{15} \mathrm{O}_{15} \mathrm{~S}_{2}$ & 0.74 & 5.57 & + & & & & + \\
\hline 14 & Unknown & Uk 676 & $659.3628^{1}$ & $\mathrm{C}_{30} \mathrm{H}_{48} \mathrm{~N}_{10} \mathrm{O}_{8}$ & -0.11 & 5.75 & & + & & + & + \\
\hline 14 & Unknown & Uk 678 & $661.3791^{1}$ & $\mathrm{C}_{30} \mathrm{H}_{50} \mathrm{~N}_{10} \mathrm{O}_{8}$ & 0.12 & 5.94 & & + & & + & + \\
\hline 14 & Unknown & Uk 816 & $799.4315^{1}$ & $\mathrm{C}_{35} \mathrm{H}_{56} \mathrm{~N}_{14} \mathrm{O}_{9}$ & -0.82 & 6.12 & & & & + & + \\
\hline 15 & Unknown & Uk 3314 & $1105.9709^{3}$ & $\mathrm{C}_{188} \mathrm{H}_{247} \mathrm{~N}_{27} \mathrm{O}_{27}$ & 2.34 & 6.05 & + & & & + & + \\
\hline 15 & Unknown & Uk 3316 & $1106.6386^{3}$ & $\mathrm{C}_{188} \mathrm{H}_{249} \mathrm{~N}_{27} \mathrm{O}_{27}$ & -0.05 & 5.84 & & & & + & + \\
\hline 15 & Unknown & Uk 3953 & $1318.7494^{3}$ & $\mathrm{C}_{198} \mathrm{H}_{297} \mathrm{~N}_{41} \mathrm{O}_{44}$ & 0.01 & 6.05 & & & & + & + \\
\hline 16 & Unknown & Uk 612 & 613.3250 & $\mathrm{C}_{32} \mathrm{H}_{44} \mathrm{~N}_{4} \mathrm{O}_{8}$ & 2.96 & 2.95 & & & + & & \\
\hline 16 & Unknown & Uk 692 & 693.2809 & $\mathrm{C}_{32} \mathrm{H}_{44} \mathrm{~N}_{4} \mathrm{O}_{11} \mathrm{~S}$ & 1.34 & 3.03 & & & + & & \\
\hline 16 & Unknown & Uk 794 & 795.2213 & $\mathrm{C}_{34} \mathrm{H}_{42} \mathrm{~N}_{4} \mathrm{O}_{15} \mathrm{~S}_{2}$ & -0.05 & 3.00 & & & + & & \\
\hline
\end{tabular}




\begin{tabular}{|c|c|c|c|c|c|c|c|c|c|c|c|}
\hline 16 & Unknown & Uk 810 & 811.1867 & $\mathrm{C}_{40} \mathrm{H}_{35} \mathrm{~N}_{4} \mathrm{O}_{13} \mathrm{~S}$ & -2.61 & 3.02 & & & + & & \\
\hline 17 & Unknown & Uk 1013 & 1014.6271 & $\mathrm{C}_{56} \mathrm{H}_{83} \mathrm{~N}_{7} \mathrm{O}_{10}$ & -0.27 & 6.04 & & & & + & + \\
\hline 17 & Unknown & Uk 1029 & 1030.6211 & $\mathrm{C}_{56} \mathrm{H}_{83} \mathrm{~N}_{7} \mathrm{O}_{11}$ & 0.22 & 5.91 & & & & + & + \\
\hline 18 & Unknown & Uk 359 & 360.1929 & $\mathrm{C}_{19} \mathrm{H}_{25} \mathrm{~N}_{3} \mathrm{O}_{4}$ & 2.82 & 2.33 & + & & & & \\
\hline 18 & Unknown & Uk 559 & 560.2925 & $\mathrm{C}_{24} \mathrm{H}_{41} \mathrm{~N}_{5} \mathrm{O}_{10}$ & -0.21 & 2.20 & + & & & & \\
\hline 19 & Unknown & Uk 608 & 609.4340 & $\mathrm{C}_{31} \mathrm{H}_{56} \mathrm{~N}_{6} \mathrm{O}_{6}$ & 1.00 & 6.22 & & + & & & \\
\hline 19 & Unknown & Uk 622 & 623.4494 & $\mathrm{C}_{32} \mathrm{H}_{58} \mathrm{~N}_{6} \mathrm{O}_{6}$ & 0.64 & 6.24 & & + & & & \\
\hline 20 & Unknown & Uk 495 & $513.3036^{5}$ & $\mathrm{C}_{23} \mathrm{H}_{40} \mathrm{~N}_{6} \mathrm{O}_{7}$ & 1.02 & 6.25 & & & & + & + \\
\hline 20 & Unknown & Uk 674 & $692.3845^{5}$ & $\mathrm{C}_{25} \mathrm{H}_{46} \mathrm{~N}_{12} \mathrm{O}_{10}$ & -0.80 & 5.49 & + & + & + & + & + \\
\hline 21 & Unknown & Uk 669 & 670.4092 & $\mathrm{C}_{38} \mathrm{H}_{51} \mathrm{~N}_{7} \mathrm{O}_{4}$ & 2.28 & 5.62 & + & & + & & \\
\hline 21 & Unknown & Uk 831 & 832.4614 & $\mathrm{C}_{44} \mathrm{H}_{61} \mathrm{~N}_{7} \mathrm{O}_{9}$ & 1.29 & 5.63 & + & & + & & + \\
\hline 22 & Unknown & Uk 758 & 759.3113 & $\mathrm{C}_{37} \mathrm{H}_{42} \mathrm{~N}_{8} \mathrm{O}_{10}$ & 0.03 & 5.45 & & & & + & + \\
\hline 22 & Unknown & Uk 760 & 761.3274 & $\mathrm{C}_{37} \mathrm{H}_{44} \mathrm{~N}_{8} \mathrm{O}_{10}$ & 2.72 & 5.66 & & + & & + & + \\
\hline 23 & Unknown & Uk 410 & $393.2088^{1}$ & $\mathrm{C}_{19} \mathrm{H}_{30} \mathrm{~N}_{4} \mathrm{O}_{6}$ & -2.36 & 2.32 & & & & + & + \\
\hline 23 & Unknown & Uk 477 & 478.2753 & $\mathrm{C}_{22} \mathrm{H}_{35} \mathrm{~N}_{7} \mathrm{O}_{5}$ & -1.70 & 2.17 & & & & + & + \\
\hline 24 & Unknown & Uk 334 & 335.1596 & $\mathrm{C}_{17} \mathrm{H}_{22} \mathrm{~N}_{2} \mathrm{O}_{5}$ & -1.63 & 2.89 & & + & & + & + \\
\hline 24 & Unknown & Uk 366 & $349.1753^{1}$ & $\mathrm{C}_{17} \mathrm{H}_{26} \mathrm{~N}_{4} \mathrm{O}_{5}$ & -2.71 & 3.55 & & & & + & + \\
\hline 25 & Unknown & Uk 516 & $534.3126^{5}$ & $\mathrm{C}_{23} \mathrm{H}_{40} \mathrm{~N}_{4} \mathrm{O}_{9}$ & -0.82 & 2.28 & & & + & & + \\
\hline 25 & Unknown & Uk 560 & $578.3389^{5}$ & $\mathrm{C}_{25} \mathrm{H}_{44} \mathrm{~N}_{4} \mathrm{O}_{10}$ & -0.16 & 2.30 & & & + & & + \\
\hline 25 & Unknown & Uk 604 & $622.3652^{5}$ & $\mathrm{C}_{27} \mathrm{H}_{48} \mathrm{~N}_{4} \mathrm{O}_{11}$ & -0.70 & 2.32 & & & + & & + \\
\hline 26 & Unknown & Uk 946 & 947.5921 & $\mathrm{C}_{46} \mathrm{H}_{78} \mathrm{~N}_{10} \mathrm{O}_{11}$ & -0.34 & 6.20 & + & + & & & \\
\hline 26 & Unknown & Uk 960 & 961.6081 & $\mathrm{C}_{47} \mathrm{H}_{80} \mathrm{~N}_{10} \mathrm{O}_{11}$ & 0.07 & 6.29 & + & + & & & \\
\hline 26 & Unknown & Uk 974 & 975.6237 & $\mathrm{C}_{48} \mathrm{H}_{82} \mathrm{~N}_{10} \mathrm{O}_{11}$ & 0.05 & 6.32 & & + & & & \\
\hline
\end{tabular}

${ }^{1}\left[\mathrm{M}+\mathrm{H}-\mathrm{H}_{2} \mathrm{O}\right]^{+} ;{ }^{2}[\mathrm{M}+2 \mathrm{H}]^{2+} ;{ }^{3}[\mathrm{M}+3 \mathrm{H}]^{3+} ;{ }^{4}\left[\mathrm{M}+\mathrm{H}-\mathrm{SO}_{3}\right]^{+} ;{ }^{5}\left[\mathrm{M}+\mathrm{NH}_{4}\right]^{+}$

Abbreviations used in the table: Ac; aerucyclamide, Ag; aeruginosamide, Ahp; 3-amino-6hydroxy-2-piperidone, Ap; anabaenopeptin, Cyp; cyanopeptolin, FA; ferintoic acid, Lxx; leucine or isoleucine, MC; microcystin, $\mathrm{Md}$; microcyclamide, $\mathrm{Mg}$; microginin, $\mathrm{Mv}$; microviridin, $\mathrm{NP}$; nostopeptin, Uk; unknown

Table S2: Compiled sequence database for cyanopeptolins previously reported in literature.

\begin{tabular}{|c|c|c|c|c|c|c|c|c|c|c|c|}
\hline $\begin{array}{c}\text { Compoun } \\
\text { d name }\end{array}$ & Formula & $\begin{array}{c}\text { Theoretic } \\
\text { al } \\
{[\mathbf{M}+\mathbf{H}]+}\end{array}$ & $\begin{array}{c}\text { Theoretic } \\
\text { al }[\mathrm{M}+\mathrm{H}- \\
\mathrm{H2O}]+\end{array}$ & Residue 1 & Residue 2 & Residue 3 & Residue 4 & Residue 5 & Residue 6 & Residue 7 & Residue 8 \\
\hline $\begin{array}{c}\text { Tasipeptin } \\
\text { B }\end{array}$ & $\begin{array}{c}\text { C40 H62 } \\
\text { N6 O9 }\end{array}$ & 771.4651 & 753.4545 & L-Val & $\begin{array}{c}\text { NMe-L- } \\
\text { Phe }\end{array}$ & L-Leu & Ahp & L-Leu & L-Thr & & \\
\hline $\begin{array}{l}\text { Micropept } \\
\text { in MZ771 }\end{array}$ & $\begin{array}{c}\text { C38 H61 } \\
\text { N9 O8 }\end{array}$ & 772.4716 & 754.4610 & Ile & NMe-Phe & Ile & OMe-Ahp & Arg & Thr & & \\
\hline $\begin{array}{l}\text { Micropept } \\
\text { in MM836 }\end{array}$ & $\begin{array}{c}\text { C43 H60 } \\
\text { N6 O11 }\end{array}$ & 837.4393 & 819.4287 & Ile & NMe-Phe & Phe & Ahp & Leu & Thr & & \\
\hline
\end{tabular}




\begin{tabular}{|c|c|c|c|c|c|c|c|c|c|c|c|}
\hline $\begin{array}{c}\text { planktope } \\
\text { ptin } \\
\text { BL843 }\end{array}$ & $\begin{array}{c}\text { C41 H61 } \\
\text { N7 O12 }\end{array}$ & 844.4451 & 826.4345 & L-Ile & $\begin{array}{c}\text { NMe- } \\
\text { OMe-L- } \\
\text { Tyr }\end{array}$ & L-Thr & Ahp & L-Leu & L-Thr & L-Gln & \\
\hline $\begin{array}{l}\text { Micropept } \\
\text { in MZ845 }\end{array}$ & $\begin{array}{c}\text { C40 H62 } \\
\text { N9 O11 }\end{array}$ & 846.4720 & 828.4614 & Ile & NMe-Phe & Ile & Ahp & Arg & Thr & & \\
\hline $\begin{array}{l}\text { Micropept } \\
\text { in MM850 }\end{array}$ & $\begin{array}{c}\text { C44 H62 } \\
\text { N6 O11 }\end{array}$ & 851.4549 & 833.4444 & Ile & NMe-Phe & Phe & OMe-Ahp & Leu & Thr & & \\
\hline $\begin{array}{l}\text { Micropept } \\
\text { in MZ859 }\end{array}$ & $\begin{array}{c}\text { C41 H65 } \\
\text { N9 O11 }\end{array}$ & 860.4876 & 842.4771 & Ile & NMe-Phe & Ile & OMe-Ahp & Arg & Thr & & \\
\hline $\begin{array}{c}\text { Tasipeptin } \\
\text { A }\end{array}$ & $\begin{array}{r}\text { C45 H71 } \\
\text { N7 O10 } \\
\end{array}$ & 870.5335 & 852.5230 & L-Val & $\begin{array}{c}\text { NMe-L- } \\
\text { Phe }\end{array}$ & L-Leu & Ahp & L-Leu & L-Thr & L-Val & \\
\hline $\begin{array}{l}\text { Cyanopep } \\
\text { tolin } 880\end{array}$ & $\begin{array}{c}\text { C45 H64 } \\
\text { N6 O12 } \\
\end{array}$ & 881.4655 & 863.4549 & Ile & NMe-Phe & Ile & Ahp & Htyr & Thr & & \\
\hline $\begin{array}{c}\text { micropept } \\
\text { in } 88 \mathrm{~A}\end{array}$ & $\begin{array}{r}\text { C44 H65 } \\
\text { N7 O12 } \\
\end{array}$ & 884.4764 & 866.4658 & L-Ile & $\begin{array}{c}\text { NMe-L- } \\
\text { Phe }\end{array}$ & L-Val & Ahp & HcAla & L-Thr & L-Glu & \\
\hline $\begin{array}{l}\text { Micropept } \\
\text { in } \\
\text { HU895A }\end{array}$ & $\begin{array}{c}\text { C40 H62 } \\
\text { Cl } \\
\text { N9 O12 }\end{array}$ & 896.4279 & 878.4174 & Val & $\begin{array}{c}\text { NMe- } \\
\text { OMe-Cl- } \\
\text { L-Tyr }\end{array}$ & Ile & Ahp & Arg & Thr & & \\
\hline $\begin{array}{c}\text { Micropept } \\
\text { in } \\
\text { HU895B }\end{array}$ & $\begin{array}{c}\text { C40 H62 } \\
\text { Cl } \\
\text { N9 O12 } \\
\end{array}$ & 896.4279 & 878.4174 & Ile & $\begin{array}{l}\text { L-NMe- } \\
\text { Cl-Tyr }\end{array}$ & Ile & Ahp & Arg & Thr & & \\
\hline $\begin{array}{l}\text { Micropept } \\
\text { in HU909 }\end{array}$ & $\begin{array}{c}\text { C41 H64 } \\
\text { Cl } \\
\text { N9 O12 } \\
\end{array}$ & 910.4436 & 892.4330 & Val & $\begin{array}{c}\text { NMe- } \\
\text { OMe-Cl- } \\
\text { L-Tyr } \\
\end{array}$ & Ile & OMe-Ahp & Arg & Thr & & \\
\hline $\begin{array}{c}\text { micropept } \\
\text { in SF909 }\end{array}$ & $\begin{array}{c}\text { C45 H63 } \\
\text { N7 O13 }\end{array}$ & 910.4557 & 892.4451 & L-Ile & $\begin{array}{c}\text { L-NMe- } \\
\text { Tyr }\end{array}$ & L-Leu & Ahp & L-Gln & L-Thr & L-Hpla & \\
\hline $\begin{array}{c}\text { Micropept } \\
\text { in } \\
\text { LH911A }\end{array}$ & $\begin{array}{l}\text { C39 H61 } \\
\text { N9 O14 S }\end{array}$ & 912.4131 & 894.4026 & Val & NMe-Phe & Ile & Ahp & Arg & Thr & & \\
\hline $\begin{array}{l}\text { Micropept } \\
\text { in } \\
\text { LH911B }\end{array}$ & $\begin{array}{l}\text { C39 H61 } \\
\text { N9 O14 S }\end{array}$ & 912.4131 & 894.4026 & Ile & NMe-Phe & Val & Ahp & Arg & Thr & & \\
\hline $\begin{array}{c}\text { Micropept } \\
\text { in } \\
\text { LH911C }\end{array}$ & $\begin{array}{l}\text { C41 H65 } \\
\text { N7 O14 S }\end{array}$ & 912.4383 & 894.4277 & Ile & NMe-Phe & Ile & Ahp & NMe-Lys & Thr & & \\
\hline $\begin{array}{l}\text { Micropept } \\
\text { in MM916 }\end{array}$ & $\begin{array}{l}\mathrm{C} 43 \mathrm{H} 60 \\
\mathrm{~N} 6 \mathrm{O} 14 \mathrm{~S} \\
\end{array}$ & 917.3961 & 899.3855 & Ile & NMe-Phe & Phe & Ahp & Leu & Thr & & \\
\hline $\begin{array}{c}\text { Somamide } \\
\text { B }\end{array}$ & $\begin{array}{c}\text { C46 H62 } \\
\text { N8 O12 } \\
\end{array}$ & 919.4560 & 901.4454 & Val & NMe-Tyr & Phe & Ahp & $\mathrm{Abu}$ & Thr & Gln & \\
\hline $\begin{array}{c}\text { Nostopept } \\
\text { in BN9 }\end{array}$ & $\begin{array}{c}\text { C46 H64 } \\
\text { N8 O12 } \\
\end{array}$ & 921.4716 & 903.4611 & L-Val & $\begin{array}{c}\text { NMe-L- } \\
\text { Tyr }\end{array}$ & L-Phe & Ahp & L-Leu & L-Thr & L-Gln & \\
\hline $\begin{array}{l}\text { Micropept } \\
\text { in LH920 }\end{array}$ & $\begin{array}{c}\text { C46 H64 } \\
\text { N8 O12 } \\
\end{array}$ & 921.4716 & 903.4611 & Val & NMe-Tyr & Phe & Ahp & Gln & Thr & Gly & \\
\hline $\begin{array}{l}\text { Cyanopep } \\
\text { tolin } 920\end{array}$ & $\begin{array}{c}\text { C47 H68 } \\
\text { N8 O11 }\end{array}$ & 921.5080 & 903.4975 & Val & NMe-Phe & Phe & Ahp & Lys & Thr & Leu & \\
\hline $\begin{array}{c}\text { cyanopept } \\
\text { olin S }\end{array}$ & $\begin{array}{l}\text { C40 H63 } \\
\text { N9 O14 S } \\
\end{array}$ & 926.4288 & 908.4182 & L-Ile & $\begin{array}{c}\text { NMe-L- } \\
\text { Phe }\end{array}$ & L-Ile & Ahp & L-Arg & L-Thr & & \\
\hline $\begin{array}{l}\text { Micropept } \\
\text { in MZ925 }\end{array}$ & $\begin{array}{c}\mathrm{C} 40 \mathrm{H} 63 \\
\mathrm{~N} 9 \mathrm{O} 14 \mathrm{~S} \\
\end{array}$ & 926.4288 & 908.4182 & Ile & NMe-Phe & Ile & Ahp & Arg & Thr & & \\
\hline $\begin{array}{l}\text { Micropept } \\
\text { in LH925 }\end{array}$ & $\begin{array}{l}\text { C42 H67 } \\
\text { N7 O14 S }\end{array}$ & 926.4539 & 908.4434 & Ile & NMe-Phe & Ile & Ahp & $\begin{array}{c}\text { N,NdiMe- } \\
\text { Lys }\end{array}$ & Thr & & \\
\hline $\begin{array}{c}\text { nostopepti } \\
\text { n B }\end{array}$ & $\begin{array}{c}\mathrm{C} 46 \mathrm{H} 70 \\
\mathrm{~N} 8 \mathrm{O} 12 \\
\end{array}$ & 927.5186 & 909.5080 & L-Ile & $\begin{array}{c}\text { NMe-L- } \\
\text { Tyr }\end{array}$ & L-Ile & Ahp & L-Leu & Hmp & L-Gln & \\
\hline $\begin{array}{c}\text { nostopepti } \\
\text { n C }\end{array}$ & $\begin{array}{c}\mathrm{C} 46 \mathrm{H} 70 \\
\mathrm{~N} 8 \mathrm{O} 12 \\
\end{array}$ & 927.5186 & 909.5080 & Ile & $\begin{array}{c}\text { NMe-L- } \\
\text { Tyr }\end{array}$ & Ile & Ahp & Leu & Hmp & Gln & \\
\hline $\begin{array}{l}\text { Micropept } \\
\text { in KB928 }\end{array}$ & $\begin{array}{l}\mathrm{C} 44 \mathrm{H} 68 \\
\mathrm{~N} 10 \mathrm{O} 12 \\
\end{array}$ & 929.5091 & 911.4985 & Ile & NMe-Phe & Val & Ahp & Arg & Thr & Asp & \\
\hline $\begin{array}{c}\text { Cyanopep } \\
\text { tolin B }\end{array}$ & $\begin{array}{c}\text { C46 H72 } \\
\text { N8 O12 } \\
\end{array}$ & 929.5342 & 911.5237 & Val & NMe-Phe & Leu & Ahp & Lys & Thr & Asp & \\
\hline $\begin{array}{l}\text { Cyanopep } \\
\text { tolin } 930\end{array}$ & $\begin{array}{c}\text { C45 H70 } \\
\text { N8 O13 } \\
\end{array}$ & 931.5135 & 913.5029 & Val & NMe-Tyr & Leu & Ahp & Lys & Thr & Glu & \\
\hline $\begin{array}{l}\text { Micropept } \\
\text { in MM932 }\end{array}$ & $\begin{array}{l}\text { C43 H60 } \\
\text { N6 O15 S }\end{array}$ & 933.3910 & 915.3804 & Ile & NMe-Tyr & Phe & Ahp & Leu & Thr & & \\
\hline $\begin{array}{c}\text { Micropept } \\
\text { in } \\
\text { MZ939A }\end{array}$ & $\begin{array}{c}\text { C41 H65 } \\
\text { N9 O14 S }\end{array}$ & 940.4444 & 922.4339 & Ile & NMe-Phe & Ile & OMe-Ahp & Arg & Thr & & \\
\hline
\end{tabular}




\begin{tabular}{|c|c|c|c|c|c|c|c|c|c|c|c|}
\hline $\begin{array}{l}\text { Micropept } \\
\text { in } \\
\text { MZ939B }\end{array}$ & $\begin{array}{c}\text { C41 H65 } \\
\text { N9 O14 S }\end{array}$ & 940.4444 & 922.4339 & Ile & NMe-Phe & Ile & OMe-Ahp & Arg & Thr & & \\
\hline $\begin{array}{c}\text { Cyanopep } \\
\text { tolin C }\end{array}$ & $\begin{array}{r}\text { C47 H74 } \\
\text { N8 O12 } \\
\end{array}$ & 943.5499 & 925.5393 & Val & NMe-Phe & Leu & Ahp & NMe-Lys & Thr & Asp & \\
\hline $\begin{array}{l}\text { micropept } \\
\text { in SD944 }\end{array}$ & $\begin{array}{c}\mathrm{C} 46 \mathrm{H} 72 \\
\mathrm{~N} 8 \mathrm{O} 13 \\
\end{array}$ & 945.5292 & 927.5186 & L-Val & $\begin{array}{c}\text { NMe-L- } \\
\text { Tyr }\end{array}$ & L-Ile & Ahp & L-Lys & L-Thr & L-Asp & \\
\hline $\begin{array}{c}\text { Scyptolin } \\
\text { A }\end{array}$ & $\begin{array}{c}\mathrm{C} 45 \mathrm{H} 69 \\
\mathrm{Cl} \\
\mathrm{N} 8 \mathrm{O} 14 \\
\end{array}$ & 946.5006 & 928.4900 & Val & $\begin{array}{l}\text { L-NMe- } \\
\text { Cl-Tyr }\end{array}$ & Thr & Ahp & Leu & Thr & Thr & Ala \\
\hline $\begin{array}{l}\text { Micropept } \\
\text { in KT } 946\end{array}$ & $\begin{array}{c}\text { C44 H62 } \\
\text { N6 O15 S }\end{array}$ & 947.4067 & 929.3961 & Ile & NMe-Phe & Ile & Ahp & Htyr & Thr & & \\
\hline $\begin{array}{c}\text { Anabaeno } \\
\text { peptilide } \\
90 \mathrm{~A}\end{array}$ & $\begin{array}{c}\text { C46 H64 } \\
\text { N8 O14 }\end{array}$ & 953.4615 & 935.4509 & Ile & $\begin{array}{c}\text { NMe- } \\
\text { OMe-Tyr }\end{array}$ & Thr & Ahp & Htyr & Thr & Gln & \\
\hline $\begin{array}{l}\text { Cyanopep } \\
\text { tolin } 954\end{array}$ & $\begin{array}{c}\mathrm{C} 46 \mathrm{H} 63 \\
\mathrm{Cl} \\
\mathrm{N} 8 \mathrm{O} 12 \\
\end{array}$ & 955.4327 & 937.4221 & L-Val & $\begin{array}{l}\text { NMe-Cl- } \\
\text { L-Tyr }\end{array}$ & L-Phe & Ahp & L-Leu & L-Thr & L-Gln & \\
\hline $\begin{array}{c}\text { nostopepti } \\
\text { n A }\end{array}$ & $\begin{array}{c}\mathrm{C} 48 \mathrm{H} 74 \\
\mathrm{~N} 8 \mathrm{O} 12\end{array}$ & 955.5499 & 937.5393 & L-Ile & $\begin{array}{c}\text { NMe-L- } \\
\text { Tyr }\end{array}$ & L-Ile & Ahp & L-Leu & Hmp & L-Gln & \\
\hline $\begin{array}{c}\text { nostopepti } \\
\text { n D }\end{array}$ & $\begin{array}{c}\text { C48 H74 } \\
\text { N8 O12 }\end{array}$ & 955.5499 & 937.5393 & L-Ile & $\begin{array}{c}\text { NMe-L- } \\
\text { Tyr }\end{array}$ & L-Ile & Ahp & L-Leu & Hmp & L-Gln & \\
\hline $\begin{array}{c}\text { nostopepti } \\
n \text { E }\end{array}$ & $\begin{array}{r}\text { C47 H73 } \\
\text { N9 O12 } \\
\end{array}$ & 956.5451 & 938.5346 & L-Ile & $\begin{array}{c}\text { NMe-L- } \\
\text { Tyr }\end{array}$ & L-Leu & Ahp & L-Leu & Hmp & L-Gln & \\
\hline $\begin{array}{c}\text { Cyanopep } \\
\text { tolin A }\end{array}$ & $\begin{array}{l}\text { C46 H72 } \\
\text { N10 O12 }\end{array}$ & 957.5404 & 939.5298 & Val & NMe-Phe & Leu & Ahp & Arg & Thr & Asp & \\
\hline $\begin{array}{l}\text { Micropept } \\
\text { in KB956 }\end{array}$ & $\begin{array}{l}\mathrm{C} 46 \mathrm{H} 72 \\
\mathrm{~N} 10 \mathrm{O} 12\end{array}$ & 957.5404 & 939.5298 & Ile & NMe-Phe & Val & OMe-Ahp & Arg & Thr & OMe-Asp & \\
\hline $\begin{array}{c}\text { Cyanopep } \\
\text { tolin D }\end{array}$ & $\begin{array}{c}\text { C48 H76 } \\
\text { N8 O12 }\end{array}$ & 957.5655 & 939.5550 & Val & NMe-Phe & Leu & Ahp & $\begin{array}{l}\text { N,NdiMe- } \\
\text { Lys }\end{array}$ & Thr & Asp & \\
\hline $\begin{array}{l}\text { Cyanopep } \\
\text { tolin } 958\end{array}$ & $\begin{array}{l}\mathrm{C} 45 \mathrm{H} 70 \\
\mathrm{~N} 10 \mathrm{O} 13 \\
\end{array}$ & 959.5197 & 941.5091 & Val & NMe-Tyr & Leu & Ahp & Arg & Thr & Glu & \\
\hline $\begin{array}{l}\text { micropept } \\
\text { in B }\end{array}$ & $\begin{array}{c}\text { C47 H74 } \\
\text { N8 O13 }\end{array}$ & 959.5448 & 941.5342 & L-Val & $\begin{array}{c}\text { NMe-L- } \\
\text { Tyr }\end{array}$ & L-Leu & Ahp & L-Arg & L-Thr & L-Glu & \\
\hline $\begin{array}{l}\text { Cyanopep } \\
\text { tolin } 960\end{array}$ & $\begin{array}{c}\text { C45 H64 } \\
\text { N6 O15 S }\end{array}$ & 961.4223 & 943.4117 & Ile & NMe-Phe & Ile & Ahp & Htyr & Thr & & \\
\hline $\begin{array}{l}\text { Micropept } \\
\text { in HH } 960\end{array}$ & $\begin{array}{c}\text { C49 H68 } \\
\text { N8 O12 } \\
\end{array}$ & 961.5029 & 943.4924 & Val & NMe-Phe & Leu & Apo & Leu & Thr & Asn & \\
\hline $\begin{array}{l}\text { micropept } \\
\text { in } 90\end{array}$ & $\begin{array}{l}\text { C42 H59 } \\
\text { N9 O15 S }\end{array}$ & 962.3924 & 944.3818 & Val & NMe-Tyr & L-Phe & Ahp & Arg & L-Thr & & \\
\hline $\begin{array}{l}\text { cyanopept } \\
\text { olin 963A }\end{array}$ & $\begin{array}{r}\text { C49 H69 } \\
\text { N7 O13 }\end{array}$ & 964.5026 & 946.4920 & L-Val & $\begin{array}{l}\text { NMe-L- } \\
\text { Phe }\end{array}$ & L-Leu & Ahp & L-Tyr & L-Thr & L-Asp & \\
\hline $\begin{array}{l}\text { micropept } \\
\text { in E1964 }\end{array}$ & $\begin{array}{l}\text { C46 H64 } \\
\text { N10 O13 }\end{array}$ & 965.4727 & 947.4621 & L-Ile & $\begin{array}{c}\text { NMe-L- } \\
\text { Tyr }\end{array}$ & L-Phe & Ahp & L-Arg & L-Thr & L-Asp & \\
\hline $\begin{array}{c}\text { Somamide } \\
\text { A }\end{array}$ & $\begin{array}{c}\text { C48 H67 } \\
\text { N7 O12 S }\end{array}$ & 966.4641 & 948.4536 & Val & NMe-Tyr & Phe & Ahp & Abu & Thr & $\operatorname{Met}(\mathrm{O})$ & \\
\hline $\begin{array}{l}\text { Micropept } \\
\text { in T20 }\end{array}$ & $\begin{array}{c}\text { C46 H59 } \\
\text { N6 O15 P }\end{array}$ & 967.3849 & 949.3743 & L-Ile & $\begin{array}{c}\text { N-Me-L- } \\
\text { Tyr }\end{array}$ & L-Phe & L-Ahp & L-Phe & L-Thr & $\begin{array}{l}\text { D-Gac- } \\
\text { Phos }\end{array}$ & \\
\hline $\begin{array}{c}\text { nostopepti } \\
\text { n I }\end{array}$ & $\begin{array}{r}\text { C48 H75 } \\
\text { N9 O12 }\end{array}$ & 970.5608 & 952.5502 & L-Ile & $\begin{array}{c}\text { NMe- } \\
\text { OMe-L- } \\
\text { Tyr } \\
\end{array}$ & L-Leu & Ahp & L-Leu & Hmp & L-Gln & \\
\hline $\begin{array}{c}\text { nostopepti } \\
\text { n F }\end{array}$ & $\begin{array}{r}\text { C48 H75 } \\
\text { N9 O12 } \\
\end{array}$ & 970.5608 & 952.5502 & L-Ile & $\begin{array}{c}\text { NMe-L- } \\
\text { Tyr }\end{array}$ & L-Leu & OMe-Ahp & L-Leu & Hmp & L-Gln & \\
\hline $\begin{array}{l}\text { Micropept } \\
\text { in } \\
\text { KB970A } \\
\end{array}$ & $\begin{array}{l}\text { C47 H74 } \\
\text { N10 O12 }\end{array}$ & 971.5560 & 953.5455 & Ile & NMe-Phe & Val & Ahp & Arg & Thr & OMe-Asp & \\
\hline $\begin{array}{c}\text { Micropept } \\
\text { in } \\
\text { KB970B }\end{array}$ & $\begin{array}{l}\text { C47 H74 } \\
\text { N10 O12 }\end{array}$ & 971.5560 & 953.5455 & Ile & NMe-Phe & Val & OMe-Ahp & Arg & Thr & Asp & \\
\hline $\begin{array}{c}\text { Micropept } \\
\text { in } \\
\text { KB970C } \\
\end{array}$ & $\begin{array}{l}\text { C47 H74 } \\
\text { N10 O12 }\end{array}$ & 971.5560 & 953.5455 & Val & NMe-Phe & Val & OMe-Ahp & Arg & Thr & OMe-Asp & \\
\hline $\begin{array}{c}\text { Anabaeno } \\
\text { peptilide } \\
90 \mathrm{~B} \\
\end{array}$ & $\begin{array}{c}\text { C45 H61 } \\
\text { Cl } \\
\text { N8 O14 } \\
\end{array}$ & 973.4069 & 955.3963 & Ile & $\begin{array}{l}\text { L-NMe- } \\
\text { Cl-Tyr }\end{array}$ & Thr & Ahp & Htyr & Thr & Gln & \\
\hline $\begin{array}{l}\text { Cyanopep } \\
\text { tolin } 972\end{array}$ & $\begin{array}{l}\text { C46 H72 } \\
\text { N10 O13 }\end{array}$ & 973.5353 & 955.5247 & Val & NMe-Tyr & Leu & Ahp & Arg & Thr & Asp & \\
\hline
\end{tabular}




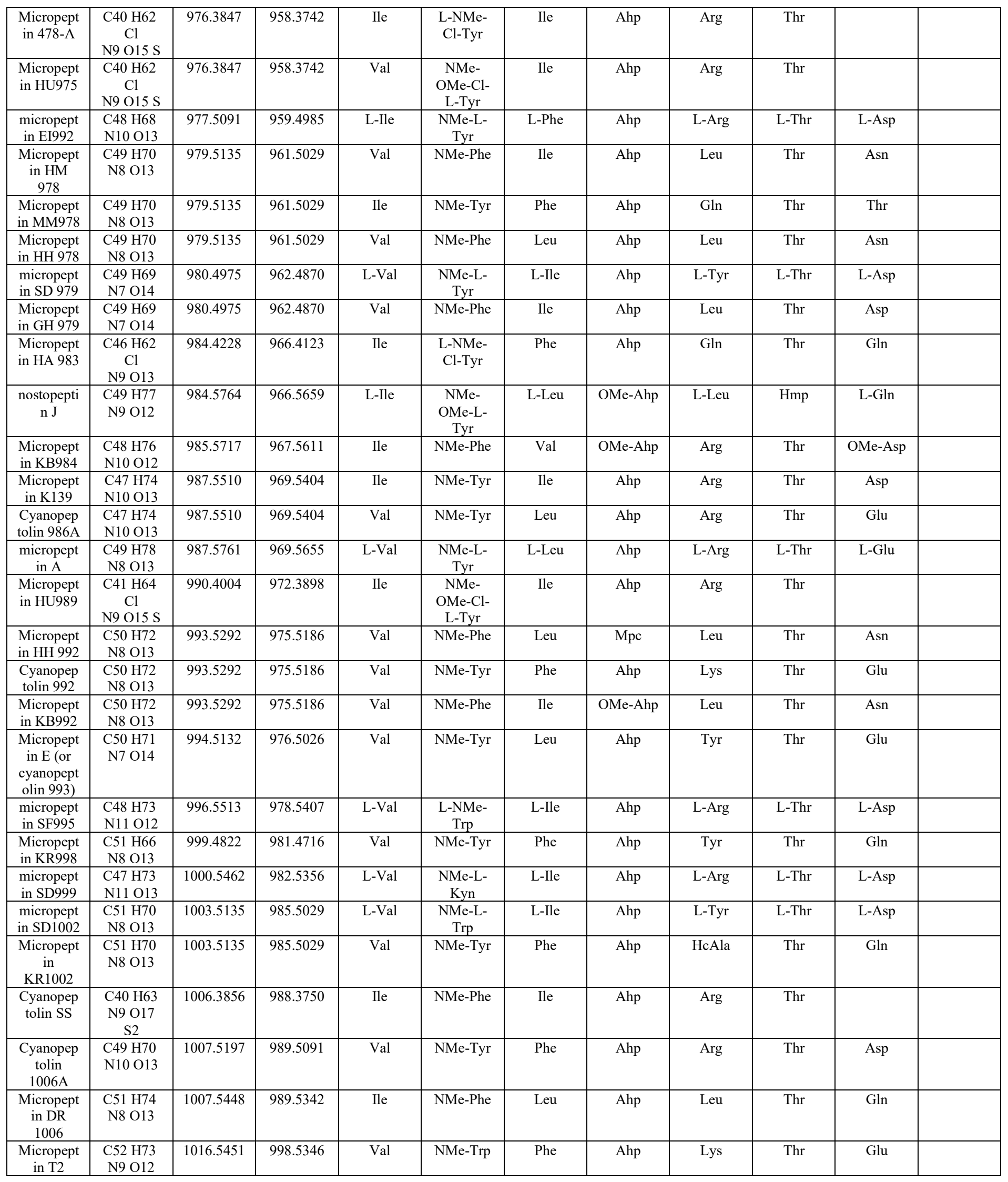




\begin{tabular}{|c|c|c|c|c|c|c|c|c|c|c|c|}
\hline $\begin{array}{c}\text { Micropept } \\
\text { in } \\
\text { MZ1019 }\end{array}$ & $\begin{array}{c}\text { C41 H65 } \\
\text { N9 O17 } \\
\text { S2 }\end{array}$ & 1020.4013 & 1002.3907 & Ile & NMe-Phe & Ile & OMe-Ahp & Arg & Thr & & \\
\hline $\begin{array}{l}\text { Cyanopep } \\
\text { tolin } 1020\end{array}$ & $\begin{array}{l}\mathrm{C} 50 \mathrm{H} 72 \\
\mathrm{~N} 10 \mathrm{O} 13 \\
\end{array}$ & 1021.5353 & 1003.5247 & Val & NMe-Tyr & Phe & Ahp & Arg & Thr & Glu & \\
\hline $\begin{array}{c}\text { Cyanopep } \\
\text { tolin } \\
1020 \mathrm{~B}\end{array}$ & $\begin{array}{c}\mathrm{C} 52 \mathrm{H} 76 \\
\mathrm{~N} 8 \mathrm{O} 13\end{array}$ & 1021.5605 & 1003.5499 & Val & NMe-Tyr & Phe & Ahp & Lys & Thr & Glu & \\
\hline $\begin{array}{c}\text { Micropept } \\
\text { in } \\
\text { HU1021 }\end{array}$ & $\begin{array}{c}\text { C40 H63 } \\
\text { N9 O18 S } \\
2 \\
\end{array}$ & 1022.3805 & 1004.3700 & Ile & $\begin{array}{c}\text { NMe-L- } \\
\text { Tyr }\end{array}$ & Ile & Ahp & Arg & Thr & & \\
\hline $\begin{array}{c}\text { Micropept } \\
\text { in } \\
\text { LH1021 }\end{array}$ & $\begin{array}{r}\text { C50 H71 } \\
\text { N9 O14 }\end{array}$ & 1022.5193 & 1004.5088 & Val & NMe-Tyr & Phe & Ahp & Gln & Thr & Thr & Gly \\
\hline $\begin{array}{l}\text { Micropept } \\
\text { in F (or } \\
\text { cyanopept } \\
\text { olin 1021) }\end{array}$ & $\begin{array}{r}\mathrm{C} 52 \mathrm{H} 75 \\
\mathrm{~N} 7 \mathrm{O} 14\end{array}$ & 1022.5445 & 1004.5339 & Val & NMe-Tyr & Leu & Ahp & Tyr & Thr & Glu & \\
\hline $\begin{array}{c}\text { Aeruginop } \\
\text { eptin } \\
917 \mathrm{~S}-\mathrm{C}\end{array}$ & $\begin{array}{r}\text { C51 H74 } \\
\text { N8 O14 }\end{array}$ & 1023.5397 & 1005.5292 & Ile & NMe-Tyr & Leu & Ahp & Leu & Thr & Gln & \\
\hline $\begin{array}{l}\text { Micropept } \\
\text { in C }\end{array}$ & $\begin{array}{r}\mathrm{C} 53 \mathrm{H} 69 \\
\mathrm{~N} 7 \mathrm{O} 14 \\
\end{array}$ & 1028.4975 & 1010.4870 & Val & NMe-Tyr & Phe & Ahp & Tyr & Thr & Glu & \\
\hline $\begin{array}{c}\text { Micropept } \\
\text { in } \\
\text { KR1030 }\end{array}$ & $\begin{array}{r}\mathrm{C} 53 \mathrm{H} 74 \\
\mathrm{~N} 8 \mathrm{O} 13\end{array}$ & 1031.5448 & 1013.5342 & Val & NMe-Tyr & Phe & Ahp & HcAla & Thr & Gln & \\
\hline $\begin{array}{c}\text { micropept } \\
\text { in } 88 \mathrm{~N}\end{array}$ & $\begin{array}{r}\mathrm{C} 54 \mathrm{H} 78 \\
\mathrm{~N} 8 \mathrm{O} 14 \\
\end{array}$ & 1031.5812 & 1013.5706 & L-Ile & $\begin{array}{c}\text { NMe-L- } \\
\text { Phe }\end{array}$ & L-Val & Ahp & L-Tyr & L-Thr & L-Glu & L-Leu \\
\hline $\begin{array}{l}\text { Oscillapep } \\
\text { tilide 97-B }\end{array}$ & $\begin{array}{r}\mathrm{C} 52 \mathrm{H} 73 \\
\mathrm{~N} 9 \mathrm{O} 13 \\
\end{array}$ & 1032.5401 & 1014.5295 & Ile & NMe-Tyr & Phe & Ahp & Leu & Thr & Gln & Pro \\
\hline $\begin{array}{c}\text { Micropept } \\
\text { in } \\
\text { HU1041 }\end{array}$ & $\begin{array}{c}\text { C39 H60 } \\
\mathrm{Cl} \\
\text { N9 O18 S } \\
2 \\
\end{array}$ & 1042.3259 & 1024.3153 & Val & $\begin{array}{l}\text { L-NMe- } \\
\text { Cl-Tyr }\end{array}$ & Ile & Ahp & Arg & Thr & & \\
\hline $\begin{array}{c}\text { Micropept } \\
\text { in } \\
\text { KT1042 }\end{array}$ & $\begin{array}{r}\text { C53 H70 } \\
\text { N8 O14 }\end{array}$ & 1043.5084 & 1025.4979 & Val & NMe-Phe & Ile & Ahp & Tyr & Thr & Gln & \\
\hline $\begin{array}{c}\text { Aeruginop } \\
\text { eptin } \\
228 \mathrm{~A} \\
\end{array}$ & $\begin{array}{r}\text { C52 H68 } \\
\text { N8 O15 }\end{array}$ & 1045.4877 & 1027.4771 & Ile & NMe-Phe & Thr & Ahp & Tyr & Thr & Gln & \\
\hline $\begin{array}{c}\text { micropept } \\
\text { in } 103\end{array}$ & $\begin{array}{l}\text { C52 H72 } \\
\text { N10 O13 } \\
\end{array}$ & 1045.5353 & 1027.5247 & L-Val & $\begin{array}{c}\text { NMe-L- } \\
\text { Trp }\end{array}$ & L-Phe & Ahp & L-Gln & L-Thr & L-Thr & Gly \\
\hline $\begin{array}{c}\text { Oscillapep } \\
\text { tilide 97- } \\
\text { A }\end{array}$ & $\begin{array}{r}\text { C53 H75 } \\
\text { N9 O13 }\end{array}$ & 1046.5557 & 1028.5451 & Ile & $\begin{array}{c}\text { NMe- } \\
\text { OMe-Tyr }\end{array}$ & Phe & Ahp & Leu & Thr & Gln & Pro \\
\hline $\begin{array}{c}\text { Micropept } \\
\text { in } \\
\text { KB1046 }\end{array}$ & $\begin{array}{r}\mathrm{C} 53 \mathrm{H} 74 \\
\mathrm{~N} 8 \mathrm{O} 14\end{array}$ & 1047.5397 & 1029.5292 & Ile & NMe-Phe & Val & Ahp & HcAla & Thr & Gln & \\
\hline $\begin{array}{c}\text { Aeruginop } \\
\text { eptin } \\
228 \mathrm{~B}\end{array}$ & $\begin{array}{r}\mathrm{C} 52 \mathrm{H} 72 \\
\mathrm{~N} 8 \mathrm{O} 15\end{array}$ & 1049.5190 & 1031.5084 & Ile & NMe-Phe & Thr & Ahp & HcAla & Thr & Gln & \\
\hline $\begin{array}{c}\text { Micropept } \\
\text { in } \\
\text { KB1048 }\end{array}$ & $\begin{array}{c}\mathrm{C} 49 \mathrm{H} 77 \\
\mathrm{Cl} \\
\mathrm{N} 10 \mathrm{O} 13 \\
\end{array}$ & 1049.5433 & 1031.5327 & Ile & $\begin{array}{l}\text { L-NMe- } \\
\text { Cl-Tyr }\end{array}$ & Ile & OMe-Ahp & Arg & Thr & OMe-Asp & \\
\hline $\begin{array}{l}\text { Micropept } \\
\text { in } \\
\text { LH1048 }\end{array}$ & $\begin{array}{l}\text { C52 H76 } \\
\text { N10 O13 }\end{array}$ & 1049.5666 & 1031.5560 & Val & NMe-Tyr & Phe & Ahp & Arg & Thr & Glu & \\
\hline $\begin{array}{l}\text { Cyanopep } \\
\text { tolin } 1048\end{array}$ & $\begin{array}{l}\text { C52 H76 } \\
\text { N10 O13 } \\
\end{array}$ & 1049.5666 & 1031.5560 & Val & NMe-Tyr & Phe & Ahp & Arg & Thr & Glu & \\
\hline $\begin{array}{c}\text { Anabaeno } \\
\text { peptilide } \\
\text { 202A }\end{array}$ & $\begin{array}{r}\text { C51 H71 } \\
\text { N9 O15 }\end{array}$ & 1050.5142 & 1032.5037 & Ile & $\begin{array}{c}\text { NMe- } \\
\text { OMe-Tyr }\end{array}$ & Thr & Ahp & Htyr & Thr & Gln & Pro \\
\hline $\begin{array}{l}\text { Micropept } \\
\text { in T1 }\end{array}$ & $\begin{array}{r}\mathrm{C} 55 \mathrm{H} 70 \\
\mathrm{~N} 8 \mathrm{O} 13 \\
\end{array}$ & 1051.5135 & 1033.5029 & Val & NMe-Trp & Phe & Ahp & Tyr & Thr & Glu & \\
\hline $\begin{array}{c}\text { Symplosta } \\
\text { tin } 2\end{array}$ & $\begin{array}{l}\text { C52 H74 } \\
\text { N8 O13 S }\end{array}$ & 1051.5169 & 1033.5063 & Val & NMe-Tyr & Phe & Ahp & Abu & Thr & $\operatorname{Met}(\mathrm{O})$ & Ile \\
\hline $\begin{array}{c}\text { Symploca } \\
\text { mide A }\end{array}$ & $\begin{array}{c}\text { C46 H71 } \\
\text { Br } \\
\text { N10 O13 } \\
\end{array}$ & 1053.4438 & 1035.4332 & L-Val & $\begin{array}{c}\text { NMe,OM } \\
\text { e-L-Br- } \\
\text { Tyr }\end{array}$ & L-Ile & Ahp & L-Cit & L-Thr & L-Gln & But \\
\hline
\end{tabular}




\begin{tabular}{|c|c|c|c|c|c|c|c|c|c|c|c|}
\hline $\begin{array}{c}\text { Micropept } \\
\text { in 478-B }\end{array}$ & $\begin{array}{c}\text { C40 H62 } \\
\text { Cl } \\
\text { N9 O18 S } \\
2\end{array}$ & 1056.3416 & 1038.3310 & Ile & $\begin{array}{c}\text { L-NMe- } \\
\text { Cl-Tyr }\end{array}$ & Ile & Ahp & Arg & Thr & & \\
\hline $\begin{array}{l}\text { Micropept } \\
\text { in D }\end{array}$ & $\begin{array}{r}\text { C55 H73 } \\
\text { N7 O14 } \\
\end{array}$ & 1056.5288 & 1038.5183 & Val & NMe-Tyr & Phe & Ahp & Tyr & Thr & Glu & \\
\hline $\begin{array}{c}\text { Micropept } \\
\text { in DR } \\
1056\end{array}$ & $\begin{array}{r}\text { C54 H72 } \\
\text { N8 O14 }\end{array}$ & 1057.5241 & 1039.5135 & Ile & NMe-Phe & Leu & Ahp & Tyr & Thr & Gln & \\
\hline $\begin{array}{c}\text { Microcyst } \\
\text { ilide A }\end{array}$ & $\begin{array}{r}\text { C54 H72 } \\
\text { N8 O15 } \\
\end{array}$ & 1057.5241 & 1039.5135 & Ile & NMe-Tyr & Leu & Ahp & Tyr & Thr & Gln & \\
\hline $\begin{array}{c}\text { Micropept } \\
\text { in DR } \\
1060\end{array}$ & $\begin{array}{r}\text { C54 H76 } \\
\text { N8 O14 }\end{array}$ & 1061.5554 & 1043.5448 & Ile & NMe-Phe & Leu & Ahp & HcAla & Thr & Gln & \\
\hline $\begin{array}{c}\text { planktope } \\
\text { ptin } \\
\text { BL1061 }\end{array}$ & $\begin{array}{r}\text { C50 H79 } \\
\text { N9 O16 }\end{array}$ & 1062.5718 & 1044.5612 & L-Ile & $\begin{array}{c}\text { NMe- } \\
\text { OMe-L- } \\
\text { Tyr } \\
\end{array}$ & L-Thr & Ahp & L-Leu & L-Thr & L-Gln & L-Leu \\
\hline $\begin{array}{c}\text { oscillapep } \\
\text { tin C }\end{array}$ & $\begin{array}{r}\text { C55 H79 } \\
\text { N7 O14 } \\
\end{array}$ & 1062.5758 & 1044.5652 & L-allo-Ile & $\begin{array}{c}\text { NMe-L- } \\
\text { Phe }\end{array}$ & L-Ile & OMe-Ahp & HcAla & L-Thr & D-Htyr & \\
\hline $\begin{array}{c}\text { micropept } \\
\text { in } 88 \mathrm{E}\end{array}$ & $\begin{array}{r}\mathrm{C} 54 \mathrm{H} 78 \\
\mathrm{~N} 8 \mathrm{O} 14 \\
\end{array}$ & 1063.5710 & 1045.5605 & L-Ile & $\begin{array}{l}\text { NMe-L- } \\
\text { Phe }\end{array}$ & L-Val & Ahp & L-Leu & L-Thr & L-Glu & L-Phe \\
\hline $\begin{array}{c}\text { Micropept } \\
\text { in } \\
\text { LH1062 }\end{array}$ & $\begin{array}{l}\text { C53 H78 } \\
\text { N10 O13 }\end{array}$ & 1063.5823 & 1045.5717 & Val & NMe-Tyr & Phe & Ahp & Arg & Thr & OMe-Glu & \\
\hline $\begin{array}{c}\text { Micropept } \\
\text { in } \\
\text { HU1069 }\end{array}$ & $\begin{array}{c}\text { C41 H64 } \\
\text { Cl } \\
\text { N9 O18 S } \\
2 \\
\end{array}$ & 1070.3572 & 1052.3466 & Ile & $\begin{array}{c}\text { NMe- } \\
\text { OMe-Cl- } \\
\text { L-Tyr }\end{array}$ & Ile & Ahp & Arg & Thr & & \\
\hline $\begin{array}{c}\text { Anabaeno } \\
\text { peptilide } \\
202 \mathrm{~B}\end{array}$ & $\begin{array}{c}\text { C50 H68 } \\
\text { Cl } \\
\text { N9 O15 } \\
\end{array}$ & 1070.4596 & 1052.4491 & Ile & $\begin{array}{c}\text { L-NMe- } \\
\text { Cl-Tyr }\end{array}$ & Thr & Ahp & Htyr & Thr & Gln & Pro \\
\hline $\begin{array}{c}\text { Aeruginop } \\
\text { eptin } \\
917 \mathrm{~S}-\mathrm{A} \\
\end{array}$ & $\begin{array}{r}\text { C54 H72 } \\
\text { N8 O15 }\end{array}$ & 1073.5190 & 1055.5084 & Ile & NMe-Tyr & Leu & Ahp & Tyr & Thr & Gln & \\
\hline $\begin{array}{c}\text { Hofmanno } \\
\text { lin }\end{array}$ & $\begin{array}{r}\text { C54 H71 } \\
\text { N7 O16 } \\
\end{array}$ & 1074.5030 & 1056.4924 & Val & NMe-Tyr & OMe-Tyr & Ahp & Tyr & Thr & Glu & \\
\hline $\begin{array}{c}\text { Aeruginop } \\
\text { eptin } \\
917 \mathrm{~S}-\mathrm{B}\end{array}$ & $\begin{array}{r}\text { C54 H76 } \\
\text { N8 O15 }\end{array}$ & 1077.5503 & 1059.5397 & Ile & NMe-Tyr & Leu & Ahp & HcAla & Thr & Gln & \\
\hline $\begin{array}{c}\text { Micropept } \\
\text { in } 88 \mathrm{~B} \\
\end{array}$ & $\begin{array}{r}\text { C53 H74 } \\
\text { N8 O16 } \\
\end{array}$ & 1079.5296 & 1061.5190 & L-Ile & $\begin{array}{c}\text { NMe-L- } \\
\text { Phe }\end{array}$ & L-Val & Ahp & L-Glu & L-Thr & L-Glu & L-Phe \\
\hline $\begin{array}{c}\text { micropept } \\
\text { in } 88 \mathrm{Y}\end{array}$ & $\begin{array}{r}\text { C55 H72 } \\
\text { N8 O15 } \\
\end{array}$ & 1085.5190 & 1067.5084 & L-Ile & $\begin{array}{c}\text { NMe-L- } \\
\text { Phe }\end{array}$ & L-Val & Ahp & L-Tyr & L-Thr & L-Glu & L-Tyr \\
\hline $\begin{array}{c}\text { oscillapep } \\
\text { tin F }\end{array}$ & $\begin{array}{l}\text { C51 H76 } \\
\text { N8 O16 S } \\
\end{array}$ & 1089.5173 & 1071.5067 & L-allo-Ile & $\begin{array}{c}\text { NMe-L- } \\
\text { Phe }\end{array}$ & L-Ile & Ahp & L-Lys & L-Thr & D-Htyr & \\
\hline $\begin{array}{l}\text { Cyanopep } \\
\text { tolin } 975\end{array}$ & $\begin{array}{c}\text { C47 H66 } \\
\text { N10 O18 } \\
\text { S } \\
\end{array}$ & 1091.4350 & 1073.4244 & Leu & NMe-Tyr & Phe & Ahp & Arg & Thr & Asp & \\
\hline $\begin{array}{c}\text { Micropept } \\
\text { in } 1106\end{array}$ & $\begin{array}{l}\text { C54 H79 } \\
\text { N11 O14 } \\
\end{array}$ & 1106.5881 & 1088.5775 & Ile & NMe-Phe & Val & Ahp & Arg & Thr & Glu & Tyr \\
\hline $\begin{array}{c}\text { Oscillapep } \\
\operatorname{tin} \mathrm{G}\end{array}$ & $\begin{array}{r}\text { C53 H77 } \\
\text { N9 O17 } \\
\end{array}$ & 1112.5510 & 1094.5405 & Ile & NMe-Tyr & Thr & Ahp & Leu & Thr & Gln & Hty \\
\hline $\begin{array}{c}\text { micropept } \\
\text { in } 88 \mathrm{C} \\
\end{array}$ & $\begin{array}{r}\text { C57 H76 } \\
\text { N8 O15 } \\
\end{array}$ & 1113.5503 & 1095.5397 & L-Ile & $\begin{array}{c}\text { NMe-L- } \\
\text { Phe }\end{array}$ & L-Val & Ahp & L-Tyr & L-Thr & L-Glu & L-Phe \\
\hline $\begin{array}{c}\text { Nostocycl } \\
\text { in }\end{array}$ & $\begin{array}{r}\text { C56 H76 } \\
\text { N8 O16 } \\
\end{array}$ & 1117.5452 & 1099.5346 & Val & NMe-Tyr & Phe & Ahp & Hse & Thr & Hse & Ile \\
\hline $\begin{array}{c}\text { micropept } \\
\text { in } 88 \mathrm{D}\end{array}$ & $\begin{array}{r}\text { C57 H80 } \\
\text { N8 O15 } \\
\end{array}$ & 1117.5816 & 1099.5710 & L-Ile & $\begin{array}{c}\text { NMe-L- } \\
\text { Phe }\end{array}$ & L-Val & Ahp & HcAla & L-Thr & L-Glu & L-Phe \\
\hline $\begin{array}{c}\text { Micropept } \\
\text { in } 1120\end{array}$ & $\begin{array}{l}\text { C55 H81 } \\
\text { N11 O14 } \\
\end{array}$ & 1120.6037 & 1102.5932 & Ile & NMe-Phe & Val & Ahp & Arg & Thr & OMe-Glu & Tyr \\
\hline $\begin{array}{c}\text { Scyptolin } \\
\text { B }\end{array}$ & $\begin{array}{c}\mathrm{C} 52 \mathrm{H} 80 \\
\mathrm{Cl} \\
\mathrm{N} 9 \mathrm{O} 16 \\
\end{array}$ & 1122.5484 & 1104.5379 & Val & $\begin{array}{l}\text { L-NMe- } \\
\text { Cl-Tyr }\end{array}$ & Thr & Ahp & Leu & Thr & Thr & Ala (x2) \\
\hline $\begin{array}{c}\text { planktope } \\
\text { ptin } \\
\text { BL1125 }\end{array}$ & $\begin{array}{r}\text { C54 H79 } \\
\text { N9 O17 }\end{array}$ & 1126.5667 & 1108.5561 & L-Ile & $\begin{array}{c}\mathrm{NMe}, \mathrm{OM} \\
\text { e-L-Tyr }\end{array}$ & L-Thr & Ahp & L-Leu & L-Thr & L-Gln & L-Htyr \\
\hline $\begin{array}{c}\text { micropept } \\
\text { in } 88 \mathrm{~F}\end{array}$ & $\begin{array}{r}\text { C58 H78 } \\
\text { N8 O15 } \\
\end{array}$ & 1127.5659 & 1109.5554 & L-Ile & $\begin{array}{c}\text { NMe-L- } \\
\text { Phe }\end{array}$ & L-Val & Ahp & L-Tyr & L-Thr & $\begin{array}{c}\text { OMe-L- } \\
\text { Glu }\end{array}$ & L-Phe \\
\hline
\end{tabular}




\begin{tabular}{|c|c|c|c|c|c|c|c|c|c|c|c|}
\hline $\begin{array}{c}\text { oscillapep } \\
\text { tin D }\end{array}$ & $\begin{array}{l}\text { C54 H77 } \\
\text { N7 O17 S }\end{array}$ & 1128.5169 & 1110.5064 & L-allo-Ile & $\begin{array}{l}\text { NMe-L- } \\
\text { Phe }\end{array}$ & L-Ile & Ahp & HcAla & L-Thr & D-Htyr & \\
\hline $\begin{array}{l}\text { oscillapep } \\
\text { tin E }\end{array}$ & $\begin{array}{l}\text { C55 H75 } \\
\text { N7 O17 S }\end{array}$ & 1138.5013 & 1120.4907 & L-allo-Ile & $\begin{array}{c}\text { NMe-L- } \\
\text { Phe }\end{array}$ & L-Ile & Ahp & L-Htyr & L-Thr & D-Htyr & \\
\hline $\begin{array}{c}\text { Lyngbyast } \\
\text { atin } 4\end{array}$ & $\begin{array}{l}\text { C53 H68 } \\
\text { N8 O18 S }\end{array}$ & 1139.4403 & 1121.4297 & L-Val & NMe-Tyr & Phe & L-Ahp & Abu & L-Thr & L-Htyr & L-Ala \\
\hline $\begin{array}{l}\text { Aeruginop } \\
\text { eptin 95A }\end{array}$ & $\begin{array}{r}\text { C56 H75 } \\
\text { N9 O17 } \\
\end{array}$ & 1146.5354 & 1128.5248 & Ile & NMe-Phe & Thr & Ahp & Tyr & Thr & Gln & Thr \\
\hline $\begin{array}{l}\text { Oscillapep } \\
\text { tin J }\end{array}$ & $\begin{array}{c}\mathrm{C} 47 \mathrm{H} 68 \\
\mathrm{~N} 10 \mathrm{O} 18 \\
\mathrm{~S} \\
\end{array}$ & 1149.4557 & 1131.4452 & Ile & NMe-Tyr & Thr & Ahp & Arg & Thr & Tyr & \\
\hline $\begin{array}{l}\text { Aeruginop } \\
\text { eptin 95B }\end{array}$ & $\begin{array}{r}\text { C56 H79 } \\
\text { N9 O17 } \\
\end{array}$ & 1150.5667 & 1132.5561 & Ile & NMe-Phe & Thr & Ahp & HcAla & Thr & Gln & Thr \\
\hline $\begin{array}{c}\text { oscillapep } \\
\text { tin A }\end{array}$ & $\begin{array}{l}\text { C56 H77 } \\
\text { N7 O18 S }\end{array}$ & 1168.5119 & 1150.5013 & L-allo-Ile & $\begin{array}{c}\text { NMe- } \\
\text { OMe-L- } \\
\text { Tyr }\end{array}$ & L-Ile & Ahp & L-Htyr & L-Thr & L-Htyr & \\
\hline $\begin{array}{c}\text { Oscillapep } \\
\text { tin }\end{array}$ & $\begin{array}{l}\text { C56 H77 } \\
\text { N7 O18 S }\end{array}$ & 1168.5119 & 1150.5013 & Ile & $\begin{array}{c}\text { NMe- } \\
\text { OMe-Tyr }\end{array}$ & Ile & Ahp & Tyr & Thr & Tyr & \\
\hline $\begin{array}{l}\text { oscillapep } \\
\text { tin B }\end{array}$ & $\begin{array}{l}\text { C57 H79 } \\
\text { N7 O18 S }\end{array}$ & 1182.5275 & 1164.5169 & L-allo-Ile & $\begin{array}{c}\text { NMe- } \\
\text { OMe-L- } \\
\text { Tyr }\end{array}$ & L-Ile & Ahp & $\begin{array}{c}\text { L-7-Me- } \\
\text { Htyr }\end{array}$ & L-Thr & L-Htyr & \\
\hline $\begin{array}{c}\text { largamide } \\
\text { E }\end{array}$ & $\begin{array}{c}\mathrm{C} 56 \mathrm{H} 82 \\
\mathrm{Cl} \\
\mathrm{N} 9 \mathrm{O} 17\end{array}$ & 1188.5590 & 1170.5484 & L-Val & $\begin{array}{l}\text { L-NMe- } \\
\text { Cl-Tyr }\end{array}$ & L-Thr & L-Ahp & L-Leu & L-Thr & L-Val & L-Ala \\
\hline $\begin{array}{c}\text { largamide } \\
\text { D }\end{array}$ & $\begin{array}{c}\mathrm{C} 56 \mathrm{H} 82 \\
\mathrm{Br} \\
\mathrm{N} 9 \mathrm{O} 17\end{array}$ & 1232.5085 & 1214.4979 & L-Val & $\begin{array}{l}\text { L-NMe- } \\
\text { Br-Tyr }\end{array}$ & L-Thr & L-Ahp & L-Leu & L-Thr & L-Val & L-Ala \\
\hline $\begin{array}{c}\text { largamide } \\
\text { F }\end{array}$ & $\begin{array}{c}\mathrm{C} 59 \mathrm{H} 80 \\
\mathrm{Br} \\
\mathrm{N} 9 \mathrm{O} 18 \\
\end{array}$ & 1282.4878 & 1264.4772 & L-Val & $\begin{array}{l}\text { L-NMe- } \\
\text { Br-Tyr }\end{array}$ & L-Thr & L-Ahp & L-Tyr & L-Thr & L-Val & L-Ala \\
\hline$\underset{G}{\text { largamide }}$ & $\begin{array}{c}\mathrm{C} 60 \mathrm{H} 82 \\
\mathrm{Br} \\
\mathrm{N} 9 \mathrm{O} 18 \\
\end{array}$ & 1296.5034 & 1278.4928 & L-Val & $\begin{array}{l}\text { L-NMe- } \\
\text { Br-Tyr }\end{array}$ & L-Thr & L-Ahp & L-Htyr & L-Thr & L-Val & L-Ala \\
\hline
\end{tabular}

Table S3: Compiled sequence database for microginins previously reported in literature.

\begin{tabular}{|c|c|c|c|c|c|c|c|c|}
\hline Compound name & $\begin{array}{l}\text { Molecular } \\
\text { formula }\end{array}$ & $\begin{array}{c}\text { Theoretical } \\
m / z \\
{[\mathbf{M}+\mathbf{H}]^{+}}\end{array}$ & Residue 1 & Residue 2 & Residue 3 & Residue 4 & Residue 5 & Residue 6 \\
\hline MG FR1 & C36 H57 N5 O9 & 728.4229 & Ahda & Ala & NMe-Leu & Tyr & Tyr & \\
\hline
\end{tabular}




\begin{tabular}{|c|c|c|c|c|c|c|c|}
\hline MG FR2 & C39 H59 N5 O9 & 742.4386 & $N M e-A h d a$ & Ala & MLeu & Tyr & Tyr \\
\hline MG FR3 & C37 H53 N5 O10 & 728.3865 & Ahda & Thr & Pro & Tyr & Tyr \\
\hline MG FR4 & C38 H55 N5 O10 & 742.4022 & NMe-Ahda & Thr & Pro & Tyr & Tyr \\
\hline MG FR5 & C33 H55 N5 O9 & 666.4073 & Ahda & Val & Pro & Tyr & Tyr \\
\hline MGFR6 & C39 H57 N5 O9 & 740.4229 & Me Ahda & Val & Pro & Tyr & Tyr \\
\hline MG FR7 & C38 H57 N5 O10 & 744.4178 & Ahda & Ser & Me Leu & Tyr & Tyr \\
\hline MG FR8 & C41 H61 N5 O9 & 768.4542 & Me Ahda & Pro & Me Leu & Tye & Tyr \\
\hline MG FR9 & C39 H54 N6 O9 & 751.4025 & Me Ahda & Thr & Pro & Tyr & Trp \\
\hline MG FR10 & C40 H56 N6 O8 & 749.4232 & Ahda & Val & Pro & Tyr & Trp \\
\hline MG FR12 & C42 H65 N5 O9 & 784.4855 & Me Ahda & Val & Me Leu & Me Tyr & Tyr \\
\hline MG FR13 & C41 H63 N5 O9 & 770.4699 & Me Ahda & Val & Ile/Leu & Me Tyr & Tyr \\
\hline MG & C37 H55 N5 O9 & 714.4073 & Ahda & Ala & Val & Me Tyr & Tyr \\
\hline MG T1 & $\begin{array}{l}\text { C36 H50 Cl N5 } \\
\text { O9 }\end{array}$ & 732.3370 & Cl-Ahda & Ala & Pro & Tyr & Tyr \\
\hline MG T2 & C36 H51 N5 O9 & 698.3760 & Ahda & Ala & Pro & Tyr & Tyr \\
\hline MG 51-A & C49 H68 N6 O11 & 917.5019 & Ahda & Tyr & Me Val & Me Tyr & Pro \\
\hline MG 51-B & C50 H70 N6 O11 & 931.5175 & Me Ahda & Tyr & Me Val & Me Tyr & Pro \\
\hline MG 91-A & $\begin{array}{l}\text { C28 H51 Cl N4 } \\
\text { O6 }\end{array}$ & 575.3570 & Cl Ahda & Ile & Me Leu & Pro & N/A \\
\hline MG 91-B & $\begin{array}{l}\mathrm{C} 28 \mathrm{H} 50 \mathrm{Cl} 2 \mathrm{~N} 4 \\
\mathrm{O} 6\end{array}$ & 609.3180 & Cl2 Ahda & Ile & Me Leu & Pro & N/A \\
\hline MG 91-C & C37 H61 N5 O8 & 704.4593 & Ahda & Ile & Me Leu & Pro & Tyr \\
\hline MG 91-D & $\begin{array}{l}\text { C37 H60 Cl N5 } \\
\text { O8 }\end{array}$ & 738.4203 & Cl Ahda & Ile & Me Leu & Pro & Tyr \\
\hline MG 91-E & $\begin{array}{l}\text { C37 H60 Cl2 N5 } \\
\text { O8 }\end{array}$ & 773.3892 & Cl2 Ahda & Ile & Me Leu & Pro & Tyr \\
\hline MG 99-A & $\begin{array}{l}\mathrm{C} 40 \mathrm{H} 58 \mathrm{Cl} \mathrm{N} 5 \\
\mathrm{O} 8\end{array}$ & 772.4047 & Cl Ahda & Tyr & Leu & Me Tyr & Pro \\
\hline MG 99-B & $\begin{array}{l}\text { C40 H57 Cl2 N5 } \\
\text { O8 }\end{array}$ & 806.3657 & Cl2 Ahda & Tyr & Leu & Me Tyr & Pro \\
\hline
\end{tabular}




\begin{tabular}{|c|c|c|c|c|c|c|c|c|}
\hline MG 299-A & $\begin{array}{l}\text { C45 H67 Cl N6 } \\
\text { O10 }\end{array}$ & 887.4680 & Cl Ahda & Val & Me Val & Me Tyr & Pro & Tyr \\
\hline MG 299-B & $\begin{array}{l}\text { C45 H66 Cl2 N6 } \\
\text { O10 }\end{array}$ & 921.4290 & C12 Ahda & Val & Me Val & Me Tyr & Pro & Tyr \\
\hline MG 299-C & C45 H68 N6 O10 & 853.5070 & Ahda & Val & Me Val & Me Tyr & Pro & Tyr \\
\hline MG $299 \mathrm{D}$ & $\begin{array}{l}\text { C36 H57 Cl2 N5 } \\
\text { O8 }\end{array}$ & 758.3657 & Cl2 Ahda & Val & Me Val & Me Tyr & Pro & \\
\hline MG 478 & C41 H63 N5 O9 & 770.4699 & $N M e-A h d a$ & Val & Me Val & Me Tyr & Tyr & \\
\hline MG 511 & C25 H41 N3 O6 S & 512.2789 & Ahda & Ala & Met & Tyr & N/A & \\
\hline MG 527 & $\mathrm{C} 25 \mathrm{H} 41 \mathrm{~N} 3 \mathrm{O} 7 \mathrm{~S}$ & 528.2738 & Ahda & Met & Tyr & Tyr & $\mathrm{N} / \mathrm{A}$ & \\
\hline MG 527 Methyl Ester & C26 H43 N3 O7 S & 542.2894 & Me Ahda & Met & Tyr & Tyr & N/A & \\
\hline MG 535 & C27H42N4O7 & 535.3000 & Ahda & Ala & Pro & Tyr & $\mathrm{N} / \mathrm{A}$ & \\
\hline MG 551 & $\mathrm{C} 27 \mathrm{H} 42 \mathrm{~N} 4 \mathrm{O} 8$ & 551.1000 & Ahda & Ser & Pro & Tyr & $\mathrm{N} / \mathrm{A}$ & \\
\hline MG 565A & C29H48N4O7 & 565.0000 & Ahda & Ala & Me Leu & Tyr & $\mathrm{N} / \mathrm{A}$ & \\
\hline MG 565B & $\mathrm{C} 29 \mathrm{H} 48 \mathrm{~N} 4 \mathrm{O} 7$ & 565.2000 & Ahda & Thr & Pro & Tyr & N/A & \\
\hline MG 568 & $\mathrm{C} 27 \mathrm{H} 41 \mathrm{~N} 4 \mathrm{O} 7 \mathrm{Cl}$ & 569.1000 & Cl Ahda & Ala & Pro & Tyr & N/A & \\
\hline MG 576 & $\mathrm{C} 26 \mathrm{H} 53 \mathrm{~N} 4 \mathrm{O} 6 \mathrm{Cl}$ & 577.2000 & Cl Ahda & Ile & Me Lxx & Val & $\mathrm{N} / \mathrm{A}$ & \\
\hline MG 577 & $\mathrm{C} 26 \mathrm{H} 53 \mathrm{~N} 4 \mathrm{O} 6 \mathrm{Cl}$ & 638.2300 & Cl Ahda & Ile & Me Lxx & Val & N/A & \\
\hline MG 579A & $\mathrm{C} 30 \mathrm{H} 50 \mathrm{~N} 4 \mathrm{O} 7$ & 578.9000 & Me Ahda & Ala & Me Lxx & Tyr & N/A & \\
\hline MG 579B & C29H46N4O8 & 579.2000 & Me Ahda & Thr & Pro & Tyr & $\mathrm{N} / \mathrm{A}$ & \\
\hline MG 579C & $\mathrm{C} 29 \mathrm{H} 46 \mathrm{~N} 4 \mathrm{O} 8$ & 579.2000 & Ahda & Ala & $\begin{array}{l}\text { Me Tyr/Me } \\
\text { Htyr }\end{array}$ & Tyr & N/A & \\
\hline MG 581 & C29H48N4O8 & 581.2000 & Ahda & Ser & Me Lxx & Tyr & $\mathrm{N} / \mathrm{A}$ & \\
\hline M 591B & C31H50N4O7 & 591.2000 & Ahda & Tyr & Me Lxx & Pro & $\mathrm{N} / \mathrm{A}$ & \\
\hline MG 598 & $\mathrm{C} 28 \mathrm{H} 43 \mathrm{~N} 4 \mathrm{O} 8 \mathrm{Cl}$ & 599.2000 & Cl Ahda & Thr & Pro & Tyr & N/A & \\
\hline MG 607A & $\mathrm{C} 32 \mathrm{H} 54 \mathrm{~N} 4 \mathrm{O} 7$ & 607.4000 & Me Ahda & Val & Leu/Ile & $\mathrm{MeTyr} / \mathrm{Htyr}$ & N/A & \\
\hline MG 607B & $\mathrm{C} 32 \mathrm{H} 54 \mathrm{~N} 4 \mathrm{O} 7$ & 607.4000 & Ahda & Val & $\begin{array}{l}\text { MeLeu/Me } \\
\text { Ile }\end{array}$ & $\mathrm{MeTyr} / \mathrm{Htyr}$ & N/A & \\
\hline MG 612 & C32 H44 N4 O8 & 613.3232 & Ahoa & Gly & Tyr & Tyr & Pro & \\
\hline MG 621A & $\mathrm{C} 33 \mathrm{H} 56 \mathrm{~N} 4 \mathrm{O} 7$ & 621.3000 & Me Ahda & Val & $\begin{array}{l}\mathrm{Me} \mathrm{Leu} / \mathrm{Me} \\
\text { Ile }\end{array}$ & $\mathrm{MeTyr} / \mathrm{Htyr}$ & $\mathrm{N} / \mathrm{A}$ & \\
\hline
\end{tabular}




\begin{tabular}{|c|c|c|c|c|c|c|c|}
\hline MG 621B & $\mathrm{C} 33 \mathrm{H} 56 \mathrm{~N} 4 \mathrm{O} 7$ & 621.4000 & Me Ahda & Val & $\begin{array}{l}\text { Me Leu/Me } \\
\text { Ile }\end{array}$ & Tyr & N/A \\
\hline MG 646 & $\begin{array}{l}\text { C32 H43 Cl N4 } \\
\text { O8 }\end{array}$ & 647.2842 & Cl Ahoa & Gly & Tyr & Tyr & Pro \\
\hline MG 650 & C33H55N5O8 & 650.0000 & Ahda & Ala & Val & Leu/Ile & Tyr \\
\hline MG 674 & C34 H50 N4 O8 S & 675.3422 & Me Ahda & Met & Tyr & Tyr & $\mathrm{N} / \mathrm{A}$ \\
\hline MG 680 & $\begin{array}{l}\mathrm{C} 32 \mathrm{H} 42 \mathrm{Cl} 2 \mathrm{~N} 4 \\
\mathrm{O} 8\end{array}$ & 681.2452 & C12 Ahoa & Gly & Tyr & Tyr & Pro \\
\hline MG 688 & C35 H52 N4 O8 S & 689.3579 & Ahoa & Met & Tyr & Tyr & N/A \\
\hline MG 690 & C34 H50 N4 O9 S & 691.3371 & Me Ahoa & Met & Tyr & Tyr & N/A \\
\hline MG 704 & C35 H52 N4 O9 S & 704.3450 & Ahda & Val & Me Met & Tyr & Me Tyr \\
\hline MG 711 & C39 H59 N5 O6 & 694.4538 & An & Val & Me Leu & Tyr & Tyr \\
\hline MG 713 & C37 H55 N5 O9 & 714.4073 & & & & & \\
\hline MG 715 & $\begin{array}{l}\mathrm{C} 38 \mathrm{H} 50 \mathrm{Cl} \mathrm{O} 7 \\
\mathrm{~N} 5\end{array}$ & 724.3472 & ClAn & Val & Pro & Tyr & Tyr \\
\hline MG 756 & C38 H58 N5 O9 & 729.4307 & Ahda & Val & Leu & HTy & Tyr \\
\hline MG 757 & C39 H59 N5 O10 & 746.4335 & Ahda & Thr & Val & Tyr & Tyr \\
\hline MG 764 & C40 H56 N6 O9 & 765.4182 & Me Ahda & Thr & Pro & Tyr & Trp \\
\hline MG 767 & C41 H61 N5 O9 & 768.4542 & & & & & \\
\hline MG 767B & C41 H61 N5 O9 & 768.4542 & & & & & \\
\hline MG 770 & C39 H60 N5 O9 & 743.4464 & Me Ahda & Val & Leu & HTy & Tyr \\
\hline MG AL584 & $\begin{array}{l}\mathrm{C} 28 \mathrm{H} 45 \mathrm{Cl} \mathrm{N} 4 \\
\mathrm{O} 7\end{array}$ & 585.3050 & Cl Ahda & Gly & Val & Tyr & N/A \\
\hline MG KR604 & C32 H52 N4 O7 & 605.3909 & Me Ahda & Tyr & NMeLeu & Pro & N/A \\
\hline MG KR638 & $\begin{array}{l}\mathrm{C} 32 \mathrm{H} 51 \mathrm{Cl} \mathrm{N} 4 \\
\mathrm{O} 7\end{array}$ & 639.3519 & $\begin{array}{l}\text { Cl- } N \mathrm{Me} \\
\text { Ahda }\end{array}$ & Tyr & NMeLeu & Pro & N/A \\
\hline MG KR767 & C41 H61 N5 O9 & 768.4542 & Me Ahda & Tyr & NMeLeu & Pro & Tyr \\
\hline MG KR781 & C42 H62 N5 O9 & 781.4520 & NMe Ahda & & & & \\
\hline MG KR787 & $\begin{array}{l}\text { C40 H58 Cl N5 } \\
\text { O9 }\end{array}$ & 788.3996 & Cl Ahda & & & & \\
\hline MG KR801 & $\begin{array}{l}\text { C41 H61 Cl N5 } \\
\text { O9 }\end{array}$ & 803.4231 & $\begin{array}{l}\text { Cl- } N \mathrm{Me}- \\
\text { Ahda }\end{array}$ & Tyr & Me Leu & Pro & Tyr \\
\hline
\end{tabular}




\begin{tabular}{|c|c|c|c|c|c|c|c|}
\hline MG KR815 & C42 H62 N5 O9 & 781.4620 & $\begin{array}{l}\text { Cl- } N \mathrm{Me}- \\
\text { Ahda }\end{array}$ & & & & \\
\hline MG KR835 & $\begin{array}{l}\text { C41 H59 Cl2 N5 } \\
\text { O9 }\end{array}$ & 836.3763 & $\begin{array}{l}\mathrm{Cl} 2-N \mathrm{Me}- \\
\text { Ahda }\end{array}$ & Tyr & Me Leu & Pro & Tyr \\
\hline MG SD755 & C40 H61 N5 O9 & 755.4464 & MAhda & Val & Me Ile & Me Tyr & Tyr \\
\hline Microginin neu & & & & & & & \\
\hline $\begin{array}{l}\text { Microginine } \\
\text { microginine }\end{array}$ & C37 H55 N5 O9 & 714.4073 & Ahda & Ala & Val & Me Tyr & Tyr \\
\hline Microginine 711711 & C37 H53 N5 O9 & 712.3916 & An & Val & $\begin{array}{l}\text { Me Leu/Me } \\
\text { Ile }\end{array}$ & Tyr & Tyr \\
\hline Microginine 757 & C38 H59 N5 O10 & 745.4256 & Ahda & Thr & Me Leu & Tyr & Tyr \\
\hline Microginine 764764 & C40 H56 N6 O9 & 765.4182 & Me Ahda & Thr & Pro & Tyr & Trp \\
\hline Microginine 767767 & C41 H61 N5 O9 & 768.4542 & Me Ahda & Tyr & Me Ile & Pro & Tyr \\
\hline $\begin{array}{l}\text { Microginine GH787 } \\
\text { GH787 }\end{array}$ & $\begin{array}{l}\text { C40 H58 Cl N5 } \\
\text { O9 }\end{array}$ & 788.3996 & Cl Ahda & Tyr & Me Ile & Pro & Tyr \\
\hline $\begin{array}{l}\text { Microguanidine } \\
\text { KT636 }\end{array}$ & $\begin{array}{l}\text { C19 H32 N4 O14 } \\
\text { S }\end{array}$ & 572.1630 & & & & & \\
\hline Cyanostatin A & C38 H57 N5 O9 & 728.4229 & Ahda & Ala & Val & Me Tyr & Hty \\
\hline Cyanostatin B & C40 H59 N5 O9 & 754.4386 & Ahda & Tyr & Me Ile & Me Pro & Tyr \\
\hline Nostoginin BN578 & C30 H51 N4 O7 & 580.3831 & & & & & \\
\hline Nostoginin BN741 & C39 H59 N5 O9 & 742.4286 & & & & & \\
\hline Oscallaginin $\mathrm{A}$ & $\begin{array}{l}\mathrm{C} 29 \mathrm{H} 47 \mathrm{Cl} \mathrm{N} 4 \\
\mathrm{O} 8\end{array}$ & 615.3155 & Cl Ahda & Ser & Me Val & HTyr & $\mathrm{N} / \mathrm{A}$ \\
\hline Oscallaginin B & $\mathrm{C} 29 \mathrm{H} 48 \mathrm{~N} 4 \mathrm{O} 8$ & 581.3545 & Ahda & Ser & Me Val & HTyr & N/A \\
\hline
\end{tabular}

Figure S1: Standard curve for quantitative analysis of MC-LR in toxigenic Microcystis strain extracts. 


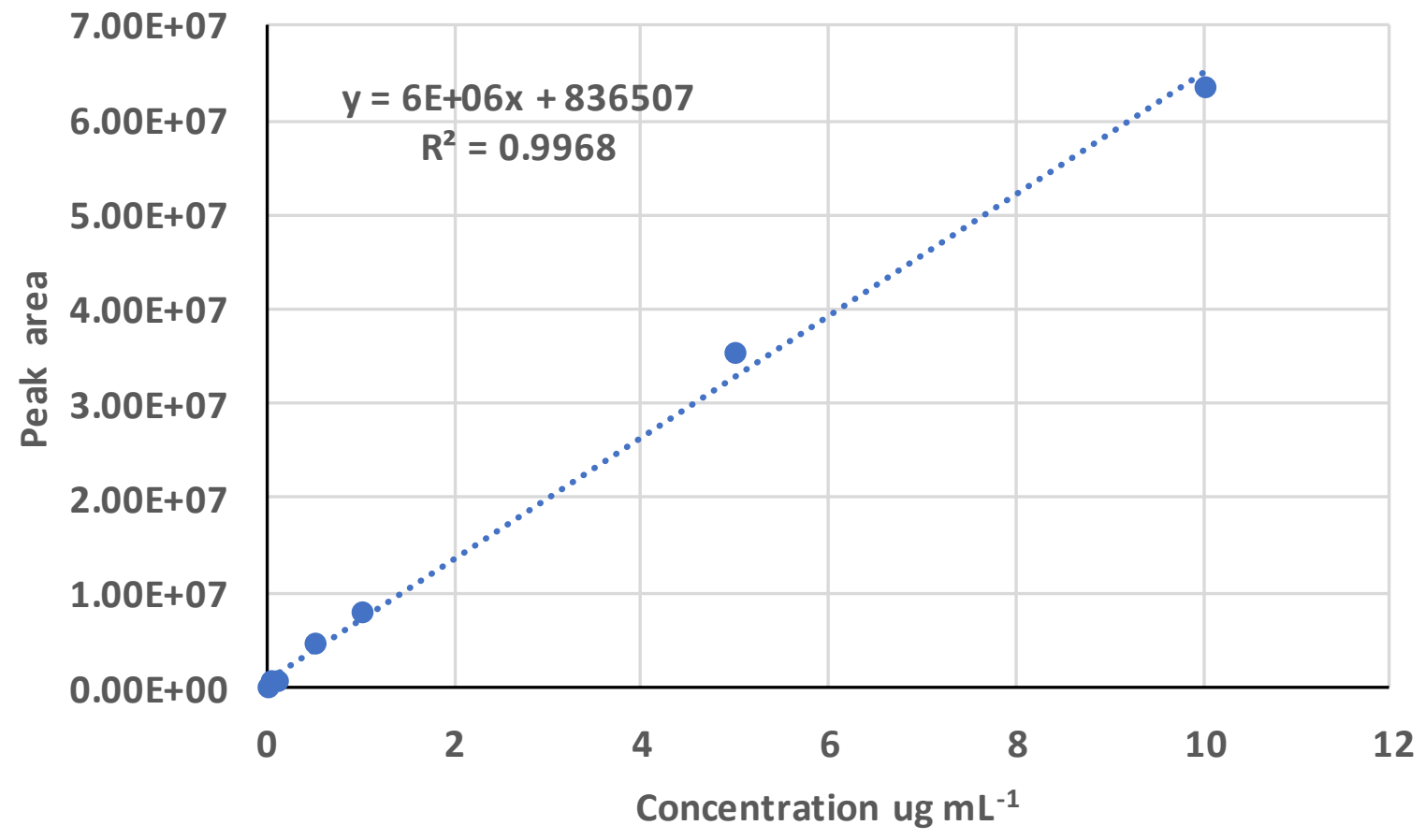

